\title{
Pd-Catalyzed Alkenyl Thioether Synthesis from Thioesters and $N$-Tosylhydrazones
}

Kota Ishitobi, Kei Muto, and Junichiro Yamaguchi*

Department of Applied Chemistry, Waseda University, 3-4-1 Ohkubo, Shinjuku, Tokyo 169-8555, Japan

E-mail: junyamaguchi@waseda.jp

\section{Table of Contents}

1. General

S2

2. Synthesis of Thioesters 1

S3-S4

3. Synthesis of $N$-Tosylhydrazone 2

S5-S11

4. Pd-Catalyzed Alkenyl Sulfide Synthesis

S12-S30

5. One-Pot Operation

6. Detection of Benzaldehyde using $\mathbf{1 K}$

S31

7 Derivatization of Product

8. Effect of Parameter

9. Reference

10. ${ }^{1} \mathrm{H}$ and ${ }^{13} \mathrm{C}$ NMR Spectra 


\section{General}

Unless otherwise noted, all reactants or reagents including dry solvents were obtained from commercial suppliers and used as received. $\mathrm{Pd}(\mathrm{OAc})_{2}$ was obtained from FUJIFILM Wako Pure Chemical Corporation. $\mathrm{P}^{n} \mathrm{Bu}_{3}$ was obtained from KANTO Chemical. $\mathrm{LiO}^{t} \mathrm{Bu}$ was obtained from Acros Organics. $S$-phenyl benzothioate $(\mathbf{1 A}),{ }^{[1]} S$-( $p$-tolyl) benzothioate $(\mathbf{1 E}),{ }^{[2]} S$-(o-tolyl) benzothioate (1F), ${ }^{[3]} S$-(3-methoxyphenyl) benzothioate $(\mathbf{1 G}),{ }^{[4]} S$-(4-methoxyphenyl) benzothioate $(\mathbf{1 H}){ }^{[1]}$ $S$-(naphthalen-2-yl) benzothioate (1I) ${ }^{[5]} S$-(4-bromophenyl) benzothioate (1J) ${ }^{[2]} S$-(4-chlorophenyl) benzothioate (1K) ${ }^{[6]} S$-(4-fluorophenyl) benzothioate (1L) ${ }^{[1]}$ methyl 4-(benzoylthio)benzoate (1M) ${ }^{[7]}$ $S$-(4-(trifluoromethyl)phenyl) benzothioate $\quad(\mathbf{1 P}),{ }^{[1]} \quad S$-(pyridin-4-yl) benzothioate $\quad(\mathbf{1 Q})^{[1]}$, (E)-4-methyl- $N^{\prime}$-(1-phenylpropylidene)benzenesulfonohydrazide $\quad$ (2a) ${ }^{[8]}$ (E)-4-methyl- $N$ '-(1-phenylbutylidene)benzenesulfonohydrazide $\quad$ (2c) ${ }^{[8]}$ (E)-4-methyl- $N$ '-(3-methyl-1-phenylbutylidene)benzenesulfonohydrazide $\quad$ (2d) ${ }^{[9]}$ (E)-4-methyl- $N$ '-(2-methyl-1-phenylpropylidene)benzenesulfonohydrazide $\quad$ (2e), ${ }^{[8]}$ (E)- $N^{\prime}$-(cyclohexyl(phenyl)methylene)-4-methylbenzenesulfonohydrazide $\quad$ (2f) ${ }^{[8]}$ (E)- $N^{\prime}$-(3,4-dihydronaphthalen-1(2H)-ylidene)-4-methylbenzenesulfonohydrazide $\quad(\mathbf{2 g}),{ }^{[8]}$ were synthesized according to procedures and the spectra matched with those of compounds reported in the literature. Unless otherwise noted, all reactions were performed with dry solvents under an atmosphere of $\mathrm{N}_{2}$ in dried glassware using standard vacuum-line techniques. All alkenyl sulfide synthesis reactions were performed in 20-mL glass vessel tubes equipped with J. Young ${ }^{\circledR}$ O-ring tap and heated (IKA Plate RCT digital) in an oil-bath or a 9-well aluminum reaction block (IKA H 135.103 Block 9 x 16 ml) unless otherwise noted. All work-up and purification procedures were carried out with reagent-grade solvents in air.

Analytical thin-layer chromatography (TLC) was performed using Silicagel 70 TLC Plate-Wako $(0.25 \mathrm{~mm})$. The developed chromatogram was analyzed by UV lamp $(254 \mathrm{~nm})$. Flash column chromatography was performed with Biotage Isolera ${ }^{\circledR}$ equipped with Biotage SNAP Cartridge KP-Sil columns. Preparative thin-layer chromatography (PTLC) was performed using Wakogel B5-F silica coated plates $(0.75 \mathrm{~mm})$ prepared in our laboratory. High-resolution mass spectra were conducted on Thermo Fisher Scientific ExactivePlus ${ }^{\circledR}$ (ESI and DART). Nuclear magnetic resonance (NMR) spectra were recorded on a JEOL JNM-ECS-400 $\left({ }^{1} \mathrm{H} 400 \mathrm{MHz},{ }^{13} \mathrm{C} 101 \mathrm{MHz}\right)$. Chemical shifts for ${ }^{1} \mathrm{H}$ NMR are expressed in parts per million $(\mathrm{ppm})$ relative to tetramethylsilane $(\delta 0.00 \mathrm{ppm})$ and DMSO $(\delta 2.50$ ppm). Chemical shifts for ${ }^{13} \mathrm{C}$ NMR are expressed in ppm relative to $\mathrm{CDCl}_{3}(\delta 77.0 \mathrm{ppm})$ and DMSO-d $_{6}(\delta 39.5 \mathrm{ppm})$. Data are reported as follows: chemical shift, multiplicity $(\mathrm{s}=$ singlet, brs $=$ broad singlet, $\mathrm{d}=$ doublet, $\mathrm{dd}=$ doublet of doublets, $\mathrm{ddd}=$ doublet of doublets of doublets, $\mathrm{t}=$ triplet, $\mathrm{td}=$ triplet of doublets, $\mathrm{q}=$ quartet, $\mathrm{m}=$ multiplet $)$, coupling constant $(\mathrm{Hz})$, and integration . 


\section{Synthesis of Thioesters 1}

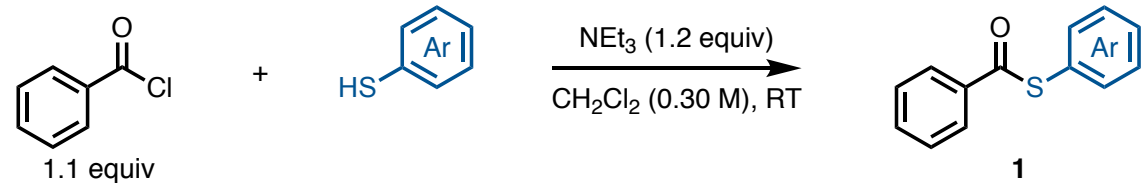

To a solution of the benzoyl chloride (1.1 equiv) in $\mathrm{CH}_{2} \mathrm{Cl}_{2}(0.30 \mathrm{M})$ was added thiols (1.0 equiv). Triethylamine (Et $3 \mathrm{~N}$ : 1.2 equiv) was slowly added to the mixture at $0{ }^{\circ} \mathrm{C}$. After stirring the mixture for several hours with monitoring the reaction progress by TLC. After the completion of reaction, the mixture was added water and extracted three times with $\mathrm{CH}_{2} \mathrm{Cl}_{2}$. The organic layer was dried over $\mathrm{Na}_{2} \mathrm{SO}_{4}$, filtrated, and then concentrated in vacuo. The residue was purified by Isolera ${ }^{\circledR}$ (hexane/EtOAc) and then recrystallization to afford the corresponding thioester $\mathbf{1}$.<smiles>CCc1ccc(SC(=O)c2ccccc2)cc1</smiles>

\section{$S$-(4-Ethylphenyl) benzothioate (1B)}

Purification by Isolera ${ }^{\circledR}$ (hexane/EtOAc $=99: 1$ to $9: 1$ ) and then recrystallization (hexane) afforded 1B as a white solid (913 mg, $5.00 \mathrm{mmol} \mathrm{scale,} 76 \%$ yield). ${ }^{1} \mathrm{H}$ NMR $\left(400 \mathrm{MHz}, \mathrm{CDCl}_{3}\right) \delta 8.03(\mathrm{~d}, J=6.8$ $\mathrm{Hz}, 2 \mathrm{H}), 7.61(\mathrm{t}, J=7.2 \mathrm{~Hz}, 1 \mathrm{H}), 7.49(\mathrm{dd}, J=7.2,6.8 \mathrm{~Hz}, 2 \mathrm{H}), 7.43(\mathrm{~d}, J=8.0 \mathrm{~Hz}, 2 \mathrm{H}), 7.30(\mathrm{~d}, J=$ $8.0 \mathrm{~Hz}, 2 \mathrm{H}), 2.71(\mathrm{q}, J=8.0 \mathrm{~Hz}, 2 \mathrm{H}), 1.27(\mathrm{t}, J=8.0 \mathrm{~Hz}, 3 \mathrm{H}) ;{ }^{13} \mathrm{C} \mathrm{NMR}\left(101 \mathrm{MHz}, \mathrm{CDCl}_{3}\right) \delta 190.6$, 146.0, 136.7, 135.0, 133.6, 128.9, 128.7, 127.5, 123.9, 28.7, 15.3; HRMS (DART) $\mathrm{m} / z$ calcd for $\mathrm{C}_{15} \mathrm{H}_{15} \mathrm{OS}[\mathrm{M}+\mathrm{H}]^{+}: 243.0838$ found 243.0836 .

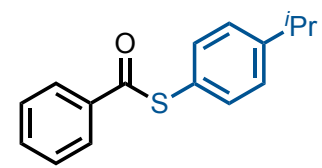

\section{$S$-(4-Isopropylphenyl) benzothioate (1C)}

Purification by Isolera ${ }^{\circledR}$ (hexane/EtOAc $=99: 1$ to $9: 1$ ) and then recrystallization (hexane) afforded 1C as a white solid (1.09 g, $5.00 \mathrm{mmol}$ scale, $86 \%$ yield). ${ }^{1} \mathrm{H}$ NMR $\left(400 \mathrm{MHz}, \mathrm{CDCl}_{3}\right) \delta 8.03(\mathrm{~d}, J=8.4$ $\mathrm{Hz}, 2 \mathrm{H}), 7.61(\mathrm{t}, J=8.0 \mathrm{~Hz}, 1 \mathrm{H}), 7.49(\mathrm{dd}, J=8.4,8.0 \mathrm{~Hz}, 2 \mathrm{H}), 7.43(\mathrm{~d}, J=8.0 \mathrm{~Hz}, 2 \mathrm{H}), 7.32(\mathrm{~d}, J=$ $8.0 \mathrm{~Hz}, 2 \mathrm{H}), 3.01-2.91(\mathrm{~m}, 1 \mathrm{H}), 1.28(\mathrm{~d}, J=6.8 \mathrm{~Hz}, 6 \mathrm{H}) ;{ }^{13} \mathrm{C} \mathrm{NMR}\left(101 \mathrm{MHz}, \mathrm{CDCl}_{3}\right) \delta 190.6,150.5$, 136.7, 135.0, 133.6, 128.7, 127.5, 127.4, 124.0, 34.0, 23.8; HRMS (DART) $\mathrm{m} / z$ calcd for $\mathrm{C}_{16} \mathrm{H}_{17} \mathrm{OS}$ $[\mathrm{M}+\mathrm{H}]^{+}: 257.0995$ found 257.0990 . 
<smiles>O=C(Sc1ccc(Br)cc1)c1ccccc1</smiles>

\section{$S$-(4-(tert-Butyl)phenyl) benzothioate (1D)}

Purification by Isolera ${ }^{\circledR}$ (hexane/EtOAc $=99: 1$ to 9:1) and then recrystallization (hexane) afforded 1D as a white solid (1.27 g, $5.00 \mathrm{mmol}$ scale, $95 \%$ yield). ${ }^{1} \mathrm{H}$ NMR (400 MHz, $\left.\mathrm{CDCl}_{3}\right) \delta 8.03(\mathrm{~d}, J=8.4$ $\mathrm{Hz}, 2 \mathrm{H}), 7.61(\mathrm{t}, J=7.6 \mathrm{~Hz}, 1 \mathrm{H}), 7.52-7.42(\mathrm{~m}, 6 \mathrm{H}), 1.35(\mathrm{~s}, 9 \mathrm{H}) ;{ }^{13} \mathrm{C} \mathrm{NMR}\left(101 \mathrm{MHz}, \mathrm{CDCl}_{3}\right) \delta$ 190.6, 152.7, 136.7, 134.7, 133.6, 128.7, 127.4, 126.4, 123.8, 34.8, 31.2; HRMS (DART) $\mathrm{m} / z$ calcd for $\mathrm{C}_{17} \mathrm{H}_{19} \mathrm{OS}[\mathrm{M}+\mathrm{H}]^{+}: 271.1151$ found 271.1147.

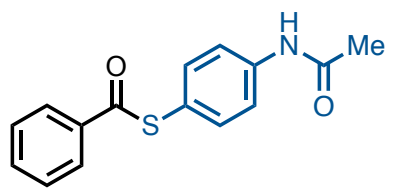

\section{$S$-(4-Acetamidophenyl) benzothioate (1N)}

Purification by Isolera ${ }^{\circledR}\left(\mathrm{CHCl}_{3} / \mathrm{MeOH}=99: 1\right.$ to $\left.9: 1\right)$ and then recrystallization (hexane/ $\left.\mathrm{CH}_{2} \mathrm{Cl}_{2}\right)$ afforded $1 \mathrm{~N}$ as a white solid (913 mg, $5.0 \mathrm{mmol}$ scale, 67\% yield). ${ }^{1} \mathrm{H}$ NMR (400 MHz, DMSO-d 6 ) $\delta$ 10.19 (s, 1H), 7.97 (d, $J=7.2 \mathrm{~Hz}, 2 \mathrm{H}), 7.76-7.69$ (m, 3H), 7.59 (dd, $J=7.6,7.2 \mathrm{~Hz}, 2 \mathrm{H}), 7.43$ (d, $J=$ $8.8 \mathrm{~Hz}, 2 \mathrm{H}), 2.08(\mathrm{~s}, 3 \mathrm{H}) ;{ }^{13} \mathrm{C}$ NMR $\left(101 \mathrm{MHz}, \mathrm{DMSO}_{-} \mathrm{d}_{6}\right) \delta 189.8,168.8,140.8,135.9,135.8,134.3$, 129.3, 127.1, 119.7, 24.2 (one peak is missing due to overlapping); HRMS (DART) $\mathrm{m} / \mathrm{z}$ calcd for $\mathrm{C}_{15} \mathrm{H}_{14} \mathrm{NO}_{2} \mathrm{~S}[\mathrm{M}+\mathrm{H}]^{+}: 272.0740$ found 272.0734.

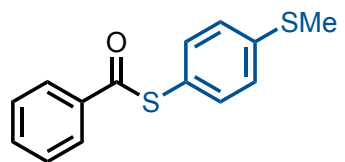

\section{$S$-(4-(Methylthio)phenyl) benzothioate (10)}

Purification by Isolera ${ }^{\circledR}$ (hexane/EtOAc $=99: 1$ to 9:1) and then recrystallization (hexane) afforded 10 as a white solid (711 mg, $5.0 \mathrm{mmol}$ scale, $55 \%$ yield). ${ }^{1} \mathrm{H}$ NMR $\left(400 \mathrm{MHz}, \mathrm{CDCl}_{3}\right) \delta 8.02(\mathrm{~d}, J=8.0$ $\mathrm{Hz}, 2 \mathrm{H}), 7.61(\mathrm{t}, J=7.2 \mathrm{~Hz}, 1 \mathrm{H}), 7.49$ (dd, $J=8.0,7.2 \mathrm{~Hz}, 2 \mathrm{H}), 7.41$ (d, $J=8.4 \mathrm{~Hz}, 2 \mathrm{H}), 7.31$ (d, $J=$ $8.4 \mathrm{~Hz}, 2 \mathrm{H}), 2.52(\mathrm{~s}, 3 \mathrm{H}) ;{ }^{13} \mathrm{C} \mathrm{NMR}\left(101 \mathrm{MHz}, \mathrm{CDCl}_{3}\right) \delta 190.4,141.2,136.5,135.3,133.7,128.7$, 127.5, 126.6, 123.0, 15.3; HRMS (DART) $\mathrm{m} / z$ calcd for $\mathrm{C}_{14} \mathrm{H}_{13} \mathrm{OS}_{2}[\mathrm{M}+\mathrm{H}]^{+}: 261.0402$ found 261.0400 . 


\section{Synthesis of $N$-Tosylhydrazone 2}

\section{3-1. Synthesis of $N$-Tosylhydrazone 2 from ketone}

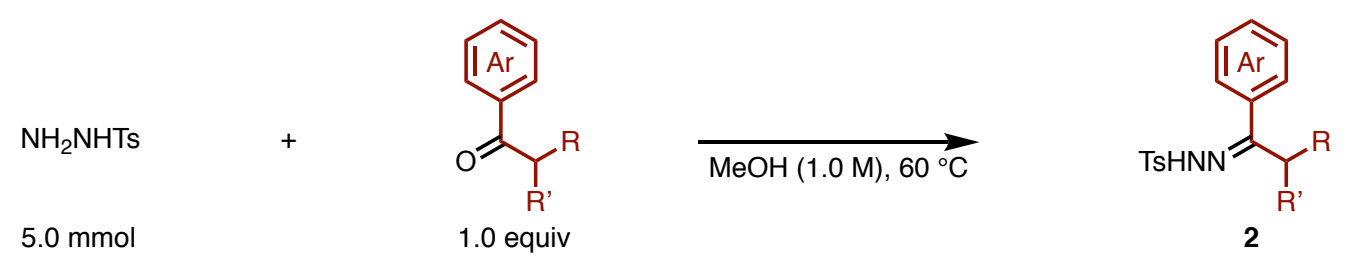

$N$-Tosylhydrazones was prepared according to literature procedure. ${ }^{[10]}$ A solution of $\mathrm{TsNHNH}_{2}$ (931 mg, $5.0 \mathrm{mmol}, 1$ equiv) in methanol $(5.0 \mathrm{~mL})$ was stirred and heated to $60{ }^{\circ} \mathrm{C}$ until the $\mathrm{TsNHNH}_{2}$ was completely dissolved. To this solution, aryl ketones $(5.0 \mathrm{mmol}, 1.0$ equiv) was added to the mixture slowly via syringe. After approximately 5-30 min, $N$-tosylhydrazones was precipitated. The precipitate was collected by filtration, and then the solid was washed by hexane. The precipitate was purified by recrystallization to afford the corresponding $N$-tosylhydrazones $\mathbf{2}$.

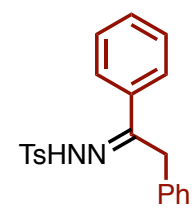

\section{$N^{\prime}$-(1,2-Diphenylethylidene)-4-methylbenzenesulfonohydrazide (2b)}

Purification by recrystallization (hexane $/ \mathrm{CH}_{2} \mathrm{Cl}_{2}$ ) afforded $\mathbf{2 b}$ as a white solid (1.37 g, $5.00 \mathrm{mmol}$ scale, $76 \%$ yield). ${ }^{1} \mathrm{H}$ NMR (400 MHz, $\left.\mathrm{CDCl}_{3}\right) \delta$ 7.71-7.66 (m, 4H), 7.48 (s, 1H), 7.38-7.31 (m, 3H), 7.29$7.18(\mathrm{~m}, 5 \mathrm{H}), 6.95(\mathrm{~d}, J=6.4 \mathrm{~Hz}, 2 \mathrm{H}), 4.01(\mathrm{~s}, 2 \mathrm{H}), 2.42(\mathrm{~s}, 3 \mathrm{H}) ;{ }^{13} \mathrm{C} \mathrm{NMR}\left(101 \mathrm{MHz}, \mathrm{CDCl}_{3}\right) \delta$ 153.3, 144.1, 136.9, 135.1, 133.3, 129.7, 129.5, 129.4, 128.5, 127.9, 127.6, 127.4, 126.5, 33.3, 21.6; HRMS (DART) $m / z$ calcd for $\mathrm{C}_{21} \mathrm{H}_{21} \mathrm{~N}_{2} \mathrm{O}_{2} \mathrm{~S}[\mathrm{M}+\mathrm{H}]^{+}: 365.1318$ found 365.1313.

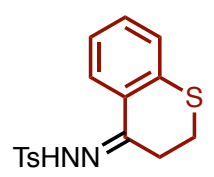

\section{4-Methyl- $N$ '-(thiochroman-4-ylidene)benzenesulfonohydrazide (2h)}

Purification by recrystallization (hexane $/ \mathrm{CH}_{2} \mathrm{Cl}_{2}$ ) afforded $\mathbf{2 h}$ as a white solid $(924 \mathrm{mg}, 3.95 \mathrm{mmol}$ scale, 71\% yield). ${ }^{1} \mathrm{H}$ NMR (400 MHz, $\left.\mathrm{CDCl}_{3}\right) \delta 8.01(\mathrm{~d}, J=8.0 \mathrm{~Hz}, 1 \mathrm{H}), 7.91(\mathrm{~d}, J=8.0 \mathrm{~Hz}, 2 \mathrm{H})$, $7.48(\mathrm{~s}, 1 \mathrm{H}), 7.33(\mathrm{~d}, J=8.0 \mathrm{~Hz}, 2 \mathrm{H}), 7.21-7.18(\mathrm{~m}, 2 \mathrm{H}), 7.16-7.10(\mathrm{~m}, 1 \mathrm{H}), 2.97(\mathrm{dd}, J=6.4,6.0 \mathrm{~Hz}$, 2H), $2.81(\mathrm{dd}, J=6.4,6.0 \mathrm{~Hz}, 2 \mathrm{H}), 2.42(\mathrm{~s}, 3 \mathrm{H}) ;{ }^{13} \mathrm{C} \mathrm{NMR}\left(101 \mathrm{MHz}, \mathrm{CDCl}_{3}\right) \delta 150.0,144.4,136.2$, 135.1, 130.7, 129.7, 129.5, 128.2, 128.1, 126.9, 125.6, 27.8, 25.7, 21.6; HRMS (DART) $\mathrm{m} / z$ calcd for $\mathrm{C}_{16} \mathrm{H}_{17} \mathrm{~N}_{2} \mathrm{O}_{2} \mathrm{~S}_{2}[\mathrm{M}+\mathrm{H}]^{+}: 333.0726$ found 333.0720 . 


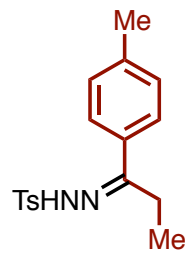

\section{4-Methyl- $N^{\prime}$-(1-(p-tolyl)propylidene)benzenesulfonohydrazide (2i)}

Purification by recrystallization (hexane/ $\mathrm{CH}_{2} \mathrm{Cl}_{2}$ ) afforded $\mathbf{2 i}$ as a white solid $(797 \mathrm{mg}, 5.00 \mathrm{mmol}$ scale, $84 \%$ yield). ${ }^{1} \mathrm{H}$ NMR (400 MHz, $\left.\mathrm{CDCl}_{3}\right) \delta 8.04-7.81(\mathrm{~m}, 3 \mathrm{H}), 7.54(\mathrm{~d}, J=8.8 \mathrm{~Hz}, 2 \mathrm{H}), 7.31(\mathrm{~d}$, $J=8.8 \mathrm{~Hz}, 2 \mathrm{H}), 7.15(\mathrm{~d}, J=8.0 \mathrm{~Hz}, 2 \mathrm{H}), 2.57$ (q, $J=8.0 \mathrm{~Hz}, 2 \mathrm{H}), 2.41(\mathrm{~s}, 3 \mathrm{H}), 2.35$ (s, 3H), 1.08 (t, $J$ $=8.0 \mathrm{~Hz}, 3 \mathrm{H}) ;{ }^{13} \mathrm{C} \mathrm{NMR}\left(101 \mathrm{MHz}, \mathrm{CDCl}_{3}\right) \delta 157.0,144.0,139.8,135.4,133.3,129.6,129.1,128.0$, 126.2, 21.6, 21.2, 19.9, 10.3; HRMS (DART) $\mathrm{m} / z$ calcd for $\mathrm{C}_{17} \mathrm{H}_{21} \mathrm{~N}_{2} \mathrm{O}_{2} \mathrm{~S}[\mathrm{M}+\mathrm{H}]^{+}: 317.1318$ found 317.1312 .<smiles></smiles>

\section{$N^{\prime}$-(1-(4-(Tert-butyl)phenyl)propylidene)-4-methylbenzenesulfonohydrazide (2j)}

Purification by recrystallization (hexane $/ \mathrm{CH}_{2} \mathrm{Cl}_{2}$ ) afforded $2 \mathbf{j}$ as a white solid $(1.50 \mathrm{~g}, 5.00 \mathrm{mmol}$ scale, 84\% yield). ${ }^{1} \mathrm{H}$ NMR $\left(400 \mathrm{MHz}, \mathrm{CDCl}_{3}\right) \delta 7.90$ (d, $\left.J=8.0 \mathrm{~Hz}, 2 \mathrm{H}\right), 7.59$ (d, $\left.J=8.8 \mathrm{~Hz}, 2 \mathrm{H}\right), 7.53-$ 7.50 (brs, $1 \mathrm{H}), 7.37$ (d, $J=8.8 \mathrm{~Hz}, 2 \mathrm{H}), 7.31$ (d, $J=8.0 \mathrm{~Hz}, 2 \mathrm{H}), 2.56$ (q, $J=8.0 \mathrm{~Hz}, 2 \mathrm{H}), 2.41$ (s, 3H), $1.31(\mathrm{~s}, 9 \mathrm{H}), 1.09(\mathrm{t}, J=8.0 \mathrm{~Hz}, 3 \mathrm{H}) ;{ }^{13} \mathrm{C} \mathrm{NMR}\left(101 \mathrm{MHz}, \mathrm{CDCl}_{3}\right) \delta 157.0,152.7,144.0,135.4,133.3$, 129.5, 128.0, 126.0, 125.3, 34.6, 31.1, 21.5, 20.0, 10.3; HRMS (DART) $m / z$ calcd for $\mathrm{C}_{20} \mathrm{H}_{27} \mathrm{~N}_{2} \mathrm{O}_{2} \mathrm{~S}$ $[\mathrm{M}+\mathrm{H}]^{+}: 359.1788$ found 359.1779 .<smiles>N#CC(=N)c1ccc(-c2ccccc2)cc1</smiles>

\section{$N^{\prime}$-(1-([1,1'-Biphenyl]-4-yl)propylidene)-4-methylbenzenesulfonohydrazide (2k)}

Purification by recrystallization (hexane/ $\mathrm{CH}_{2} \mathrm{Cl}_{2}$ ) afforded $\mathbf{2 k}$ as a white solid (1.46 g, $5.00 \mathrm{mmol}$ scale, $77 \%$ yield). ${ }^{1} \mathrm{H}$ NMR $\left(400 \mathrm{MHz}, \mathrm{CDCl}_{3}\right) \delta 8.12-8.02$ (brs, $\left.1 \mathrm{H}\right), 7.94(\mathrm{t}, J=8.0 \mathrm{~Hz}, 2 \mathrm{H}), 7.73$ (d, $J=$ $8.8 \mathrm{~Hz}, 2 \mathrm{H}), 7.61-7.57(\mathrm{~m}, 4 \mathrm{H}), 7.45(\mathrm{t}, J=8.0 \mathrm{~Hz}, 2 \mathrm{H}), 7.39-7.32(\mathrm{~m}, 3 \mathrm{H}), 2.67-2.58(\mathrm{~m}, 2 \mathrm{H}), 2.42$ (s, 3H), $1.13(\mathrm{t}, J=8.0 \mathrm{~Hz}, 3 \mathrm{H}) ;{ }^{13} \mathrm{C}$ NMR $\left(101 \mathrm{MHz}, \mathrm{CDCl}_{3}\right) \delta 156.5,144.2,142.3,140.3,135.4$, 135.0, 129.6, 128.8, 128.1, 127.7, 127.1, 127.0, 126.8, 21.6, 19.9, 10.3; HRMS (ESI) $\mathrm{m} / \mathrm{z}$ calcd for $\mathrm{C}_{22} \mathrm{H}_{23} \mathrm{~N}_{2} \mathrm{O}_{2} \mathrm{~S}[\mathrm{M}+\mathrm{H}]^{+}: 379.1475$ found 379.1470 . 


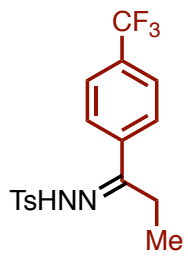

\section{4-Methyl- $N$ '-(1-(4-(trifluoromethyl)phenyl)propylidene)benzenesulfonohydrazide (2n)}

Purification by recrystallization (hexane $/ \mathrm{CH}_{2} \mathrm{Cl}_{2}$ ) afforded $\mathbf{2 n}$ as a white solid $(647 \mathrm{mg}, 5.00 \mathrm{mmol}$ scale, 58\% yield). ${ }^{1} \mathrm{H}$ NMR (400 MHz, $\left.\mathrm{CDCl}_{3}\right) \delta 7.90(\mathrm{~d}, J=8.8 \mathrm{~Hz}, 2 \mathrm{H}), 7.75(\mathrm{~d}, J=8.4 \mathrm{~Hz}, 2 \mathrm{H})$, 7.69 (s, 1H), 7.61 (d, $J=8.4 \mathrm{~Hz}, 2 \mathrm{H}), 7.33$ (d, $J=8.8 \mathrm{~Hz}, 2 \mathrm{H}), 2.59$ (q, $J=8.0 \mathrm{~Hz}, 2 \mathrm{H}), 2.42$ (s, 3H), $1.11(\mathrm{t}, J=8.0 \mathrm{~Hz}, 3 \mathrm{H}) ;{ }^{13} \mathrm{C}$ NMR $\left(101 \mathrm{MHz}, \mathrm{CDCl}_{3}\right) \delta 155.2,144.4,139.5,135.2,131.2\left(\mathrm{q}, J_{\mathrm{C}-\mathrm{F}}=\right.$ $33.8 \mathrm{~Hz}), 129.7,128.0,126.6,125.4\left(\mathrm{q}, J_{\mathrm{C}-\mathrm{F}}=2.9 \mathrm{~Hz}\right), 123.9$ (q, $\left.J_{\mathrm{C}-\mathrm{F}}=268.0 \mathrm{~Hz}\right), 21.6,20.0,10.1$; HRMS (DART) $m / z$ calcd for $\mathrm{C}_{17} \mathrm{H}_{18} \mathrm{~F}_{3} \mathrm{~N}_{2} \mathrm{O}_{2} \mathrm{~S}[\mathrm{M}+\mathrm{H}]^{+}: 371.1036$ found 371.1027 .<smiles>N=C(CN=[18O])c1ccc(Br)cc1</smiles>

\section{$N^{\prime}$-(1-(4-Bromophenyl)propylidene)-4-methylbenzenesulfonohydrazide (2o)}

Purification by recrystallization (hexane/ $\mathrm{CH}_{2} \mathrm{Cl}_{2}$ ) afforded $\mathbf{2 0}$ as a white solid (1.02 g, $3.00 \mathrm{mmol}$ scale, $89 \%$ yield). ${ }^{1} \mathrm{H}$ NMR (400 MHz, $\left.\mathrm{CDCl}_{3}\right) \delta 8.02-7.98$ (brs, $\left.1 \mathrm{H}\right), 7.90$ (d, $\left.J=8.4 \mathrm{~Hz}, 2 \mathrm{H}\right), 7.51$ (d, $J=$ $9.2 \mathrm{~Hz}, 2 \mathrm{H}), 7.46$ (d, $J=9.2 \mathrm{~Hz}, 2 \mathrm{H}), 7.32$ (d, $J=8.4 \mathrm{~Hz}, 2 \mathrm{H}), 2.56$ (q, $J=8.0 \mathrm{~Hz}, 2 \mathrm{H}), 2.42(\mathrm{~s}, 3 \mathrm{H})$, $1.08(\mathrm{t}, J=8.0 \mathrm{~Hz}, 3 \mathrm{H}) ;{ }^{13} \mathrm{C} \mathrm{NMR}\left(101 \mathrm{MHz}, \mathrm{CDCl}_{3}\right) \delta 155.6,144.3,135.2,135.0,131.6,129.7$, 128.0, 127.8, 123.9, 21.6, 19.8, 10.2; HRMS (DART) $\mathrm{m} / z$ calcd for $\mathrm{C}_{16} \mathrm{H}_{18} \mathrm{BrN}_{2} \mathrm{O}_{2} \mathrm{~S}[\mathrm{M}+\mathrm{H}]^{+}: 381.0267$ found 381.0258 .<smiles>N=C(CN=[W])c1ccc(Cl)cc1</smiles>

\section{$N^{\prime}$-(1-(4-Chlorophenyl)propylidene)-4-methylbenzenesulfonohydrazide (2p)}

Purification by recrystallization (hexane/ $\mathrm{CH}_{2} \mathrm{Cl}_{2}$ ) afforded $\mathbf{2 p}$ as a white solid $(531 \mathrm{mg}, 2.63 \mathrm{mmol}$ scale, $60 \%$ yield). ${ }^{1} \mathrm{H}$ NMR (400 MHz, $\left.\mathrm{CDCl}_{3}\right) \delta 8.18-8.09$ (brs, $\left.1 \mathrm{H}\right), 7.91(\mathrm{~d}, J=8.4 \mathrm{~Hz}, 2 \mathrm{H}), 7.57$ (d, $J=9.2 \mathrm{~Hz}, 2 \mathrm{H}), 7.34-7.29(\mathrm{~m}, 4 \mathrm{H}), 2.57$ (q, $J=8.0 \mathrm{~Hz}, 2 \mathrm{H}), 2.42(\mathrm{~s}, 3 \mathrm{H}), 1.08(\mathrm{t}, J=8.0 \mathrm{~Hz}, 3 \mathrm{H}) ;{ }^{13} \mathrm{C}$ NMR $\left(101 \mathrm{MHz}, \mathrm{CDCl}_{3}\right) \delta 155.7,144.3,135.6,135.3,134.6,129.7,128.6,128.0,127.6,21.6,19.9$, 10.2; HRMS (DART) $m / z$ calcd for $\mathrm{C}_{16} \mathrm{H}_{18} \mathrm{ClN}_{2} \mathrm{O}_{2} \mathrm{~S}[\mathrm{M}+\mathrm{H}]^{+}: 337.0772$ found 337.0776. 
<smiles>CCC(=[NH2+])c1cccc(Cl)c1</smiles>

\section{$N^{\prime}$-(1-(3-Chlorophenyl)propylidene)-4-methylbenzenesulfonohydrazide (2q)}

Purification by recrystallization (hexane/ $\mathrm{CH}_{2} \mathrm{Cl}_{2}$ ) afforded $\mathbf{2 q}$ as a white solid (1.41 g, $5.00 \mathrm{mmol}$ scale, 84\% yield). ${ }^{1} \mathrm{H}$ NMR (400 MHz, $\left.\mathrm{CDCl}_{3}\right) \delta 7.90$ (d, $\left.J=8.4 \mathrm{~Hz}, 2 \mathrm{H}\right), 7.63(\mathrm{~s}, 1 \mathrm{H}), 7.61$ (t, $J=1.6 \mathrm{~Hz}$, $1 \mathrm{H}), 7.51(\mathrm{dt}, J=8.0,1.6 \mathrm{~Hz}, 1 \mathrm{H}), 7.36-7.31(\mathrm{~m}, 3 \mathrm{H}), 7.29(\mathrm{~d}, J=7.6 \mathrm{~Hz}, 1 \mathrm{H}), 2.54(\mathrm{q}, J=8.0 \mathrm{~Hz}$, 2H), $2.43(\mathrm{~s}, 3 \mathrm{H}), 1.09(\mathrm{t}, J=8.0 \mathrm{~Hz}, 3 \mathrm{H}) ;{ }^{13} \mathrm{C} \mathrm{NMR}\left(101 \mathrm{MHz}, \mathrm{CDCl}_{3}\right) \delta 155.2,144.4,137.9,135.1$, 134.5, 129.7, 129.6, 129.5, 128.0, 126.5, 124.4, 21.6, 19.9, 10.1; HRMS (DART) $\mathrm{m} / \mathrm{z}$ calcd for $\mathrm{C}_{16} \mathrm{H}_{18} \mathrm{ClN}_{2} \mathrm{O}_{2} \mathrm{~S}[\mathrm{M}+\mathrm{H}]^{+}: 337.0772$ found 337.0766.

\section{3-2. Synthesis of $N$-Tosylhydrazone 2 from carboxylic acid}

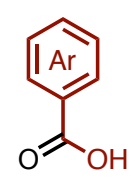

$5.0 \mathrm{mmol}$<smiles>CON(C)C(=O)c1ccccc1</smiles>
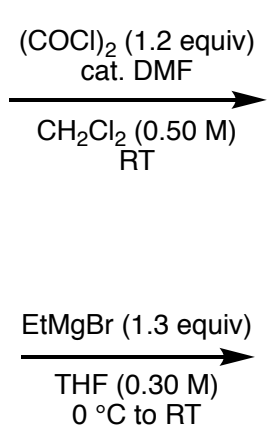<smiles>O=C(Cl)c1ccccc1</smiles><smiles>CCC(=O)c1ccccc1</smiles>
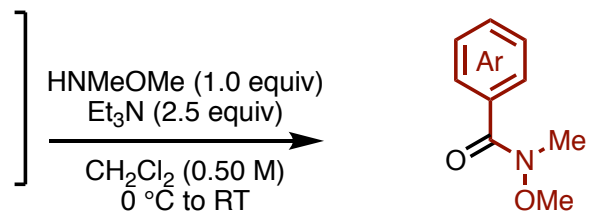

$\frac{\mathrm{NH}_{2} \mathrm{NHTs} \text { (1.0 equiv) }}{\mathrm{MeOH}(1.0 \mathrm{M}), 60^{\circ} \mathrm{C}}$

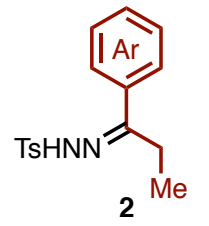

To a solution of the carboxylic acid $\left(5.0 \mathrm{mmol}, 1.0\right.$ equiv) in $\mathrm{CH}_{2} \mathrm{Cl}_{2}(0.50 \mathrm{M})$ were added oxalyl chloride $(515 \mu \mathrm{L}, 6.0 \mathrm{mmol}, 1.2$ equiv) and a portion of DMF. After stirring for $1.5 \mathrm{~h}$ at room temperature, $\mathrm{N}, \mathrm{O}$-dimethylhydroxylamine $(918 \mathrm{mg}, 5.0 \mathrm{mmol}, 1.0$ equiv) was added to the mixture. Triethylamine ( $\mathrm{Et}_{3} \mathrm{~N}: 1.74 \mathrm{~mL}, 12.5 \mathrm{mmol}, 2.5$ equiv) was slowly added to the mixture at $0{ }^{\circ} \mathrm{C}$. After stirring the mixture for several hours with monitoring the reaction progress by $\mathrm{TLC}, \mathrm{NaHCO}_{3}$ aq. was added to the resulting mixture to quench the reaction. The mixture was extracted three times with $\mathrm{CH}_{2} \mathrm{Cl}_{2}$. The combined organic layer was dried over $\mathrm{Na}_{2} \mathrm{SO}_{4}$, filtrated, and concentrated in vacuo. Purification by Isolera ${ }^{\circledR}$ afforded the corresponding Weinreb amide.

To a solution of the Weinreb amide in THF $(0.30 \mathrm{M})$ was slowly added ethylmagnesium bromide (EtMgBr: 1.0 equiv) at $0{ }^{\circ} \mathrm{C}$. After stirring the mixture for several hours with monitoring the reaction progress by $\mathrm{TLC}, \mathrm{NH}_{4} \mathrm{Cl}$ aq. was added to the resulting mixture to quench the reaction. The mixture was extracted three times with $\mathrm{CH}_{2} \mathrm{Cl}_{2}$. The combined organic layer was dried over $\mathrm{MgSO}_{4}$, filtrated, and concentrated in vacuo. Purification by Isolera ${ }^{\circledR}$ afforded the corresponding ketones.

A solution of $\mathrm{TsNHNH}_{2}\left(1.0\right.$ equiv) in methanol $(1.0 \mathrm{M})$ was stirred and heated to $60{ }^{\circ} \mathrm{C}$ until the $\mathrm{TsNHNH}_{2}$ was completely dissolved. The ketone was then added to the mixture slowly via syringe. After approximately 5-30 min, $N$-tosylhydrazones was precipitated. The precipitate was collected by 
filtration, and then the solid was washed by hexane. The precipitate was purified by recrystallization to afford the corresponding $N$-tosylhydrazones 2 .<smiles>CN(C)CCC(=O)c1ccc(N(C)C)cc1</smiles>

\section{$N^{\prime}$-(1-(4-(Dimethylamino)phenyl)propylidene)-4-methylbenzenesulfonohydrazide (2I)}

Purification by recrystallization (hexane/ $\mathrm{CH}_{2} \mathrm{Cl}_{2}$ ) afforded $\mathbf{2 l}$ as a white solid (708 $\mathrm{mg}, 41 \%$ yield over 3 steps). ${ }^{1} \mathrm{H}$ NMR (400 MHz, $\left.\mathrm{CDCl}_{3}\right) \delta 7.90$ (d, $\left.J=8.0 \mathrm{~Hz}, 2 \mathrm{H}\right), 7.57(\mathrm{~d}, J=9.2 \mathrm{~Hz}, 2 \mathrm{H}), 7.30$ (d, $J=$ $8.0 \mathrm{~Hz}, 2 \mathrm{H}), 6.64$ (d, $J=9.2 \mathrm{~Hz}, 2 \mathrm{H}), 2.99$ (s, 6H), 2.53 (q, $J=7.6 \mathrm{~Hz}, 2 \mathrm{H}), 2.40$ (s, 3H), 1.07 (t, $J=$ $7.6 \mathrm{~Hz}, 3 \mathrm{H}) ;{ }^{13} \mathrm{C}$ NMR $\left(101 \mathrm{MHz}, \mathrm{CDCl}_{3}\right) \delta 157.9,151.2,143.8,135.5,129.5,128.0,127.5,123.6$, 111.5, 40.2, 21.6, 19.6, 10.6; HRMS (DART) $m / z$ calcd for $\mathrm{C}_{18} \mathrm{H}_{24} \mathrm{~N}_{3} \mathrm{O}_{2} \mathrm{~S}[\mathrm{M}+\mathrm{H}]^{+}: 346.1584$ found 346.1576 .<smiles>N#Cc1ccc(C(=N)O[Na])cc1</smiles>

\section{$N^{\prime}$-(1-(4-Cyanophenyl)propylidene)-4-methylbenzenesulfonohydrazide (2m)}

Purification by recrystallization (hexane $/ \mathrm{CH}_{2} \mathrm{Cl}_{2}$ ) afforded $\mathbf{2 m}$ as a white solid (662 $\mathrm{mg}, 40 \%$ yield over 3 steps). ${ }^{1} \mathrm{H}$ NMR (400 MHz, $\left.\mathrm{CDCl}_{3}\right) \delta 7.89(\mathrm{~d}, J=8.0 \mathrm{~Hz}, 2 \mathrm{H}), 7.76-7.72(\mathrm{~m}, 3 \mathrm{H}), 7.64(\mathrm{~d}, J=$ $8.4 \mathrm{~Hz}, 2 \mathrm{H}), 7.34$ (d, $J=8.0 \mathrm{~Hz}, 2 \mathrm{H}), 2.57$ (q, $J=8.0 \mathrm{~Hz}, 2 \mathrm{H}), 2.43(\mathrm{~s}, 3 \mathrm{H}), 1.11$ (t, $J=8.0 \mathrm{~Hz}, 3 \mathrm{H})$; ${ }^{13} \mathrm{C}$ NMR $\left(101 \mathrm{MHz}, \mathrm{CDCl}_{3}\right) \delta 154.2,144.5,140.3,135.1,132.2,129.8,127.9,126.7,118.5,112.8$, 21.6, 19.8, 10.0; HRMS (DART) $\mathrm{m} / z$ calcd for $\mathrm{C}_{17} \mathrm{H}_{18} \mathrm{~N}_{3} \mathrm{O}_{2} \mathrm{~S}[\mathrm{M}+\mathrm{H}]^{+}: 328.1114$ found 328.1107.<smiles>CC(=N)c1ccc2ccccc2c1</smiles>

\section{4-Methyl- $N$ '-(1-(naphthalen-2-yl)propylidene)benzenesulfonohydrazide (2r)}

Purification by recrystallization (hexane $/ \mathrm{CH}_{2} \mathrm{Cl}_{2}$ ) afforded $\mathbf{2 r}$ as a white solid $(597.5 \mathrm{mg}, 34 \%$ yield over 3 steps). ${ }^{1} \mathrm{H}$ NMR (400 MHz, $\left.\mathrm{CDCl}_{3}\right) \delta$ 7.98-7.92 (m, 4H), 7.85-7.79 (m, 3H), $7.67(\mathrm{~s}, 1 \mathrm{H})$, $7.52-7.48$ (m, 2H), 7.33 (d, $J=8.8 \mathrm{~Hz}, 2 \mathrm{H}), 2.70$ (q, $J=8.0 \mathrm{~Hz}, 2 \mathrm{H}), 2.41$ (s, 3H), 1.17 (t, $J=8.0 \mathrm{~Hz}$, $3 \mathrm{H}) ;{ }^{13} \mathrm{C}$ NMR $\left(101 \mathrm{MHz}, \mathrm{CDCl}_{3}\right) \delta 156.8,144.2,135.4,133.8,133.5,132.9,129.7,128.5,128.0$, 
127.6, 126.9, 126.3, 126.1, 123.8, 21.6, 19.9, 10.5 (one peak is missing due to overlapping.); HRMS (DART) $m / z$ calcd for $\mathrm{C}_{20} \mathrm{H}_{21} \mathrm{~N}_{2} \mathrm{O}_{2} \mathrm{~S}[\mathrm{M}+\mathrm{H}]^{+}: 353.1318$ found 353.1311 .

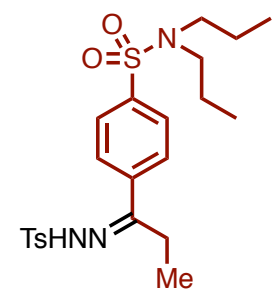

\section{$N, N$-Dipropyl-4-(1-(2-tosylhydrazineylidene)propyl)benzenesulfonamide (2s)}

Purification by recrystallization (hexane/ $\mathrm{CH}_{2} \mathrm{Cl}_{2}$ ) afforded $\mathbf{2 s}$ as a white solid ( $809 \mathrm{mg}$, 35\% yield over 3 steps). ${ }^{1} \mathrm{H}$ NMR (400 MHz, $\left.\mathrm{CDCl}_{3}\right) \delta 7.90$ (d, $\left.J=8.8 \mathrm{~Hz}, 2 \mathrm{H}\right), 7.79-7.72(\mathrm{~m}, 4 \mathrm{H}), 7.70(\mathrm{~s}, 1 \mathrm{H}), 7.35$ (d, $J=8.4 \mathrm{~Hz}, 2 \mathrm{H}), 3.10-3.05$ (m, 4H), 2.58 (q, $J=8.0 \mathrm{~Hz}, 2 \mathrm{H}), 2.44$ (s, 3H), 1.61-1.50 (m, 4H), 1.12 $(\mathrm{t}, J=8.0 \mathrm{~Hz}, 3 \mathrm{H}), 0.88(\mathrm{t}, J=8.0 \mathrm{~Hz}, 6 \mathrm{H}) ;{ }^{13} \mathrm{C} \mathrm{NMR}\left(101 \mathrm{MHz}, \mathrm{CDCl}_{3}\right) \delta 154.8,144.5,140.7,139.8$, 135.1, 129.7, 128.0, 127.1, 126.7, 50.0, 22.0, 21.6, 20.0, 11.2, 10.1; HRMS (DART) $\mathrm{m} / \mathrm{z}$ calcd for $\mathrm{C}_{22} \mathrm{H}_{32} \mathrm{~N}_{3} \mathrm{O}_{4} \mathrm{~S}_{2}[\mathrm{M}+\mathrm{H}]^{+}: 466.1829$ found 466.1819.

\section{3-3. Synthesis of $N$-tosylhydrazone 6}

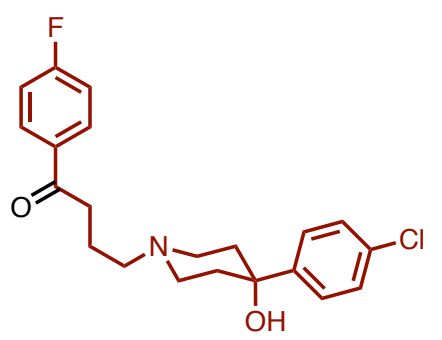

$3.0 \mathrm{mmol}$

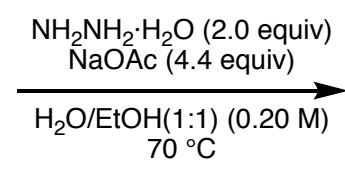

$70^{\circ} \mathrm{C}$

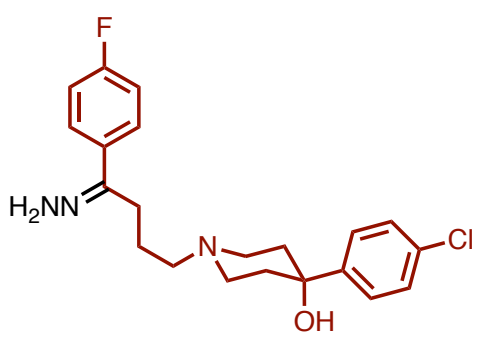

S1

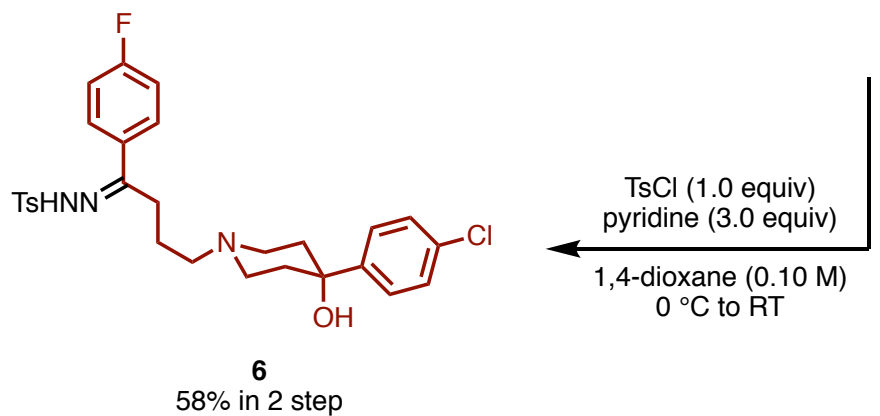

To a solution of haloperidol $\left(1.13 \mathrm{~g}, 3.0 \mathrm{mmol}, 1.0\right.$ equiv) in $\mathrm{H}_{2} \mathrm{O} / \mathrm{EtOH}(1: 1,3.0 \mathrm{~mL})$ were added hydrazine monohydrate $\left(\mathrm{NH}_{2} \mathrm{NH}_{2} \cdot \mathrm{H}_{2} \mathrm{O}: 297 \mu \mathrm{L}, 6.0 \mathrm{mmol}, 2.0\right.$ equiv) and sodium acetate (1.08 g, $13.2 \mathrm{mmol}, 4.4$ equiv). After stirring the mixture for several hours with monitoring the reaction progress by TLC, EtOAc was added to the resulting mixture to quench the reaction. The mixture was extracted three times with EtOAc. The combined organic layer was dried over $\mathrm{Na}_{2} \mathrm{SO}_{4}$, filtrated, and concentrated in vacuo to afford S1 (1.19 g, quant) as a white solid. The precipitate S1 was used without further purification. 
A solution of $\mathbf{S 1}(1.19 \mathrm{~g}, 3.05 \mathrm{mmol}, 1.0$ equiv) in 1,4-dioxane (30 mL) were added p-toluenesulfonyl chloride (TsCl: $571 \mathrm{mg}, 3.00 \mathrm{mmol}, 1.0$ equiv) and pyridine $(0.725 \mathrm{~mL}, 9.00 \mathrm{mmol}$, 3.0 equiv) at $0{ }^{\circ} \mathrm{C}$. After stirring the mixture for several hours with monitoring the reaction progress by TLC, $\mathrm{NaHCO}_{3}$ aq. was added to the resulting mixture to quench the reaction. The mixture was extracted three times with $\mathrm{CH}_{2} \mathrm{Cl}_{2}$. The combined organic layer was dried over $\mathrm{MgSO}_{4}$, filtrated, and concentrated in vacuo. Purification by Isolera ${ }^{\circledR}$ (hexane/EtOAc $=9: 1$ to 1:1) afforded 6 as a white solid (943 mg, $3.00 \mathrm{mmol} \mathrm{scale,} \mathrm{58 \%} \mathrm{yield} \mathrm{over} 2$ steps).

6: ${ }^{1} \mathrm{H}$ NMR (400 MHz, $\left.\mathrm{CDCl}_{3}\right) \delta 7.92(\mathrm{~d}, J=8.0 \mathrm{~Hz}, 2 \mathrm{H}), 7.70(\mathrm{dd}, J=8.8,5.2 \mathrm{~Hz}, 2 \mathrm{H}), 7.63(\mathrm{~d}, J=$ $8.8 \mathrm{~Hz}, 2 \mathrm{H}), 7.38$ (d, $J=8.0 \mathrm{~Hz}, 2 \mathrm{H}), 7.30$ (d, $J=8.0 \mathrm{~Hz}, 2 \mathrm{H}), 7.04$ (dd, $J=8.8,8.4 \mathrm{~Hz}, 2 \mathrm{H}), 2.86-$ $2.81(\mathrm{~m}, 2 \mathrm{H}), 2.75(\mathrm{~d}, J=13.2 \mathrm{~Hz}, 2 \mathrm{H}), 2.63(\mathrm{ddd}, J=13.2,12.0,4.0 \mathrm{~Hz}, 2 \mathrm{H}), 2.48-2.40(\mathrm{~m}, 5 \mathrm{H})$, $2.16(\mathrm{t}, J=6.0 \mathrm{~Hz}, 2 \mathrm{H}), 1.93-1.86(\mathrm{~m}, 2 \mathrm{H}), 1.73(\mathrm{dd}, J=12.8,4.0 \mathrm{~Hz}, 2 \mathrm{H}), 1.47(\mathrm{~s}, 1 \mathrm{H}) ;{ }^{13} \mathrm{C}$ NMR $\left(101 \mathrm{MHz}, \mathrm{CDCl}_{3}\right) \delta 163.5\left(\mathrm{~d}, J_{\mathrm{C}-\mathrm{F}}=248.9 \mathrm{~Hz}\right), 153.5,146.5,143.3,136.6,132.8,132.2\left(\mathrm{~d}, J_{\mathrm{C}-\mathrm{F}}=2.9\right.$ $\mathrm{Hz}), 129.2,128.5,128.0\left(\mathrm{~d}, J_{\mathrm{C}-\mathrm{F}}=7.6 \mathrm{~Hz}\right), 127.8,126.2,115.4\left(\mathrm{~d}, J_{\mathrm{C}-\mathrm{F}}=21.9 \mathrm{~Hz}\right) 71.2,54.1,48.4$, 37.3, 23.2, 22.7, 21.5; HRMS (ESI) $m / z$ calcd for $\mathrm{C}_{28} \mathrm{H}_{32} \mathrm{ClFN}_{3} \mathrm{O}_{3} \mathrm{~S}[\mathrm{M}+\mathrm{H}]^{+}: 544.1831$ found 544.1824 . 


\section{Pd-Catalyzed Alkenyl Sulfide Synthesis}<smiles>O=C(Sc1ccccc1)c1ccccc1</smiles>

$10.40 \mathrm{mmol}$

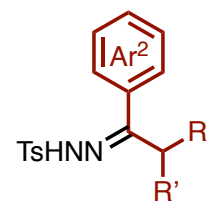

22.0 equiv

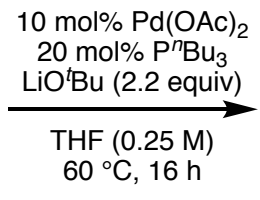

$60^{\circ} \mathrm{C}, 16 \mathrm{~h}$

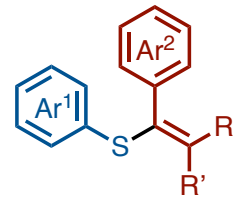

3

\section{General Procedure}

A 20-mL glass vessel equipped with J. Young ${ }^{\circledR}$ O-ring tap containing a magnetic stirring bar was dried with a heat-gun in vacuo and filled with $\mathrm{N}_{2}$ after cooling to room temperature. To this vessel were added $\mathrm{Pd}(\mathrm{OAc})_{2}(9.0 \mathrm{mg}, 0.040 \mathrm{mmol}, 10 \mathrm{~mol} \%)$, LiO ${ }^{t} \mathrm{Bu}(70.5 \mathrm{mg}, 0.88 \mathrm{mmol}, 2.2$ equiv), thioester 1 ( $0.40 \mathrm{mmol}, 1.0$ equiv) and $N$-tosylhydrazone 2 ( $0.80 \mathrm{mmol}, 2.0$ equiv). The vessel was vacuumed and refilled $\mathrm{N}_{2}$ gas three times, and then added $\mathrm{P}^{n} \mathrm{Bu}_{3}(19.0 \mu \mathrm{L}, 0.040 \mathrm{mmol}, 20 \mathrm{~mol} \%)$ and THF $(1.6 \mathrm{~mL})$. The vessel was sealed with O-ring tap and then heated at $60{ }^{\circ} \mathrm{C}$ for $16 \mathrm{~h}$ with stirring. After cooling the reaction mixture to room temperature, the mixture was passed through a short silica-gel pad with EtOAc as an eluent, and then concentrated in vacuo. The residue was purified by PTLC or Isolera ${ }^{\circledR}$ to afford the corresponding alkenyl sulfide 3.

\section{Photographic Guideline}

1. A glass vessel and a magnetic stirring bar (Photo A).

2. A vessel containing thioester, $N$-tosylhydrazone, $\mathrm{LiO}^{t} \mathrm{Bu}$ and $\mathrm{Pd}(\mathrm{OAc})_{2}$ (Photo B).

3. All materials $\left(\mathrm{P}^{n} \mathrm{Bu}_{3}\right.$ and THF) were added (Photo $\mathrm{C}$ ).

4. The vessel was heated with stirring (heated using IKA RCT-Digital and an IKA 9-well aluminum reaction block: Photo D). Color changing 2 hours later, 3.5 hour later, 6.5 hours later (Photo E, F, G).

5. The reaction finished after 16 hours (Photo $\mathrm{H})$. 

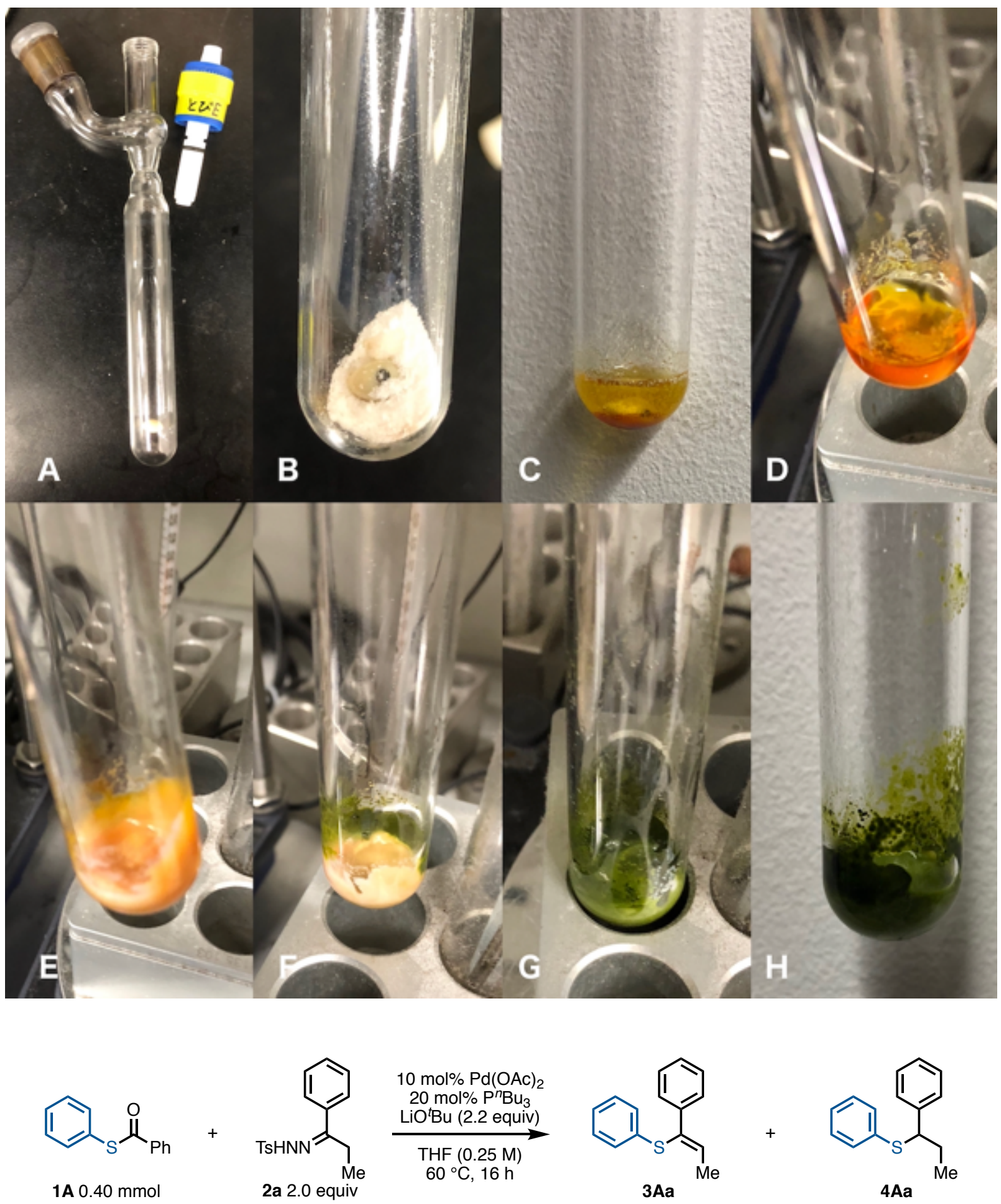

(Z)-Phenyl(1-phenylprop-1-en-1-yl)sulfane (3Aa) ${ }^{[11]}$

Crude ${ }^{1} \mathrm{H}$ NMR analysis showed 3Aa:4Aa $=90: 10, Z: E=95: 5 ;{ }^{1} \mathrm{H}$ NMR peaks at $6.52 \mathrm{ppm}(\mathrm{q}, 0.92 \mathrm{H})$ and $6.23 \mathrm{ppm}(\mathrm{q}, 0.08 \mathrm{H})$ were used. Purification by Isolera ${ }^{\circledR}$ (hexane) afforded 3Aa as a colorless oil (79.0 mg, 87\% yield, as a mixture of diasteroisomers; $Z: E=90: 10$ ).

For $Z$ isomer: ${ }^{1} \mathrm{H}$ NMR $\left(400 \mathrm{MHz}, \mathrm{CDCl}_{3}\right) \delta 7.55(\mathrm{~d}, J=6.8 \mathrm{~Hz}, 2 \mathrm{H}), 7.26-7.09(\mathrm{~m}, 7 \mathrm{H}), 7.06-7.00$ $(\mathrm{m}, 1 \mathrm{H}), 6.52(\mathrm{q}, J=6.8 \mathrm{~Hz}, 1 \mathrm{H}), 2.09(\mathrm{~d}, J=6.8 \mathrm{~Hz}, 3 \mathrm{H}) ;{ }^{13} \mathrm{C} \mathrm{NMR}\left(101 \mathrm{MHz}, \mathrm{CDCl}_{3}\right) \delta 140.5$, 
136.0, 134.6, 134.1, 128.6, 128.1, 127.8, 127.4, 127.3, 125.2, 16.9; HRMS (DART) $\mathrm{m} / \mathrm{z}$ calcd for $\mathrm{C}_{15} \mathrm{H}_{15} \mathrm{~S}[\mathrm{M}+\mathrm{H}]^{+}: 227.0889$ found 227.0888 .

For $E$ isomer: ${ }^{1} \mathrm{H}$ NMR (400 MHz, $\left.\mathrm{CDCl}_{3}\right) \delta 7.38-7.33(\mathrm{~m}, 4 \mathrm{H}), 7.26-7.09(\mathrm{~m}, 6 \mathrm{H}), 6.23(\mathrm{q}, J=6.8$ $\mathrm{Hz}, 1 \mathrm{H}), 1.78(\mathrm{~d}, J=6.8 \mathrm{~Hz}, 3 \mathrm{H})$.

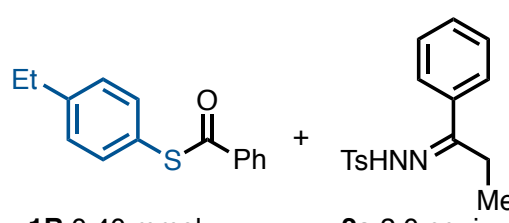

1B $0.40 \mathrm{mmol}$ 2a 2.0 equiv

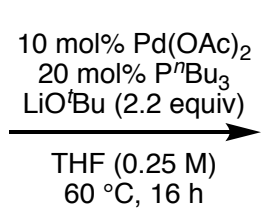

$60^{\circ} \mathrm{C}, 16 \mathrm{~h}$

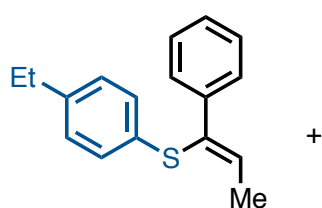

3Ba<smiles>CCc1ccc(SC(C[W])c2ccccc2)cc1</smiles>

4Ba

\section{(Z)-(4-Ethylphenyl)(1-phenylprop-1-en-1-yl)sulfane (3Ba)}

Crude ${ }^{1} \mathrm{H}$ NMR analysis showed 3Ba:4Ba $=93: 7, Z: E=93: 7 ;{ }^{1} \mathrm{H}$ NMR peaks at $6.46 \mathrm{ppm}(\mathrm{q}, 0.93 \mathrm{H})$ and $6.09 \mathrm{ppm}(\mathrm{q}, 0.07 \mathrm{H})$ were used. Purification by Isolera ${ }^{\circledR}$ (hexane) afforded 3Ba as a colorless oil (81.4 mg, 80\% yield, as a mixture of diasteroisomers; $Z: E=93: 7)$.

For $Z$ isomer: ${ }^{1} \mathrm{H}$ NMR $\left(400 \mathrm{MHz}, \mathrm{CDCl}_{3}\right) \delta 7.54(\mathrm{~d}, J=6.8 \mathrm{~Hz}, 2 \mathrm{H}), 7.27-7.12(\mathrm{~m}, 3 \mathrm{H}), 7.07(\mathrm{~d}, J=$ $8.0 \mathrm{~Hz}, 2 \mathrm{H}), 6.95(\mathrm{~d}, J=8.0 \mathrm{~Hz}, 2 \mathrm{H}), 6.46$ (q, $J=6.8 \mathrm{~Hz}, 1 \mathrm{H}), 2.54-2.46(\mathrm{~m}, 2 \mathrm{H}), 2.07$ (d, $J=6.8 \mathrm{~Hz}$, $3 \mathrm{H}), 1.17-1.10(\mathrm{~m}, 3 \mathrm{H}) ;{ }^{13} \mathrm{C}$ NMR $\left(101 \mathrm{MHz}, \mathrm{CDCl}_{3}\right) \delta 141.4,140.7,134.5,134.2,132.5,128.2$, 128.1, 128.0, 127.32, 127.28, 28.2, 16.9, 15.4; HRMS (DART) $\mathrm{m} / z$ calcd for $\mathrm{C}_{17} \mathrm{H}_{19} \mathrm{~S}[\mathrm{M}+\mathrm{H}]^{+}$: 255.1202 found 255.1202 .

For $E$ isomer: ${ }^{1} \mathrm{H}$ NMR $\left(400 \mathrm{MHz}, \mathrm{CDCl}_{3}\right) \delta 7.35(\mathrm{~d}, J=6.8 \mathrm{~Hz}, 2 \mathrm{H}), 7.25-7.12(\mathrm{~m}, 1 \mathrm{H}), 7.01(\mathrm{~d}, J=$ $8.0 \mathrm{~Hz}, 2 \mathrm{H}), 6.09$ (q, $J=6.8 \mathrm{~Hz}, 1 \mathrm{H}), 2.54-2.46(\mathrm{~m}, 2 \mathrm{H}), 1.72$ (d, $J=6.8 \mathrm{~Hz}, 3 \mathrm{H}), 1.17-1.10$ (m, 3H).

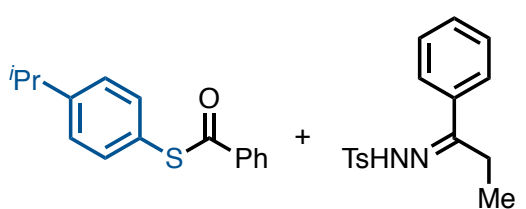

$1 \mathrm{C} 0.40 \mathrm{mmol}$

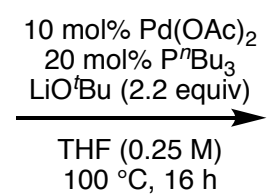

$100{ }^{\circ} \mathrm{C}, 16 \mathrm{~h}$

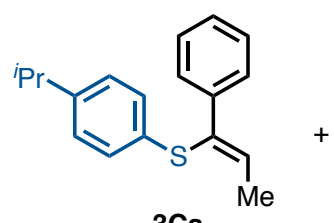

$3 \mathrm{Ca}$<smiles>CCCc1ccccc1SC(CC)c1ccc(Br)cc1</smiles>

$4 \mathrm{Ca}$

\section{(Z)-(4-Isopropylphenyl)(1-phenylprop-1-en-1-yl)sulfane (3Ca)}

The catalytic reaction was conducted at $100{ }^{\circ} \mathrm{C}$. Crude ${ }^{1} \mathrm{H}$ NMR analysis showed 3Ca:4Ca $=92: 8, Z: E$ $=92: 8 ;{ }^{1} \mathrm{H}$ NMR peaks at $6.48 \mathrm{ppm}(\mathrm{q}, 0.92 \mathrm{H})$ and $6.09 \mathrm{ppm}(\mathrm{q}, 0.08 \mathrm{H})$ were used. Purification by PTLC (hexane) afforded 3Ca as a colorless oil ( $91.4 \mathrm{mg}, 85 \%$ yield, as a mixture of diasteroisomers; $Z: E=94: 6)$.

For $Z$ isomer: ${ }^{1} \mathrm{H}$ NMR $\left(400 \mathrm{MHz}, \mathrm{CDCl}_{3}\right) \delta 7.55(\mathrm{~d}, J=7.6 \mathrm{~Hz}, 2 \mathrm{H}), 7.27-7.12(\mathrm{~m}, 3 \mathrm{H}), 7.07(\mathrm{~d}, J=$ $8.0 \mathrm{~Hz}, 2 \mathrm{H}), 6.98(\mathrm{~d}, J=8.0 \mathrm{~Hz}, 2 \mathrm{H}), 6.48(\mathrm{q}, J=6.8 \mathrm{~Hz}, 1 \mathrm{H}), 2.81-2.70(\mathrm{~m}, 1 \mathrm{H}), 2.06(\mathrm{~d}, J=6.8 \mathrm{~Hz}$, $3 \mathrm{H}), 1.17-1.12(\mathrm{~m}, 6 \mathrm{H}) ;{ }^{13} \mathrm{C} \mathrm{NMR}\left(101 \mathrm{MHz}, \mathrm{CDCl}_{3}\right) \delta 146.0,140.8,134.4,134.3,132.7,128.1$, $127.8,127.3,126.8,33.5,23.8,16.9$ (one peak is missing due to overlapping.); HRMS (ESI) $\mathrm{m} / \mathrm{z}$ calcd for $\mathrm{C}_{18} \mathrm{H}_{21} \mathrm{~S}[\mathrm{M}+\mathrm{H}]^{+}: 269.1358$ found 269.1358 . 
For $E$ isomer: ${ }^{1} \mathrm{H}$ NMR (400 MHz, $\left.\mathrm{CDCl}_{3}\right) \delta 7.35(\mathrm{~m}, 2 \mathrm{H}), 7.27-7.12(\mathrm{~m}, 7 \mathrm{H}), 6.09(\mathrm{q}, J=7.2 \mathrm{~Hz}$, $1 \mathrm{H}), 2.81-2.70(\mathrm{~m}, 1 \mathrm{H}), 1.72(\mathrm{~d}, J=7.2 \mathrm{~Hz}, 3 \mathrm{H}), 1.17-1.12(\mathrm{~m}, 6 \mathrm{H})$.

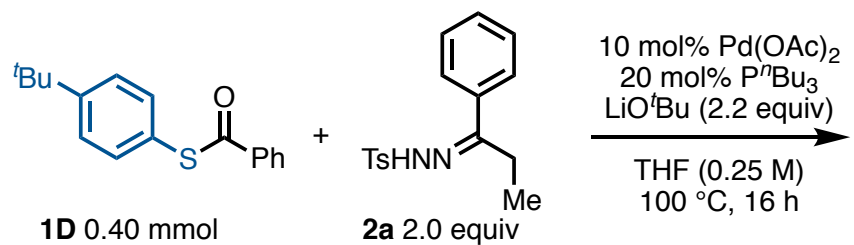

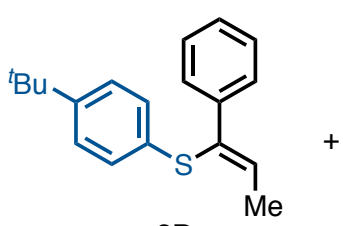

3Da<smiles>CCC(Sc1ccc(Br)cc1)c1ccccc1</smiles>

4Da

\section{(Z)-(4-(tert-Butyl)phenyl)(1-phenylprop-1-en-1-yl)sulfane (3Da)}

The catalytic reaction was conducted at $100{ }^{\circ} \mathrm{C}$. Crude ${ }^{1} \mathrm{H}$ NMR analysis showed 3Da:4Da $=95: 5, Z: E$ $=95: 5 ;{ }^{1} \mathrm{H}$ NMR peaks at $6.50 \mathrm{ppm}(\mathrm{q}, 0.95 \mathrm{H})$ and $6.10 \mathrm{ppm}(\mathrm{q}, 0.05 \mathrm{H})$ were used. Purification by PTLC (hexane) afforded 3Da as a white solid $(90.9 \mathrm{mg}, 80 \%$ yield, as a mixture of diasteroisomers; $Z: E=95: 5)$.

For $Z$ isomer: ${ }^{1} \mathrm{H}$ NMR (400 MHz, $\left.\mathrm{CDCl}_{3}\right) \delta 7.56(\mathrm{~d}, J=7.6 \mathrm{~Hz}, 2 \mathrm{H}), 7.32-7.18(\mathrm{~m}, 2 \mathrm{H}), 7.17-7.12$ $(\mathrm{m}, 3 \mathrm{H}), 7.07(\mathrm{~d}, J=8.4 \mathrm{~Hz}, 2 \mathrm{H}), 6.50(\mathrm{q}, J=6.8 \mathrm{~Hz}, 1 \mathrm{H}), 2.06(\mathrm{~d}, J=6.8 \mathrm{~Hz}, 3 \mathrm{H}), 1.21(\mathrm{~s}, 9 \mathrm{H}) ;{ }^{13} \mathrm{C}$ NMR $\left(101 \mathrm{MHz}, \mathrm{CDCl}_{3}\right) \delta 148.2,140.8,134.7,134.1,132.5,128.1,127.34,127.30,127.26,125.7$, 34.2, 31.2, 16.9; HRMS (DART) $m / z$ calcd for $\mathrm{C}_{19} \mathrm{H}_{23} \mathrm{~S}[\mathrm{M}+\mathrm{H}]^{+}: 283.1515$ found 283.1511.

For $E$ isomer: ${ }^{1} \mathrm{H}$ NMR $\left(400 \mathrm{MHz}, \mathrm{CDCl}_{3}\right) \delta 7.38-7.18(\mathrm{~m}, 9 \mathrm{H}), 6.10(\mathrm{q}, J=7.2 \mathrm{~Hz}, 1 \mathrm{H}), 1.72(\mathrm{~d}, J=$ $7.2 \mathrm{~Hz}, 3 \mathrm{H}), 1.24(\mathrm{~s}, 9 \mathrm{H})$.

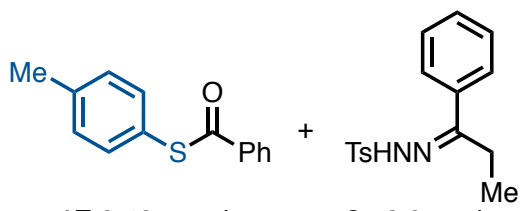

$1 \mathrm{E} 0.40 \mathrm{mmol}$

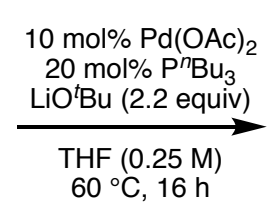

$60^{\circ} \mathrm{C}, 16 \mathrm{~h}$

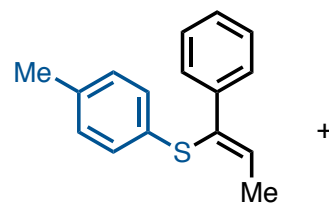

3Ea

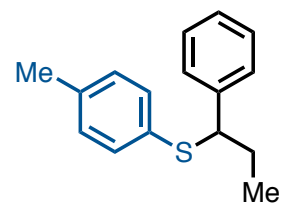

4Ea

\section{(Z)-(1-Phenylprop-1-en-1-yl)(p-tolyl)sulfane (3Ea) ${ }^{[11]}$}

Crude ${ }^{1} \mathrm{H}$ NMR analysis showed 3Ea:4Ea $=89: 11, Z: E=87: 13 ;{ }^{1} \mathrm{H}$ NMR peaks at $6.44 \mathrm{ppm}(\mathrm{q}$, $0.87 \mathrm{H}$ ) and $6.09 \mathrm{ppm}(\mathrm{q}, 0.13 \mathrm{H})$ were used. Purification by Isolera ${ }^{\circledR}$ (hexane) afforded 3Ea as a colorless oil (73.1 mg, 76\% yield, as a mixture of diasteroisomers; $Z: E=87: 13$ ).

For $Z$ isomer: ${ }^{1} \mathrm{H}$ NMR (400 MHz, $\left.\mathrm{CDCl}_{3}\right) \delta 7.53(\mathrm{~d}, J=7.2 \mathrm{~Hz}, 2 \mathrm{H}), 7.27-7.12(\mathrm{~m}, 3 \mathrm{H}), 7.04(\mathrm{~d}, J=$ $8.4 \mathrm{~Hz}, 2 \mathrm{H}), 6.92(\mathrm{~d}, J=8.4 \mathrm{~Hz}, 2 \mathrm{H}), 6.44(\mathrm{q}, J=6.8 \mathrm{~Hz}, 1 \mathrm{H}), 2.18(\mathrm{~s}, 3 \mathrm{H}), 2.07$ (d, $J=6.8 \mathrm{~Hz}, 3 \mathrm{H})$;

${ }^{13} \mathrm{C} \mathrm{NMR}\left(101 \mathrm{MHz}, \mathrm{CDCl}_{3}\right) \delta 140.6,135.1,134.6,134.0,132.2,129.4,128.2,128.0,127.34,127.27$, 20.9, 16.8; HRMS (DART) $\mathrm{m} / z$ calcd for $\mathrm{C}_{16} \mathrm{H}_{17} \mathrm{~S}[\mathrm{M}+\mathrm{H}]^{+}: 241.1045$ found 241.1045 .

For $E$ isomer: ${ }^{1} \mathrm{H}$ NMR $\left(400 \mathrm{MHz}, \mathrm{CDCl}_{3}\right) \delta 7.34(\mathrm{~d}, J=6.8 \mathrm{~Hz}, 2 \mathrm{H}), 7.27-7.12(\mathrm{~m}, 6 \mathrm{H}), 6.97(\mathrm{~d}, J=$ $8.0 \mathrm{~Hz}, 2 \mathrm{H}), 6.09$ (q, $J=7.2 \mathrm{~Hz}, 1 \mathrm{H}), 2.22(\mathrm{~s}, 3 \mathrm{H}), 1.72$ (d, $J=7.2 \mathrm{~Hz}, 3 \mathrm{H})$. 

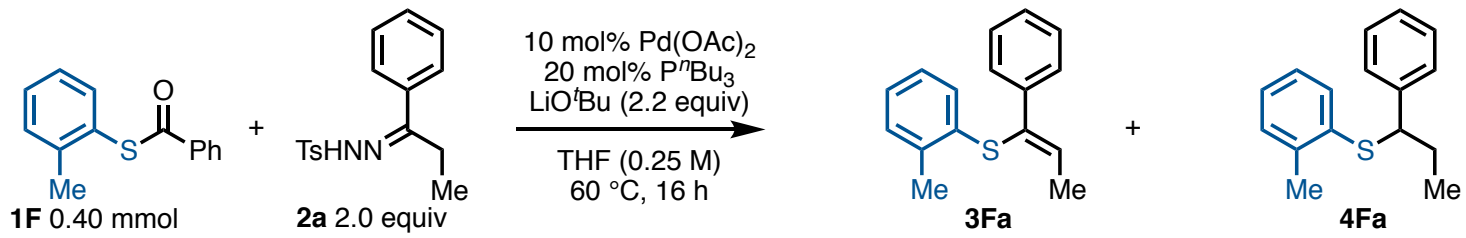

\section{(Z)-(1-Phenylprop-1-en-1-yl)(o-tolyl)sulfane (3Fa)}

Crude ${ }^{1} \mathrm{H}$ NMR analysis showed 3Fa:4Fa $=>95: 5, Z: E=97: 3 ;{ }^{1} \mathrm{H}$ NMR peaks at $6.51 \mathrm{ppm}(\mathrm{q}, 0.97 \mathrm{H})$ and $6.16 \mathrm{ppm}(\mathrm{q}, 0.03 \mathrm{H})$ were used. Purification by Isolera ${ }^{\circledR}$ (hexane) afforded $\mathbf{3 F a}$ as a colorless oil (87.2 mg, 91\% yield, as a mixture of diasteroisomers; $Z: E=97: 3$ ).

For $Z$ isomer: ${ }^{1} \mathrm{H}$ NMR (400 MHz, $\left.\mathrm{CDCl}_{3}\right) \delta 7.50(\mathrm{~d}, J=8.0 \mathrm{~Hz}, 2 \mathrm{H}), 7.38-7.11(\mathrm{~m}, 3 \mathrm{H}), 7.05(\mathrm{~d}, J=$ $6.4 \mathrm{~Hz} 1 \mathrm{H}), 7.00(\mathrm{~d}, J=6.8 \mathrm{~Hz}, 1 \mathrm{H}), 6.96-6.88(\mathrm{~m}, 2 \mathrm{H}), 6.51$ (q, $J=6.8 \mathrm{~Hz}, 1 \mathrm{H}), 2.39(\mathrm{~s}, 3 \mathrm{H}), 2.06(\mathrm{~d}$, $J=6.8 \mathrm{~Hz}, 3 \mathrm{H}) ;{ }^{13} \mathrm{C} \mathrm{NMR}\left(101 \mathrm{MHz}, \mathrm{CDCl}_{3}\right) \delta 140.6,136.4,134.8,134.5,134.0,129.9,128.1,127.8$, 127.3, 127.2, 126.1, 125.3, 20.2, 16.8; HRMS (DART) $\mathrm{m} / z$ calcd for $\mathrm{C}_{16} \mathrm{H}_{17} \mathrm{~S}[\mathrm{M}+\mathrm{H}]^{+}: 241.1045$ found 241.1045 .

For $E$ isomer: ${ }^{1} \mathrm{H}$ NMR $\left(400 \mathrm{MHz}, \mathrm{CDCl}_{3}\right) \delta 7.38-7.11(\mathrm{~m}, 10 \mathrm{H}), 6.16(\mathrm{q}, J=7.2 \mathrm{~Hz}, 1 \mathrm{H}), 2.39$ (s, $3 \mathrm{H}), 1.75(\mathrm{~d}, J=7.2 \mathrm{~Hz}, 3 \mathrm{H})$.

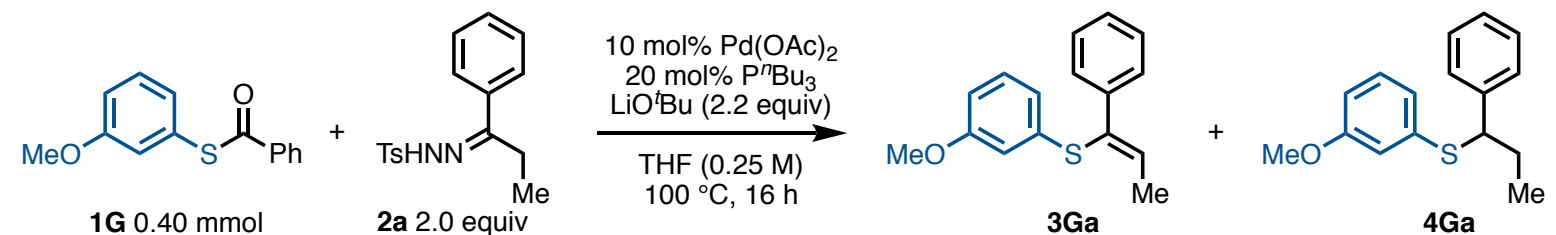

\section{(Z)-(3-Methoxyphenyl)(1-phenylprop-1-en-1-yl)sulfane (3Ga)}

The catalytic reaction was conducted at $100{ }^{\circ} \mathrm{C}$. Crude ${ }^{1} \mathrm{H}$ NMR analysis showed 3Ga:4Ga $=92: 8$, $Z: E=86: 14 ;{ }^{1} \mathrm{H}$ NMR peaks at $2.08 \mathrm{ppm}(\mathrm{q}, 2.58 \mathrm{H})$ and $1.79 \mathrm{ppm}(\mathrm{q}, 0.42 \mathrm{H})$ were used. Purification by PTLC (hexane) afforded $\mathbf{3 G a}$ as a colorless oil $(81.4 \mathrm{mg}, 79 \%$ yield, as a mixture of diasteroisomers; $Z: E=88: 12$ ).

For $Z$ isomer: ${ }^{1} \mathrm{H}$ NMR (400 MHz, $\left.\mathrm{CDCl}_{3}\right) \delta 7.55(\mathrm{~d}, J=8.4 \mathrm{~Hz}, 2 \mathrm{H}), 7.28-7.15(\mathrm{~m}, 3 \mathrm{H}), 7.04(\mathrm{t}, J=$ $8.0 \mathrm{~Hz}, 1 \mathrm{H}), 6.75$ (dd, $J=8.0,2.4 \mathrm{~Hz}, 1 \mathrm{H}), 6.68$ (d, $J=2.4 \mathrm{~Hz}, 1 \mathrm{H}), 6.57$ (dd, $J=8.0,2.4 \mathrm{~Hz}, 1 \mathrm{H})$, $6.53(\mathrm{q}, J=6.8 \mathrm{~Hz}, 1 \mathrm{H}), 3.66(\mathrm{~s}, 3 \mathrm{H}), 2.08(\mathrm{~d}, J=6.8 \mathrm{~Hz}, 3 \mathrm{H}) ;{ }^{13} \mathrm{C} \mathrm{NMR}\left(101 \mathrm{MHz}, \mathrm{CDCl}_{3}\right) \delta 159.7$, 140.7, 137.4, 135.0, 133.8, 129.4, 128.1, 127.4, 127.2, 120.0, 112.9, 111.1, 55.1, 16.9; HRMS (DART) $\mathrm{m} / z$ calcd for $\mathrm{C}_{16} \mathrm{H}_{17} \mathrm{OS}[\mathrm{M}+\mathrm{H}]^{+}: 257.0995$ found 257.0992.

For $E$ isomer: ${ }^{1} \mathrm{H}$ NMR $\left(400 \mathrm{MHz}, \mathrm{CDCl}_{3}\right) \delta 7.38(\mathrm{~d}, J=7.2 \mathrm{~Hz}, 2 \mathrm{H}), 7.28-7.15(\mathrm{~m}, 3 \mathrm{H}), 7.08-7.02$ (m, 1H), $6.84(\mathrm{ddd}, J=7.6,2.0,0.8 \mathrm{~Hz}, 1 \mathrm{H}), 6.78-6.73(\mathrm{~m}, 1 \mathrm{H}), 6.62$ (ddd, $J=8.4,2.8,0.8 \mathrm{~Hz}, 1 \mathrm{H})$, $6.29(\mathrm{q}, J=7.6 \mathrm{~Hz}, 1 \mathrm{H}), 3.68(\mathrm{~s}, 3 \mathrm{H}), 1.79(\mathrm{~d}, J=7.6 \mathrm{~Hz}, 3 \mathrm{H})$. 


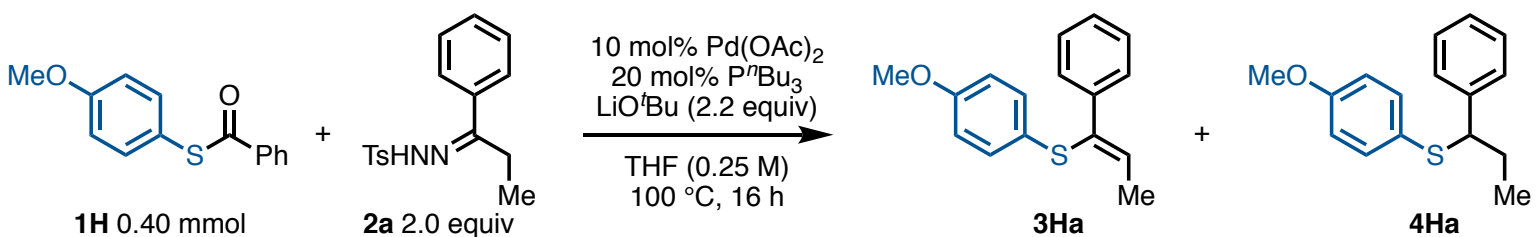

\section{(Z)-(4-Methoxyphenyl)(1-phenylprop-1-en-1-yl)sulfane (3Ha) ${ }^{[11]}$}

The catalytic reaction was conducted at $100{ }^{\circ} \mathrm{C}$. Crude ${ }^{1} \mathrm{H}$ NMR analysis showed 3Ha:4Ha $=85: 15$, $Z: E=82: 18 ;{ }^{1} \mathrm{H}$ NMR peaks at $6.33 \mathrm{ppm}(\mathrm{q}, 0.82 \mathrm{H})$ and $5.92 \mathrm{ppm}(\mathrm{q}, 0.18 \mathrm{H})$ were used. Purification by PTLC (hexane) afforded $\mathbf{3 H a}$ as a colorless solid $(64.2 \mathrm{mg}, 63 \%$ yield, as a mixture of diasteroisomers; $Z: E=83: 17)$.

For $Z$ isomer: ${ }^{1} \mathrm{H}$ NMR $\left(400 \mathrm{MHz}, \mathrm{CDCl}_{3}\right) \delta 7.48(\mathrm{~d}, J=7.2 \mathrm{~Hz}, 2 \mathrm{H}), 7.23-7.17(\mathrm{~m}, 2 \mathrm{H}), 7.17-7.13$ (m, 1H), $7.10(\mathrm{~d}, J=8.8 \mathrm{~Hz}, 2 \mathrm{H}), 6.67$ (d, $J=8.8 \mathrm{~Hz}, 2 \mathrm{H}), 6.33$ (q, $J=6.8 \mathrm{~Hz}, 1 \mathrm{H}), 3.67(\mathrm{~s}, 3 \mathrm{H}), 2.08$ $(\mathrm{d}, J=6.8 \mathrm{~Hz}, 3 \mathrm{H}) ;{ }^{13} \mathrm{C} \mathrm{NMR}\left(101 \mathrm{MHz}, \mathrm{CDCl}_{3}\right) \delta 158.0,140.6,135.8,132.5,130.9,128.0,127.5$, 127.2, 126.1, 114.3, 55.1, 16.7; HRMS (DART) $\mathrm{m} / z$ calcd for $\mathrm{C}_{16} \mathrm{H}_{17} \mathrm{OS}[\mathrm{M}+\mathrm{H}]^{+}: 257.0995$ found 257.0990 .

For $E$ isomer: ${ }^{1} \mathrm{H}$ NMR $\left(400 \mathrm{MHz}, \mathrm{CDCl}_{3}\right) \delta 7.31-7.23(\mathrm{~m}, 7 \mathrm{H}), 7.23-7.17(\mathrm{~m}, 1 \mathrm{H}), 6.74(\mathrm{~d}, J=8.8$ $\mathrm{Hz}, 2 \mathrm{H}), 5.92(\mathrm{q}, J=6.8 \mathrm{~Hz}, 1 \mathrm{H}), 3.72(\mathrm{~s}, 3 \mathrm{H}), 1.67(\mathrm{~d}, J=6.8 \mathrm{~Hz}, 3 \mathrm{H})$.

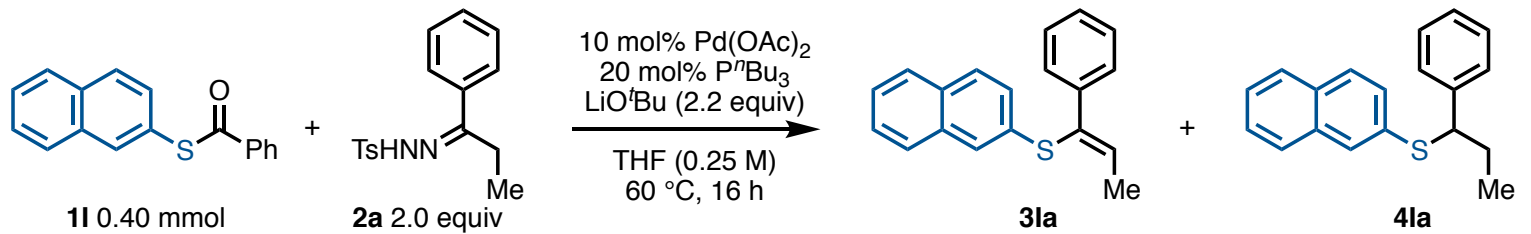

(Z)-Naphthalen-2-yl(1-phenylprop-1-en-1-yl)sulfane (3Ia)

Crude ${ }^{1} \mathrm{H}$ NMR analysis showed 3Ia:4Ia $=96: 4, Z: E=94: 6 ;{ }^{1} \mathrm{H}$ NMR peaks at $6.55 \mathrm{ppm}(\mathrm{q}, 0.94 \mathrm{H})$ and $6.26 \mathrm{ppm}(\mathrm{q}, 0.06 \mathrm{H})$ were used. Purification by Isolera ${ }^{\circledR}$ (hexane) afforded 3Ia as a white solid (97.4 $\mathrm{mg}, 88 \%$ yield, as a mixture of diasteroisomers; $Z: E=94: 6)$.

For $Z$ isomer: ${ }^{1} \mathrm{H}$ NMR (400 MHz, $\left.\mathrm{CDCl}_{3}\right) \delta 7.64(\mathrm{~d}, J=8.0 \mathrm{~Hz}, 1 \mathrm{H}), 7.60-7.55(\mathrm{~m}, 5 \mathrm{H}), 7.37-7.27$ (m, 2H), $7.24(\mathrm{dd}, J=8.0,1.6 \mathrm{~Hz}, 1 \mathrm{H}), 7.19(\mathrm{dd}, J=8.0,7.2 \mathrm{~Hz}, 2 \mathrm{H}), 7.14-7.09$ (m, 1H), 6.55 (q, $J=$ $6.8 \mathrm{~Hz}, 1 \mathrm{H}), 2.09(\mathrm{~d}, J=6.8 \mathrm{~Hz}, 3 \mathrm{H}) ;{ }^{13} \mathrm{C} \mathrm{NMR}\left(101 \mathrm{MHz}, \mathrm{CDCl}_{3}\right) \delta 140.5,134.9,133.9,133.62$, $133.57,131.3,128.2,127.6,127.4,127.2,126.9,126.3,126.1,125.6,125.2,17.0$ (one peak is missing due to overlapping.); HRMS (ESI) $m / z$ calcd for $\mathrm{C}_{19} \mathrm{H}_{17} \mathrm{~S}[\mathrm{M}+\mathrm{H}]^{+}: 277.1045$ found 277.1041.

For $E$ isomer: ${ }^{1} \mathrm{H}$ NMR $\left(400 \mathrm{MHz}, \mathrm{CDCl}_{3}\right) \delta 7.64(\mathrm{~d}, J=8.0 \mathrm{~Hz}, 1 \mathrm{H}), 7.40(\mathrm{~d}, J=7.2 \mathrm{~Hz}, 2 \mathrm{H}), 7.37-$ $7.27(\mathrm{~m}, 5 \mathrm{H}), 7.24(\mathrm{dd}, J=8.0,1.6 \mathrm{~Hz}, 1 \mathrm{H}), 7.19(\mathrm{dd}, J=8.0,7.2 \mathrm{~Hz}, 2 \mathrm{H}), 7.14-7.09(\mathrm{~m}, 1 \mathrm{H}), 6.26(\mathrm{q}$, $J=6.8 \mathrm{~Hz}, 1 \mathrm{H}), 1.77(\mathrm{~d}, J=6.8 \mathrm{~Hz}, 3 \mathrm{H})$. 


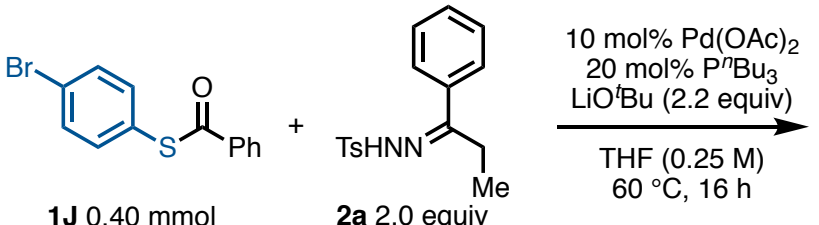<smiles>CC=C(Sc1ccc(Br)cc1)c1ccccc1</smiles><smiles>CCC(Sc1ccc(Br)cc1)c1ccccc1</smiles>

(Z)-(4-Bromophenyl)(1-phenylprop-1-en-1-yl)sulfane (3Ja)

Crude ${ }^{1} \mathrm{H}$ NMR analysis showed 3Ja:4Ja $=89: 11, Z: E=93: 7 ;{ }^{1} \mathrm{H}$ NMR peaks at $6.51 \mathrm{ppm}(\mathrm{q}, 0.93 \mathrm{H})$ and $6.28 \mathrm{ppm}(\mathrm{q}, 0.07 \mathrm{H})$ were used. Purification by PTLC (hexane) afforded 3Ja as a colorless oil (92.1 mg, 75\% yield, as a mixture of diasteroisomers; $Z: E=93: 7)$.

For $Z$ isomer: ${ }^{1} \mathrm{H}$ NMR (400 MHz, $\left.\mathrm{CDCl}_{3}\right) \delta 7.51(\mathrm{~d}, J=8.4 \mathrm{~Hz}, 2 \mathrm{H}), 7.25-7.15(\mathrm{~m}, 5 \mathrm{H}), 6.99(\mathrm{~d}, J=$ $8.8 \mathrm{~Hz}, 2 \mathrm{H}), 6.51(\mathrm{q}, J=6.8 \mathrm{~Hz}, 1 \mathrm{H}), 2.07(\mathrm{~d}, J=7.2 \mathrm{~Hz}, 3 \mathrm{H}) ;{ }^{13} \mathrm{C} \mathrm{NMR}\left(101 \mathrm{MHz}, \mathrm{CDCl}_{3}\right) \delta 140.1$, 135.3, 135.0, 133.7, 131.6, 129.4, 128.2, 127.6, 127.2, 119.0, 16.9; HRMS (ESI) $\mathrm{m} / z$ calcd for $\mathrm{C}_{15} \mathrm{H}_{14} \mathrm{BrS}[\mathrm{M}+\mathrm{H}]^{+}: 304.9994$ found 304.9992.

For $E$ isomer: ${ }^{1} \mathrm{H}$ NMR (400 MHz, $\left.\mathrm{CDCl}_{3}\right) \delta 7.34(\mathrm{~d}, J=6.8 \mathrm{~Hz}, 2 \mathrm{H}), 7.25-7.15(\mathrm{~m}, 5 \mathrm{H}), 7.08(\mathrm{~d}, J=$ $8.8 \mathrm{~Hz}, 2 \mathrm{H}), 6.28$ (q, $J=7.2 \mathrm{~Hz}, 1 \mathrm{H}), 1.78(\mathrm{~d}, J=7.2 \mathrm{~Hz}, 3 \mathrm{H})$.

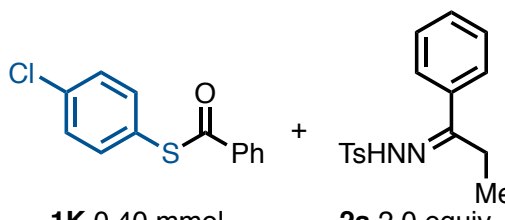

$1 \mathrm{~K} 0.40 \mathrm{mmol}$

2a 2.0 equiv

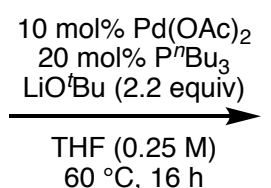

$60^{\circ} \mathrm{C}, 16 \mathrm{~h}$

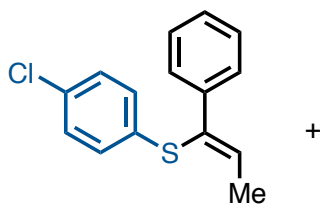

$3 \mathrm{Ka}$

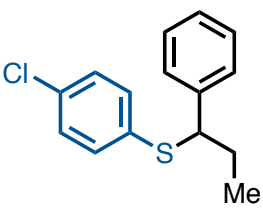

$4 \mathrm{Ka}$

(Z)-(4-Chlorophenyl)(1-phenylprop-1-en-1-yl)sulfane (3Ka) ${ }^{[11]}$

Crude ${ }^{1} \mathrm{H}$ NMR analysis showed 3Ka:4Ka $=94: 6, Z: E=94: 6 ;{ }^{1} \mathrm{H}$ NMR peaks at $6.48 \mathrm{ppm}(\mathrm{q}, 0.94 \mathrm{H})$ and $6.06 \mathrm{ppm}(\mathrm{q}, 0.06 \mathrm{H})$ were used. ${ }^{1} \mathrm{H}$ NMR yield of 8 was $48 \%\left({ }^{1} \mathrm{H}\right.$ NMR peak at $10.30 \mathrm{ppm}(\mathrm{s}, 1 \mathrm{H})$ was used). Purification by Isolera ${ }^{\circledR}$ (hexane) afforded 3Ka as a colorless oil (95.4 mg, 91\% yield, as a mixture of diasteroisomers; $Z: E=94: 6)$.

For $Z$ isomer: ${ }^{1} \mathrm{H}$ NMR (400 MHz, $\left.\mathrm{CDCl}_{3}\right) \delta 7.50(\mathrm{~d}, J=8.4 \mathrm{~Hz}, 2 \mathrm{H}), 7.24-7.13(\mathrm{~m}, 3 \mathrm{H}), 7.09-7.03$ $(\mathrm{m}, 4 \mathrm{H}), 6.48(\mathrm{q}, J=6.8 \mathrm{~Hz}, 1 \mathrm{H}), 2.06(\mathrm{~d}, J=6.8 \mathrm{~Hz}, 3 \mathrm{H}) ;{ }^{13} \mathrm{C} \mathrm{NMR}\left(101 \mathrm{MHz}, \mathrm{CDCl}_{3}\right) \delta 140.1$, 134.8, 134.5, 133.9, 131.1, 129.1, 128.7, 128.2, 127.5, 127.2, 16.9; HRMS (DART) $\mathrm{m} / z$ calcd for $\mathrm{C}_{15} \mathrm{H}_{14} \mathrm{ClS}[\mathrm{M}+\mathrm{H}]^{+}: 261.0499$ found 261.0497.

For $E$ isomer: ${ }^{1} \mathrm{H}$ NMR $\left(400 \mathrm{MHz}, \mathrm{CDCl}_{3}\right) \delta 7.36-7.33(\mathrm{~m}, 2 \mathrm{H}), 7.24-7.13(\mathrm{~m}, 7 \mathrm{H}), 6.26(\mathrm{q}, J=7.2$ $\mathrm{Hz}, 1 \mathrm{H}), 1.76(\mathrm{~d}, J=7.2 \mathrm{~Hz}, 3 \mathrm{H})$.

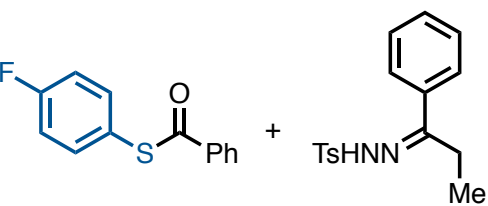

$1 \mathrm{~L} 0.40 \mathrm{mmol}$ 2a 2.0 equiv

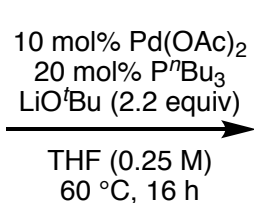

$60^{\circ} \mathrm{C}, 16 \mathrm{~h}$

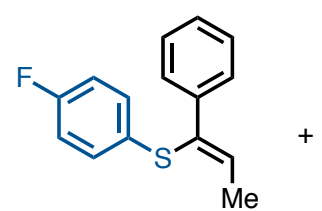

3La<smiles>O=[W]CC(Sc1ccc(F)cc1)c1ccccc1</smiles>

4La

(Z)-(4-Fluorophenyl)(1-phenylprop-1-en-1-yl)sulfane (3La) 
Crude ${ }^{1} \mathrm{H}$ NMR analysis showed 3La:4La $=91: 9, Z: E=91: 9 ;{ }^{1} \mathrm{H}$ NMR peaks at $6.41 \mathrm{ppm}(\mathrm{q}, 0.91 \mathrm{H})$ and $6.14 \mathrm{ppm}(\mathrm{q}, 0.09 \mathrm{H})$ were used. Purification by Isolera ${ }^{\circledR}$ (hexane) afforded 3La as a colorless oil (82.7 mg, $85 \%$ yield, as a mixture of diasteroisomers; $Z: E=91: 9$ ).

For $Z$ isomer: ${ }^{1} \mathrm{H}$ NMR (400 MHz, $\left.\mathrm{CDCl}_{3}\right) \delta 7.49(\mathrm{~d}, J=8.4 \mathrm{~Hz}, 2 \mathrm{H}), 7.26-7.14(\mathrm{~m}, 3 \mathrm{H}), 7.10(\mathrm{dd}, J=$ $8.8,5.6 \mathrm{~Hz}, 2 \mathrm{H}), 6.81(\mathrm{t}, J=8.8 \mathrm{~Hz}, 2 \mathrm{H}), 6.41(\mathrm{q}, J=6.8 \mathrm{~Hz}, 1 \mathrm{H}), 2.07(\mathrm{~d}, J=6.8 \mathrm{~Hz}, 3 \mathrm{H}) ;{ }^{13} \mathrm{C} \mathrm{NMR}$ $\left(101 \mathrm{MHz}, \mathrm{CDCl}_{3}\right) \delta 161.1\left(\mathrm{~d}, J_{\mathrm{C}-\mathrm{F}}=248.7 \mathrm{~Hz}\right), 140.2,134.8,133.7,130.7\left(\mathrm{~d}, J_{\mathrm{C}-\mathrm{F}}=2.9 \mathrm{~Hz}\right), 130.4(\mathrm{~d}$, $\left.J_{\mathrm{C}-\mathrm{F}}=7.7 \mathrm{~Hz}\right), 128.1,127.4,115.7\left(\mathrm{~d}, J_{\mathrm{C}-\mathrm{F}}=22.2 \mathrm{~Hz}\right.$ ), 16.8 (one peak is missing due to overlapping.); HRMS (ESI) $m / z$ calcd for $\mathrm{C}_{15} \mathrm{H}_{14} \mathrm{FS}[\mathrm{M}+\mathrm{H}]^{+}: 245.0795$ found 245.0789 .

For $E$ isomer: ${ }^{1} \mathrm{H} \mathrm{NMR}\left(400 \mathrm{MHz}, \mathrm{CDCl}_{3}\right) \delta 7.30(\mathrm{~d}, J=8.0 \mathrm{~Hz}, 2 \mathrm{H}), 7.26-7.14(\mathrm{~m}, 5 \mathrm{H}), 6.87-6.77$ (m, 2H), $6.14(\mathrm{q}, J=7.6 \mathrm{~Hz}, 1 \mathrm{H}), 1.72(\mathrm{~d}, J=7.6 \mathrm{~Hz}, 3 \mathrm{H})$.

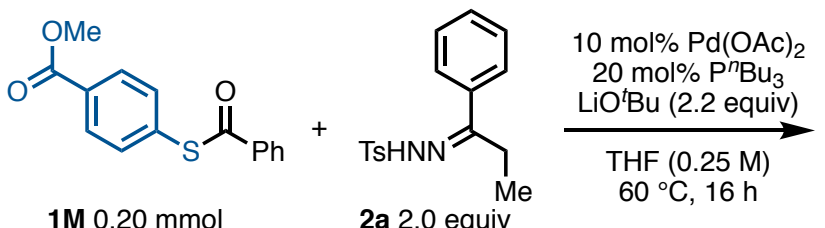

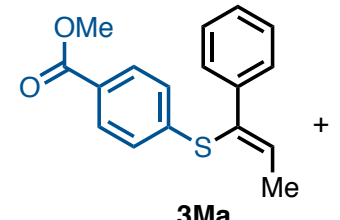

$3 \mathrm{Ma}$<smiles>COC(=O)c1ccc(SC(CO)c2ccccc2)cc1</smiles>

$4 \mathrm{Ma}$

\section{Methyl (Z)-4-((1-phenylprop-1-en-1-yl)thio)benzoate (3Ma)}

Crude ${ }^{1} \mathrm{H}$ NMR analysis showed 3Ma:4Ma $=89: 11, Z: E=93: 7 ;{ }^{1} \mathrm{H}$ NMR peaks at $6.61 \mathrm{ppm}(\mathrm{q}$, $0.93 \mathrm{H})$ and $6.46 \mathrm{ppm}(\mathrm{q}, 0.07 \mathrm{H})$ were used. Purification by PTLC (hexane/EtOAc $=19: 1)$ afforded an inseparable mixture of 3Ma and $\mathbf{4 M a}$ as a white solid $(95.7 \mathrm{mg}, Z: E=95: 5, \mathbf{3 M a}: \mathbf{4 M a}=88: 12)$. The yield of $\mathbf{3 M a}$ was determined as $74 \%$.

For $Z$ isomer: ${ }^{1} \mathrm{H}$ NMR (400 MHz, $\left.\mathrm{CDCl}_{3}\right) \delta 7.81-7.78(\mathrm{~m}, 2 \mathrm{H}), 7.54(\mathrm{~d}, J=6.8 \mathrm{~Hz}, 2 \mathrm{H}), 7.25-7.15$ $(\mathrm{m}, 5 \mathrm{H}), 6.61(\mathrm{q}, J=6.8 \mathrm{~Hz}, 1 \mathrm{H}), 3.81(\mathrm{~s}, 3 \mathrm{H}), 2.06(\mathrm{~d}, J=6.8 \mathrm{~Hz}, 3 \mathrm{H}) ;{ }^{13} \mathrm{C} \mathrm{NMR}\left(101 \mathrm{MHz}, \mathrm{CDCl}_{3}\right)$ $\delta$ 166.7, 143.0, 140.0, 136.2, 132.7, 129.8, 128.3, 127.7, 127.1, 126.6, 126.5, 51.9, 17.0; HRMS (DART) $\mathrm{m} / z$ calcd for $\mathrm{C}_{17} \mathrm{H}_{17} \mathrm{O}_{2} \mathrm{~S}[\mathrm{M}+\mathrm{H}]^{+}: 285.0944$ found 285.0940 .

For $E$ isomer: ${ }^{1} \mathrm{H}$ NMR $\left(400 \mathrm{MHz}, \mathrm{CDCl}_{3}\right) \delta$ 7.81-7.78 (m, 2H), 7.41-7.38 (m, 2H), 7.25-7.15 (m, $5 \mathrm{H}), 6.46(\mathrm{q}, J=7.2 \mathrm{~Hz}, 1 \mathrm{H}), 3.83(\mathrm{~s}, 3 \mathrm{H}), 1.85(\mathrm{~d}, J=7.2 \mathrm{~Hz}, 3 \mathrm{H})$.

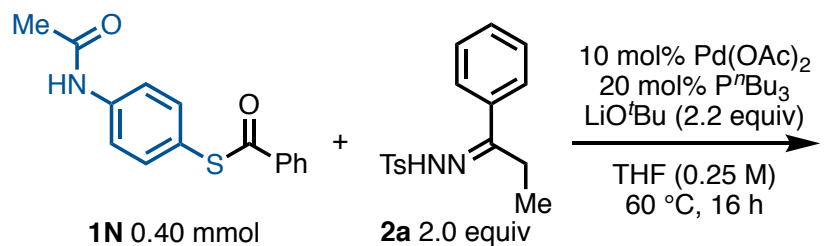

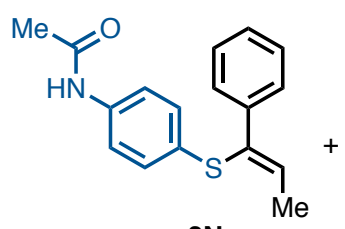

$3 \mathrm{Na}$

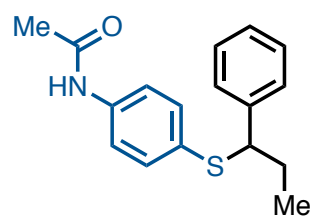

$4 \mathrm{Na}$

\section{(Z)-N-(4-((1-Phenylprop-1-en-1-yl)thio)phenyl)acetamide (3Na)}

Crude ${ }^{1} \mathrm{H}$ NMR analysis showed 3Na:4Na $=90: 10, Z: E=93: 7 ;{ }^{1} \mathrm{H}$ NMR peaks at $6.44 \mathrm{ppm}(\mathrm{q}, 0.93 \mathrm{H})$ and $6.13 \mathrm{ppm}(\mathrm{q}, 0.07 \mathrm{H})$ were used. Purification by PTLC (hexane/EtOA $=2: 1$ ) afforded a mixture of $3 \mathbf{N a}$ and $\mathbf{4 N a}$ as a white solid ( $90.0 \mathrm{mg}, \mathbf{3 N a}: \mathbf{4 N a}=93: 7)$. The yield of $\mathbf{3 N a}$ was determined as $74 \%$. 
Further purification by PTLC (hexane/EtOA $=2: 1$ ) was performed to give pure $\mathbf{3 N a}$ as a white solid for the characterization.

For $Z$ isomer: ${ }^{1} \mathrm{H}$ NMR (400 MHz, $\left.\mathrm{CDCl}_{3}\right) \delta 7.91-7.75$ (brs, $\left.1 \mathrm{H}\right), 7.51(\mathrm{~d}, J=7.2 \mathrm{~Hz}, 2 \mathrm{H}), 7.25$ (d, $J=$ $8.8 \mathrm{~Hz}, 2 \mathrm{H}), 7.24-7.14(\mathrm{~m}, 3 \mathrm{H}), 7.07$ (d, $J=8.8 \mathrm{~Hz}, 2 \mathrm{H}), 6.44$ (q, $J=6.8 \mathrm{~Hz}, 1 \mathrm{H}), 2.11(\mathrm{~s}, 3 \mathrm{H}), 2.09$ $(\mathrm{d}, J=6.8 \mathrm{~Hz}, 3 \mathrm{H}) ;{ }^{13} \mathrm{C} \mathrm{NMR}\left(101 \mathrm{MHz}, \mathrm{CDCl}_{3}\right) \delta 168.2,140.5,135.6,134.6,133.9,131.0,129.2$, $128.1,127.40,127.37,120.2,24.5,16.8$; HRMS (DART) $\mathrm{m} / z$ calcd for $\mathrm{C}_{17} \mathrm{H}_{18} \mathrm{NOS}[\mathrm{M}+\mathrm{H}]^{+}: 284.1104$ found 284.1100 .

For $E$ isomer: ${ }^{1} \mathrm{H}$ NMR (400 MHz, $\left.\mathrm{CDCl}_{3}\right) \delta 7.34-7.10(\mathrm{~m}, 10 \mathrm{H}), 6.13(\mathrm{q}, J=7.6 \mathrm{~Hz}, 1 \mathrm{H}), 2.02(\mathrm{~s}$, $3 \mathrm{H}), 1.74(\mathrm{~d}, J=7.6 \mathrm{~Hz}, 3 \mathrm{H})$.
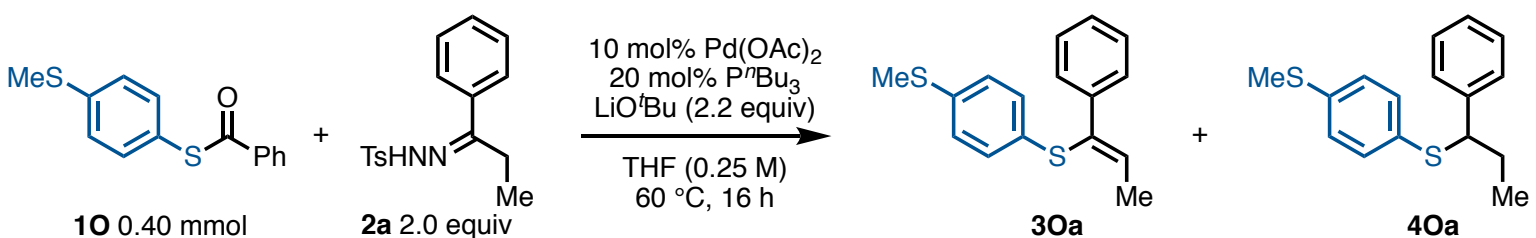

(Z)-Methyl(4-((1-phenylprop-1-en-1-yl)thio)phenyl)sulfane (3Oa)

Crude ${ }^{1} \mathrm{H}$ NMR analysis showed 3Oa:4Oa $=96: 4, Z: E=95: 5 ;{ }^{1} \mathrm{H}$ NMR peaks at $6.46 \mathrm{ppm}(\mathrm{q}, 0.95 \mathrm{H})$ and $6.15 \mathrm{ppm}(\mathrm{q}, 0.05 \mathrm{H})$ were used. Purification by Isolera ${ }^{\circledR}$ (hexane $/ \mathrm{CH}_{2} \mathrm{Cl}_{2}=99: 1$ to 9:1) afforded 30a as a colorless oil ( $94.3 \mathrm{mg}, 87 \%$ yield, as a mixture of diasteroisomers; $Z: E=95: 5$ ).

For $Z$ isomer: ${ }^{1} \mathrm{H}$ NMR (400 MHz, $\left.\mathrm{CDCl}_{3}\right) \delta 7.51(\mathrm{~d}, J=8.4 \mathrm{~Hz}, 2 \mathrm{H}), 7.24-7.18(\mathrm{~m}, 2 \mathrm{H}), 7.18-7.13$ $(\mathrm{m}, 1 \mathrm{H}), 7.06(\mathrm{~d}, J=8.4 \mathrm{~Hz}, 2 \mathrm{H}), 7.00(\mathrm{~d}, J=8.4 \mathrm{~Hz}, 2 \mathrm{H}), 6.46(\mathrm{q}, J=6.8 \mathrm{~Hz}, 1 \mathrm{H}), 2.34(\mathrm{~s}, 3 \mathrm{H}), 2.07$ $(\mathrm{d}, J=6.8 \mathrm{~Hz}, 3 \mathrm{H}) ;{ }^{13} \mathrm{C} \mathrm{NMR}\left(101 \mathrm{MHz}, \mathrm{CDCl}_{3}\right) \delta 140.3,135.3,134.3,134.2,132.5,128.6,128.1$, 127.4, 127.3, 127.0, 16.8, 15.9; HRMS (DART) $\mathrm{m} / z$ calcd for $\mathrm{C}_{16} \mathrm{H}_{17} \mathrm{~S}_{2}[\mathrm{M}+\mathrm{H}]^{+}: 273.0766$ found 273.0761 .

For $E$ isomer: ${ }^{1} \mathrm{H}$ NMR $\left(400 \mathrm{MHz}, \mathrm{CDCl}_{3}\right) \delta 7.33(\mathrm{~d}, J=6.8 \mathrm{~Hz}, 2 \mathrm{H}), 7.24-7.13(\mathrm{~m}, 7 \mathrm{H}), 6.15(\mathrm{q}, J=$ $7.2 \mathrm{~Hz}, 1 \mathrm{H}), 2.36(\mathrm{~s}, 3 \mathrm{H}), 1.74(\mathrm{~d}, J=7.2 \mathrm{~Hz}, 3 \mathrm{H})$.
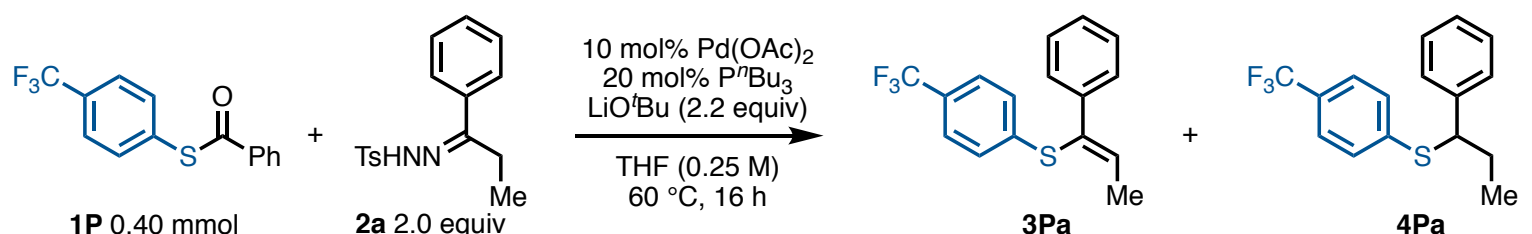

(Z)-(1-Phenylprop-1-en-1-yl)(4-(trifluoromethyl)phenyl)sulfane (3Pa)

Crude ${ }^{1} \mathrm{H}$ NMR analysis showed 3Pa:4Pa $=88: 12, Z: E=95: 5 ;{ }^{1} \mathrm{H}$ NMR peaks at $6.63 \mathrm{ppm}(\mathrm{q}, 0.95 \mathrm{H})$ and $6.45 \mathrm{ppm}(\mathrm{q}, 0.05 \mathrm{H})$ were used. Purification by Isolera ${ }^{\circledR}$ (hexane) afforded 3Pa as a colorless oil $(100.9 \mathrm{mg}, 86 \%$ yield, as a mixture of diasteroisomers; $Z: E=95: 5)$.

For $Z$ isomer: ${ }^{1} \mathrm{H}$ NMR (400 MHz, $\left.\mathrm{CDCl}_{3}\right) \delta 7.55(\mathrm{~d}, J=8.4 \mathrm{~Hz}, 2 \mathrm{H}), 7.36(\mathrm{~d}, J=8.8 \mathrm{~Hz}, 2 \mathrm{H}), 7.28-$ $7.17(\mathrm{~m}, 5 \mathrm{H}), 6.63(\mathrm{q}, J=6.8 \mathrm{~Hz}, 1 \mathrm{H}), 2.07(\mathrm{~d}, J=6.8 \mathrm{~Hz}, 3 \mathrm{H}) ;{ }^{13} \mathrm{C} \mathrm{NMR}\left(101 \mathrm{MHz}, \mathrm{CDCl}_{3}\right) \delta 141.5$, 
$140.0,136.5,132.6,128.4,128.2\left(\mathrm{q}, J_{\mathrm{C}-\mathrm{F}}=33.8 \mathrm{~Hz}\right), 127.8,127.1,126.9,125.5\left(\mathrm{q}, J_{\mathrm{C}-\mathrm{F}}=2.9 \mathrm{~Hz}\right)$, $124.1\left(\mathrm{q}, J_{\mathrm{C}-\mathrm{F}}=275.8 \mathrm{~Hz}\right.$ ), 17.0; HRMS (DART) $\mathrm{m} / z$ calcd for $\mathrm{C}_{16} \mathrm{H}_{14} \mathrm{~F}_{3} \mathrm{~S}[\mathrm{M}+\mathrm{H}]^{+}: 294.0685$ found 294.0679 .

For $E$ isomer: ${ }^{1} \mathrm{H}$ NMR (400 MHz, $\left.\mathrm{CDCl}_{3}\right) \delta 7.38-7.33(\mathrm{~d}, 2 \mathrm{H}), 7.28-7.17(\mathrm{~m}, 7 \mathrm{H}), 6.45(\mathrm{q}, J=7.2$ $\mathrm{Hz}, 1 \mathrm{H}), 1.84(\mathrm{~d}, J=7.2 \mathrm{~Hz}, 3 \mathrm{H})$.
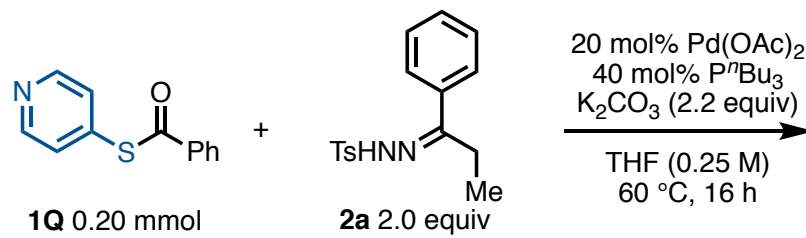

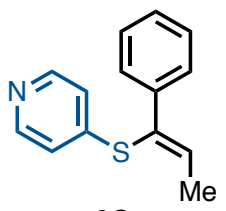

3Qa

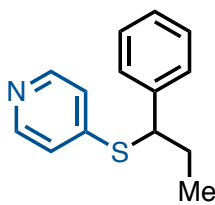

4Qa

\section{(Z)-4-((1-Phenylprop-1-en-1-yl)thio)pyridine (3Qa)}

The catalytic reaction $\left(0.20 \mathrm{mmol}\right.$ scale) was conducted by using 3Qa (1.5 equiv), $\mathrm{Pd}(\mathrm{OAc})_{2}(20$ mol\%), $\mathrm{P}^{n} \mathrm{Bu}_{3}$ (40 mol\%, and $\mathrm{K}_{2} \mathrm{CO}_{3}$ (2.2 equiv). Crude ${ }^{1} \mathrm{H}$ NMR analysis showed 3Qa:4Qa $=90: 10$, $Z: E=94: 6 ;{ }^{1} \mathrm{H}$ NMR peaks at $6.73 \mathrm{ppm}(\mathrm{q}, 0.94 \mathrm{H})$ and $6.56 \mathrm{ppm}(\mathrm{q}, 0.06 \mathrm{H})$ were used. Purification by PTLC (hexane/EtOAc $=4: 1)$ afforded 3Qa as a white solid $(39.8 \mathrm{mg}, 88 \%$ yield, as a mixture of diasteroisomers; $Z: E=94: 6)$.

For $Z$ isomer: ${ }^{1} \mathrm{H}$ NMR $\left(400 \mathrm{MHz}, \mathrm{CDCl}_{3}\right) \delta 8.28(\mathrm{~d}, J=6.4 \mathrm{~Hz}, 2 \mathrm{H}), 7.56(\mathrm{~d}, J=8.0 \mathrm{~Hz}, 2 \mathrm{H}), 7.32$ $7.21(\mathrm{~m}, 3 \mathrm{H}), 7.02(\mathrm{~d}, J=6.4 \mathrm{~Hz}, 2 \mathrm{H}), 6.73(\mathrm{q}, J=6.8 \mathrm{~Hz}, 1 \mathrm{H}), 2.07(\mathrm{~d}, J=6.8 \mathrm{~Hz}, 3 \mathrm{H}) ;{ }^{13} \mathrm{C} \mathrm{NMR}$ $\left(101 \mathrm{MHz}, \mathrm{CDCl}_{3}\right) \delta 149.2,148.1,139.8,137.6,131.1,128.5,127.9,126.9,121.0,17.0$; HRMS (DART) $\mathrm{m} / z$ calcd for $\mathrm{C}_{14} \mathrm{H}_{14} \mathrm{NS}[\mathrm{M}+\mathrm{H}]^{+}: 228.0841$ found 228.0837.

For $E$ isomer: ${ }^{1} \mathrm{H}$ NMR (400 MHz, $\left.\mathrm{CDCl}_{3}\right) \delta 8.32(\mathrm{~d}, J=6.4 \mathrm{~Hz}, 2 \mathrm{H}), 7.44(\mathrm{~d}, J=8.4 \mathrm{~Hz}, 2 \mathrm{H}), 7.32-$ $7.21(\mathrm{~m}, 3 \mathrm{H}), 6.91(\mathrm{~d}, J=6.4 \mathrm{~Hz}, 2 \mathrm{H}), 6.56(\mathrm{q}, J=7.2 \mathrm{~Hz}, 1 \mathrm{H}), 1.92(\mathrm{~d}, J=7.2 \mathrm{~Hz}, 3 \mathrm{H})$. 


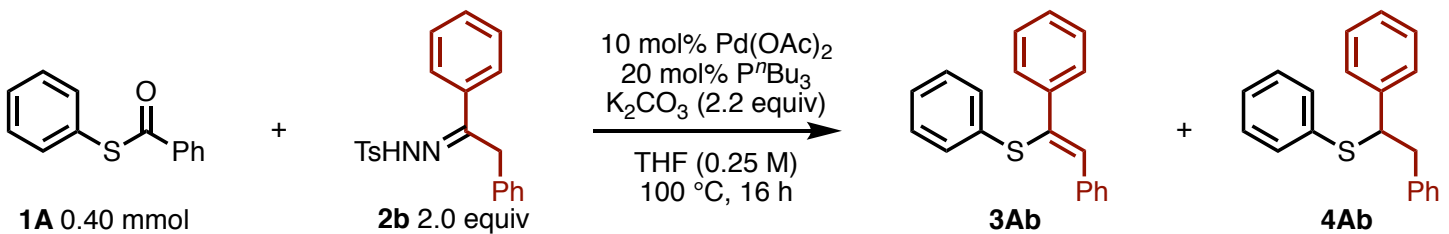

\section{(Z)-(1,2-Diphenylvinyl)(phenyl)sulfane (3Ab) ${ }^{[12]}$}

The catalytic reaction was conducted by using $\mathrm{K}_{2} \mathrm{CO}_{3}$ instead of $\mathrm{LiO}^{t} \mathrm{Bu}$ at $100{ }^{\circ} \mathrm{C}$. Crude ${ }^{1} \mathrm{H}$ NMR analysis showed 3Ab:4Ab $=78: 22, Z: E=88: 12 ;{ }^{1} \mathrm{H}$ NMR peaks at $7.72 \mathrm{ppm}(\mathrm{d}, 1.76 \mathrm{H})$ and 6.79 ppm (s, 0.12H) were used. Purification by PTLC (hexane) afforded 3Ab as a white solid (74.9 mg, $65 \%$ yield, as a mixture of diasteroisomers; $Z: E=88: 12$ ).

For $Z$ isomer: ${ }^{1} \mathrm{H}$ NMR $\left(400 \mathrm{MHz}, \mathrm{CDCl}_{3}\right) \delta 7.72(\mathrm{~d}, J=7.2 \mathrm{~Hz}, 2 \mathrm{H}), 7.63(\mathrm{~d}, J=7.6 \mathrm{~Hz}, 2 \mathrm{H}), 7.35$ $(\mathrm{dd}, J=7.6,7.2 \mathrm{~Hz}, 2 \mathrm{H}), 7.28-7.15(\mathrm{~m}, 7 \mathrm{H}), 7.08(\mathrm{t}, J=7.6 \mathrm{~Hz}, 2 \mathrm{H}), 7.00(\mathrm{t}, J=7.2 \mathrm{~Hz}, 1 \mathrm{H}) ;{ }^{13} \mathrm{C}$ NMR $\left(101 \mathrm{MHz}, \mathrm{CDCl}_{3}\right) \delta 140.9,136.7,135.6,135.2,134.6,129.5,129.1,128.6,128.14,128.12$, 128.0, 127.9, 127.8, 125.8; HRMS (ESI) $m / z$ calcd for $\mathrm{C}_{20} \mathrm{H}_{17} \mathrm{~S}[\mathrm{M}+\mathrm{H}]^{+}: 289.1045$ found 289.1048 .

For $E$ isomer: ${ }^{1} \mathrm{H}$ NMR $\left(400 \mathrm{MHz}, \mathrm{CDCl}_{3}\right) \delta$ 7.41-7.32 (m, 4H), 7.28-7.15 (m, 6H), 7.12-7.06 (m, $3 \mathrm{H}), 6.96-6.92(\mathrm{~m}, 2 \mathrm{H}), 6.79(\mathrm{~s}, 1 \mathrm{H})$.

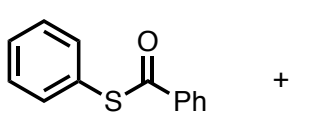

$1 \mathrm{~A} 0.40 \mathrm{mmol}$

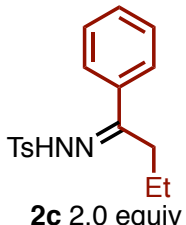

2c 2.0 equiv

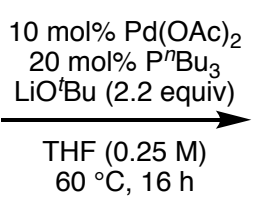

$60^{\circ} \mathrm{C}, 16 \mathrm{~h}$<smiles>CC=C(Sc1ccccc1)c1ccccc1</smiles>

3Ac

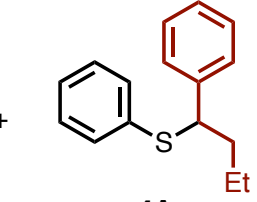

4AC

\section{(Z)-Phenyl(1-phenylbut-1-en-1-yl)sulfane) (3Ac) ${ }^{[11]}$}

Crude ${ }^{1} \mathrm{H}$ NMR analysis showed 3Ac:4Ac $=93: 7, Z: E=93: 7 ;{ }^{1} \mathrm{H}$ NMR peaks at $6.41 \mathrm{ppm}(\mathrm{q}, 0.93 \mathrm{H})$ and $6.13 \mathrm{ppm}(\mathrm{q}, 0.07 \mathrm{H})$ were used. Purification by PTLC (hexane) afforded 3Ac as a colorless oil (82.3 $\mathrm{mg}, 86 \%$ yield, as a mixture of diasteroisomers; $Z: E=93: 7)$.

For $Z$ isomer: ${ }^{1} \mathrm{H}$ NMR (400 MHz, $\left.\mathrm{CDCl}_{3}\right) \delta 7.55(\mathrm{~d}, J=7.2 \mathrm{~Hz}, 2 \mathrm{H}), 7.20(\mathrm{dd}, J=7.6,7.2 \mathrm{~Hz}, 2 \mathrm{H})$, 7.16-7.06 (m, 5H), 6.98 (t, $J=6.8 \mathrm{~Hz}, 1 \mathrm{H}), 6.41(\mathrm{t}, J=7.2 \mathrm{~Hz}, 1 \mathrm{H}), 2.57-2.48(\mathrm{~m}, 2 \mathrm{H}), 1.08$ (t, $J=$ $8.0 \mathrm{~Hz}, 3 \mathrm{H}) ;{ }^{13} \mathrm{C} \mathrm{NMR}\left(101 \mathrm{MHz}, \mathrm{CDCl}_{3}\right) \delta 141.6,140.3,136.1,132.6,128.6,128.1,127.9,127.37$, 127.35, 125.2, 24.4, 13.8; HRMS (ESI) $m / z$ calcd for $\mathrm{C}_{16} \mathrm{H}_{17} \mathrm{~S}[\mathrm{M}+\mathrm{H}]^{+}: 241.1045$ found 241.1043.

For $E$ isomer: ${ }^{1} \mathrm{H}$ NMR $\left(400 \mathrm{MHz}, \mathrm{CDCl}_{3}\right) \delta 7.34(\mathrm{~d}, J=8.4 \mathrm{~Hz}, 2 \mathrm{H}), 7.27-7.17(\mathrm{~m}, 7 \mathrm{H}), 6.13(\mathrm{t}, J=$ $7.6 \mathrm{~Hz}, 1 \mathrm{H}), 2.18-2.09(\mathrm{~m}, 2 \mathrm{H}), 0.98(\mathrm{t}, J=7.6 \mathrm{~Hz}, 3 \mathrm{H})$.<smiles>O=C(Sc1ccccc1)c1ccccc1</smiles>

$1 \mathrm{~A} 0.40 \mathrm{mmol}$

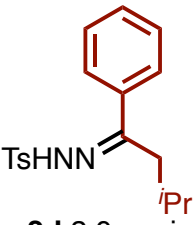

2d 2.0 equiv

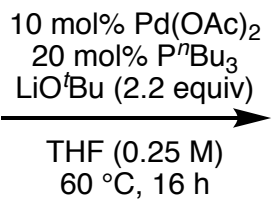

THF $(0.25 \mathrm{M})$
$60^{\circ} \mathrm{C}, 16 \mathrm{~h}$

(Z)-(3-Methyl-1-phenylbut-1-en-1-yl)(phenyl)sulfane (3Ad) ${ }^{[13]}$<smiles>CCC=C(Sc1ccccc1)c1ccccc1</smiles>

3Ad<smiles>CCCC(Sc1ccccc1)c1ccccc1</smiles>

4Ad 
Crude ${ }^{1} \mathrm{H}$ NMR analysis showed 3Ad:4Ad $=93: 7, Z: E=88: 12 ;{ }^{1} \mathrm{H}$ NMR peaks at $6.24 \mathrm{ppm}(\mathrm{q}$, $0.88 \mathrm{H})$ and $5.96 \mathrm{ppm}(\mathrm{q}, 0.12 \mathrm{H})$ were used. Purification by PTLC (hexane) afforded 3Ad as a colorless oil (55.4 mg, 54\% yield, as a mixture of diasteroisomers; $Z: E=88: 12$ ).

For $Z$ isomer: ${ }^{1} \mathrm{H}$ NMR $\left(400 \mathrm{MHz}, \mathrm{CDCl}_{3}\right) \delta 7.55(\mathrm{~d}, J=8.8 \mathrm{~Hz}, 2 \mathrm{H}), 7.24-7.08(\mathrm{~m}, 7 \mathrm{H}), 7.00(\mathrm{t}, J=$ $7.2 \mathrm{~Hz}, 1 \mathrm{H}), 6.24(\mathrm{~d}, J=9.6 \mathrm{~Hz}, 1 \mathrm{H}), 3.28-3.15(\mathrm{~m}, 1 \mathrm{H}), 1.09$ (d, $J=6.4 \mathrm{~Hz}, 6 \mathrm{H}) ;{ }^{13} \mathrm{C} \mathrm{NMR}(101$ $\left.\mathrm{MHz}, \mathrm{CDCl}_{3}\right) \delta 147.2,140.2,136.1,130.8,128.6,128.1,128.0,127.42,127.39,125.2,30.4,22.7$; HRMS (DART) $m / z$ calcd for $\mathrm{C}_{17} \mathrm{H}_{19} \mathrm{~S}[\mathrm{M}+\mathrm{H}]^{+}: 255.1202$ found 255.1199.

For $E$ isomer: ${ }^{1} \mathrm{H}$ NMR $\left(400 \mathrm{MHz}, \mathrm{CDCl}_{3}\right) \delta 7.32(\mathrm{~d}, J=8.8 \mathrm{~Hz}, 2 \mathrm{H}), 7.24-7.08(\mathrm{~m}, 8 \mathrm{H}), 5.96(\mathrm{~d}, J=$ $9.2 \mathrm{~Hz}, 1 \mathrm{H}), 2.59-2.47(\mathrm{~m}, 1 \mathrm{H}), 0.99(\mathrm{~d}, J=7.2 \mathrm{~Hz}, 6 \mathrm{H})$.<smiles>O=C(Sc1ccccc1)c1ccccc1</smiles>

$1 \mathrm{~A} 0.40 \mathrm{mmol}$

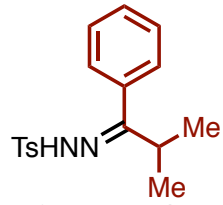

2e 2.0 equiv

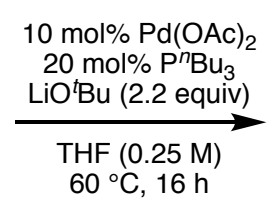
$60^{\circ} \mathrm{C}, 16 \mathrm{~h}$

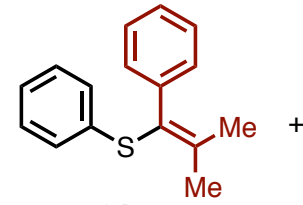

$3 \mathrm{Ae}$

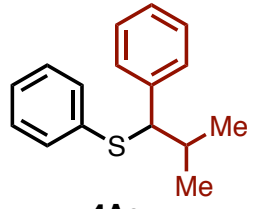

$4 \mathrm{Ae}$

\section{(2-Methyl-1-phenylprop-1-en-1-yl)(phenyl)sulfane (3Ae)}

Crude ${ }^{1} \mathrm{H}$ NMR analysis showed 3Ae:4Ae $=78: 22$. Purification by PTLC (hexane) afforded a mixture of $\mathbf{3 A e}$ and $4 \mathrm{Ae}$ as a colorless oil $(63.7 \mathrm{mg}$, 3Ae:4Ae $=78: 22)$. The yield of 3Ae was determined as $51 \%$. Further purification by PTLC (hexane) was performed to give pure 3Ae as a colorless oil for the characterization.

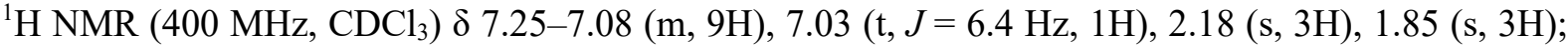
${ }^{13} \mathrm{C}$ NMR $\left(101 \mathrm{MHz}, \mathrm{CDCl}_{3}\right) \delta 141.8,140.9,136.4,129.6,128.7,128.5,127.6,127.0,126.6,125.4$, 23.5, 23.1; HRMS (ESI) $\mathrm{m} / z$ calcd for $\mathrm{C}_{16} \mathrm{H}_{17} \mathrm{~S}[\mathrm{M}+\mathrm{H}]^{+}: 241.1045$ found 241.1042.
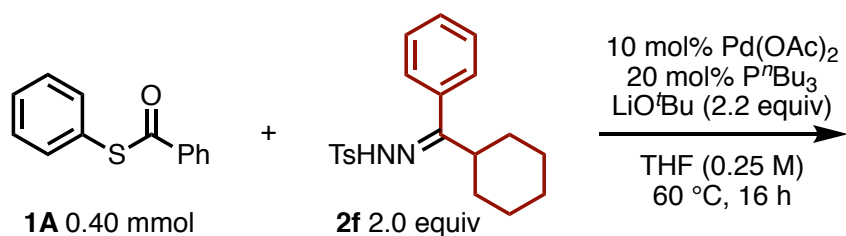

Cyclohexylidene(phenyl)methyl)(phenyl)sulfane (3Af) ${ }^{[14}$

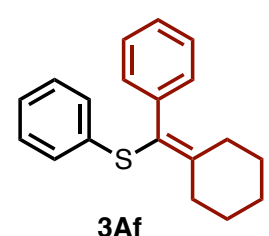

3Af

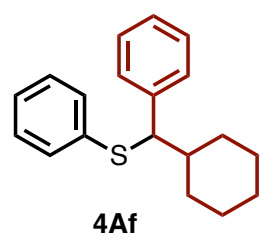

Crude ${ }^{1} \mathrm{H}$ NMR analysis showed 3Af:4Af $=74: 26$. Purification by PTLC (hexane) afforded a mixture of $\mathbf{3 A f}$ and 4Af as a colorless oil (86.3 $\mathrm{mg}$, 3Af:4Af $=74: 26)$. The yield of $\mathbf{3 A f}$ was determined as $57 \%$. Further purification by PTLC (hexane) was performed to give pure 3Af as a colorless oil for the characterization.

${ }^{1} \mathrm{H}$ NMR (400 MHz, $\left.\mathrm{CDCl}_{3}\right) \delta 7.41-7.08(\mathrm{~m}, 9 \mathrm{H}), 7.04(\mathrm{t}, J=7.2 \mathrm{~Hz}, 1 \mathrm{H}), 2.75(\mathrm{dd}, J=6.0,5.6 \mathrm{~Hz}$, $2 \mathrm{H}), 2.25(\mathrm{dd}, J=6.0,5.6 \mathrm{~Hz}, 2 \mathrm{H}), 1.72-1.56(\mathrm{~m}, 6 \mathrm{H}) ;{ }^{13} \mathrm{C} \mathrm{NMR}\left(101 \mathrm{MHz}, \mathrm{CDCl}_{3}\right) \delta 150.0,140.8$, 136.6, 129.6, 128.7, 128.5, 127.6, 126.6, 125.3, 124.2, 33.7, 33.2, 28.5, 28.4, 26.6; HRMS (DART) $m / z$ calcd for $\mathrm{C}_{19} \mathrm{H}_{21} \mathrm{~S}[\mathrm{M}+\mathrm{H}]^{+}: 281.1358$ found 281.1350. 


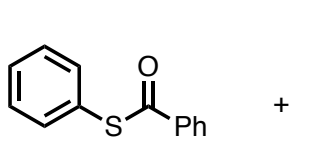

$1 \mathrm{~A} 0.40 \mathrm{mmo}$

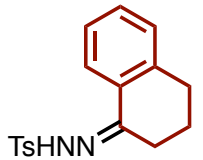

$2 \mathrm{~g} 2.0$ equiv

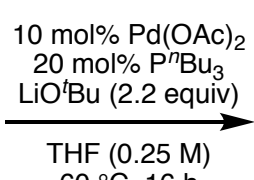

$60^{\circ} \mathrm{C}, 16 \mathrm{~h}$

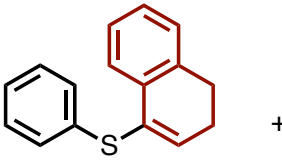

3Ag

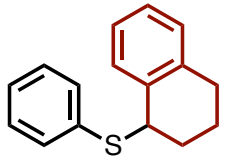

4Ag

(3,4-Dihydronaphthalen-1-yl)(phenyl)sulfane (3Ag) ${ }^{[15]}$

Crude ${ }^{1} \mathrm{H}$ NMR analysis showed 3Ag:4Ag $=92: 8$. Purification by PTLC (hexane/EtOAc $=97: 3$ ) afforded $3 \mathrm{Ag}$ as a colorless oil ( $65.5 \mathrm{mg}, 69 \%$ yield).

${ }^{1} \mathrm{H}$ NMR (400 MHz, $\left.\mathrm{CDCl}_{3}\right) \delta 7.56(\mathrm{~d}, J=6.8 \mathrm{~Hz}, 1 \mathrm{H}), 7.26-7.19(\mathrm{~m}, 4 \mathrm{H}), 7.15-7.09(\mathrm{~m}, 4 \mathrm{H}), 6.56(\mathrm{t}$, $J=5.2 \mathrm{~Hz}, 1 \mathrm{H}), 2.88(\mathrm{t}, J=8.0 \mathrm{~Hz}, 2 \mathrm{H}), 2.45(\mathrm{td}, J=8.0,5.2 \mathrm{~Hz}, 2 \mathrm{H}) ;{ }^{13} \mathrm{C} \mathrm{NMR}\left(101 \mathrm{MHz}, \mathrm{CDCl}_{3}\right) \delta$ 137.6, 136.4, 136.3, 133.0, 130.3, 128.8, 127.9, 127.6, 127.4, 126.7, 125.6, 125.1, 27.7, 24.6; HRMS (ESI) $m / z$ calcd for $\mathrm{C}_{16} \mathrm{H}_{15} \mathrm{~S}[\mathrm{M}+\mathrm{H}]^{+}: 239.0889$ found 239.0890 .<smiles>O=C(Sc1ccccc1)c1ccccc1</smiles>

$1 \mathrm{~A} 0.40 \mathrm{mmol}$

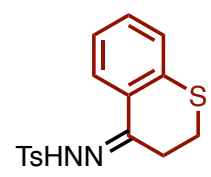

2h 2.0 equiv

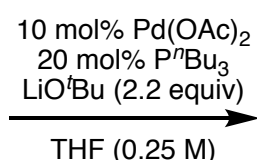

$60^{\circ} \mathrm{C}, 16 \mathrm{~h}$

\section{4-(Phenylthio)-2H-thiochromene (3Ah)}

Crude ${ }^{1} \mathrm{H}$ NMR analysis showed 3Ah:4Ah $=85: 15$. Purification by PTLC (hexane/EtOAc $=19: 1$ ) afforded $\mathbf{3 A h}$ as a colorless oil (51.7 $\mathrm{mg}, 50 \%$ yield).

${ }^{1} \mathrm{H}$ NMR (400 MHz, $\left.\mathrm{CDCl}_{3}\right) \delta 7.70(\mathrm{dd}, J=8.0,2.0 \mathrm{~Hz}, 1 \mathrm{H}), 7.29(\mathrm{dd}, J=7.2,2.0 \mathrm{~Hz}, 1 \mathrm{H}), 7.22-7.18$ (m, 4H), 7.15-7.06 (m, 2H), 7.02 (ddd, $J=8.0,7.2,1.6 \mathrm{~Hz}, 1 \mathrm{H}), 6.45$ (t, $J=5.6 \mathrm{~Hz}, 1 \mathrm{H}), 3.40$ (d, $J=$ $5.6 \mathrm{~Hz}, 2 \mathrm{H}) ;{ }^{13} \mathrm{C} \mathrm{NMR}\left(101 \mathrm{MHz}, \mathrm{CDCl}_{3}\right) \delta 135.5,133.4,133.1,131.9,129.0,128.6,128.2,127.5$, 127.4, 126.1, 125.8, 25.9 (one peak is missing due to overlapping.); HRMS (DART) $\mathrm{m} / z$ calcd for $\mathrm{C}_{15} \mathrm{H}_{13} \mathrm{~S}_{2}[\mathrm{M}+\mathrm{H}]^{+}: 257.0453$ found 257.0449.

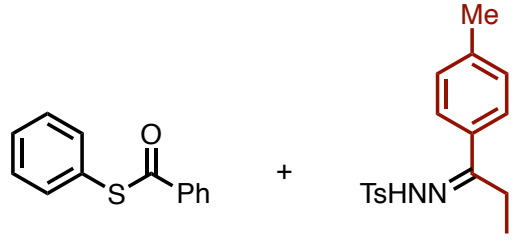

$1 \mathrm{~A} 0.40 \mathrm{mmol}$

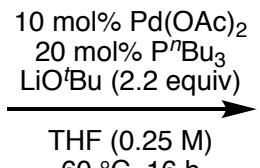

$60^{\circ} \mathrm{C}, 16 \mathrm{~h}$<smiles>C/C=C(/Sc1ccccc1)c1ccc(C)cc1</smiles>

3Ai

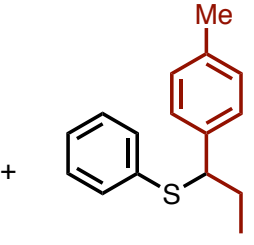

$4 \mathbf{A} \mathbf{i}$

(Z)-Phenyl(1-(p-tolyl)prop-1-en-1-yl)sulfane (3Ai) ${ }^{[11]}$

Crude ${ }^{1} \mathrm{H}$ NMR analysis showed 3Ai:4Ai $=93: 7, Z: E=88: 12 ;{ }^{1} \mathrm{H}$ NMR peaks at $6.47 \mathrm{ppm}(\mathrm{q}, 0.88 \mathrm{H})$ and $6.19 \mathrm{ppm}(\mathrm{q}, 0.12 \mathrm{H})$ were used. Purification by Isolera ${ }^{\circledR}$ (hexane) afforded 3Ai as a colorless oil (87.1 $\mathrm{mg}, 91 \%$ yield, as a mixture of diasteroisomers; $Z: E=88: 12)$.

For $Z$ isomer: ${ }^{1} \mathrm{H}$ NMR (400 MHz, $\left.\mathrm{CDCl}_{3}\right) \delta 7.44(\mathrm{~d}, J=8.4 \mathrm{~Hz}, 2 \mathrm{H}), 7.16-7.07(\mathrm{~m}, 4 \mathrm{H}), 7.05-6.96$ $(\mathrm{m}, 3 \mathrm{H}), 6.47$ (q, $J=6.8 \mathrm{~Hz}, 1 \mathrm{H}), 2.24(\mathrm{~s}, 3 \mathrm{H}), 2.05(\mathrm{~d}, J=6.8 \mathrm{~Hz}, 3 \mathrm{H}) ;{ }^{13} \mathrm{C} \mathrm{NMR}\left(101 \mathrm{MHz}, \mathrm{CDCl}_{3}\right)$ 
$\delta$ 137.7, 137.1, 136.2, 133.9, 133.8, 128.8, 128.6, 127.7, 127.1, 125.1, 21.0, 16.8; HRMS (DART) $\mathrm{m} / \mathrm{z}$ calcd for $\mathrm{C}_{16} \mathrm{H}_{17} \mathrm{~S}[\mathrm{M}+\mathrm{H}]^{+}: 241.1045$ found 241.1042 .

For $E$ isomer: ${ }^{1} \mathrm{H}$ NMR $\left(400 \mathrm{MHz}, \mathrm{CDCl}_{3}\right) \delta$ 7.27-7.23 (m, 4H), 7.16-7.07 (m, 2H), 7.05-6.96 (m, $3 \mathrm{H}), 6.19$ (q, $J=7.6 \mathrm{~Hz}, 1 \mathrm{H}), 2.25(\mathrm{~s}, 3 \mathrm{H}), 1.75(\mathrm{~d}, J=7.6 \mathrm{~Hz}, 3 \mathrm{H})$.

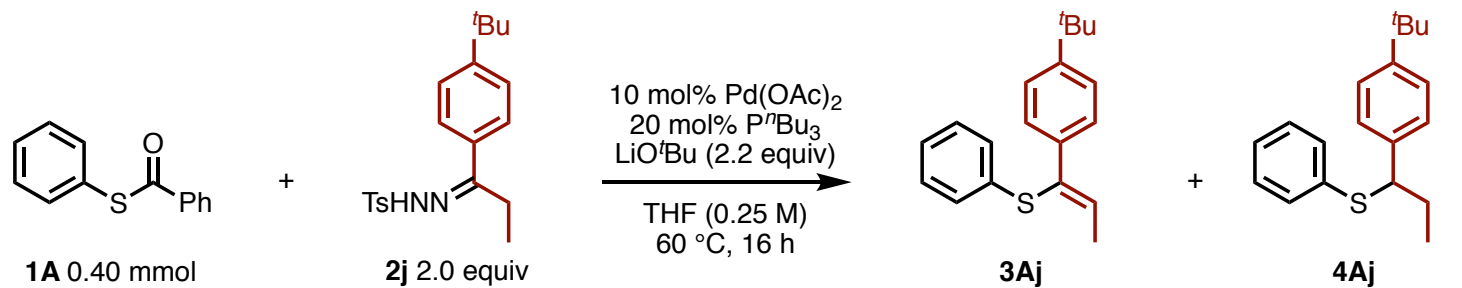

(Z)-(1-(4-(tert-Butyl)phenyl)prop-1-en-1-yl)(phenyl)sulfane (3Ajj)

Crude ${ }^{1} \mathrm{H}$ NMR analysis showed $\mathbf{3 A j}: 4 \mathbf{4} \mathbf{j}=90: 10, Z: E=93: 7 ;{ }^{1} \mathrm{H}$ NMR peaks at $6.55 \mathrm{ppm}(\mathrm{q}, 0.93 \mathrm{H})$ and $6.16 \mathrm{ppm}(\mathrm{q}, 0.07 \mathrm{H})$ were used. Purification by Isolera ${ }^{\circledR}$ (hexane) afforded $\mathbf{3 A j}$ as a white solid (101.2 $\mathrm{mg}, 90 \%$ yield, as a mixture of diasteroisomers; $Z: E=93: 7)$.

For $Z$ isomer: ${ }^{1} \mathrm{H}$ NMR $\left(400 \mathrm{MHz}, \mathrm{CDCl}_{3}\right) \delta 7.50(\mathrm{~d}, J=8.0 \mathrm{~Hz}, 2 \mathrm{H}), 7.25(\mathrm{~d}, J=8.0 \mathrm{~Hz}, 2 \mathrm{H}), 7.18-$ $7.10(\mathrm{~m}, 4 \mathrm{H}), 7.01$ (t, $J=8.0 \mathrm{~Hz} 1 \mathrm{H}), 6.55$ (q, $J=6.8 \mathrm{~Hz}, 1 \mathrm{H}), 2.04$ (d, $J=6.8 \mathrm{~Hz}, 3 \mathrm{H}), 1.26$ (s, 9H); ${ }^{13} \mathrm{C}$ NMR $\left(101 \mathrm{MHz}, \mathrm{CDCl}_{3}\right) \delta 150.4,137.8,136.5,134.6,133.4,128.7,127.2,126.8,125.1,125.0$, 34.4, 31.2, 16.9; HRMS (DART) $m / z$ calcd for $\mathrm{C}_{19} \mathrm{H}_{23} \mathrm{~S}[\mathrm{M}+\mathrm{H}]^{+}: 283.1515$ found 283.1511.

For $E$ isomer: ${ }^{1} \mathrm{H}$ NMR (400 MHz, $\left.\mathrm{CDCl}_{3}\right) \delta 7.30-7.23(\mathrm{~m}, 5 \mathrm{H}), 7.18-7.10(\mathrm{~m}, 4 \mathrm{H}), 6.16(\mathrm{q}, J=7.2$ $\mathrm{Hz}, 1 \mathrm{H}), 1.77$ (d, $J=7.2 \mathrm{~Hz}, 3 \mathrm{H}), 1.26$ (s, 9H).

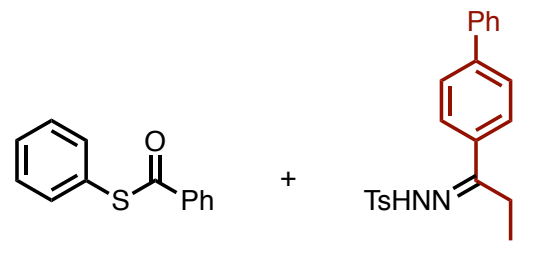

$1 \mathrm{~A} 0.20 \mathrm{mmol}$

2k 2.0 equiv

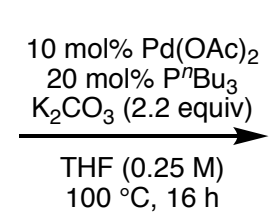

$100^{\circ} \mathrm{C}, 16 \mathrm{~h}$

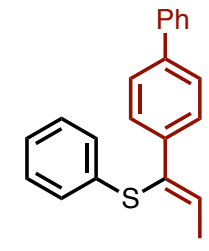

3Ak

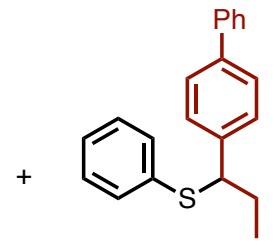

4Ak

\section{(Z)-(1-([1,1'-Biphenyl]-4-yl)prop-1-en-1-yl)(phenyl)sulfane (3Ak)}

The catalytic reaction was conducted by using $\mathrm{K}_{2} \mathrm{CO}_{3}$ instead of $\mathrm{LiO}^{t} \mathrm{Bu}$ at $100{ }^{\circ} \mathrm{C}(0.20 \mathrm{mmol}$ scale $)$. Crude ${ }^{1} \mathrm{H}$ NMR analysis showed 3Ak:4Ak $=92: 8, Z: E=95: 5 ;{ }^{1} \mathrm{H}$ NMR peaks at $6.60 \mathrm{ppm}(\mathrm{q}, 0.95 \mathrm{H})$ and $6.27 \mathrm{ppm}(\mathrm{q}, 0.05 \mathrm{H})$ were used. Purification by PTLC (hexane $/ \mathrm{CH}_{2} \mathrm{Cl}_{2}=19: 1$ ) afforded $\mathbf{3 A k}$ as a white solid (48.0 mg, 79\% yield, as a mixture of diasteroisomers; $Z: E=95: 5$ ).

For $Z$ isomer: ${ }^{1} \mathrm{H}$ NMR $\left(400 \mathrm{MHz}, \mathrm{CDCl}_{3}\right) \delta 7.63(\mathrm{~d}, J=8.0 \mathrm{~Hz}, 2 \mathrm{H}), 7.55(\mathrm{~d}, J=7.2 \mathrm{~Hz}, 2 \mathrm{H}), 7.47$ (d, $J=8.0 \mathrm{~Hz}, 2 \mathrm{H}), 7.40(\mathrm{dd}, J=8.0,7.2 \mathrm{~Hz}, 2 \mathrm{H}), 7.33-7.29(\mathrm{~m}, 1 \mathrm{H}), 7.21-7.13(\mathrm{~m}, 4 \mathrm{H}), 7.04(\mathrm{t}, J=7.2$ $\mathrm{Hz}, 1 \mathrm{H}), 6.60$ (q, $J=6.8 \mathrm{~Hz}, 1 \mathrm{H}), 2.11(\mathrm{~d}, J=6.8 \mathrm{~Hz}, 3 \mathrm{H}) ;{ }^{13} \mathrm{C} \mathrm{NMR}\left(101 \mathrm{MHz}, \mathrm{CDCl}_{3}\right) \delta 140.5$, $140.1,139.6,136.1,134.8,133.6,128.72,128.70,127.7,127.6,127.2,126.9,126.8,125.2,17.0$; HRMS (DART) $m / z$ calcd for $\mathrm{C}_{21} \mathrm{H}_{19} \mathrm{~S}[\mathrm{M}+\mathrm{H}]^{+}: 303.1202$ found 303.1196. 
For $E$ isomer: ${ }^{1} \mathrm{H}$ NMR $\left(400 \mathrm{MHz}, \mathrm{CDCl}_{3}\right) \delta 7.59-7.58(\mathrm{~m}, 2 \mathrm{H}), 7.51-7.50(\mathrm{~m}, 2 \mathrm{H}), 7.44-7.43(\mathrm{~m}$, 2H), 7.33-7.29 (m, 7H), 6.98 (m, 1H), 6.27 (q, $J=7.2 \mathrm{~Hz}, 1 \mathrm{H}), 1.83$ (d, $J=7.2 \mathrm{~Hz}, 3 \mathrm{H})$.

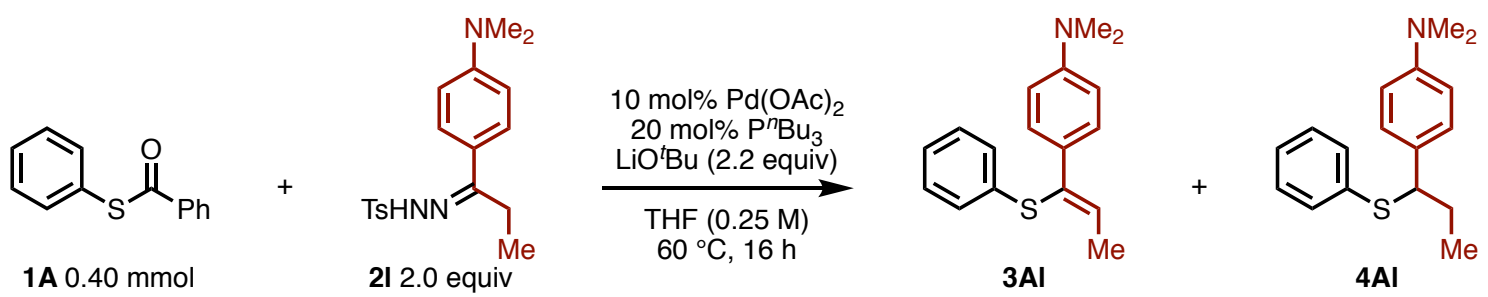

(Z)-N,N-Dimethyl-4-(1-(phenylthio)prop-1-en-1-yl)aniline (3AI)

Crude ${ }^{1} \mathrm{H}$ NMR analysis showed 3Al:4Al = 77:23, $Z: E=93: 7 ;{ }^{1} \mathrm{H}$ NMR peaks at $6.42 \mathrm{ppm}(\mathrm{q}, 0.89 \mathrm{H})$ and $6.13 \mathrm{ppm}(\mathrm{q}, 0.11 \mathrm{H})$ were used. Purification by PTLC (hexane/EtOAc $=19: 1)$ afforded a mixture of $\mathbf{3 A l}$ and $\mathbf{4 A l}$ as a white solid $(81.5 \mathrm{mg}$, , 3Al:4Al $=81: 19, Z: E=89: 11)$. The yield of $\mathbf{3 A l}$ was determined as $61 \%$. Further purification by PTLC (hexane/EtOA $=19: 1$ ) was performed to give pure 3Al as a white solid for the characterization.

For $Z$ isomer: ${ }^{1} \mathrm{H}$ NMR (400 MHz, $\left.\mathrm{CDCl}_{3}\right) \delta 7.45(\mathrm{~d}, J=8.8 \mathrm{~Hz}, 2 \mathrm{H}), 7.20-7.11(\mathrm{~m}, 4 \mathrm{H}), 7.04-6.99$ $(\mathrm{m}, 1 \mathrm{H}), 6.60(\mathrm{~d}, J=8.8 \mathrm{~Hz}, 2 \mathrm{H}), 6.42(\mathrm{q}, J=6.8 \mathrm{~Hz}, 1 \mathrm{H}), 2.91(\mathrm{~s}, 6 \mathrm{H}), 2.04(\mathrm{~d}, J=6.8 \mathrm{~Hz}, 3 \mathrm{H}) ;{ }^{13} \mathrm{C}$ NMR (101 MHz, $\left.\mathrm{CDCl}_{3}\right) \delta 149.9,136.8,133.4,131.4,128.7,128.6,128.0,127.4,124.8,112.0,40.4$, 16.8; HRMS (ESI) $m / z$ calcd for $\mathrm{C}_{17} \mathrm{H}_{20} \mathrm{NS}[\mathrm{M}+\mathrm{H}]^{+}: 270.1311$ found 270.1309.

For $E$ isomer: ${ }^{1} \mathrm{H}$ NMR $\left(400 \mathrm{MHz}, \mathrm{CDCl}_{3}\right) \delta 7.29(\mathrm{~d}, J=8.4 \mathrm{~Hz}, 2 \mathrm{H}), 7.25-7.20(\mathrm{~m}, 2 \mathrm{H}), 7.09-7.06$ $(\mathrm{m}, 2 \mathrm{H}), 6.97-6.93(\mathrm{~m}, 1 \mathrm{H}), 6.63-6.58(\mathrm{~m}, 2 \mathrm{H}), 6.13$ (q, $J=7.2 \mathrm{~Hz}, 1 \mathrm{H}), 2.93(\mathrm{~s}, 6 \mathrm{H}), 1.81$ (d, $J=7.2$ $\mathrm{Hz}, 3 \mathrm{H})$.

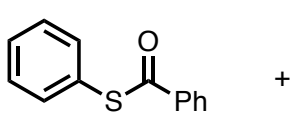

$1 \mathrm{~A} 0.40 \mathrm{mmol}$

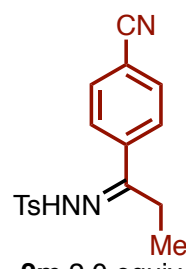

$2 \mathrm{~m} 2.0$ equiv

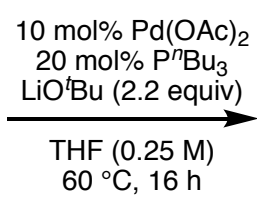

$60^{\circ} \mathrm{C}, 16 \mathrm{~h}$<smiles>Cc1ccc(/C(=C/O)Sc2ccccc2)cc1</smiles>

3Am

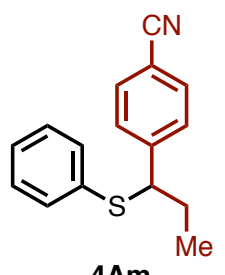

4Am

(Z)-4-(1-(Phenylthio)prop-1-en-1-yl)benzonitrile (3Am)

Crude ${ }^{1} \mathrm{H}$ NMR analysis showed 3Am:4Am $=80: 20, Z: E=93: 7 ;{ }^{1} \mathrm{H}$ NMR peaks at $6.60 \mathrm{ppm}(\mathrm{q}$, $0.93 \mathrm{H})$ and $6.49 \mathrm{ppm}(\mathrm{q}, 0.07 \mathrm{H})$ were used. Purification by Isolera ${ }^{\circledR}$ (hexane/EtOAc $=19: 1$ to 9:1) afforded an inseparable mixture of $\mathbf{3 A m}$ and $\mathbf{4 A m}$ as a colorless oil $(89.1 \mathrm{mg}, \mathbf{3 A m}: \mathbf{4 A m}=80: 20)$. The yield of $\mathbf{3 A m}$ was determined as $71 \%$.

For $Z$ isomer: ${ }^{1} \mathrm{H}$ NMR (400 MHz, $\left.\mathrm{CDCl}_{3}\right) \delta 7.63(\mathrm{~d}, J=8.0 \mathrm{~Hz}, 2 \mathrm{H}), 7.51(\mathrm{~d}, J=8.0 \mathrm{~Hz}, 2 \mathrm{H}), 7.20$ $7.10(\mathrm{~m}, 4 \mathrm{H}), 7.07$ (t, $J=6.8 \mathrm{~Hz}, 1 \mathrm{H}), 6.60(\mathrm{q}, J=6.8 \mathrm{~Hz}, 1 \mathrm{H}), 2.14(\mathrm{~d}, J=6.8 \mathrm{~Hz}, 3 \mathrm{H}) ;{ }^{13} \mathrm{C} \mathrm{NMR}$ $\left(101 \mathrm{MHz}, \mathrm{CDCl}_{3}\right) \delta 145.1,137.3,134.8,133.4,132.0,128.9,128.3,127.9,125.9,118.8,110.8,17.1$; HRMS (ESI) $m / z$ calcd for $\mathrm{C}_{16} \mathrm{H}_{13} \mathrm{NS}[\mathrm{M}]^{+}: 251.0763$ found 251.0765 . 


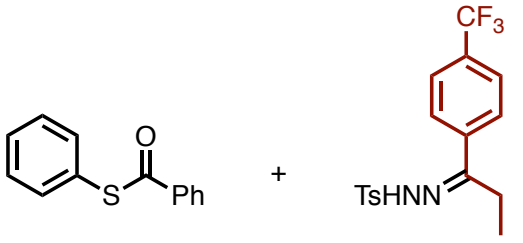

$1 \mathrm{~A} 0.40 \mathrm{mmol}$

2n 2.0 equiv

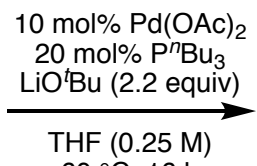

$60^{\circ} \mathrm{C}, 16 \mathrm{~h}$<smiles>C/C=C(\Sc1ccccc1)c1ccc(C(F)(F)F)cc1</smiles>

3An<smiles>CCC(Sc1ccccc1)c1ccc(C(F)(F)F)cc1</smiles>

4An

\section{(Z)-Phenyl(1-(4-(trifluoromethyl)phenyl)prop-1-en-1-yl)sulfane (3An)}

Crude ${ }^{1} \mathrm{H}$ NMR analysis showed 3An:4An $=90: 10, Z: E=97: 3 ;{ }^{1} \mathrm{H}$ NMR peaks at $6.57 \mathrm{ppm}(\mathrm{q}$, $0.97 \mathrm{H})$ and $6.33 \mathrm{ppm}(\mathrm{q}, 0.03 \mathrm{H})$ were used. Purification by Isolera ${ }^{\circledR}$ (hexane) afforded 3An as a colorless oil (102.1 mg, 87\% yield, as a mixture of diasteroisomers; $Z: E=97: 3)$.

For $Z$ isomer: ${ }^{1} \mathrm{H}$ NMR (400 MHz, $\left.\mathrm{CDCl}_{3}\right) \delta 7.64(\mathrm{~d}, J=8.4 \mathrm{~Hz}, 2 \mathrm{H}), 7.46(\mathrm{~d}, J=8.4 \mathrm{~Hz}, 2 \mathrm{H}), 7.18-$ $7.11(\mathrm{~m}, 4 \mathrm{H}), 7.08-7.01(\mathrm{~m}, 1 \mathrm{H}), 6.57(\mathrm{q}, J=6.8 \mathrm{~Hz}, 1 \mathrm{H}), 2.11(\mathrm{~d}, J=6.8 \mathrm{~Hz}, 3 \mathrm{H}) ;{ }^{13} \mathrm{C}$ NMR $(101$ $\left.\mathrm{MHz}, \mathrm{CDCl}_{3}\right) \delta 144.1,136.7,135.2,133.4,129.3\left(\mathrm{q}, J_{\mathrm{C}-\mathrm{F}}=32.9 \mathrm{~Hz}\right), 128.8,128.1,127.5,125.7,125.1$ $\left(\mathrm{q}, J_{\mathrm{C}-\mathrm{F}}=3.8 \mathrm{~Hz}\right), 124.1\left(\mathrm{q}, J_{\mathrm{C}-\mathrm{F}}=275.7 \mathrm{~Hz}\right), 17.0$; HRMS (DART) $\mathrm{m} / z$ calcd for $\mathrm{C}_{16} \mathrm{H}_{14} \mathrm{~F}_{3} \mathrm{~S}[\mathrm{M}+\mathrm{H}]^{+}$: 295.0763 found 295.0757.

For $E$ isomer: ${ }^{1} \mathrm{H}$ NMR (400 MHz, $\left.\mathrm{CDCl}_{3}\right) \delta 7.46(\mathrm{~d}, J=8.4 \mathrm{~Hz}, 2 \mathrm{H}), 7.35-7.23(\mathrm{~m}, 7 \mathrm{H}), 6.33(\mathrm{q}, J=$ $7.2 \mathrm{~Hz}, 1 \mathrm{H}), 1.76(\mathrm{~d}, J=7.2 \mathrm{~Hz}, 3 \mathrm{H})$.

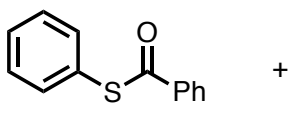

$1 \mathrm{~A} 0.40 \mathrm{mmol}$<smiles>CCC(=N)c1ccc(Br)cc1</smiles>

202.0 equiv

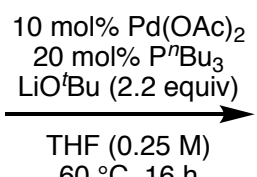

$60^{\circ} \mathrm{C}, 16 \mathrm{~h}$<smiles>C/C=C(/Sc1ccccc1)c1ccc(Br)cc1</smiles>

3AO

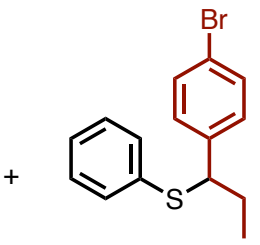

4Ao

\section{(Z)-(1-(4-Bromophenyl)prop-1-en-1-yl)(phenyl)sulfane (3Ao)}

Crude ${ }^{1} \mathrm{H}$ NMR analysis showed 3Ao:4Ao $=95: 5, Z: E=87: 13 ;{ }^{1} \mathrm{H}$ NMR peaks at $6.48 \mathrm{ppm}(\mathrm{q}, 0.87 \mathrm{H})$ and $6.26 \mathrm{ppm}(\mathrm{q}, 0.13 \mathrm{H})$ were used. Purification by Isolera ${ }^{\circledR}$ (hexane) afforded $\mathbf{3 A o}$ as a colorless oil (108.0 mg, 88\% yield, as a mixture of diasteroisomers; $Z: E=95: 5)$.

For $Z$ isomer: ${ }^{1} \mathrm{H}$ NMR $\left(400 \mathrm{MHz}, \mathrm{CDCl}_{3}\right) \delta 7.37(\mathrm{~d}, J=8.8 \mathrm{~Hz}, 2 \mathrm{H}), 7.33(\mathrm{~d}, J=8.8 \mathrm{~Hz}, 2 \mathrm{H}), 7.16-$ $7.11(\mathrm{~m}, 4 \mathrm{H}), 7.09-7.01(\mathrm{~m}, 1 \mathrm{H}), 6.48(\mathrm{q}, J=6.8 \mathrm{~Hz}, 1 \mathrm{H}), 2.08(\mathrm{~d}, J=6.8 \mathrm{~Hz}, 3 \mathrm{H}) ;{ }^{13} \mathrm{C}$ NMR $(101$ $\left.\mathrm{MHz}, \mathrm{CDCl}_{3}\right) \delta 139.5,135.4,135.0,133.4,131.2,128.9,128.8,128.1,125.5,121.3,16.9$; HRMS (DART) $\mathrm{m} / z$ calcd for $\mathrm{C}_{15} \mathrm{H}_{14} \mathrm{BrS}[\mathrm{M}+\mathrm{H}]^{+}: 304.9994$ found 304.9988 .

For $E$ isomer: ${ }^{1} \mathrm{H}$ NMR (400 MHz, $\left.\mathrm{CDCl}_{3}\right) \delta 7.16-7.11(\mathrm{~m}, 9 \mathrm{H}), 6.26(\mathrm{q}, J=7.2 \mathrm{~Hz}, 1 \mathrm{H}), 1.75(\mathrm{~d}, J=$ $7.2 \mathrm{~Hz}, 3 \mathrm{H})$ 


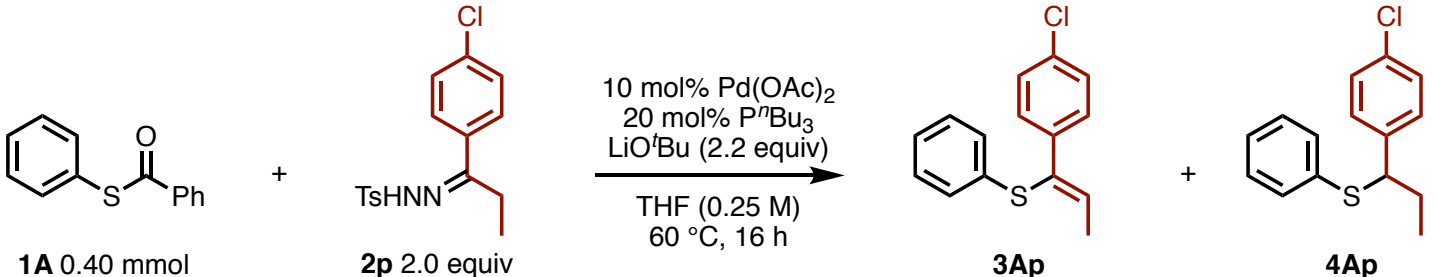

\section{4-((2,4-Dimethylphenyl)thio)pyridine (3Ap)}

Crude ${ }^{1} \mathrm{H}$ NMR analysis showed 3Ap:4Ap $=94: 6, Z: E=95: 5 ;{ }^{1} \mathrm{H}$ NMR peaks at $6.47 \mathrm{ppm}(\mathrm{q}, 0.95 \mathrm{H})$ and $6.26 \mathrm{ppm}(\mathrm{q}, 0.05 \mathrm{H})$ were used. Purification by Isolera ${ }^{\circledR}$ (hexane) afforded $\mathbf{3 A p}$ as a colorless oil (90.8 mg, $87 \%$ yield, as a mixture of diasteroisomers; $Z: E=95: 5)$.

For $Z$ isomer: ${ }^{1} \mathrm{H}$ NMR (400 MHz, $\left.\mathrm{CDCl}_{3}\right) \delta 7.46(\mathrm{~d}, J=8.8 \mathrm{~Hz}, 2 \mathrm{H}), 7.17(\mathrm{~d}, J=8.8 \mathrm{~Hz}, 2 \mathrm{H}), 7.14$ $7.10(\mathrm{~m}, 4 \mathrm{H}), 7.06-7.01(\mathrm{~m}, 1 \mathrm{H}), 6.47(\mathrm{q}, J=6.8 \mathrm{~Hz}, 1 \mathrm{H}), 2.08(\mathrm{~d}, J=6.8 \mathrm{~Hz}, 3 \mathrm{H}) ;{ }^{13} \mathrm{C} \mathrm{NMR}(101$ $\left.\mathrm{MHz}, \mathrm{CDCl}_{3}\right) \delta 139.0,135.4,134.9,133.3,133.1,128.7,128.6,128.3,128.0,125.5,16.9 ;$ HRMS (DART) $\mathrm{m} / z$ calcd for $\mathrm{C}_{15} \mathrm{H}_{14} \mathrm{ClS}[\mathrm{M}+\mathrm{H}]^{+}: 261.0499$ found 261.0494.

For $E$ isomer: ${ }^{1} \mathrm{H}$ NMR $\left(400 \mathrm{MHz}, \mathrm{CDCl}_{3}\right) \delta 7.30-7.27(\mathrm{~m}, 2 \mathrm{H}), 7.14-7.10(\mathrm{~m}, 7 \mathrm{H}), 6.26(\mathrm{q}, J=7.2$ $\mathrm{Hz}, 1 \mathrm{H}), 1.75(\mathrm{~d}, J=7.2 \mathrm{~Hz}, 3 \mathrm{H})$.

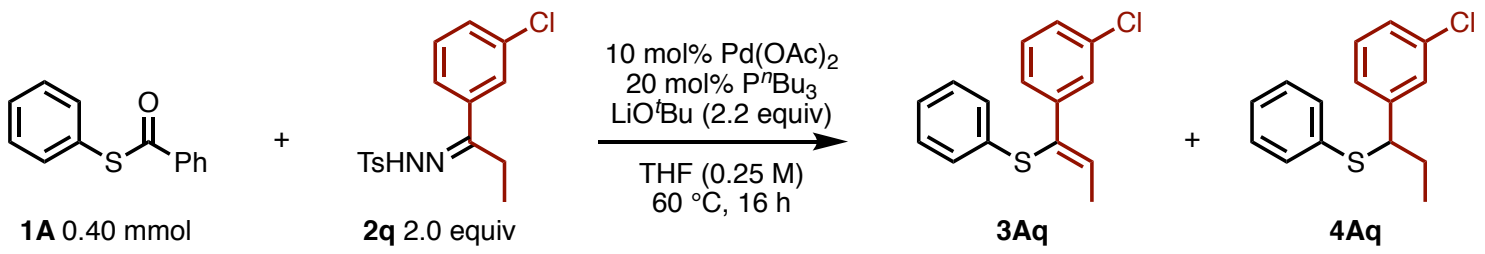

\section{(Z)-(1-(3-Chlorophenyl)prop-1-en-1-yl)(phenyl)sulfane (3Aq)}

Crude ${ }^{1} \mathrm{H}$ NMR analysis showed 3Aq:4Aq $=85: 15, Z: E=89: 11 ;{ }^{1} \mathrm{H}$ NMR peaks at $6.50 \mathrm{ppm}(\mathrm{q}$, $0.89 \mathrm{H})$ and $6.25 \mathrm{ppm}(\mathrm{q}, 0.11 \mathrm{H})$ were used. Purification by Isolera ${ }^{\circledR}$ (hexane) afforded 3Aq as a colorless oil (82.9 mg, 79\% yield, as a mixture of diasteroisomers; $Z: E=96: 4)$.

For $Z$ isomer: ${ }^{1} \mathrm{H}$ NMR $\left(400 \mathrm{MHz}, \mathrm{CDCl}_{3}\right) \delta 7.53(\mathrm{~s}, 1 \mathrm{H}), 7.43-7.40(\mathrm{~m}, 1 \mathrm{H}), 7.15-7.11(\mathrm{~m}, 6 \mathrm{H})$, 7.07-7.01 (m, 1H), $6.50(\mathrm{q}, J=6.8 \mathrm{~Hz}, 1 \mathrm{H}), 2.08(\mathrm{~d}, J=6.8 \mathrm{~Hz}, 3 \mathrm{H}) ;{ }^{13} \mathrm{C}$ NMR $\left(101 \mathrm{MHz}, \mathrm{CDCl}_{3}\right) \delta$ $142.5,135.7,135.4,134.0,133.1,129.3,128.8,128.0,127.4,127.3,125.54,125.51,16.9$; HRMS (DART) $m / z$ calcd for $\mathrm{C}_{15} \mathrm{H}_{14} \mathrm{ClS}[\mathrm{M}+\mathrm{H}]^{+}: 261.0499$ found 261.0497.

For $E$ isomer: ${ }^{1} \mathrm{H}$ NMR $\left(400 \mathrm{MHz}, \mathrm{CDCl}_{3}\right) \delta$ 7.35-7.32 (m, 2H), 7.24-7.20 (m, 6H), 6.96-6.89 (m, $1 \mathrm{H}), 6.25$ (q, $J=7.2 \mathrm{~Hz}, 1 \mathrm{H}), 1.75(\mathrm{~d}, J=7.6 \mathrm{~Hz}, 3 \mathrm{H})$.

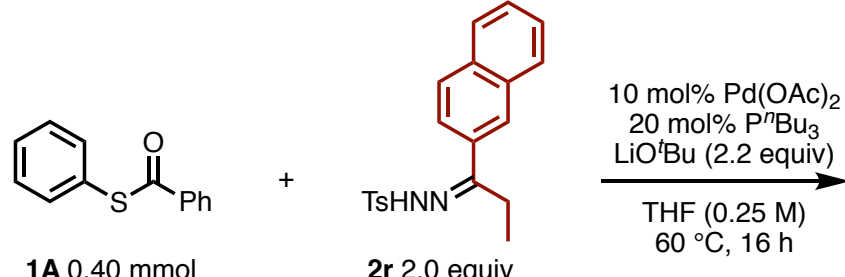

$1 \mathrm{~A} 0.40 \mathrm{mmol}$

$$
\text { 2r } 2.0 \text { equiv }
$$<smiles>C/C=C(\Sc1ccccc1)c1ccc2ccccc2c1</smiles>

$3 \mathrm{Ar}$<smiles>CCC(Sc1ccccc1)c1ccc2ccccc2c1</smiles>

4Ar

(Z)-(1-(Naphthalen-2-yl)prop-1-en-1-yl)(phenyl)sulfane (3Ar) 
Crude ${ }^{1} \mathrm{H}$ NMR analysis showed 3Ar:4Ar $=93: 7, Z: E=95: 5 ;{ }^{1} \mathrm{H}$ NMR peaks at $6.62 \mathrm{ppm}(\mathrm{q}, 0.95 \mathrm{H})$ and $6.32 \mathrm{ppm}(\mathrm{q}, 0.05 \mathrm{H})$ were used. Purification by PTLC (hexane $/ \mathrm{CH}_{2} \mathrm{Cl}_{2}=19: 1$ ) afforded $3 \mathrm{Ar}$ as a white solid (93.1 mg, 84\% yield, as a mixture of diasteroisomers; $Z: E=95: 5)$.

For $Z$ isomer: ${ }^{1} \mathrm{H}$ NMR (400 MHz, $\left.\mathrm{CDCl}_{3}\right) \delta 8.02(\mathrm{~s}, 1 \mathrm{H}), 7.75-7.65(\mathrm{~m}, 4 \mathrm{H}), 7.40-7.34(\mathrm{~m}, 2 \mathrm{H}), 7.19$ (d, $J=8.4 \mathrm{~Hz}, 2 \mathrm{H}), 7.08(\mathrm{dd}, J=8.4,7.2 \mathrm{~Hz}, 2 \mathrm{H}), 6.96(\mathrm{t}, J=7.2 \mathrm{~Hz}, 1 \mathrm{H}), 6.62(\mathrm{q}, J=6.8 \mathrm{~Hz}, 1 \mathrm{H})$, $2.11(\mathrm{~d}, J=6.8 \mathrm{~Hz}, 3 \mathrm{H}) ;{ }^{13} \mathrm{C} \mathrm{NMR}\left(101 \mathrm{MHz}, \mathrm{CDCl}_{3}\right) \delta 138.0,135.9,135.3,134.1,133.2,132.7$, 128.7, 128.1 127.8, 127.7, 127.4, 126.3, 126.0, 125.8, 125.33, 125.25, 17.0; HRMS (DART) $\mathrm{m} / z$ calcd for $\mathrm{C}_{19} \mathrm{H}_{17} \mathrm{~S}[\mathrm{M}+\mathrm{H}]^{+}: 277.1045$ found 277.1040.

For $E$ isomer: ${ }^{1} \mathrm{H}$ NMR $\left(400 \mathrm{MHz}, \mathrm{CDCl}_{3}\right) \delta 7.86-7.79(\mathrm{~m}, 4 \mathrm{H}), 7.75-7.65(\mathrm{~m}, 4 \mathrm{H}), 7.40-7.34(\mathrm{~m}$, 4H), $6.32(\mathrm{q}, J=7.2 \mathrm{~Hz}, 1 \mathrm{H}), 1.79(\mathrm{~d}, J=7.2 \mathrm{~Hz}, 3 \mathrm{H})$.

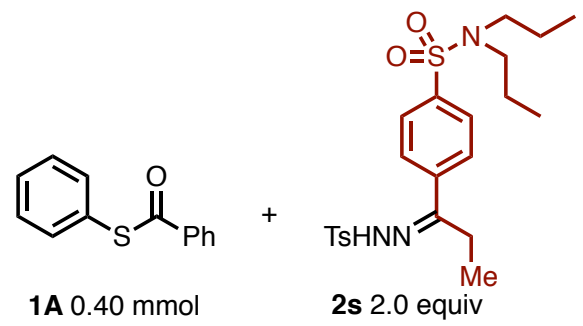

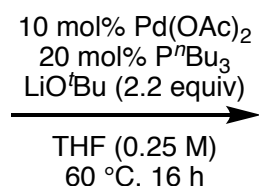

$60^{\circ} \mathrm{C}, 16 \mathrm{~h}$

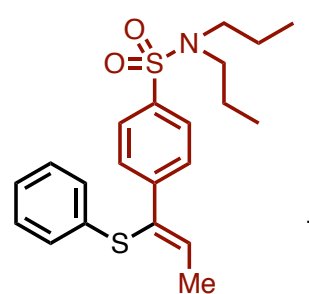

3As

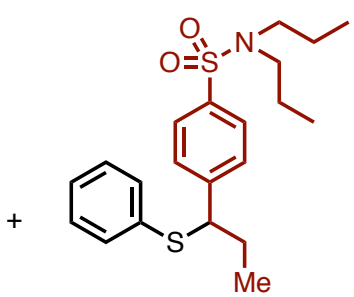

4As

\section{(Z)-4-(1-(Phenylthio)prop-1-en-1-yl)- $N, N$-dipropylbenzenesulfonamide (3As)}

Crude ${ }^{1} \mathrm{H}$ NMR analysis showed 3As:4As $=87: 13, Z: E=92: 8 ;{ }^{1} \mathrm{H}$ NMR peaks at $6.57 \mathrm{ppm}(\mathrm{q}, 0.92 \mathrm{H})$ and $6.34 \mathrm{ppm}(\mathrm{q}, 0.08 \mathrm{H})$ were used. Purification by Isolera ${ }^{\circledR}$ (hexane/EtOAc $=19: 1$ to 9:1 afforded an inseparable mixture of 3As and 4As as a white solid (147.2 $\mathrm{mg}, Z: E=96: 4, \mathbf{3 A s}: \mathbf{4 A s}=84: 16)$. The yield of 3As was determined as $79 \%$.

For $Z$ isomer: ${ }^{1} \mathrm{H}$ NMR $\left(400 \mathrm{MHz}, \mathrm{CDCl}_{3}\right) \delta$ 7.65-7.61 (m, 4H), 7.16-7.09 (m, 4H), 7.06-7.00 (m, $1 \mathrm{H}), 6.57(\mathrm{q}, J=6.8 \mathrm{~Hz}, 1 \mathrm{H}), 3.01(\mathrm{t}, J=8.0 \mathrm{~Hz}, 4 \mathrm{H}), 2.12$ (d, $J=6.8 \mathrm{~Hz}, 3 \mathrm{H}), 1.52-1.42(\mathrm{~m}, 4 \mathrm{H})$, $0.81(\mathrm{t}, J=7.2 \mathrm{~Hz}, 6 \mathrm{H}) ;{ }^{13} \mathrm{C} \mathrm{NMR}\left(101 \mathrm{MHz}, \mathrm{CDCl}_{3}\right) \delta 144.3,138.5,136.5,134.8,133.3,128.6$, 128.2, 127.7, 126.8, 125.6, 49.6, 21.7, 16.9, 11.0; HRMS (DART) $m / z$ calcd for $\mathrm{C}_{21} \mathrm{H}_{28} \mathrm{NO}_{2} \mathrm{~S}_{2}[\mathrm{M}+\mathrm{H}]^{+}$: 390.1556 found 390.1549 .

For $E$ isomer: ${ }^{1} \mathrm{H}$ NMR $\left(400 \mathrm{MHz}, \mathrm{CDCl}_{3}\right) \delta 7.66(\mathrm{~d}, J=8.4 \mathrm{~Hz}, 2 \mathrm{H}), 7.45(\mathrm{~d}, J=8.4 \mathrm{~Hz}, 2 \mathrm{H}), 7.22-$ 7.20 (m, 2H), 7.16-7.09 (m, 5H), 6.34 (q, $J=7.6 \mathrm{~Hz}, 1 \mathrm{H}), 3.01$ (t, $J=8.0 \mathrm{~Hz}, 4 \mathrm{H}), 1.77$ (d, $J=7.6 \mathrm{~Hz}$, $3 \mathrm{H}), 1.52-1.42(\mathrm{~m}, 4 \mathrm{H}), 0.84(\mathrm{t}, J=7.2 \mathrm{~Hz}, 6 \mathrm{H})$.

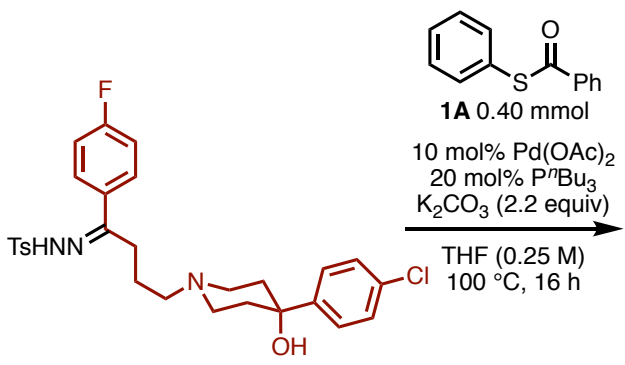

62.0 equiv

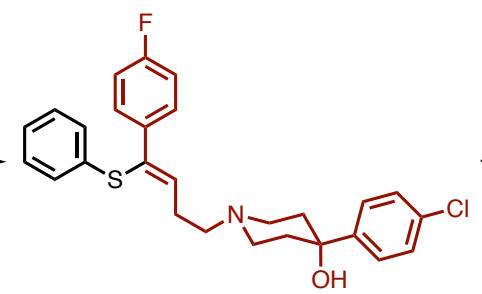

7

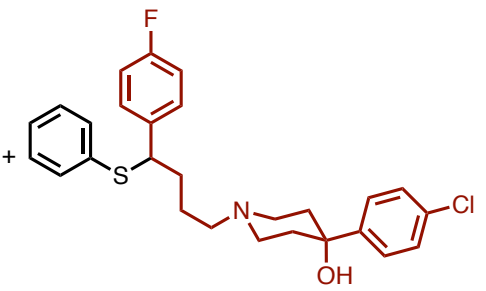

7 


\section{(Z)-4-(4-Chlorophenyl)-1-(4-(4-fluorophenyl)-4-(phenylthio)but-3-en-1-yl)piperidin-4-ol (7)}

The catalytic reaction was conducted by using $\mathrm{K}_{2} \mathrm{CO}_{3}$ instead of $\mathrm{LiO}^{t} \mathrm{Bu}$ at $100{ }^{\circ} \mathrm{C}(84.6 \mu \mathrm{mol}$ scale). Crude ${ }^{1} \mathrm{H}$ NMR analysis showed 7:7' $=77: 23, Z: E=90: 10 ;{ }^{1} \mathrm{H}$ NMR peaks at $6.37 \mathrm{ppm}(\mathrm{q}, 0.90 \mathrm{H})$ and $6.15 \mathrm{ppm}(\mathrm{q}, 0.10 \mathrm{H})$ were used. Purification by PTLC (hexane/acetone $=2: 1$ ) afforded 7 as a white solid (16.8 mg, $42 \%$ yield).

For $Z$ isomer: ${ }^{1} \mathrm{H}$ NMR (400 MHz, $\left.\mathrm{CDCl}_{3}\right) \delta 7.51(\mathrm{dd}, J=8.8,5.6 \mathrm{~Hz}, 2 \mathrm{H}), 7.44(\mathrm{~d}, J=8.8 \mathrm{~Hz}, 2 \mathrm{H})$, $7.32(\mathrm{~d}, J=8.8 \mathrm{~Hz}, 2 \mathrm{H}), 7.21-7.10(\mathrm{~m}, 3 \mathrm{H}), 7.08-7.02(\mathrm{~m}, 1 \mathrm{H}), 6.91(\mathrm{t}, J=8.8 \mathrm{~Hz}, 2 \mathrm{H}), 6.37(\mathrm{t}, J=$ $6.8 \mathrm{~Hz}, 1 \mathrm{H}), 2.87(\mathrm{ddd}, J=11.6,4.4,2.0 \mathrm{~Hz}, 2 \mathrm{H}), 2.78(\mathrm{td}, J=7.6,6.8 \mathrm{~Hz}, 2 \mathrm{H}), 2.61(\mathrm{t}, J=7.6 \mathrm{~Hz}$, 2H), 2.50 (ddd, $J=12.0,11.6,2.0 \mathrm{~Hz}, 2 \mathrm{H}), 2.13$ (ddd, $J=12.0,11.6,4.4 \mathrm{~Hz}, 2 \mathrm{H}$ ), 1.73 (ddd, $J=11.4$, 2.8, $2.0 \mathrm{~Hz}, 2 \mathrm{H}) ;{ }^{13} \mathrm{C}$ NMR $\left(101 \mathrm{MHz}, \mathrm{CDCl}_{3}\right) \delta 162.2\left(\mathrm{~d}, J_{\mathrm{C}-\mathrm{F}}=249.7 \mathrm{~Hz}\right), 146.8,136.8,136.2\left(\mathrm{~d}, J_{\mathrm{C}-}\right.$ $\mathrm{F}=2.9 \mathrm{~Hz}), 135.4,133.8,132.8,129.1\left(\mathrm{~d}, J_{\mathrm{C}-\mathrm{F}}=8.7 \mathrm{~Hz}\right), 128.7,128.4,126.1,125.6,115.0\left(\mathrm{~d}, J_{\mathrm{C}-\mathrm{F}}=\right.$ $22.2 \mathrm{~Hz}$ ), 71.1, 57.7, 49.2, 38.4, 29.7, 28.7; HRMS (DART) $\mathrm{m} / \mathrm{z}$ calcd for $\mathrm{C}_{27} \mathrm{H}_{28} \mathrm{ClFNOS}[\mathrm{M}+\mathrm{H}]^{+}$: 468.1559 found 468.1556 .

\section{One-Pot Operation}
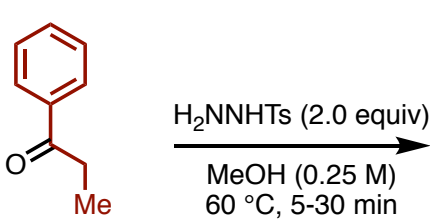

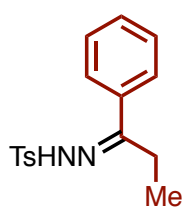

$2 a$

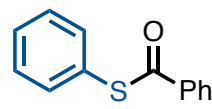

$1 \mathrm{~A}$ (1.0 equiv)

$10 \mathrm{~mol} \% \mathrm{Pd}(\mathrm{OAc})_{2}$ $20 \mathrm{~mol} \% \mathrm{P}^{n} \mathrm{Bu}_{3}$ LiO ${ }^{t} \mathrm{Bu}(2.2$ equiv)

$\operatorname{THF}(0.25 \mathrm{M})$ $60^{\circ} \mathrm{C}, 16 \mathrm{~h}$

A 20-mL glass vessel equipped with J. Young ${ }^{\circledR}$ O-ring tap containing a magnetic stirring bar, TsNHNH2 (149.0 mg, $0.80 \mathrm{mmol}, 2.0$ equiv), carbonyl compound (107.3 mg, $0.80 \mathrm{mmol}, 2.0$ equiv), methanol $(3.2 \mathrm{~mL})$ was heated to $60{ }^{\circ} \mathrm{C}$. After approximately 5-30 minutes, 2a was obtained as crude product. The precipitate was dried in vacuo. To this vessel were added $\mathrm{Pd}(\mathrm{OAc})_{2}(9.0 \mathrm{mg}, 0.040 \mathrm{mmol}$, $10 \mathrm{~mol} \%$ ), LiO ${ }^{t} \mathrm{Bu}(70.5 \mathrm{mg}, 0.88 \mathrm{mmol}, 2.2$ equiv) and thioester $\mathbf{1 A}(85.7 \mathrm{mg}, 0.40 \mathrm{mmol}, 1.0$ equiv). The vessel was vacuumed and refilled $\mathrm{N}_{2}$ gas three times, and then added $\mathrm{P}^{n} \mathrm{Bu}_{3}(20.0 \mu \mathrm{L}, 0.040 \mathrm{mmol}$, $20 \mathrm{~mol} \%$ ) and THF $(1.6 \mathrm{~mL})$. The vessel was sealed with O-ring tap and then heated at $60{ }^{\circ} \mathrm{C}$ for $16 \mathrm{~h}$ with stirring. After cooling the reaction mixture to room temperature, the mixture was passed through a short silica-gel pad with EtOAc as an eluent, and then concentrated in vacuo. The residue was purified by Isolera $^{\circledR}$ (hexane) to afford the corresponding alkenyl sulfide 3Aa $(71.9 \mathrm{mg}, 79 \%, Z / E=$ 93:7).

\section{Detection of Benzaldehyde using $1 \mathrm{~K}$}




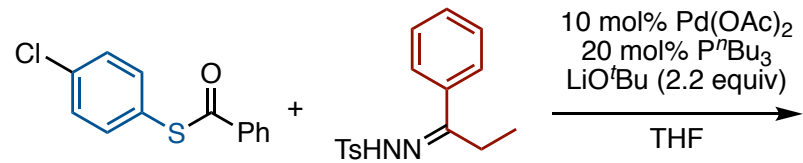

$1 K$

2a (2.0 equiv)<smiles>C/C=C(/Sc1ccc(Cl)cc1)c1ccccc1</smiles>

3Ка $91 \%$<smiles>O=Cc1ccccc1</smiles>

8

According to a crude NMR after the reaction of $1 \mathbf{K}$ with $\mathbf{2 a}$, the generation of $\mathbf{3 K a}$ along with benzaldehyde 8 as a co-product was observed. ${ }^{1} \mathrm{H}$ NMR peaks of $\mathbf{8}$ were indicated as green asterisks in following Figure S1 (red: 3Ka).

${ }^{1} \mathrm{H}$ NMR of benzaldehyde 8: $\delta 10.0(\mathrm{~s}, 1 \mathrm{H}), 7.89(\mathrm{~d}, J=8.0 \mathrm{~Hz}, 2 \mathrm{H}), 7.64(\mathrm{t}, J=8.0 \mathrm{~Hz}, 1 \mathrm{H}), 7.54(\mathrm{t}, J$ $=8.0 \mathrm{~Hz}, 2 \mathrm{H})$.

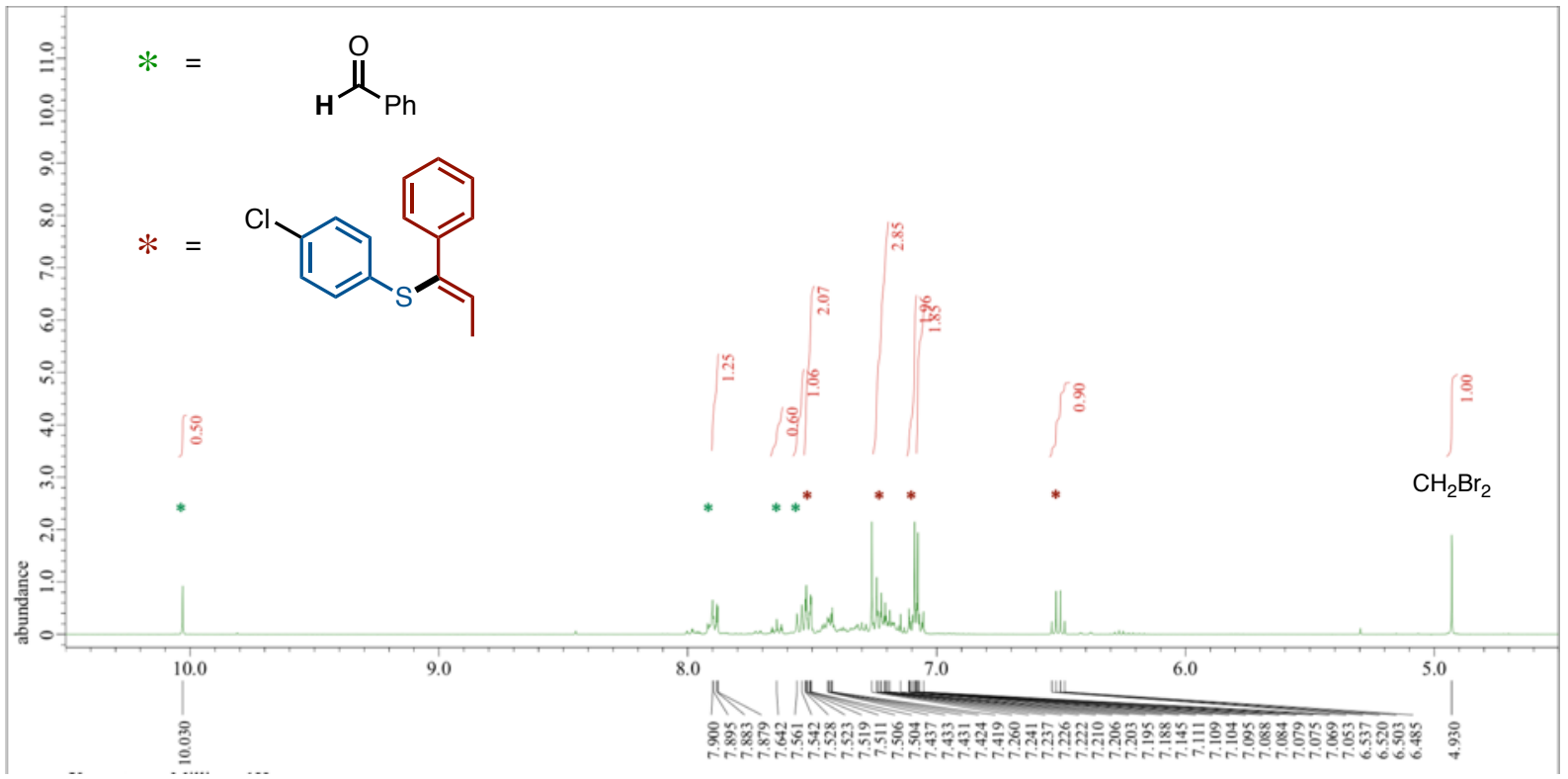

Figure S1. Crude ${ }^{1} \mathrm{H}$ NMR after the catalytic reaction of $\mathbf{1 K}$ with $\mathbf{2 a}$. 


\section{Derivatization of Product}<smiles>CC(=Cc1ccccc1)Sc1ccc(Br)cc1</smiles>

3Ja<smiles>COc1ccc([Hg]O)cc1</smiles>

1.5 equiv

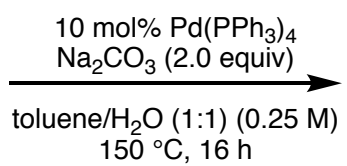
$150^{\circ} \mathrm{C}, 16 \mathrm{~h}$

A 20-mL glass vessel equipped with J. Young ${ }^{\circledR}$ O-ring tap containing a magnetic stirring bar and $\mathrm{Na}_{2} \mathrm{CO}_{3}\left(21.2 \mathrm{mg}, 0.20\right.$ mmol, 2.0 equiv) was dried with a heat-gun in vacuo and filled with $\mathrm{N}_{2}$ after cooling to room temperature. To this vessel were added $\mathrm{Pd}\left(\mathrm{PPh}_{3}\right)_{4}(11.6 \mathrm{mg}, 0.010 \mathrm{mmol}, 10 \mathrm{~mol} \%)$, 4-methoxyphenyl boronic acid (22.8 mg, $0.15 \mathrm{mmol}, 1.5$ equiv) and 3Ja (37.1 $\mathrm{mg} 0.12 \mathrm{mmol}, 1.0$ equiv). The vessel was vacuumed and refilled $\mathrm{N}_{2}$ gas three times, and then added toluene/ $\mathrm{H}_{2} \mathrm{O}(1: 1)$ $(0.4 \mathrm{~mL})$. The vessel was sealed with O-ring tap and then heated at $150{ }^{\circ} \mathrm{C}$ for $16 \mathrm{~h}$ with stirring. After cooling the reaction mixture to room temperature, the mixture was passed through a short silica-gel pad with EtOAc as an eluent, and then concentrated in vacuo. The residue was purified by PTLC (hexane $/ \mathrm{EtOAc}=97: 3)$ to afford $\mathbf{5}(38.0 \mathrm{mg}, 94 \%, \boldsymbol{Z} / \boldsymbol{E}=85: 15)$ as a colorless oil.

For $Z$ isomer: 5: ${ }^{1} \mathrm{H}$ NMR (400 MHz, $\left.\mathrm{CDCl}_{3}\right) \delta 7.57(\mathrm{~d}, J=7.2 \mathrm{~Hz}, 2 \mathrm{H}), 7.41(\mathrm{~d}, J=8.8 \mathrm{~Hz}, 2 \mathrm{H}), 7.32$ $(\mathrm{d}, J=8.0 \mathrm{~Hz}, 2 \mathrm{H}), 7.29-7.22(\mathrm{~m}, 2 \mathrm{H}), 7.21-7.15(\mathrm{~m}, 3 \mathrm{H}), 6.91(\mathrm{~d}, J=8.8 \mathrm{~Hz}, 2 \mathrm{H}), 6.54(\mathrm{q}, J=6.8$ $\mathrm{Hz}, 1 \mathrm{H}), 3.80(\mathrm{~s}, 3 \mathrm{H}), 2.10(\mathrm{~d}, J=6.8 \mathrm{~Hz}, 3 \mathrm{H}) ;{ }^{13} \mathrm{C} \mathrm{NMR}\left(101 \mathrm{MHz}, \mathrm{CDCl}_{3}\right) \delta 159.0,140.6,137.8$, 134.8, 134.3, 134.0, 132.9, 128.2, 128.1, 127.7, 127.4, 127.3, 126.9, 114.1, 55.3, 16.9; HRMS (DART) $m / z$ calcd for $\mathrm{C}_{22} \mathrm{H}_{21} \mathrm{OS}[\mathrm{M}+\mathrm{H}]^{+}: 333.1308$ found 333.1303.

For $E$ isomer: ${ }^{1} \mathrm{H}$ NMR $\left(400 \mathrm{MHz}, \mathrm{CDCl}_{3}\right) \delta 7.46-7.40(\mathrm{~m}, 2 \mathrm{H}), 7.41(\mathrm{~d}, J=8.8 \mathrm{~Hz}, 2 \mathrm{H}), 7.38-7.35$ $(\mathrm{m}, 2 \mathrm{H}), 7.32(\mathrm{~d}, J=8.0 \mathrm{~Hz}, 2 \mathrm{H}), 7.29-7.22(\mathrm{~m}, 2 \mathrm{H}), 7.21-7.15(\mathrm{~m}, 1 \mathrm{H}), 6.93-6.88(\mathrm{~m}, 2 \mathrm{H}), 6.24(\mathrm{q}$, $J=7.6 \mathrm{~Hz}, 1 \mathrm{H}), 3.81(\mathrm{~s}, 3 \mathrm{H}), 1.78(\mathrm{~d}, J=7.2 \mathrm{~Hz}, 3 \mathrm{H})$. 


\section{Effect of Parameters}

\section{8-1. Screening of Solvents}

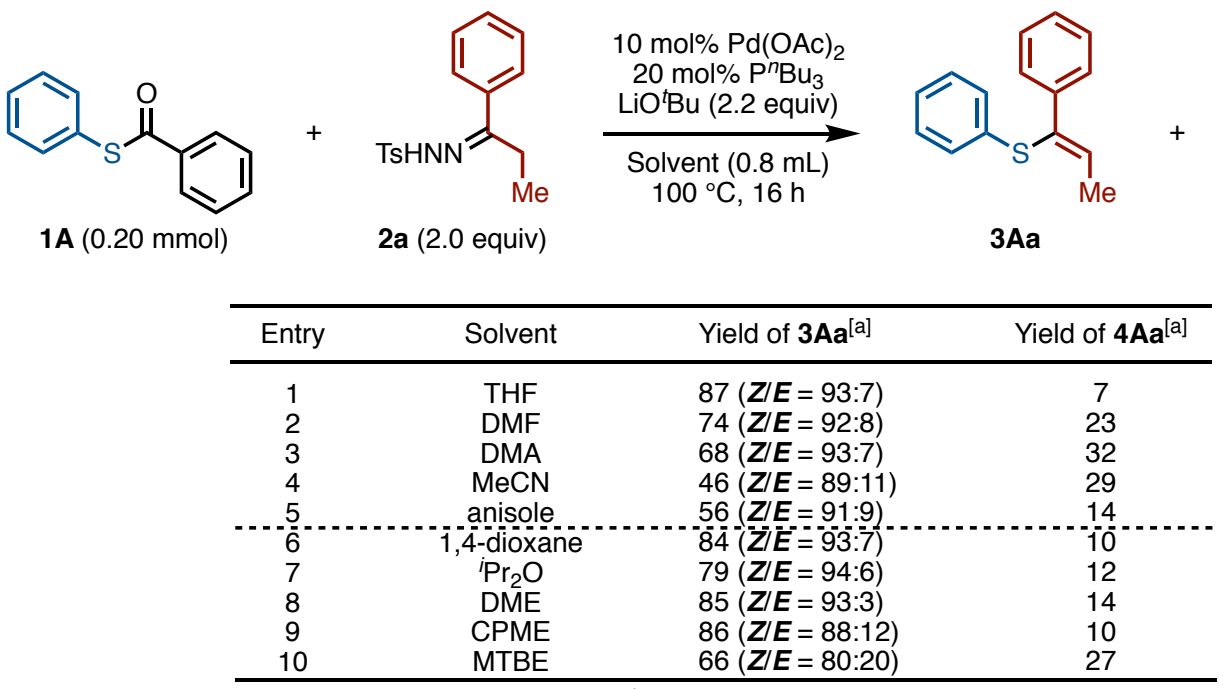

[a] Yields were determined by ${ }^{1} \mathrm{H}$ NMR using $\mathrm{CH}_{2} \mathrm{Br}_{2}$ as an internal standard.

\section{8-2. Screening of Ligands}<smiles>O=C(Sc1ccccc1)c1ccccc1</smiles>

1 A (0.20 mmol)<smiles>CCC(=N)c1ccccc1</smiles>

2a (2.0 equiv)

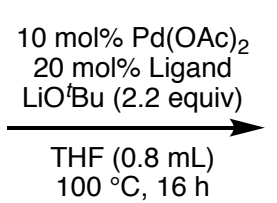

$100^{\circ} \mathrm{C}, 16 \mathrm{~h}$

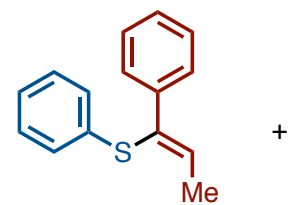

3Aa<smiles>CCC(Sc1ccccc1)c1ccccc1</smiles>

$4 \mathrm{Aa}$

\begin{tabular}{|c|c|c|c|}
\hline Entry & Ligand & Yield of $\mathbf{3} \mathbf{A a}^{[\mathrm{a}]}$ & Yield of $\mathbf{4} \mathbf{A a}^{[a]}$ \\
\hline 1 & BrettPhos & $23(Z / E=83: 17)$ & 20 \\
\hline 2 & XPhos & $33(Z / E=82: 18)$ & 20 \\
\hline 3 & bipy & $18(Z / E=83: 17)$ & 13 \\
\hline 4 & dcypt & $81(Z / E=90: 10)$ & 7 \\
\hline 5 & dcype & $72(Z / E=90: 0)$ & 13 \\
\hline & $\overline{\mathrm{P}} \overline{\mathrm{Cy}} \mathrm{y}_{2} \mathrm{P} \overline{\mathrm{P}}$ & $36(Z / E=92: 8)$ & 12 \\
\hline 7 & $\mathrm{PMePh}_{2}$ & $40(Z / E=90: 10)$ & 13 \\
\hline 8 & $\mathrm{PPh}_{3}$ & $23(Z / E=91: 9)$ & 14 \\
\hline 9 & dppp & $34(Z / E=91: 19$ & 16 \\
\hline 10 & $\mathrm{P}^{n} \mathrm{Bu}_{3}$ & $87(Z / E=93: 7)$ & 7 \\
\hline $11^{-}$ & $\left.\mathrm{P}^{n} \mathrm{Hexy}\right|_{3}$ & $85(Z / E=92: 8)$ & $-\overline{9}$ \\
\hline 12 & $\mathrm{P}^{n} \mathrm{Octyl}_{3}$ & $87(\boldsymbol{Z} / \bar{E}=92: 8)$ & 3 \\
\hline 13 & $\mathrm{PAd}_{3}$ & $18(Z / E=83: 17)$ & 12 \\
\hline 14 & $\mathrm{P}^{t} \mathrm{Bu}_{3} \mathrm{HBF}_{4}$ & $9(Z / E=67: 33)$ & 3 \\
\hline 15 & $\mathrm{PMe}_{3} \mathrm{HBF}_{4}$ & $26(Z / E=81: 19)$ & 7 \\
\hline 16 & $\mathrm{PC} \mathrm{y}_{3} \mathrm{HBF}$ & $25(Z I \bar{E}=84: 16)$ & 6 \\
\hline 17 & Amphos & $9(Z / E=67: 33)$ & 3 \\
\hline 18 & $\mathrm{Pd}(\mathrm{IMes}) \mathrm{Cl}$ & $30(Z / E=97: 3)$ & 18 \\
\hline 19 & none & $29(Z / E=83: 17)$ & 25 \\
\hline
\end{tabular}

[a] Yields were determined by ${ }^{1} \mathrm{H}$ NMR using $\mathrm{CH}_{2} \mathrm{Br}_{2}$ as an internal standard. 


\section{8-3. Screening of Temperature}

\begin{tabular}{|c|c|c|c|c|}
\hline $1 \mathrm{~A}(0.20 \mathrm{mmol})$ & & Me & 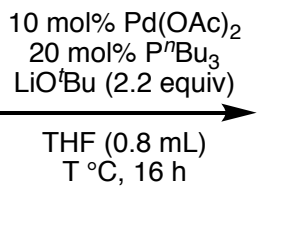 & Me \\
\hline & Entry & $\mathrm{T}$ & Yield of $\mathbf{3} \mathbf{A a}^{[\mathrm{a}]}$ & Yield of $4 \mathbf{A a}^{[a]}$ \\
\hline & $\begin{array}{l}1 \\
2 \\
3 \\
4 \\
5\end{array}$ & $\begin{array}{l}150 \\
100 \\
80 \\
60 \\
40\end{array}$ & $\begin{array}{c}90(Z / E=95: 5) \\
87(Z / E=93: 7) \\
95(Z / E=94: 6) \\
93(Z / E=95: 5) \\
20(Z / E=85: 15)\end{array}$ & $\begin{array}{l}8 \\
7 \\
5 \\
7 \\
4\end{array}$ \\
\hline
\end{tabular}

[a] Yields were determined by ${ }^{1} \mathrm{H}$ NMR using $\mathrm{CH}_{2} \mathrm{Br}_{2}$ as an internal standard.

\section{8-4. Screening of Bases}<smiles>O=C(Sc1ccccc1)c1ccccc1</smiles>

$1 \mathrm{~A}(0.20 \mathrm{mmol})$<smiles>CCC(=N)c1ccccc1</smiles>

2a (2.0 equiv)

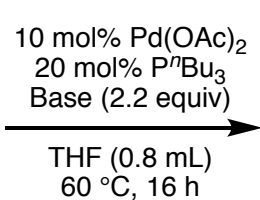

$60^{\circ} \mathrm{C}, 16 \mathrm{~h}$<smiles>CC=C(Sc1ccccc1)c1ccccc1</smiles>

3Aa<smiles>[M]CC(Sc1ccccc1)c1ccccc1</smiles>

$4 \mathrm{Aa}$

\begin{tabular}{|c|c|c|c|}
\hline Entry & Base & Yield of $\mathbf{3 A a ^ { [ a ] }}$ & Yield of $\mathbf{4} \mathbf{A a}^{[\mathrm{a}]}$ \\
\hline 1 & $\mathrm{LiO}^{t} \mathrm{Bu}$ & $93(Z / E=95: 5)$ & 7 \\
\hline 2 & $\mathrm{NaO}{ }^{t} \mathrm{Bu}$ & $69(Z / E=93: 7)$ & 31 \\
\hline 3 & $\mathrm{KO}^{t} \mathrm{Bu}$ & $61(Z / E=92: 8)$ & 37 \\
\hline 4 & $\mathrm{Mg}\left(\mathrm{O}^{t} \mathrm{Bu}\right)_{2}$ & $11(Z / E=100: 0)$ & 0 \\
\hline$-\frac{5}{6}$ & $\begin{array}{l}\text { none } \\
\text { Koñ }\end{array}$ & - & $\begin{array}{l}0 \\
5\end{array}$ \\
\hline 7 & $\mathrm{Na}_{2} \mathrm{CO}_{3}$ & $6(Z / E=67: 33)$ & 0 \\
\hline 8 & $\mathrm{Li}_{2} \mathrm{CO}_{3}$ & $2(Z / E=100: 0)$ & 0 \\
\hline 9 & $\mathrm{Cs}_{2} \mathrm{CO}_{3}$ & $73(Z / E=95: 5$ & 18 \\
\hline 10 & $\mathrm{Li}_{3} \mathrm{PO}_{4}$ & 0 & 0 \\
\hline 11 & $\mathrm{Na}_{3} \mathrm{PO}_{4}$ & $2 \overline{2}(Z \bar{E}=\overline{9} \overline{5}: \overline{5})-$ & $\overline{0}^{-}$ \\
\hline 12 & $\mathrm{~K}_{3} \mathrm{PO}_{4}$ & $85(Z / E=95: 5)$ & 5 \\
\hline 13 & $\mathrm{KHCO}_{3}$ & $17(Z / E=100: 0)$ & 0 \\
\hline 14 & $\mathrm{NaHCO}_{3}$ & $1(Z / E=100: 0)$ & 0 \\
\hline 15 & $\mathrm{NaH}$ & $45(Z / E=91: 9)$ & 6 \\
\hline 16 & $\mathrm{KOH}$ & $83(Z / E=85: 15$ & 17 \\
\hline 17 & $\mathrm{NaOH}$ & $77(Z / E=95: 5)$ & 21 \\
\hline 18 & $\mathrm{Et}_{3} \mathrm{~N}$ & 0 & 0 \\
\hline 19 & DIPEA & 0 & 0 \\
\hline 20 & $\mathrm{NaOAc}$ & 0 & 0 \\
\hline 21 & KOAC & 0 & 0 \\
\hline
\end{tabular}

[a] Yields were determined by ${ }^{1} \mathrm{H}$ NMR using $\mathrm{CH}_{2} \mathrm{Br}_{2}$ as an internal standard. 


\section{Reference}

[1] Ichiishi, N.; Malapit, C. A.; Wozniak, Ł.; Sanford, M. S. Palladium- and Nickel-Catalyzed Decarbonylative C-S Coupling to Convert Thioesters to Thioethers. Org. Lett. 2018, 20, 44-47.

[2] Singh, P.; Peddinti, R. K. Harnessing the Catalytic Behaviour of 1,1,1,3,3,3-Hexafluoro-2-propanol (HFIP): An Expeditious Synthesis of Thioesters. Tetrahedron Lett. 2017, 19, 1875-1578.

[3] Liu, C.; Szostak, M. Decarbonylative Thioetherification by Nickel Catalysis using Air- and Moisture-Stable Nickel Precatalysts. Chem. Commun. 2018, 54, 2130-2133.

[4] Qiao, Z.-J.; Jiang, X.-F. Ligand-Controlled Divergent Cross-Coupling Involving Organosilicon Compounds for Thioether and Thioester Synthesis. Org. Lett. 2016, 18, 1550-1553.

[5] Chen, C.-T.; Kuo, J.-H.; Li, C.-H.; Barhate, N. B.; Hon, S.-W.; Li, T.-W.; Chao, S.-D.; Liu, C.-C.; Li, Y.-C.; Chang, I.-H.; Lin, J.-S.; Liu, C.-J.; Chou, Y.-C. Catalytic Nucleophilic Acyl Substitution of Anhydrides by Amphoteric Vanadyl Triflate. Org. Lett. 2001, 3, 3729-3732.

[6] Yu, Y.; Liebeskind, L. S. Copper-Mediated, Palladium-Catalyzed Coupling of Thiol Esters with Aliphatic Organoboron Reagents. J. Org. Chem.2004, 69, 3554-3557.

[7] Cao, H.; McNamee, L.; Alper, H. Palladium-Catalyzed Thiocarbonylation of Iodoarenes with Thiols in Phosphonium Salt Ionic Liquids. J. Org. Chem. 2008, 73, 3530-3534.

[8] Celebi, S.; Leyva, S.; Modarelli, D. A.; Platz, M. S. 1,2-Hydrogen Migration and Alkene Formation in the Photoexcited States of Alkylphenyldiazomethanes. J. Am. Chem. Soc. 1993, 115, 8613-8620.

[9] Hatcher, J. M.; Coltart, D. M. Copper(I)-Catalyzed Addition of Grignard Reagents to in Situ-Derived N-Sulfonyl Azoalkenes: An Umpolung Alkylation Procedure Applicable to the Formation of Up to Three Contiguous Quaternary Centers. J. Am. Chem. Soc. 2010, 132, 4546-4547.

[10] Ye, F.; Ma, X.; Xiao, Q.; Li, H.; Zhang, Y.; Wang, J. C(sp)-C(sp $\left.{ }^{3}\right)$ Bond Formation through $\mathrm{Cu}$-Catalyzed Cross-Coupling of N-Tosylhydrazones and Trialkylsilylethynes. J. Am. Chem. Soc. 2012, 134, 5742-5745.

[11] Chen, J.; Chen, S.; Xu, X.; Tang, Z.; Au, C.-T.; Qiu, R. Nickel-Catalyzed Regioselective Cleavage of $\mathrm{C}_{\mathrm{sp} 2}-\mathrm{S}$ Bonds: Method for the Synthesis of Tri- and Tetrasubstituted Alkenes. J. Org. Chem. 2016, 81, 3246-3255.

[12] [a] Taniguchi, T.; Fujii, T.; Idota, A.; Ishibashi, H. Reductive Addition of the Benzenethiyl Radical to Alkynes by Amine-Mediated Single Electron Transfer Reaction to Diphenyl Disulfide. Org. Lett. 2009, 11, 3298-3301. [b] Kuniyasu, H.; Yamashita, F.; Hirai, T.; Ye, J.-H.; Fujiwara, S.-i.; Kambe N. Platinum-Catalyzed Reaction of Alkynes with ArI (Ar = aryl and thienyl) and $\operatorname{Ar}{ }^{`} \mathrm{SM}(\mathrm{M}=\mathrm{Na}, \mathrm{K}$, and $\left.\mathrm{Sn}(\mathrm{Bu}-n)_{3}\right)$ : Three- vs Two-Component Cross-Coupling Reaction. Organometallics 2006, 25, 566-570. [c] Yang, Z.; Chen, X.; Kong, W.; Xia, S.; Zheng, R.; Luo. F.; Zhu, G. An Operationally Simple Approach to (E)- $\alpha$-Halo Vinyl Sulfides and Their Applications for Accessing Stereodefined Trisubstituted Alkenes. Org. Biomol. Chem. 2013, 11, 2175-2185. 
[13] Khlebnikov, A. F.; Kostikov, R. R. Product Subclass 3: 1-Halo-1-(organochalcogeno)alk-1-enes. Science of Synthesis 2006, 24, 167-222.

[14] Ager, D. J. Synthesis of Alkenes via the Peterson Reaction. J. Chem. Soc., Perkin Trans. 1 1986, 183-194.

[15] Eom, D.; Mo, J.; Lee, P. H.; Gao, Z.; Kim, S. Synthesis of Vinyl Sulfides and Vinylamines through Catalytic Intramolecular Hydroarylation in the Presence of $\mathrm{FeCl}_{3}$ and $\mathrm{AgOTf}$. Eur. J. Org. Chem. 2013, 533-540. 
10. ${ }^{1} \mathrm{H},{ }^{13} \mathrm{C}$ NMR Spectra

${ }^{1} \mathrm{H} \mathrm{NMR}$ of $1 \mathbf{B}\left(400 \mathrm{MHz}, \mathrm{CDCl}_{3}\right)$

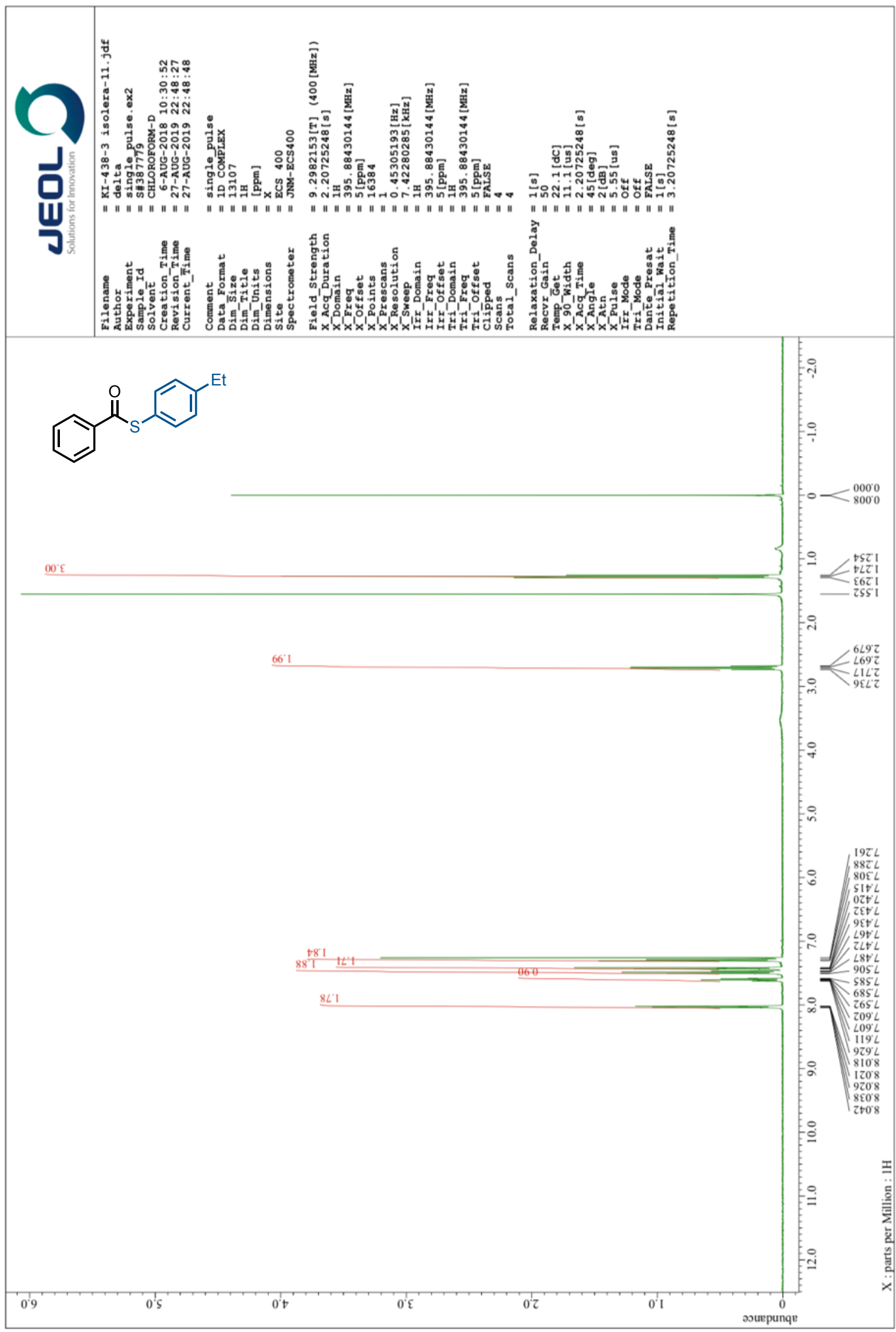


${ }^{13} \mathrm{C}$ NMR of $1 \mathbf{B}\left(101 \mathrm{MHz}, \mathrm{CDCl}_{3}\right)$

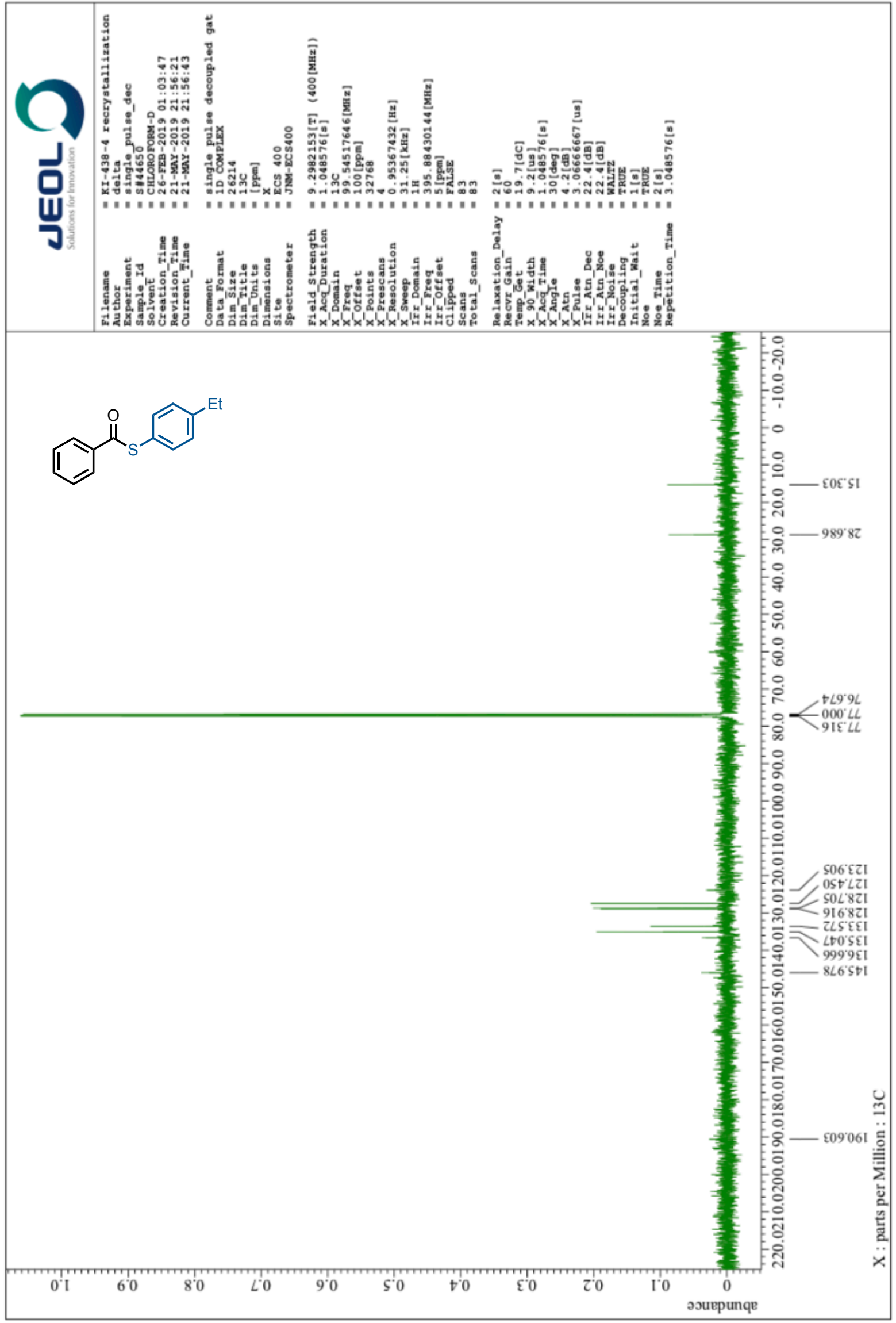


${ }^{1} \mathrm{H} \mathrm{NMR}$ of $1 \mathrm{C}\left(400 \mathrm{MHz}, \mathrm{CDCl}_{3}\right)$

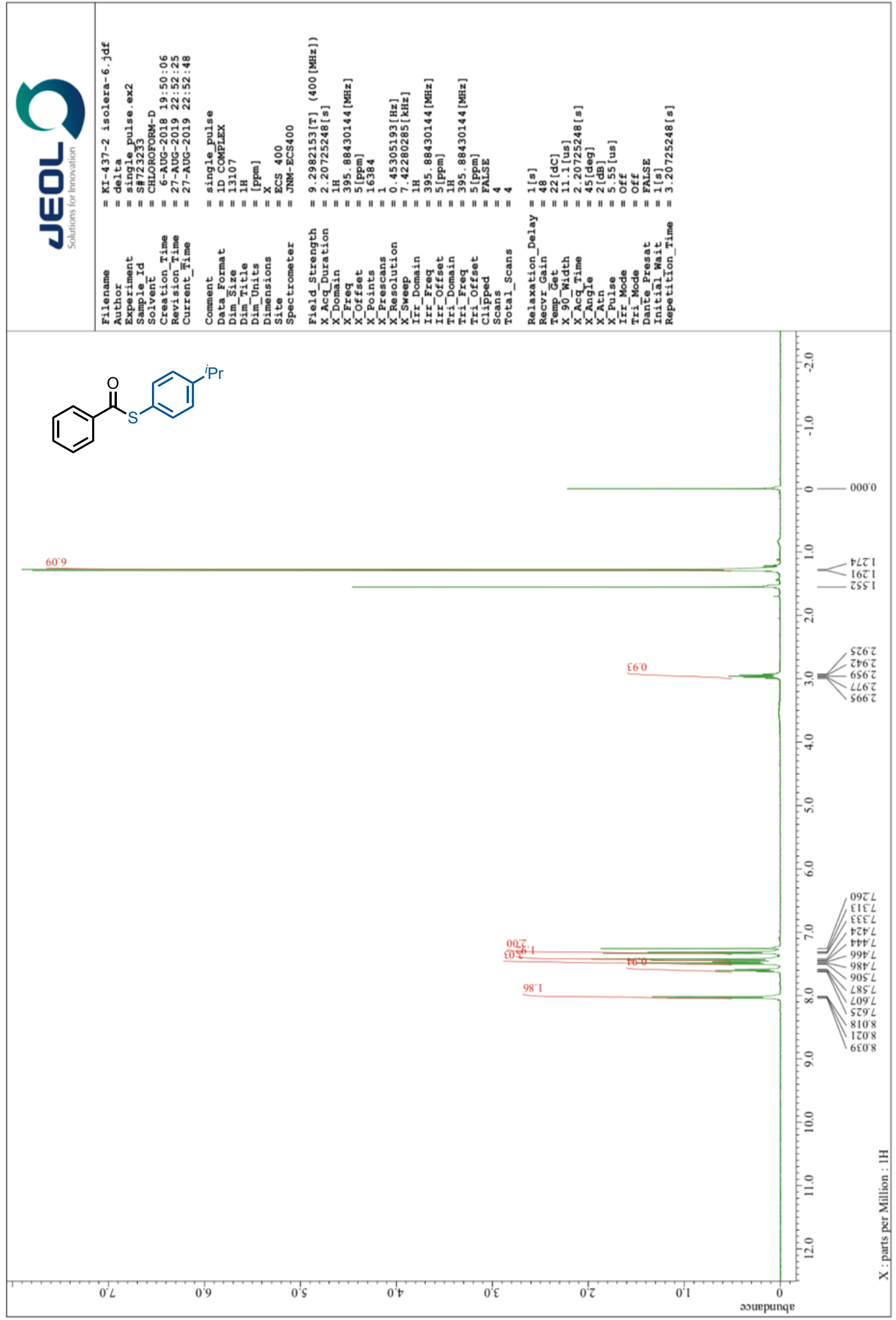


${ }^{13} \mathrm{C}$ NMR of $1 \mathrm{C}\left(101 \mathrm{MHz}, \mathrm{CDCl}_{3}\right)$

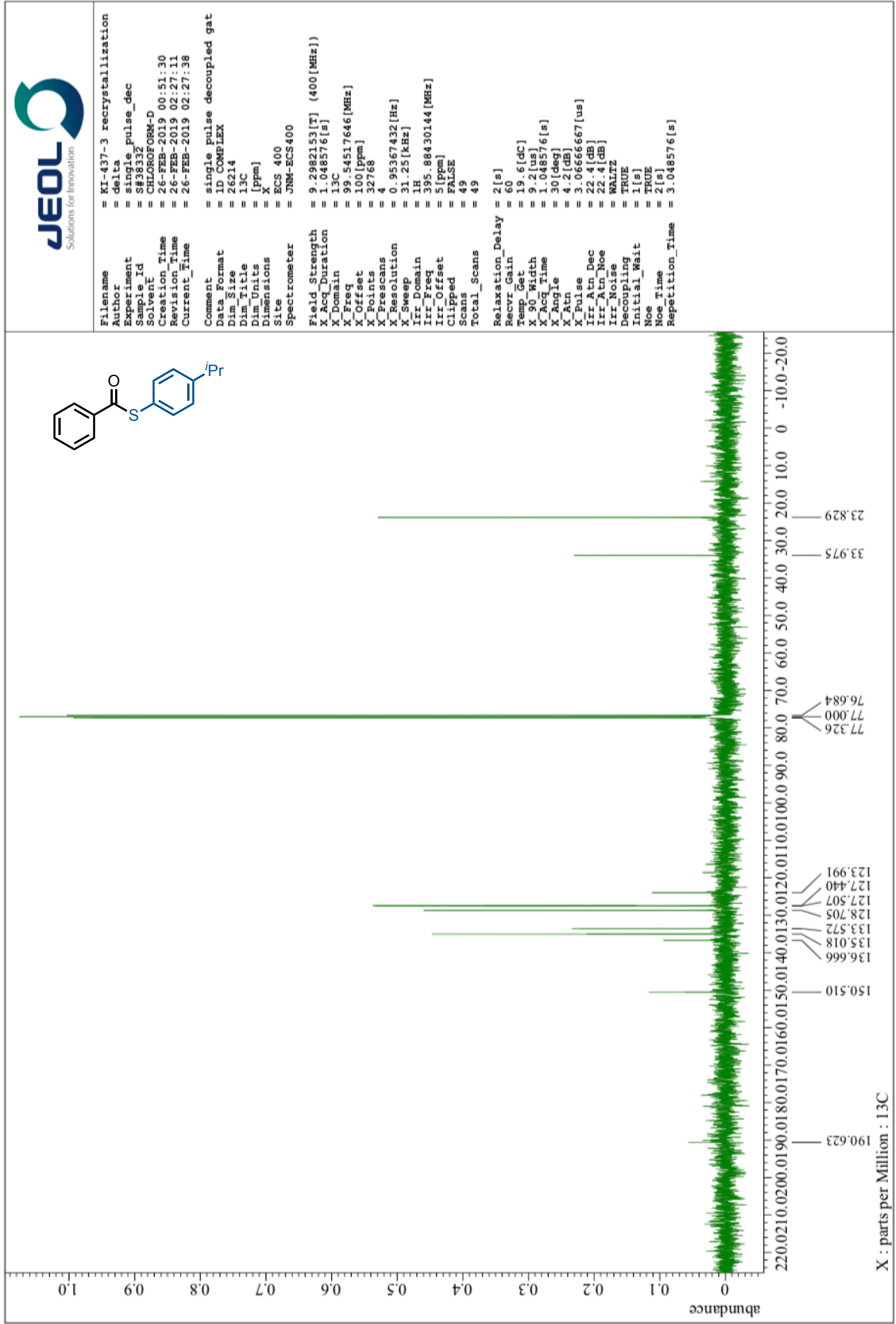


${ }^{1} \mathrm{H}$ NMR of $1 D\left(400 \mathrm{MHz}, \mathrm{CDCl}_{3}\right)$

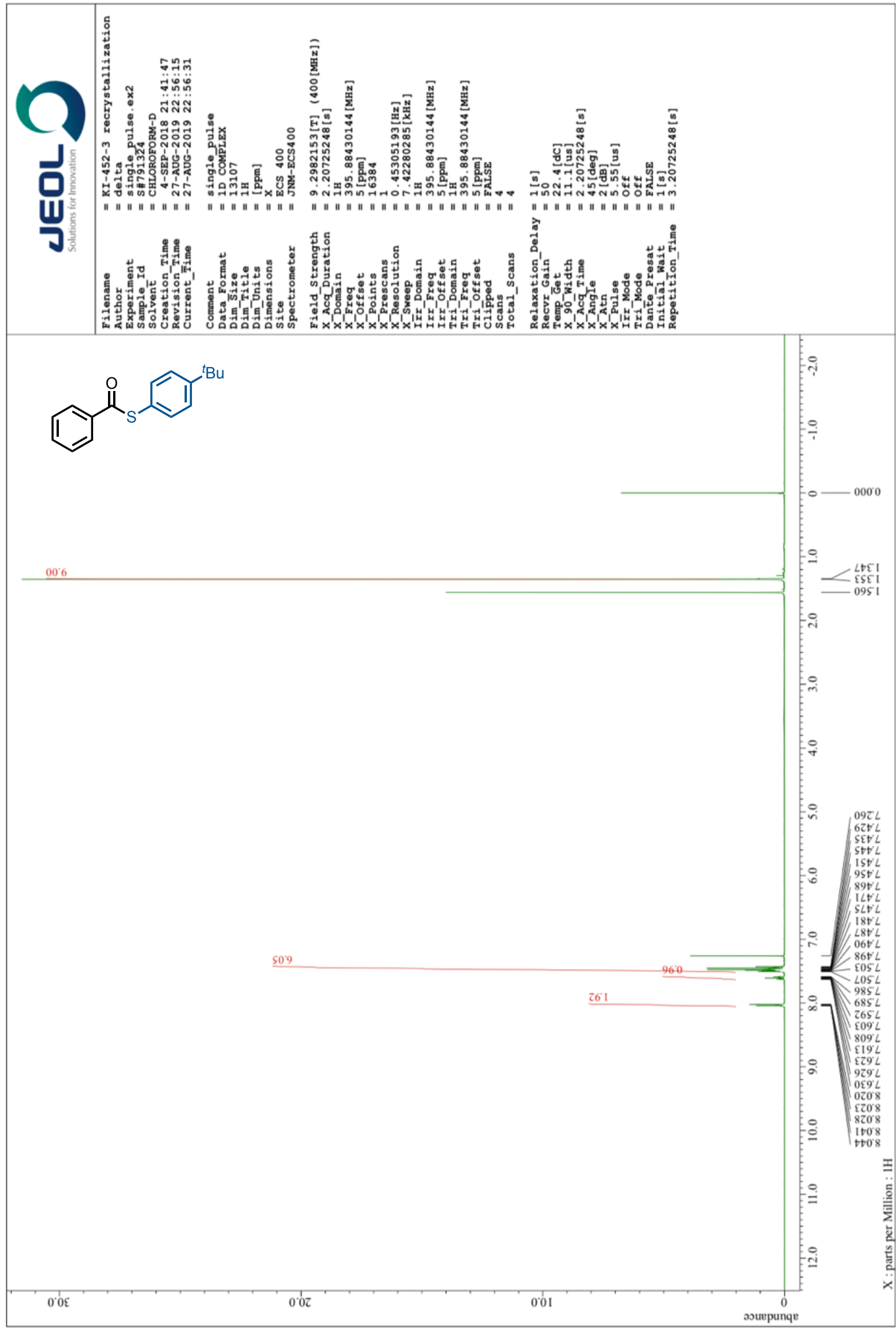


${ }^{13} \mathrm{C}$ NMR of $1 \mathrm{D}\left(101 \mathrm{MHz}, \mathrm{CDCl}_{3}\right)$

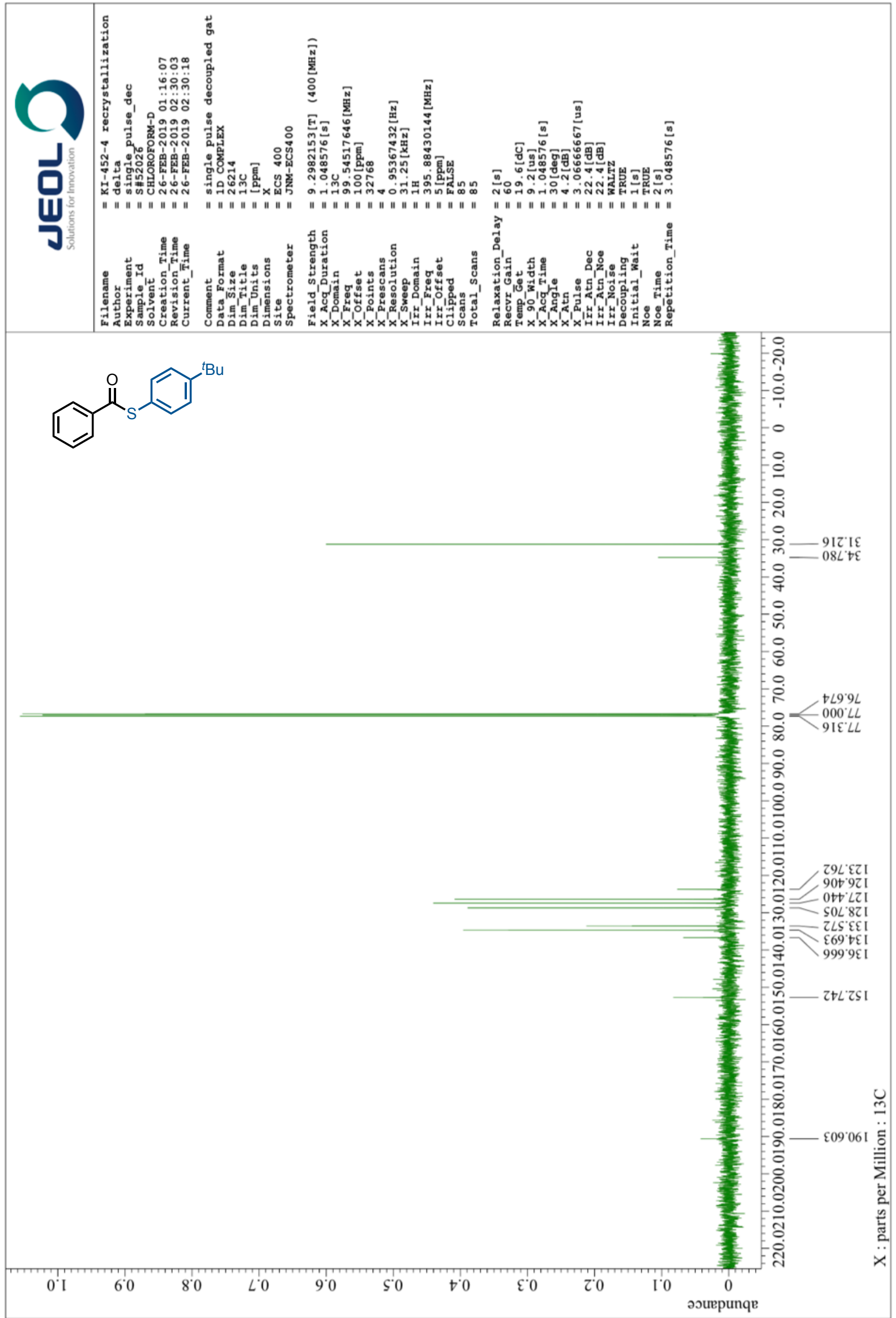


${ }^{1} \mathrm{H}$ NMR of $\mathbf{1 N}\left(400 \mathrm{MHz}\right.$, DMSO-d $\left.{ }_{6}\right)$

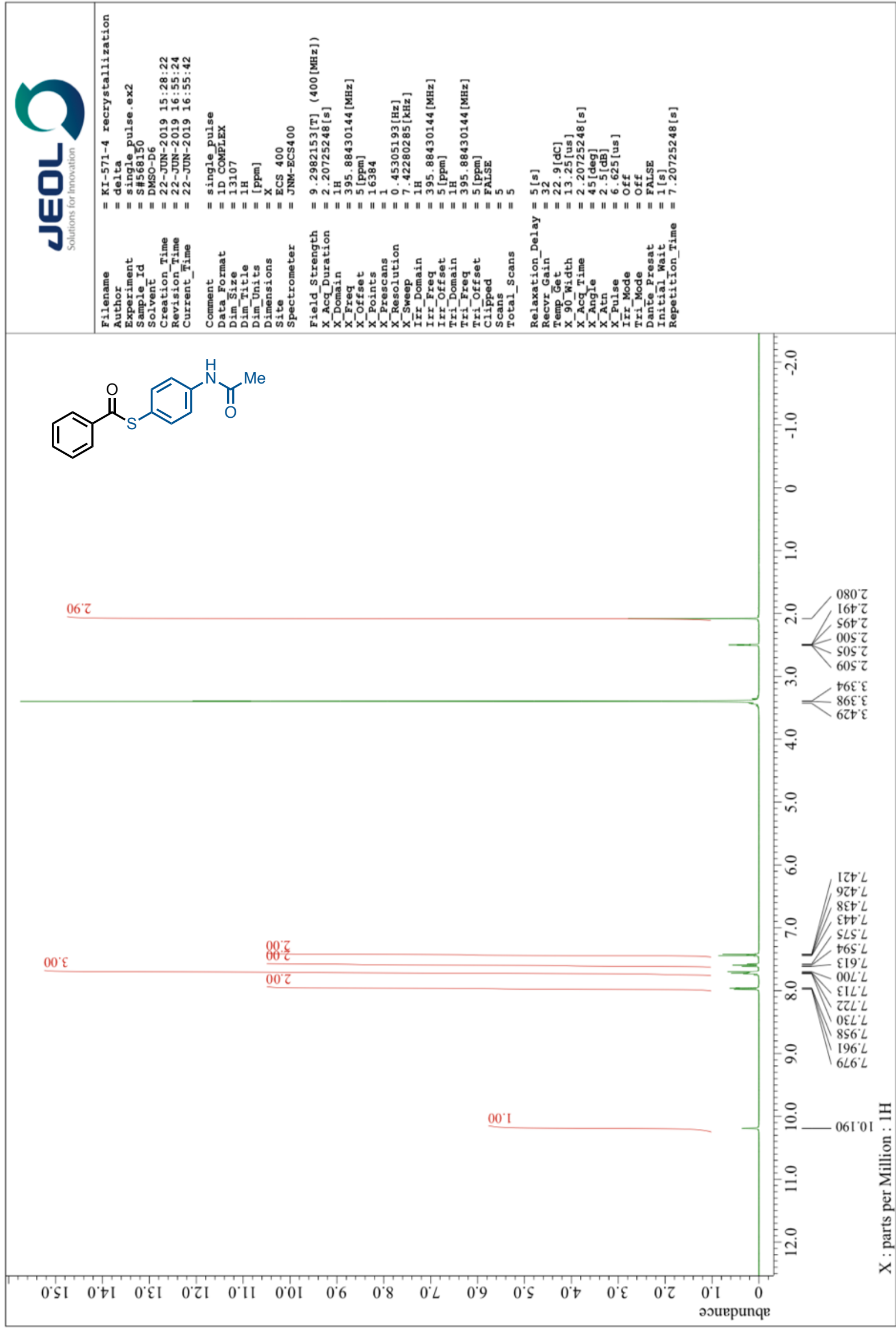


${ }^{13} \mathrm{C}$ NMR of $\mathbf{1 N}(101 \mathrm{MHz}$, DMSO-d 6 )

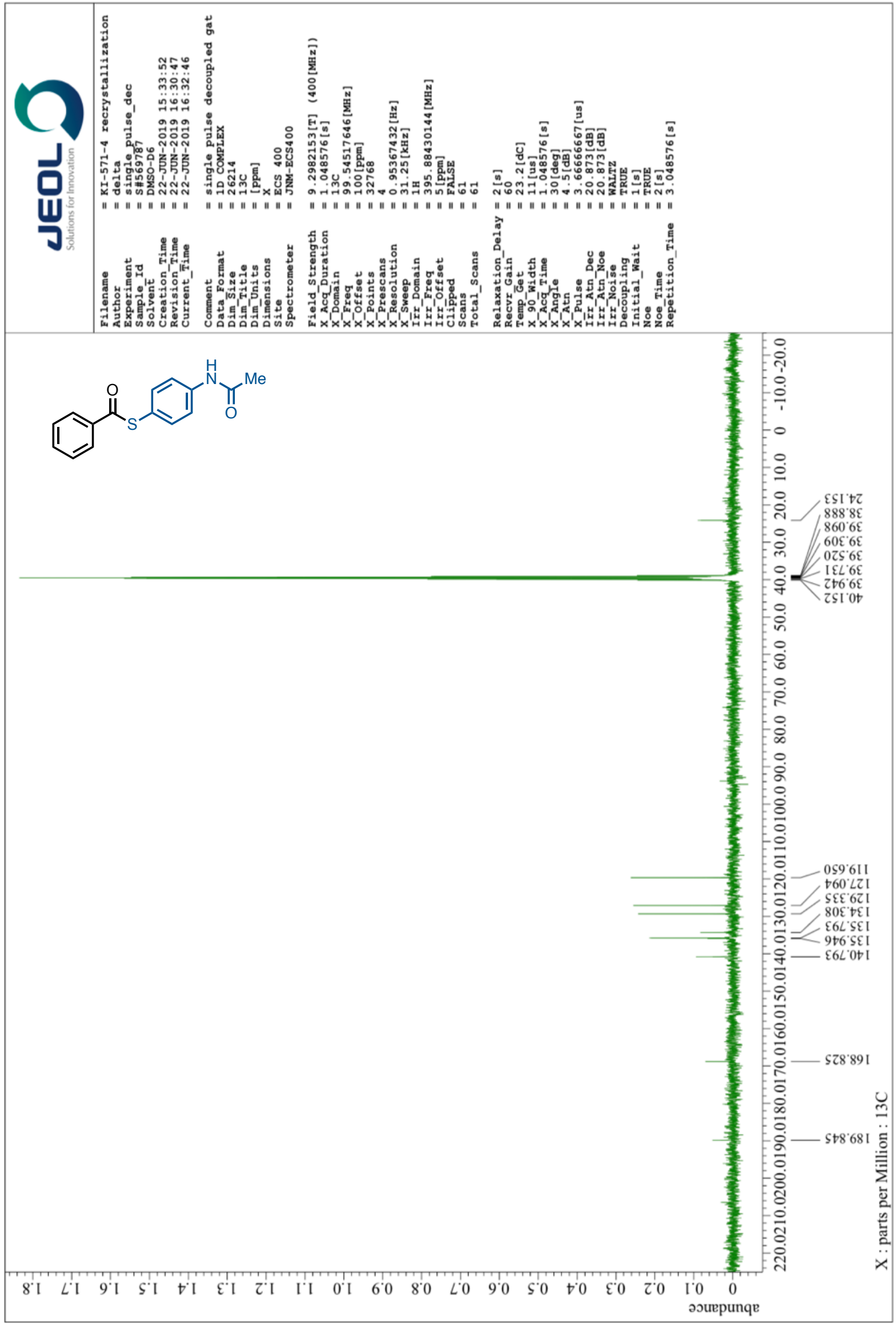


${ }^{1} \mathrm{H} \mathrm{NMR}$ of $10\left(400 \mathrm{MHz}, \mathrm{CDCl}_{3}\right)$

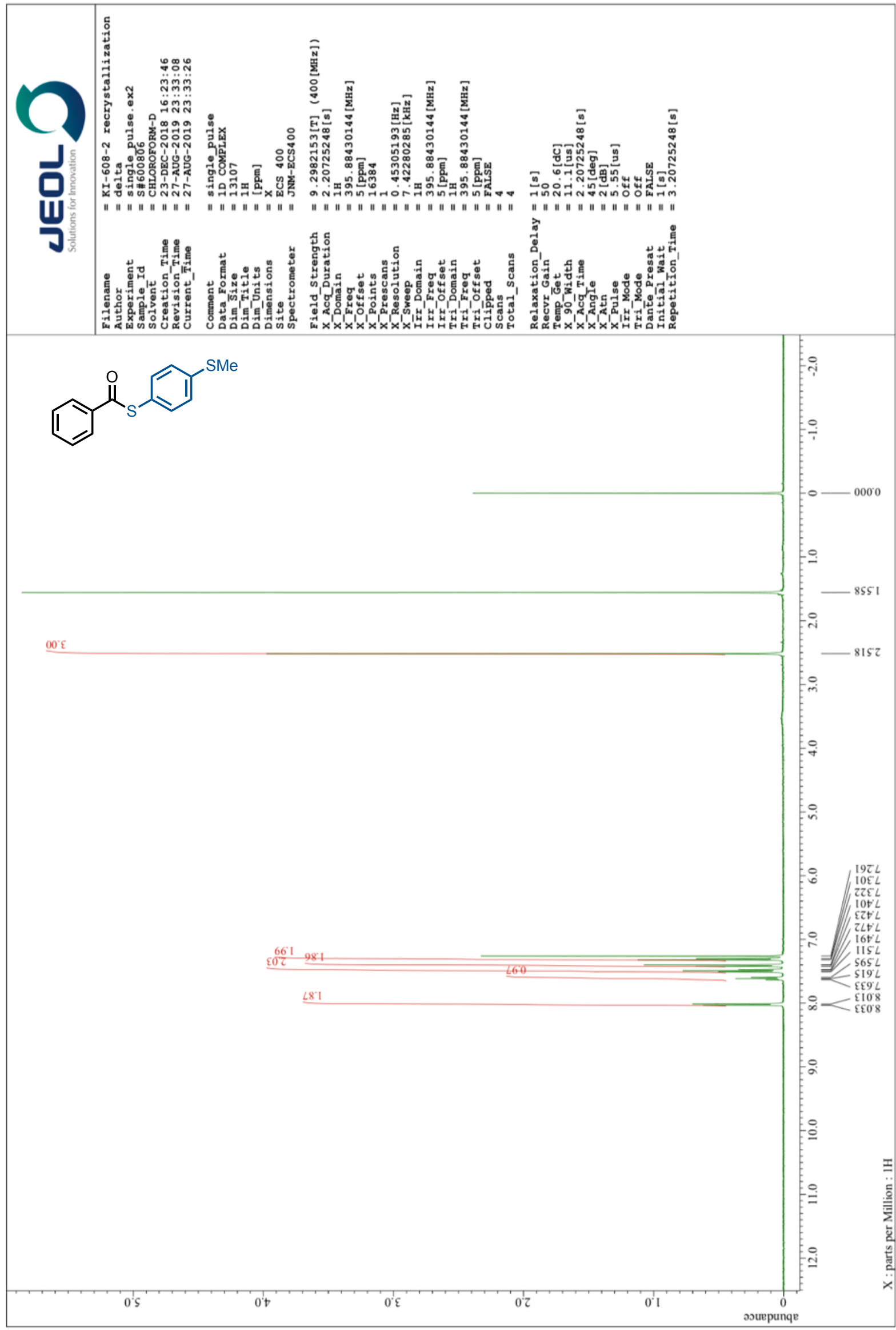


${ }^{13} \mathrm{C}$ NMR of $10\left(101 \mathrm{MHz}, \mathrm{CDCl}_{3}\right)$

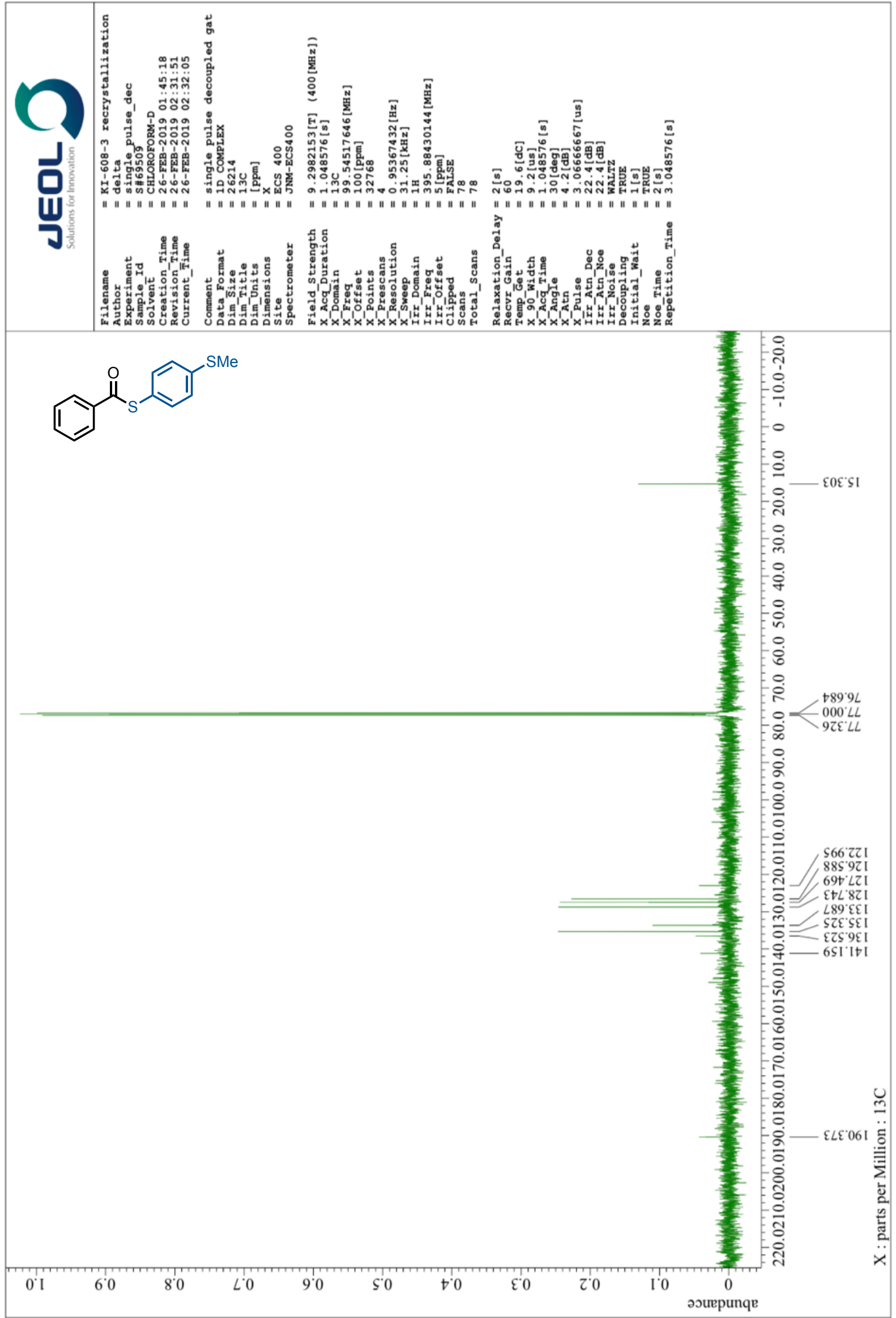


${ }^{1} \mathrm{H}$ NMR of $\mathbf{2 b}\left(400 \mathrm{MHz}, \mathrm{CDCl}_{3}\right)$

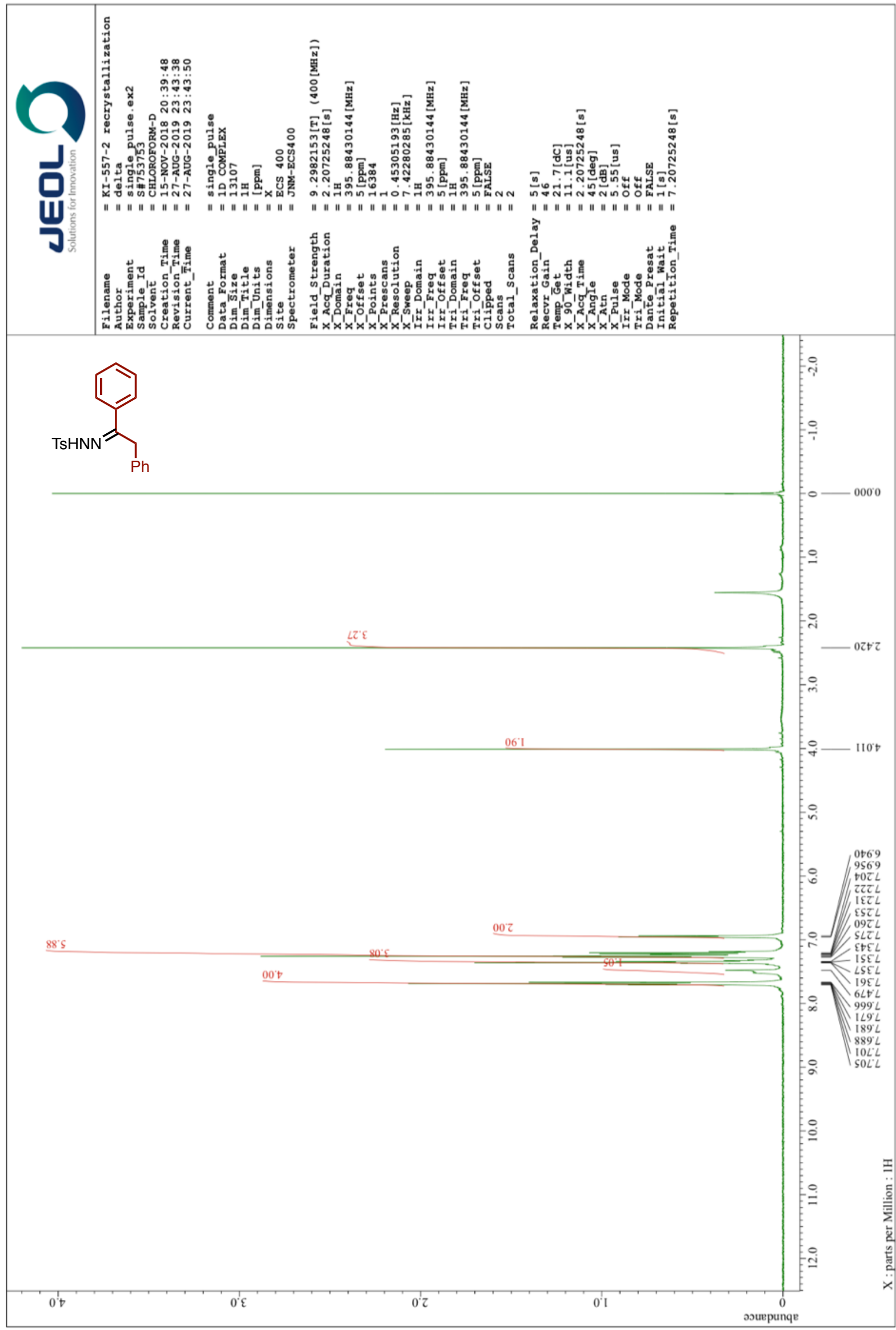


${ }^{13} \mathrm{C}$ NMR of $\mathbf{2 b}\left(101 \mathrm{MHz}, \mathrm{CDCl}_{3}\right)$

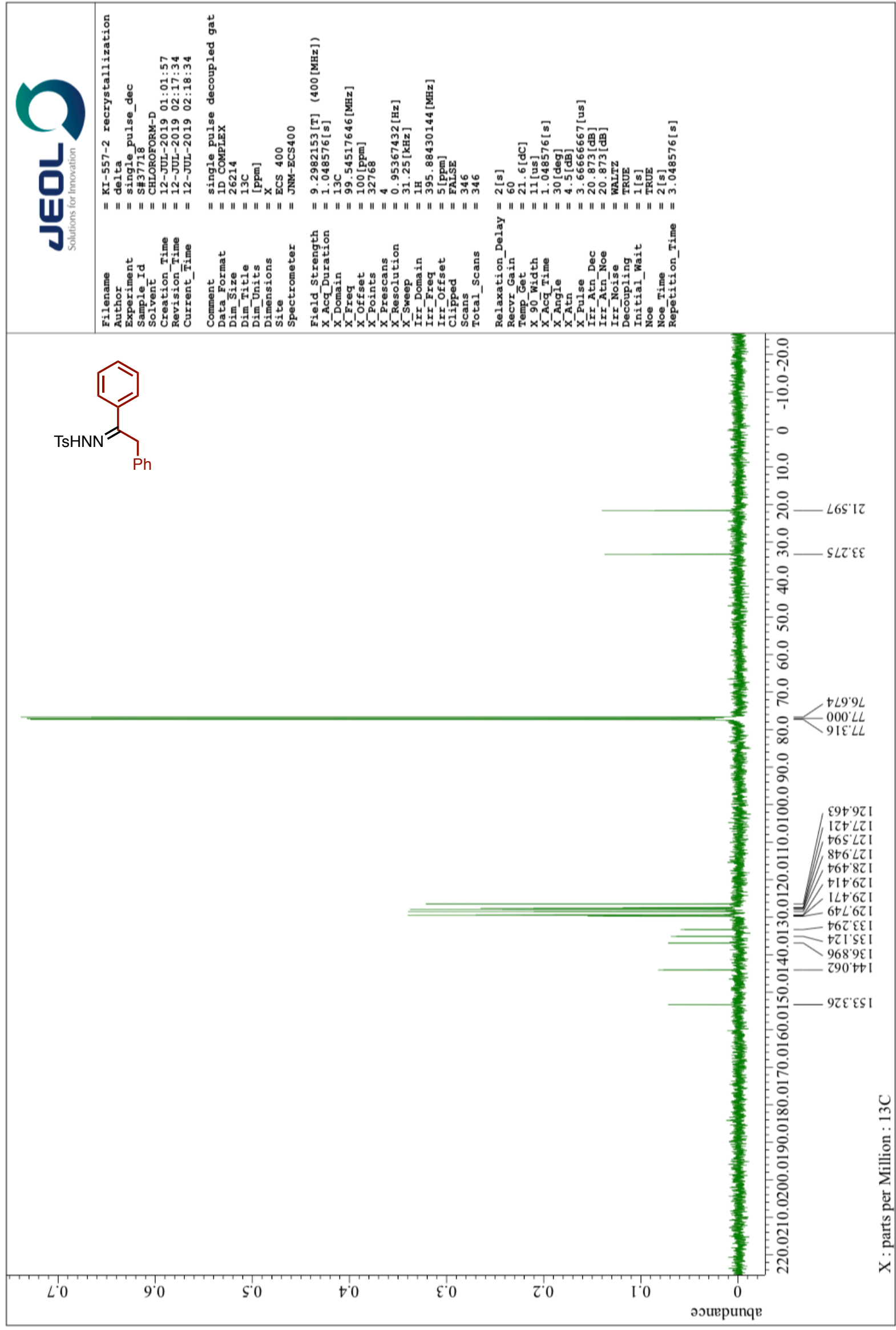


${ }^{1} \mathrm{H}$ NMR of $\mathbf{2 h}\left(400 \mathrm{MHz}, \mathrm{CDCl}_{3}\right)$

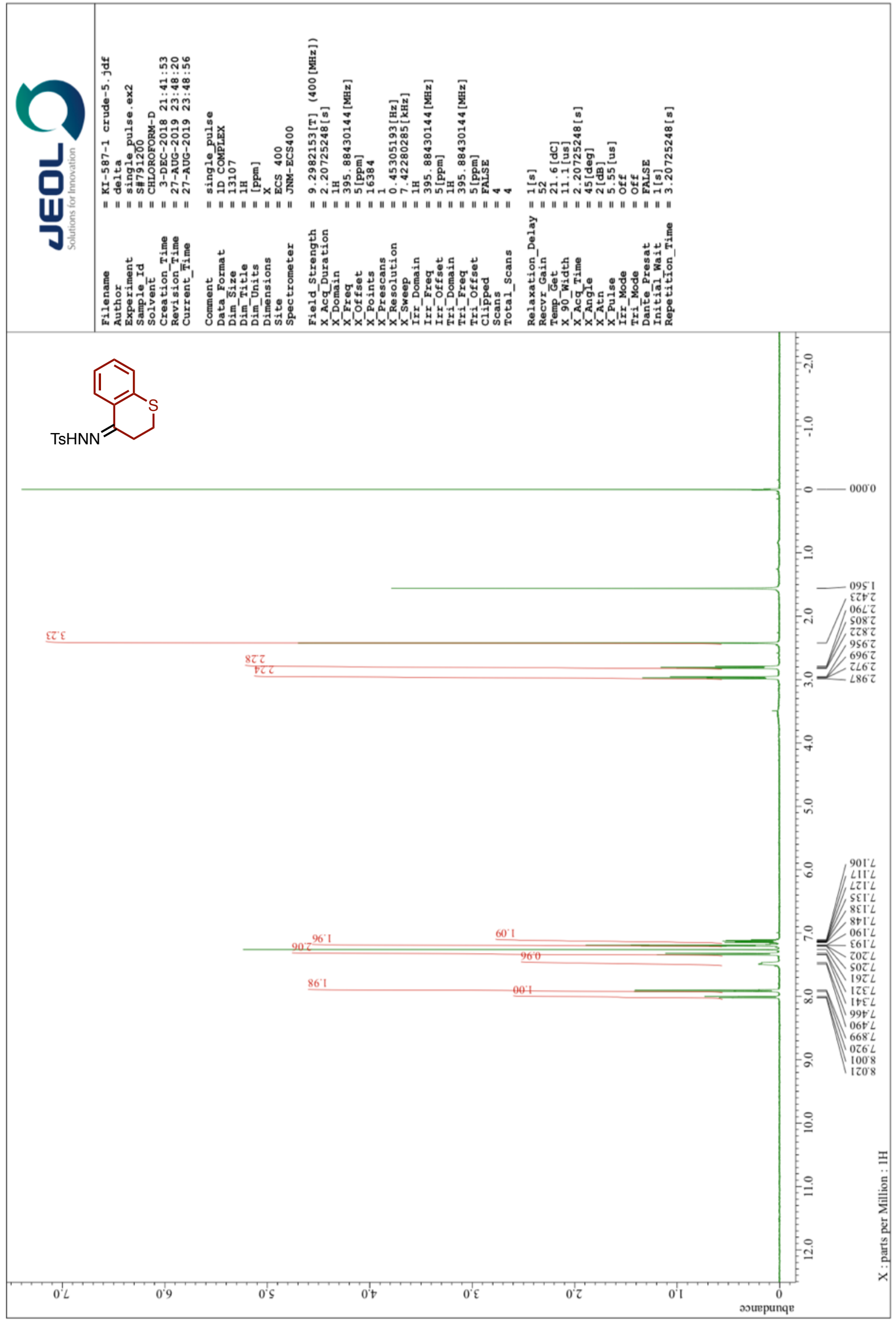


${ }^{13} \mathrm{C}$ NMR of $\mathbf{2 h}\left(101 \mathrm{MHz}, \mathrm{CDCl}_{3}\right)$

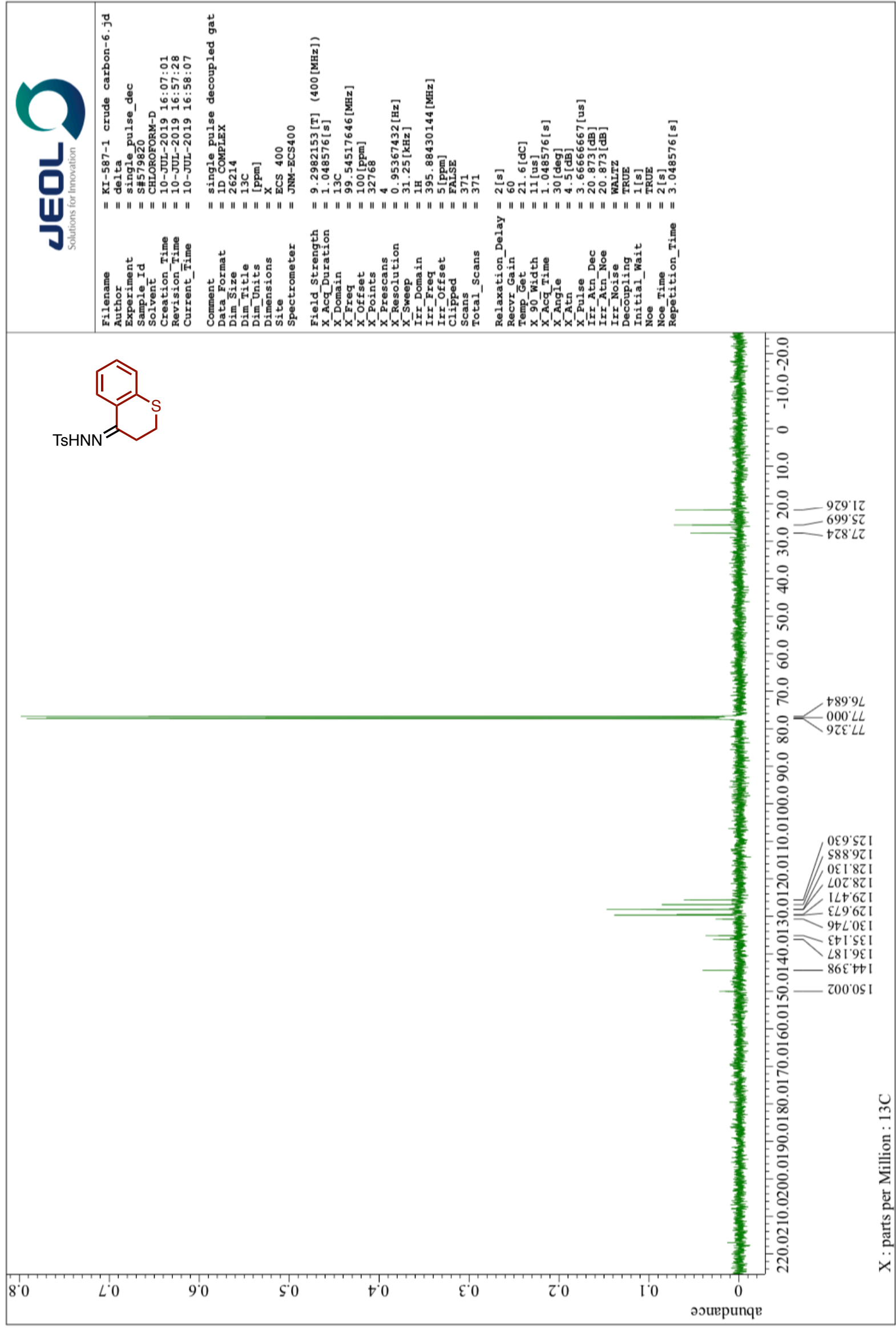


${ }^{1} \mathrm{H}$ NMR of $2 \mathbf{i}\left(400 \mathrm{MHz}, \mathrm{CDCl}_{3}\right)$

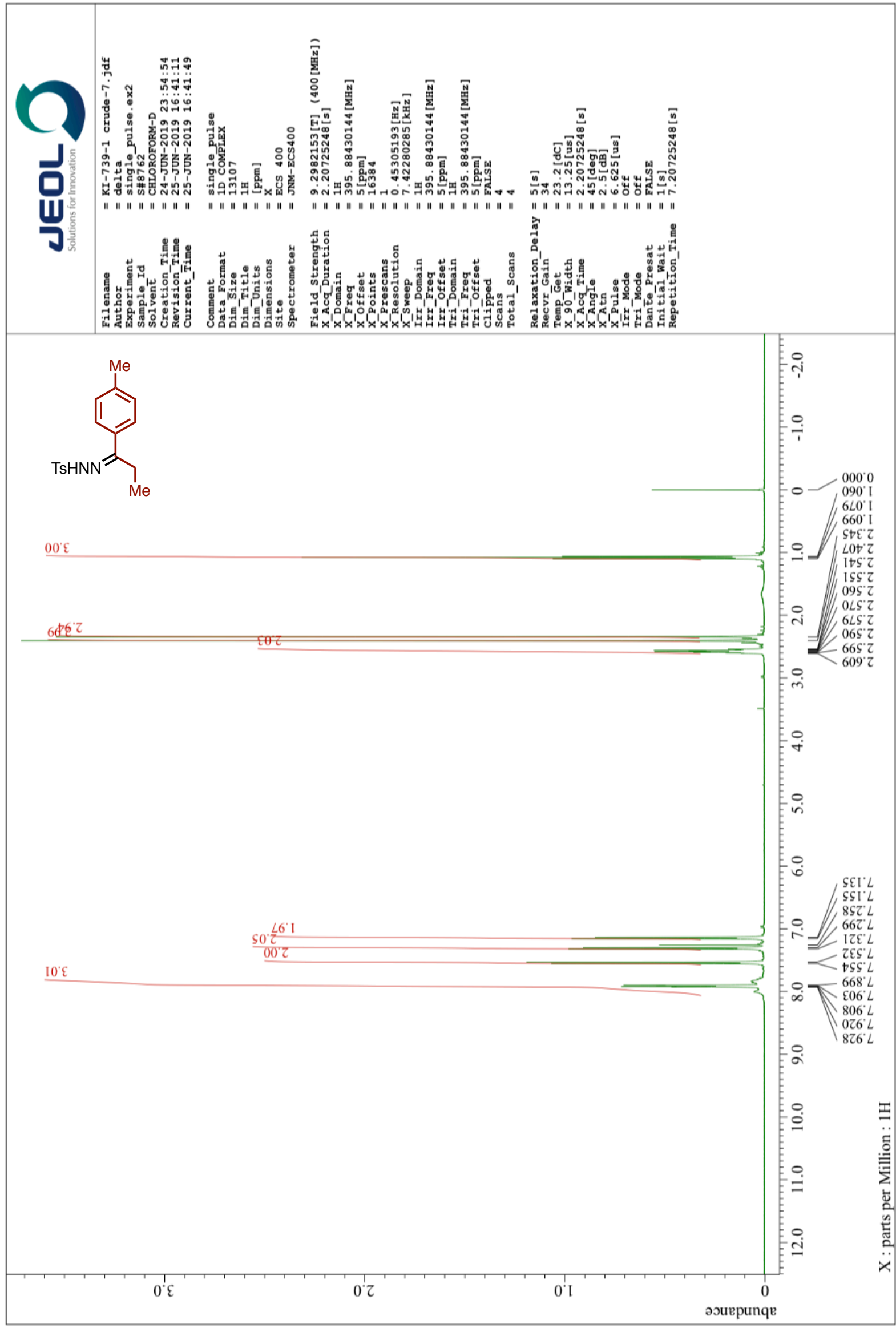


${ }^{13} \mathrm{C}$ NMR of $2 \mathbf{i}\left(101 \mathrm{MHz}, \mathrm{CDCl}_{3}\right)$

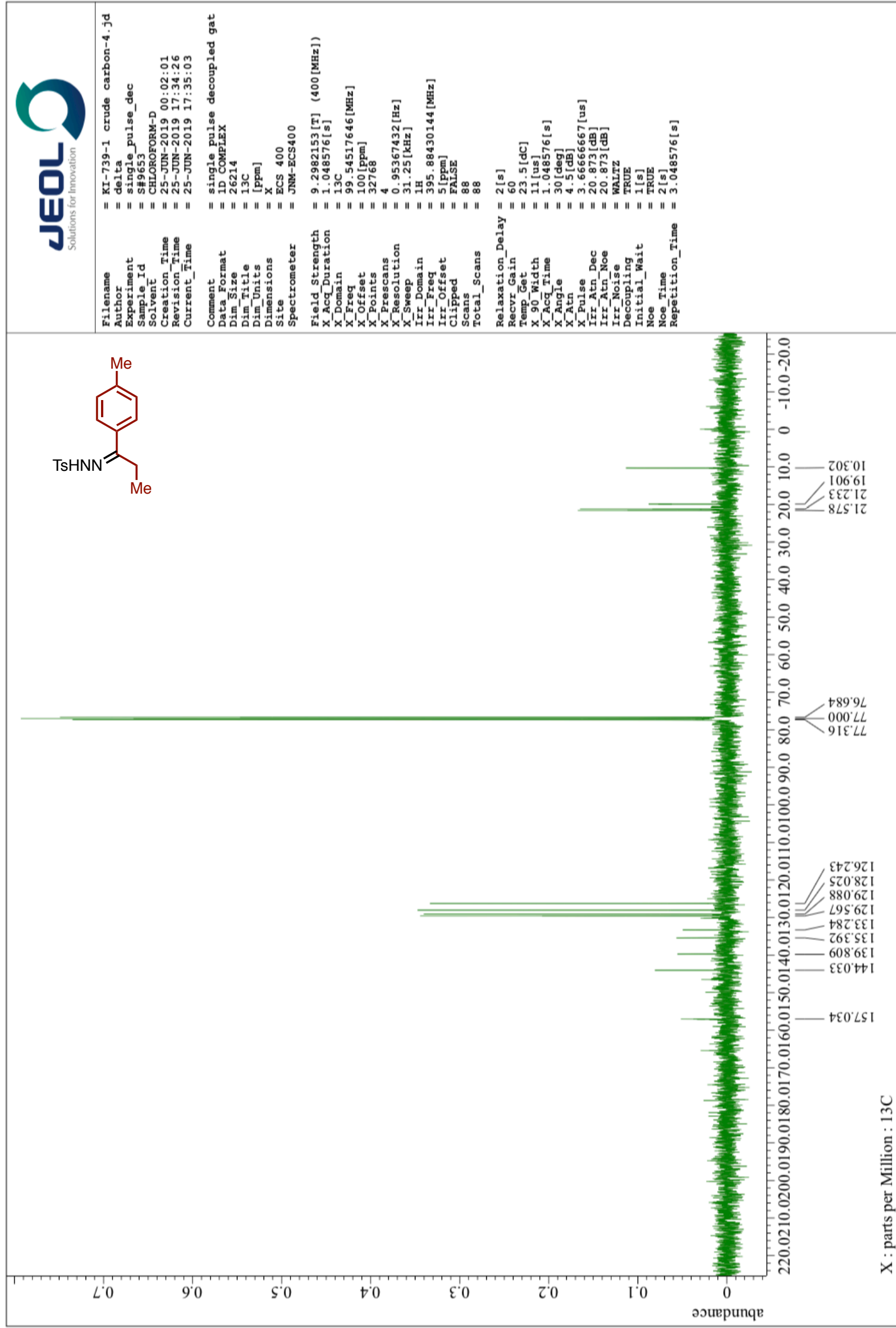


${ }^{1} \mathrm{H}$ NMR of $\mathbf{2 j}\left(400 \mathrm{MHz}, \mathrm{CDCl}_{3}\right.$ )

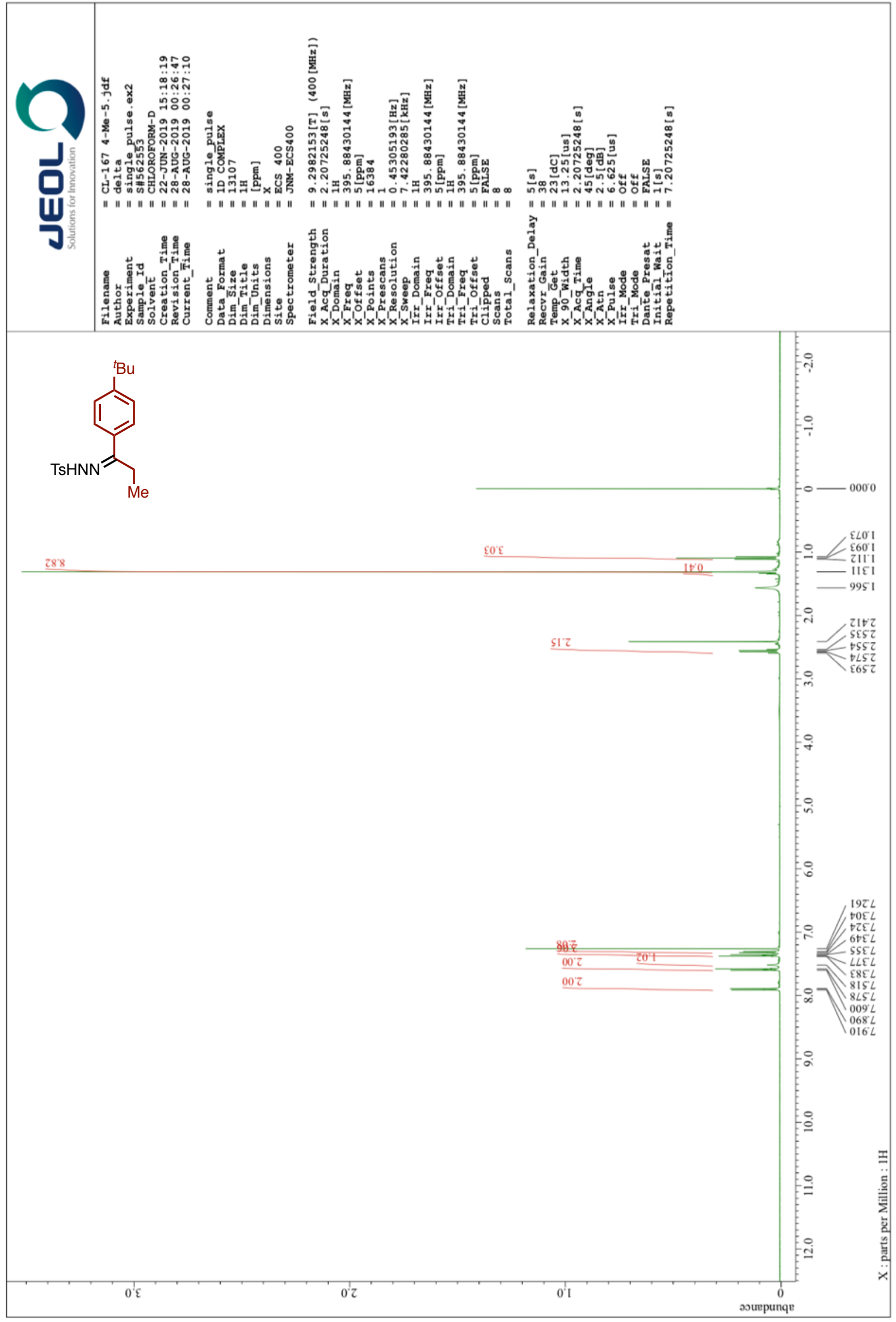


${ }^{13} \mathrm{C}$ NMR of $\mathbf{2 j}\left(101 \mathrm{MHz}, \mathrm{CDCl}_{3}\right)$

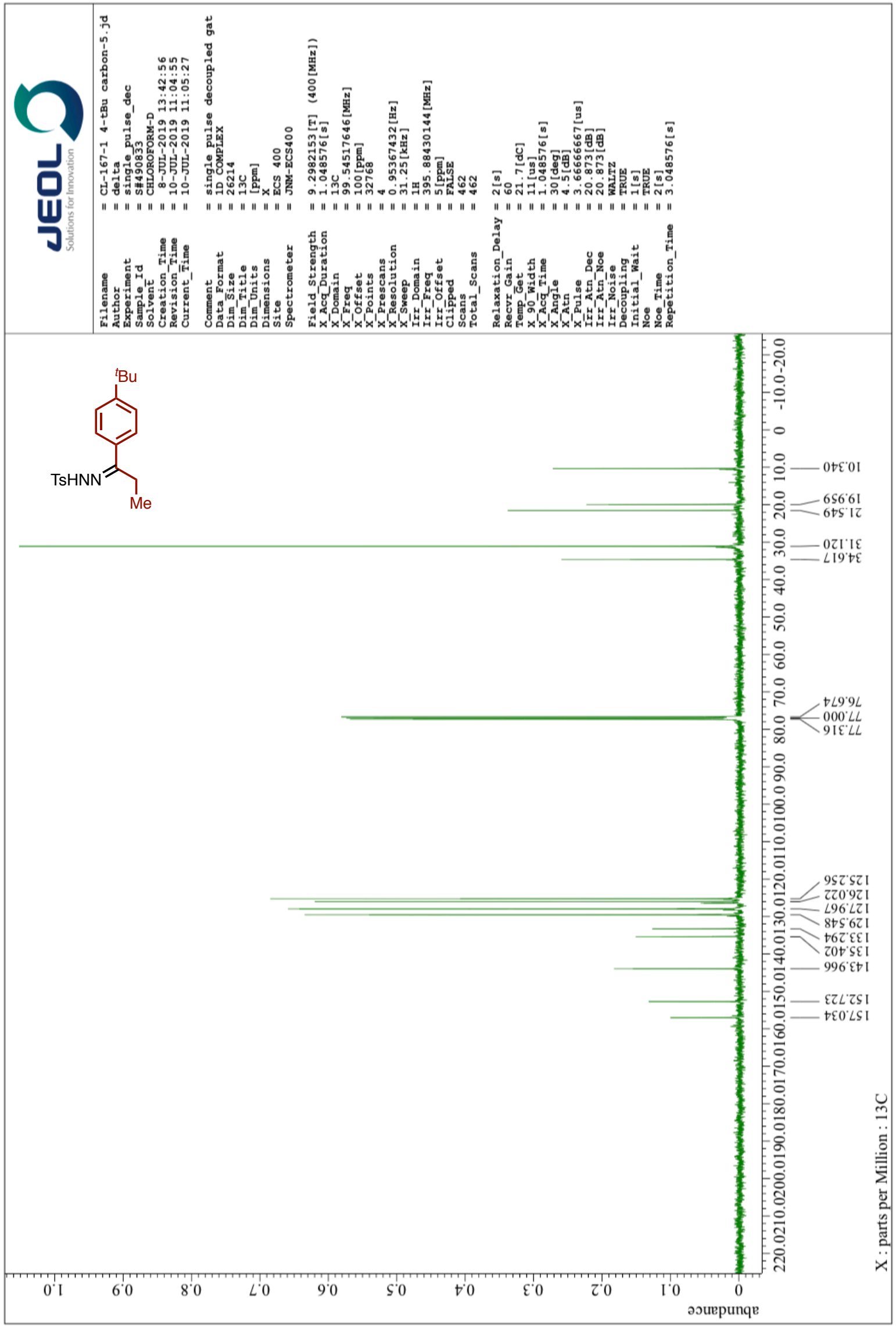


${ }^{1} \mathrm{H}$ NMR of $\mathbf{2 k}\left(400 \mathrm{MHz}, \mathrm{CDCl}_{3}\right)$

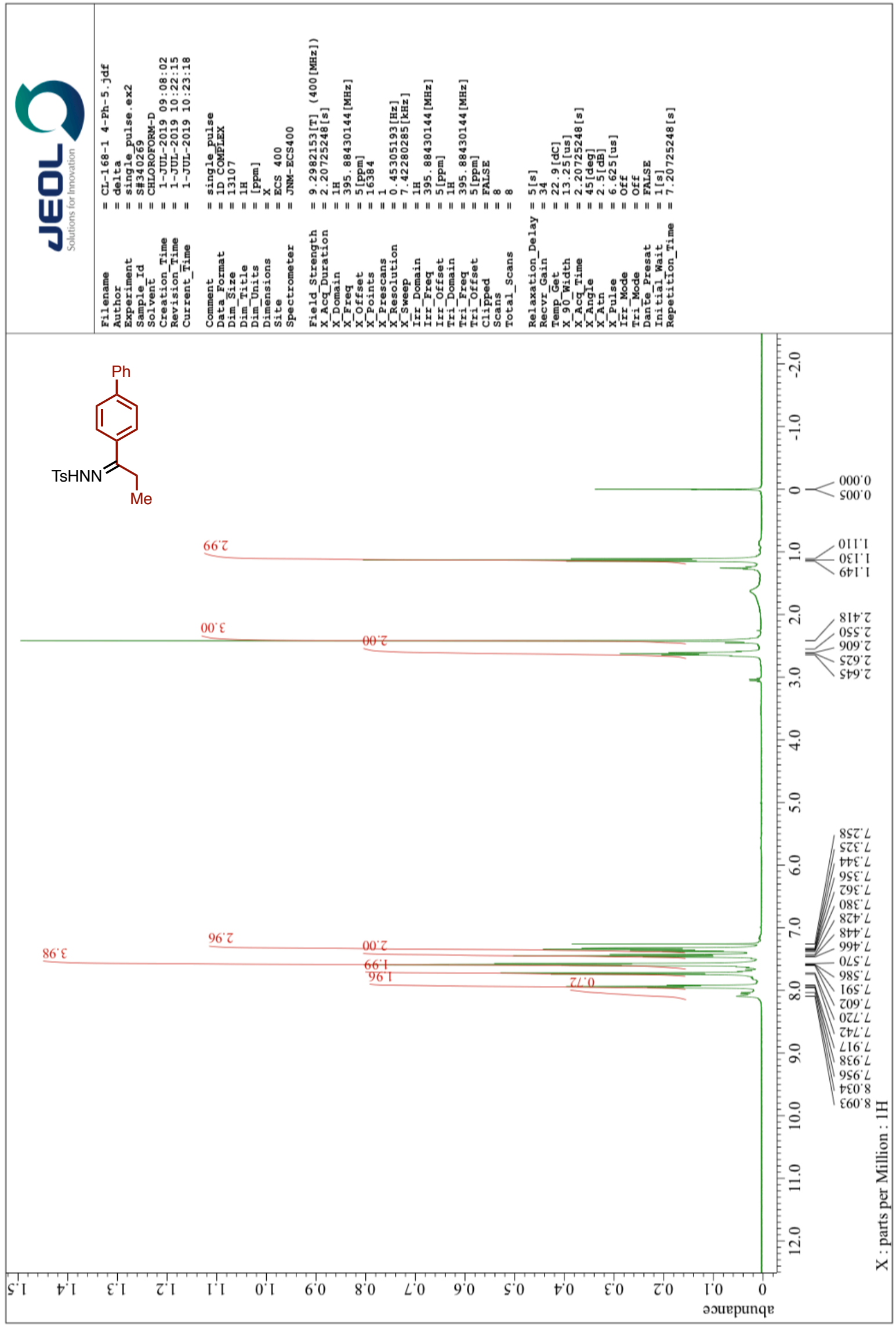


${ }^{13} \mathrm{C}$ NMR of $\mathbf{2 k}\left(101 \mathrm{MHz}, \mathrm{CDCl}_{3}\right)$

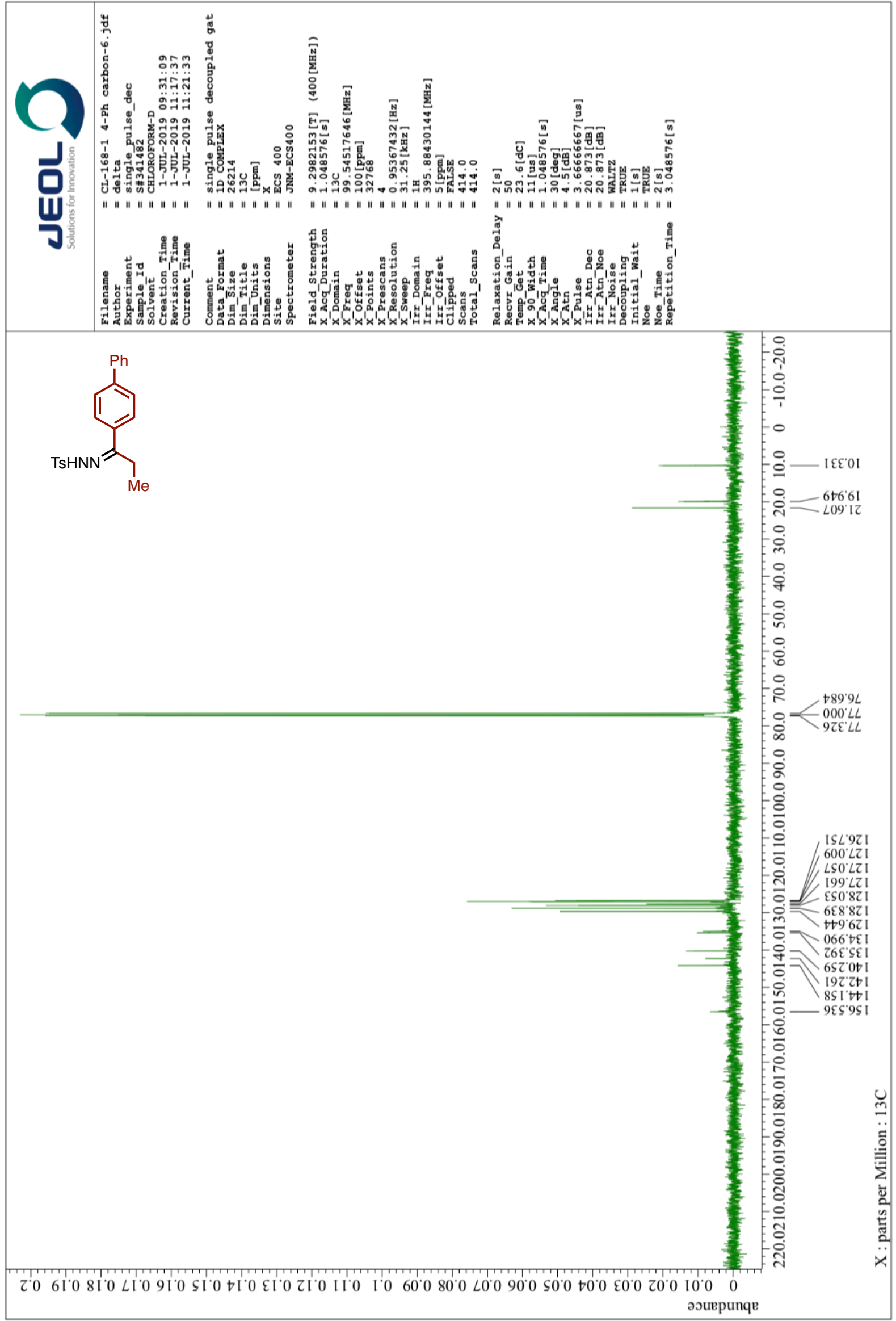


${ }^{1} \mathrm{H}$ NMR of $21\left(400 \mathrm{MHz}, \mathrm{CDCl}_{3}\right)$

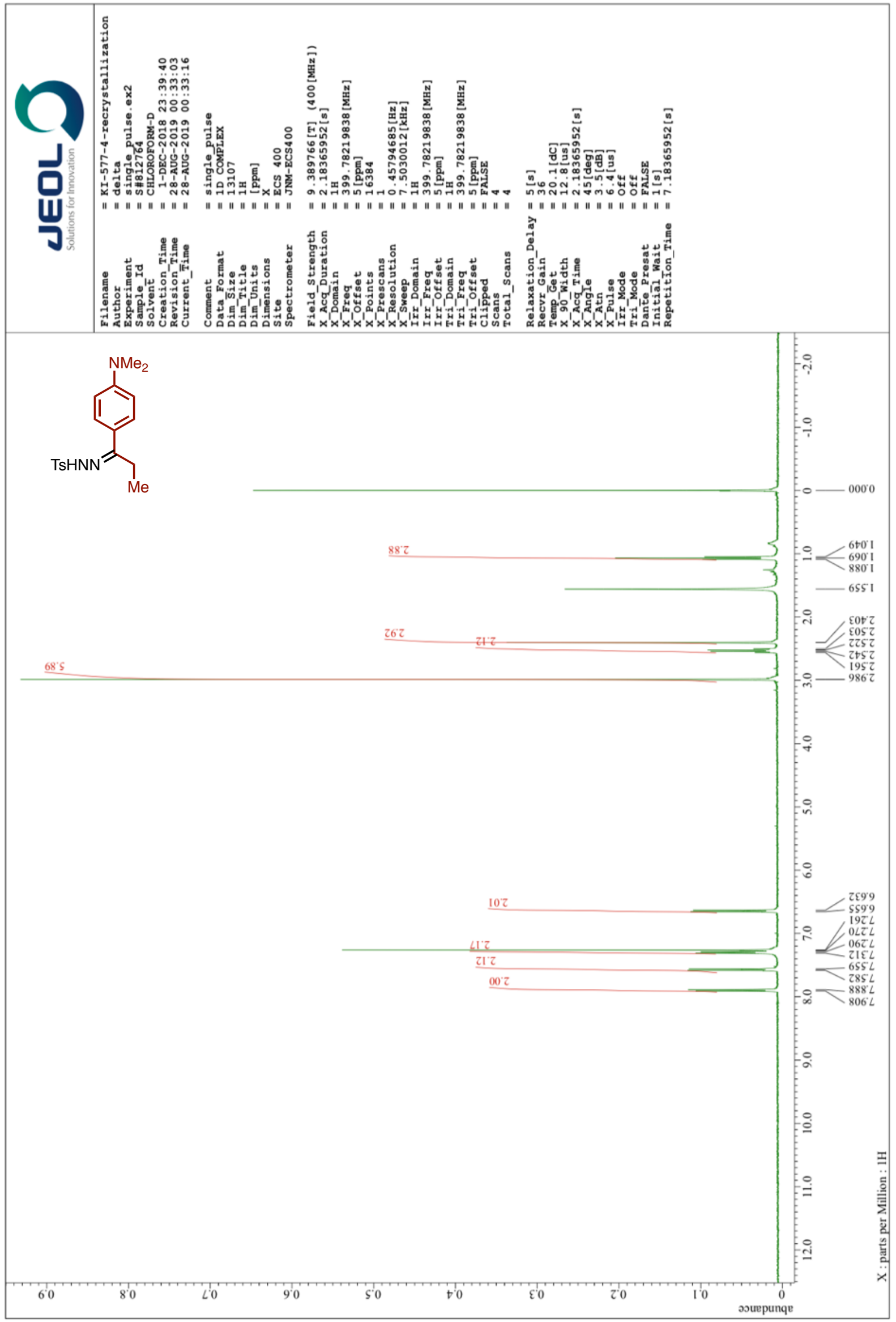


${ }^{13} \mathrm{C}$ NMR of $2 \mathbf{l}\left(101 \mathrm{MHz}, \mathrm{CDCl}_{3}\right)$

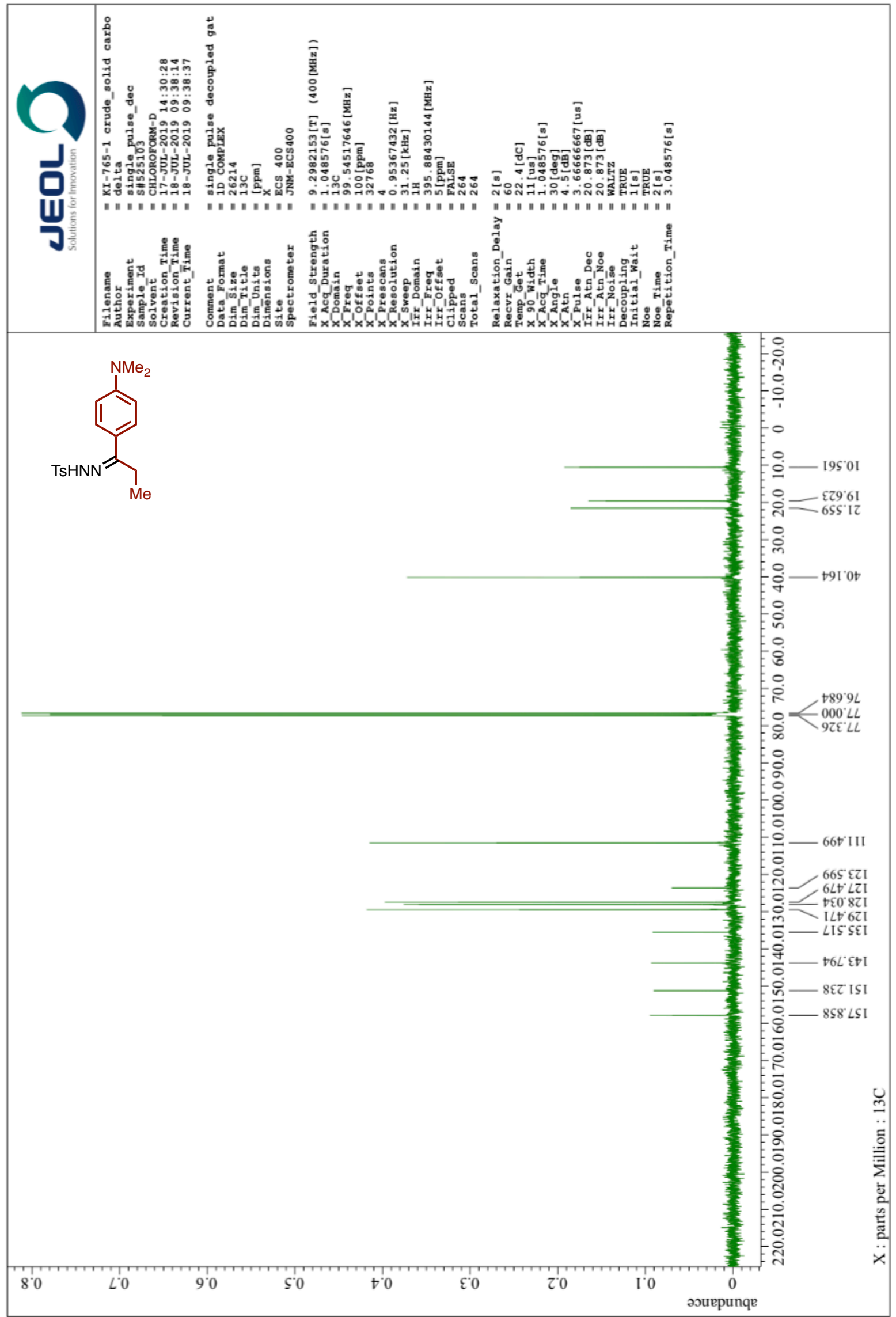


${ }^{1} \mathrm{H} \mathrm{NMR}$ of $2 \mathbf{n}\left(400 \mathrm{MHz}, \mathrm{CDCl}_{3}\right)$

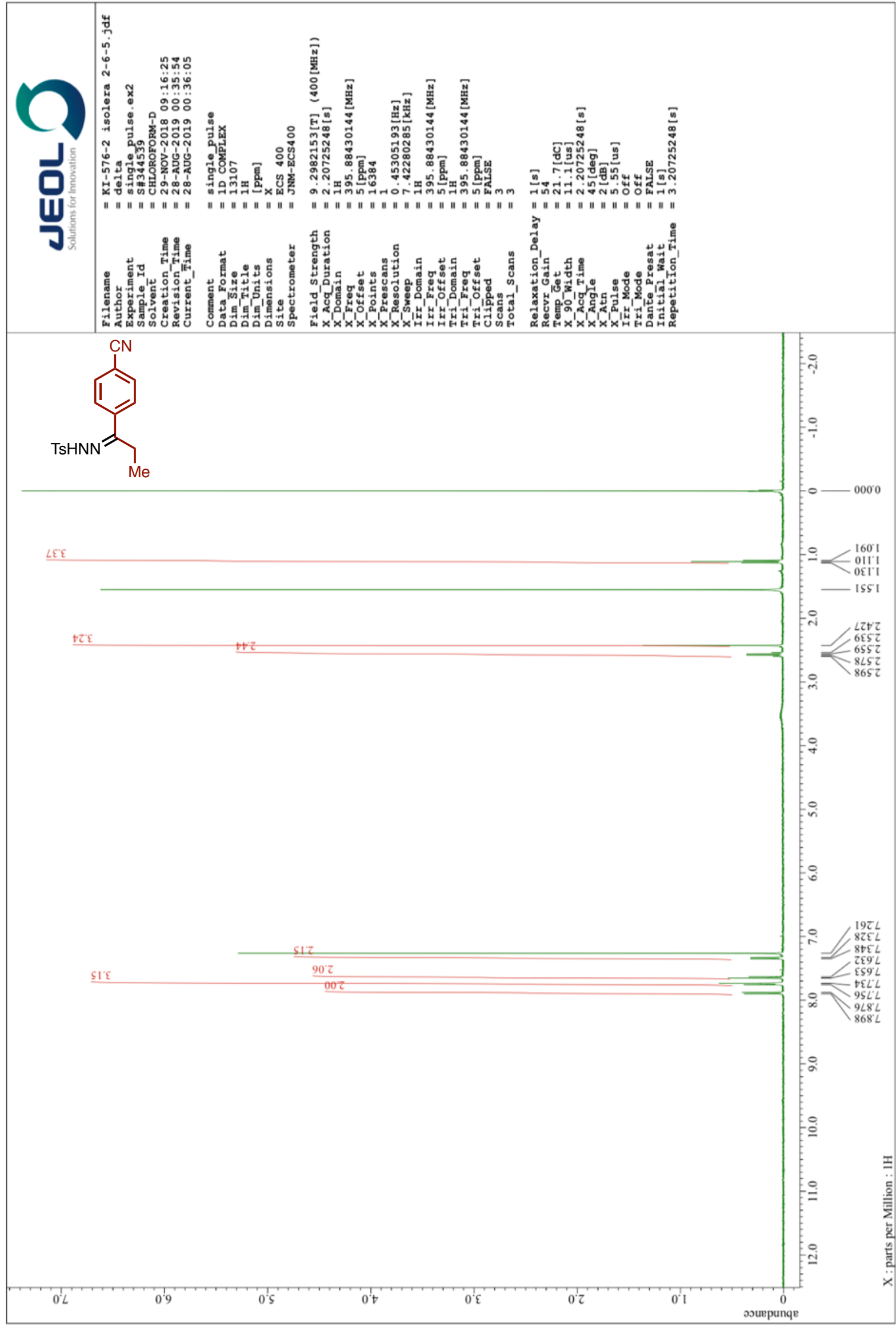


${ }^{13} \mathrm{C}$ NMR of 2 n $\left(101 \mathrm{MHz}, \mathrm{CDCl}_{3}\right)$

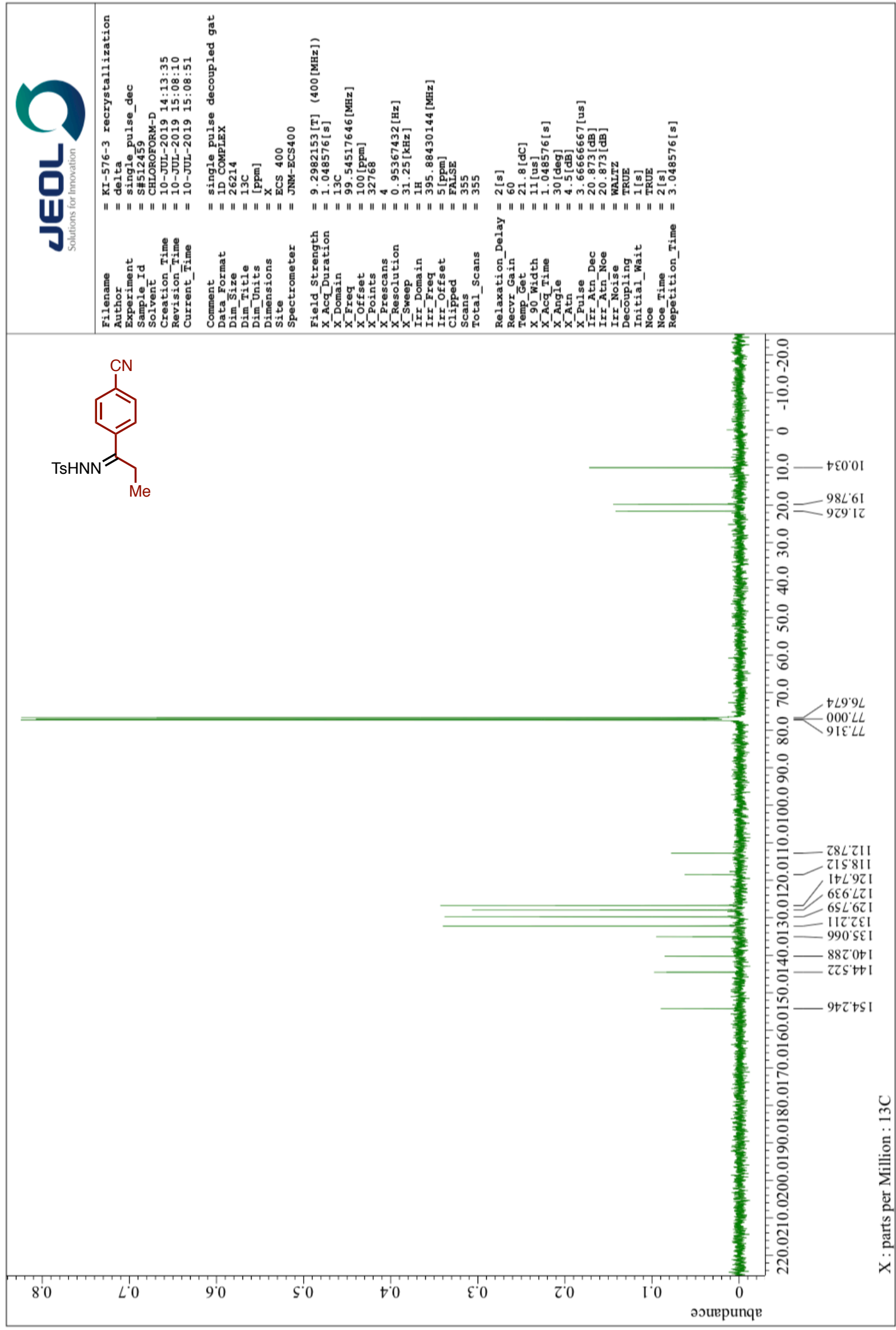


${ }^{1} \mathrm{H} \mathrm{NMR}$ of $2 \mathrm{o}\left(400 \mathrm{MHz}, \mathrm{CDCl}_{3}\right)$

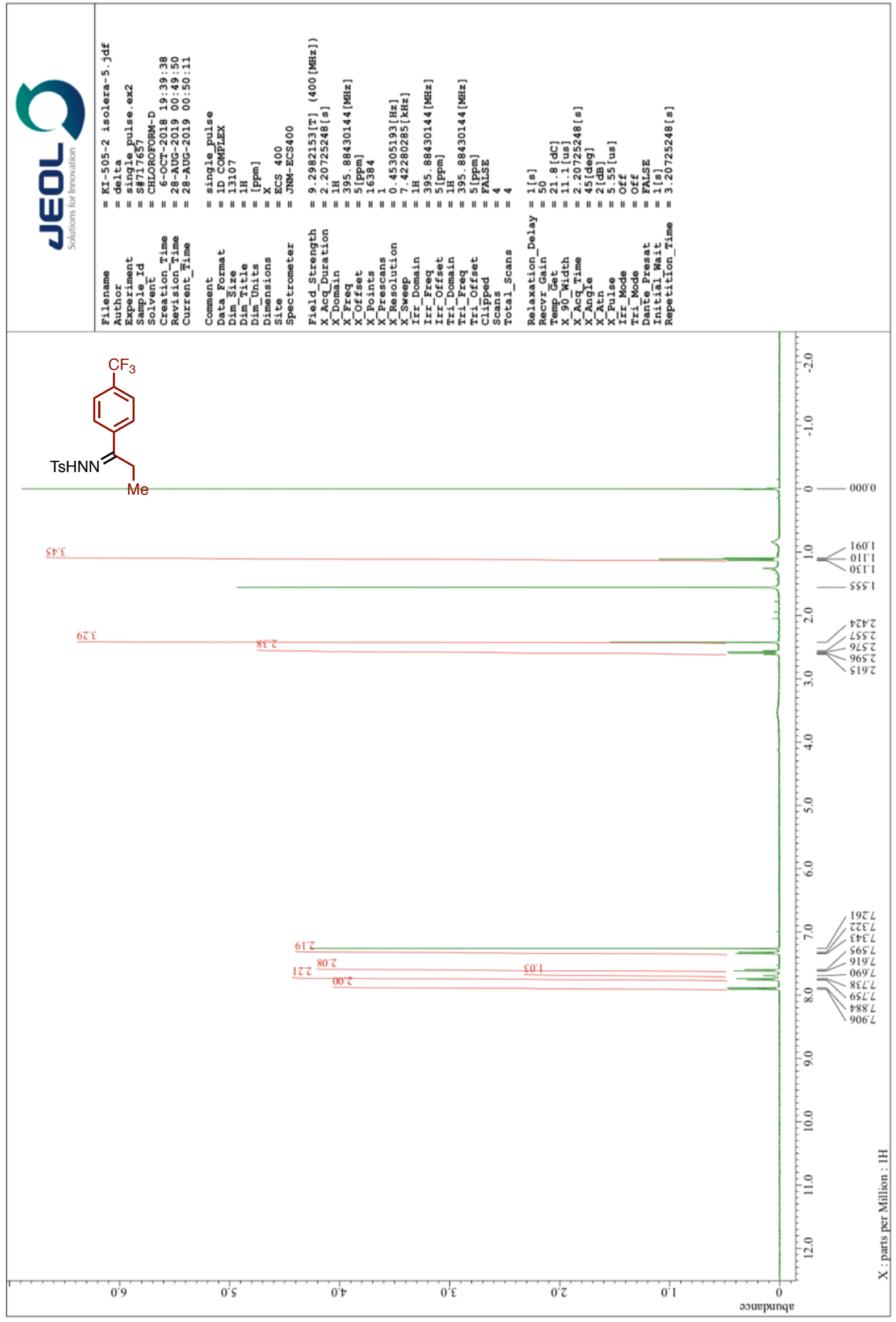


${ }^{13} \mathrm{C}$ NMR of $20\left(101 \mathrm{MHz}, \mathrm{CDCl}_{3}\right)$

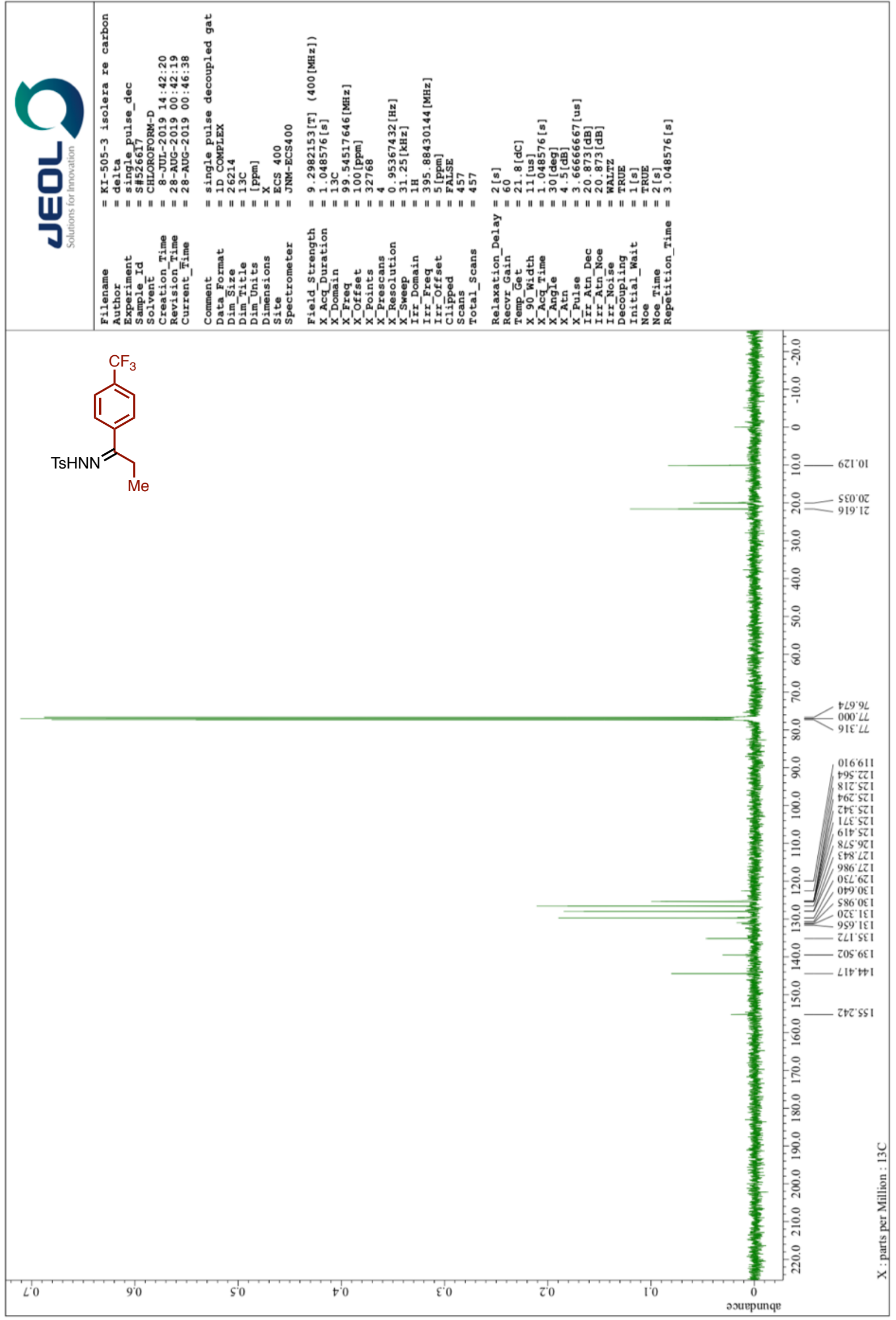


${ }^{1} \mathrm{H}$ NMR of $\mathbf{2 p}\left(400 \mathrm{MHz}, \mathrm{CDCl}_{3}\right.$ )

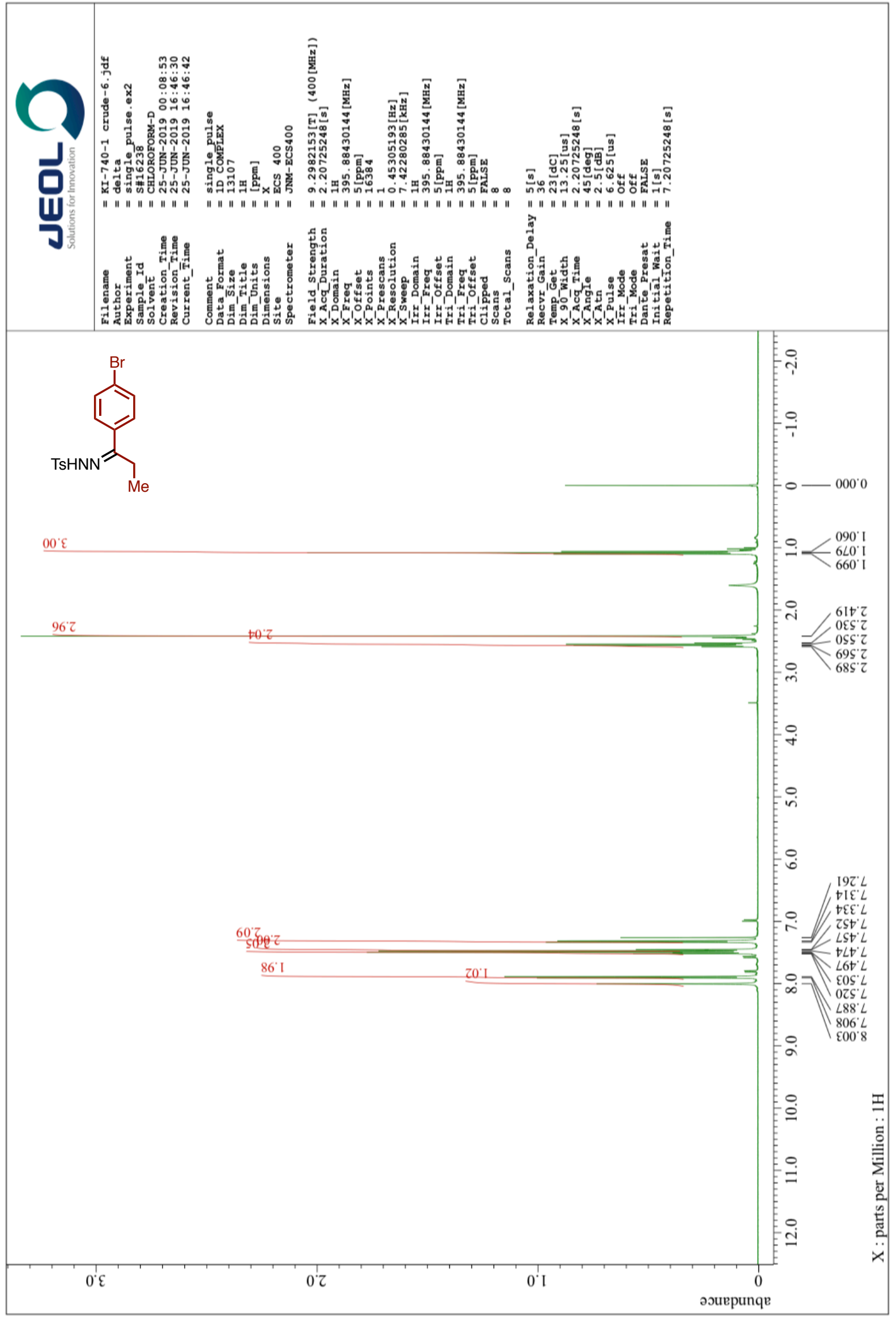


${ }^{13} \mathrm{C}$ NMR of $2 \mathbf{p}\left(101 \mathrm{MHz}, \mathrm{CDCl}_{3}\right)$

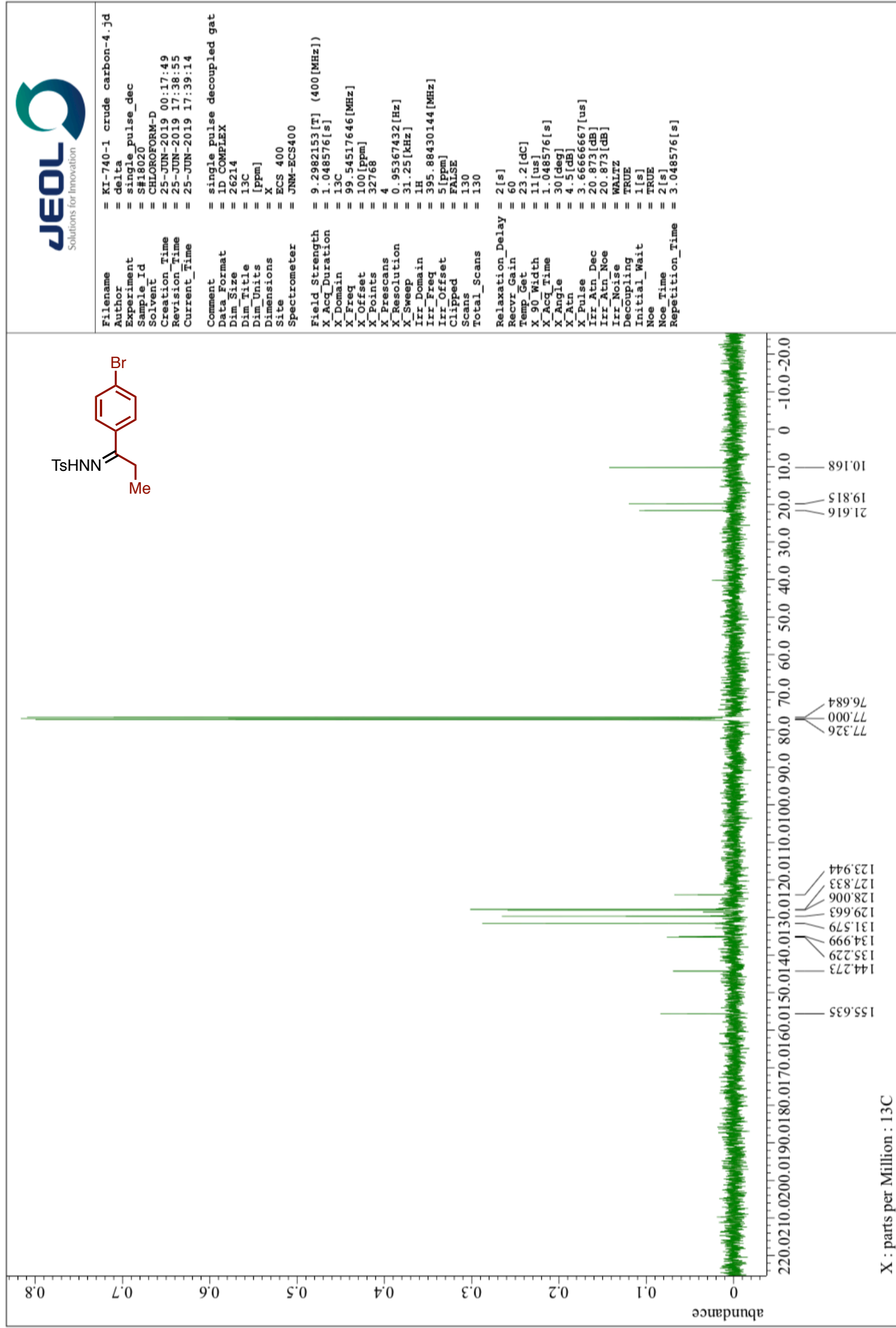


${ }^{1} \mathrm{H} \mathrm{NMR}$ of $\mathbf{2 q}\left(400 \mathrm{MHz}, \mathrm{CDCl}_{3}\right)$

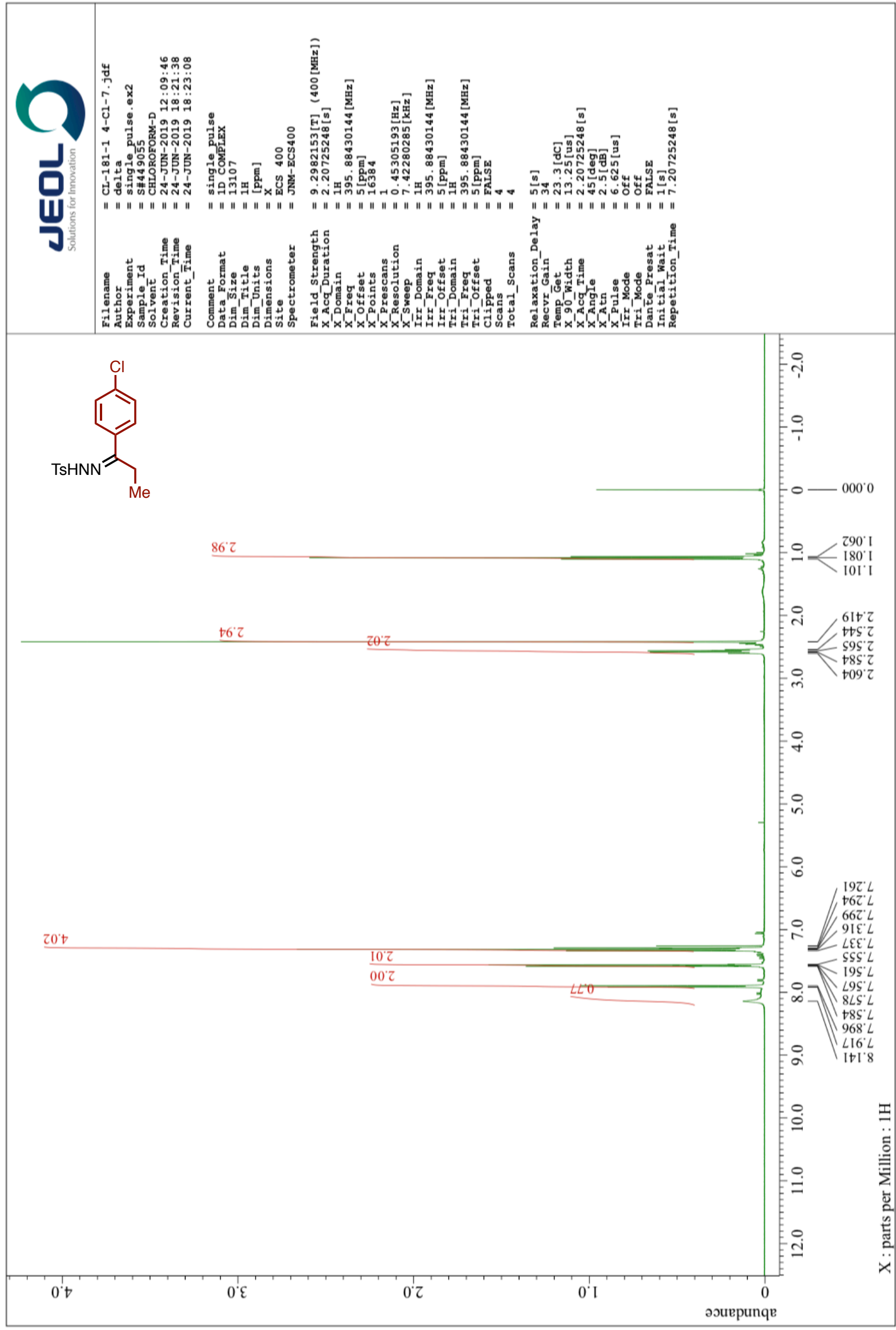


${ }^{13} \mathrm{C}$ NMR of $\mathbf{2 q}\left(101 \mathrm{MHz}, \mathrm{CDCl}_{3}\right)$

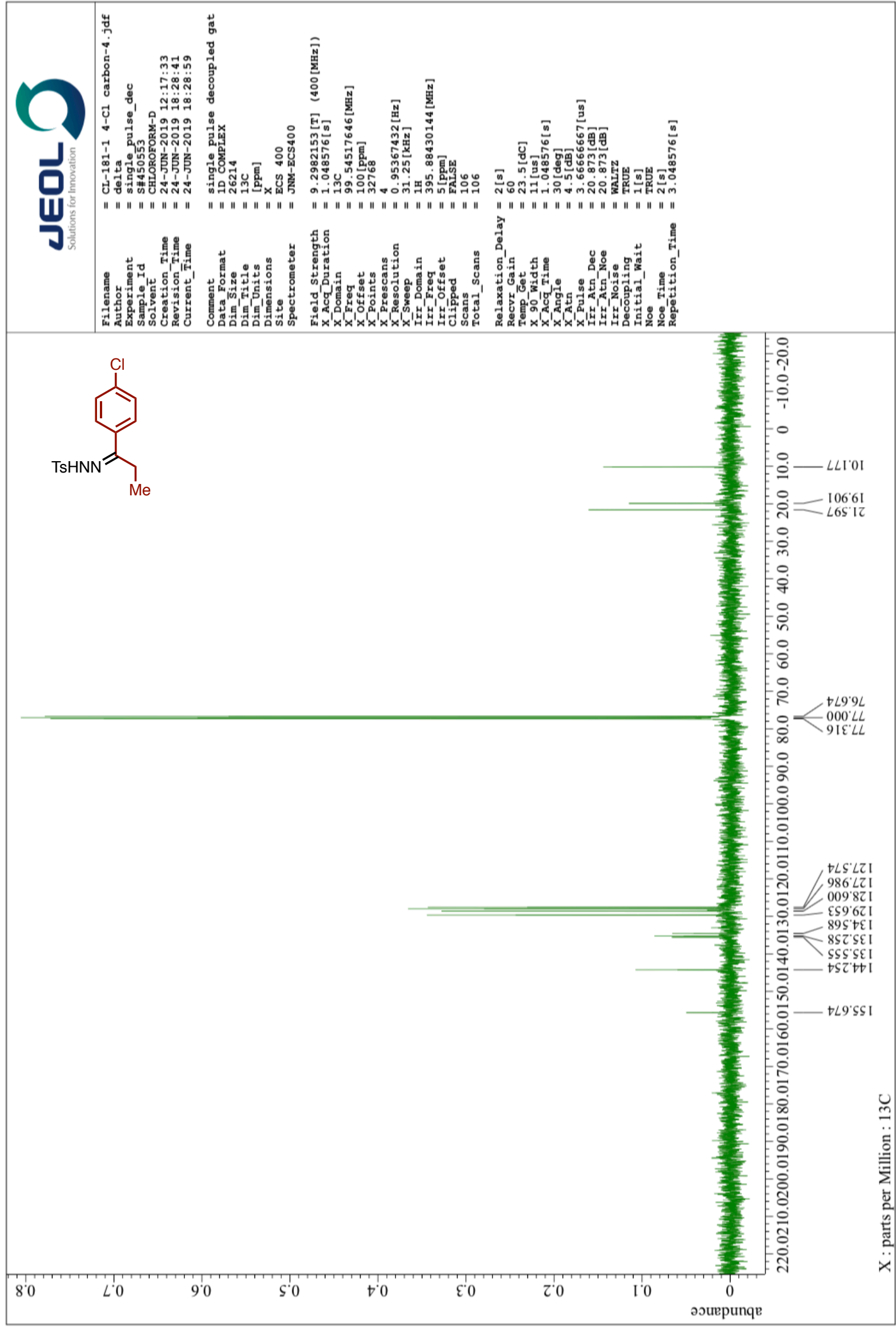


${ }^{1} \mathrm{H}$ NMR of $2 \mathbf{r}\left(400 \mathrm{MHz}, \mathrm{CDCl}_{3}\right)$

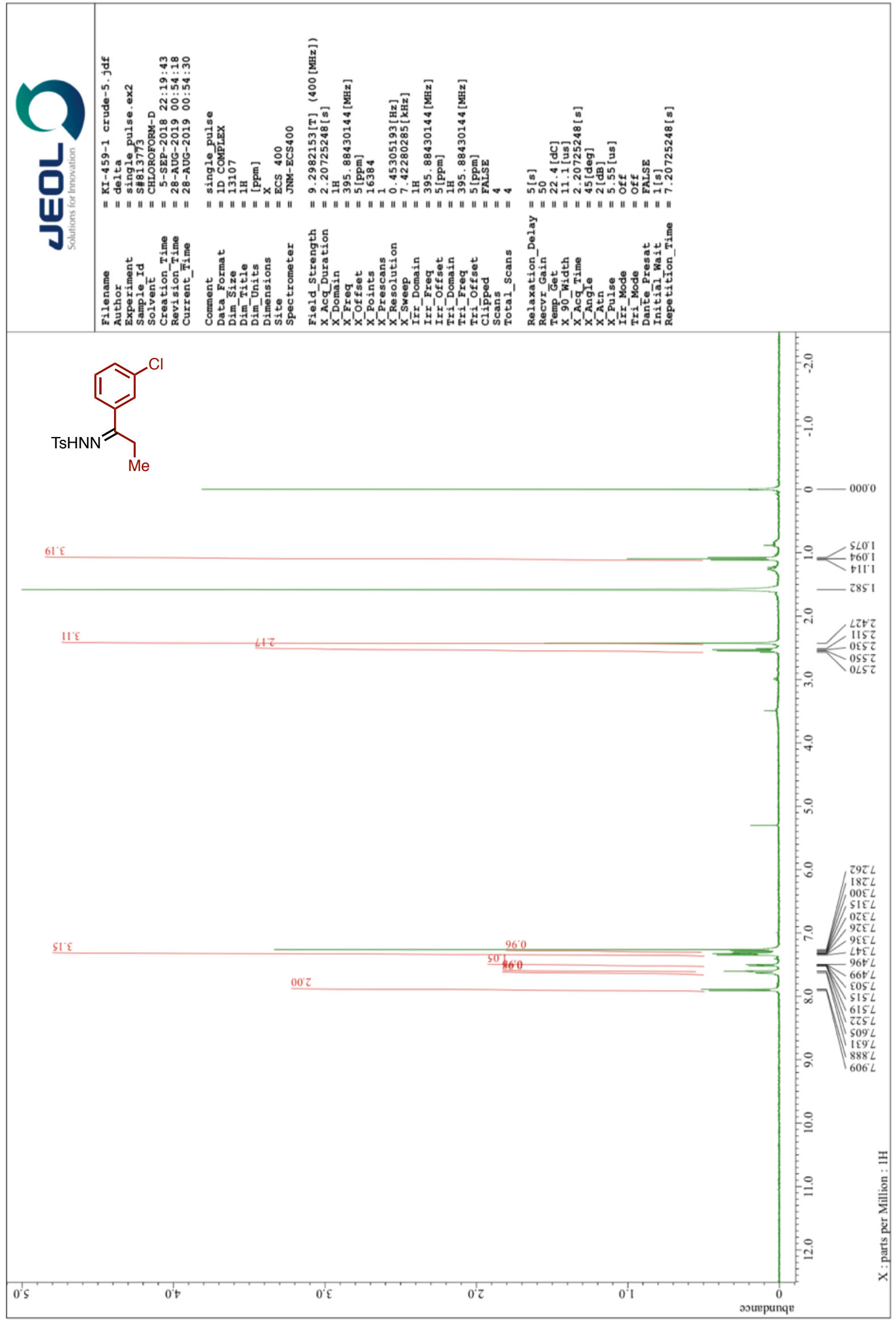


${ }^{13} \mathrm{C}$ NMR of $2 \mathbf{r}\left(101 \mathrm{MHz}, \mathrm{CDCl}_{3}\right)$

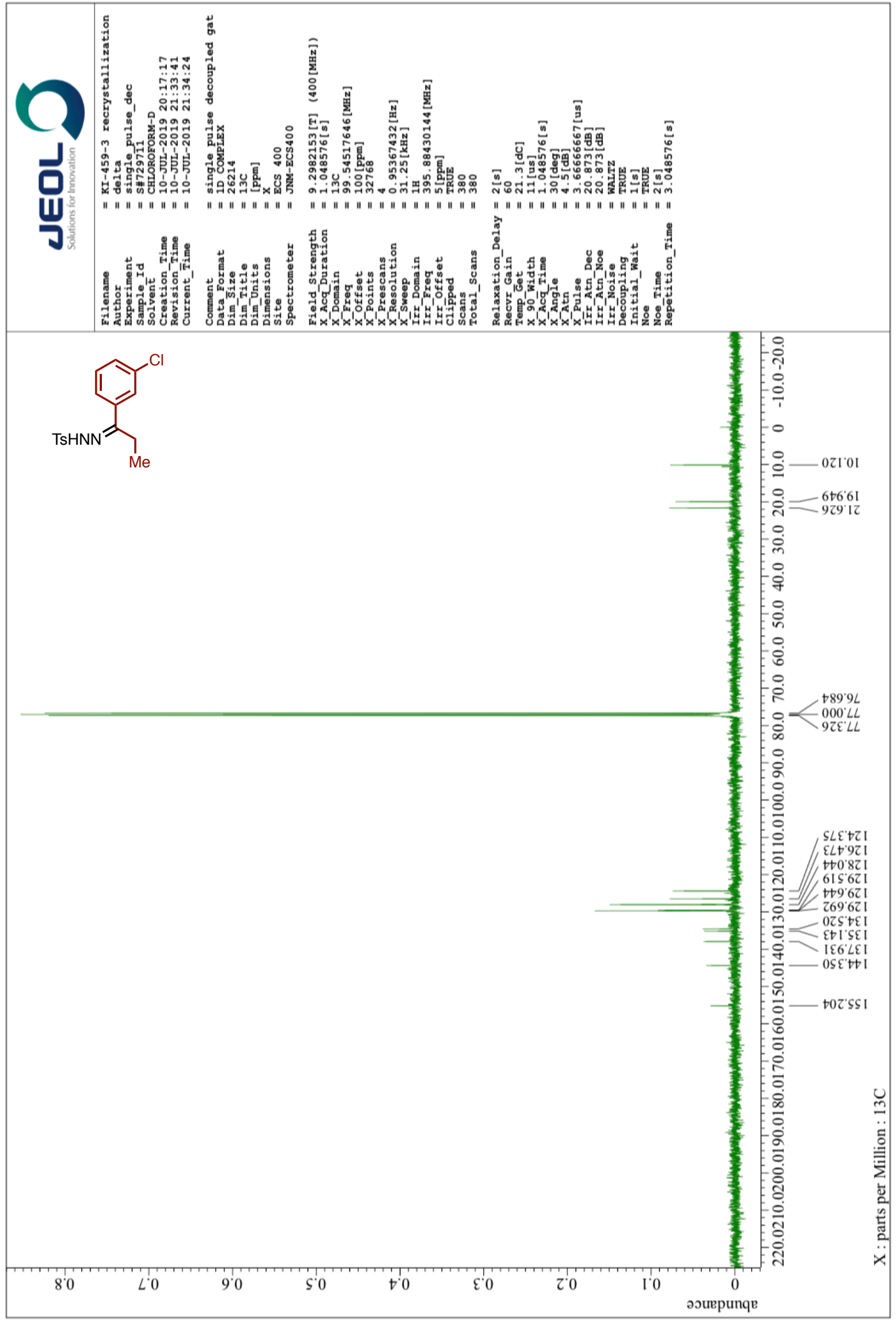


${ }^{1} \mathrm{H}$ NMR of $2 \mathbf{s}\left(400 \mathrm{MHz}, \mathrm{CDCl}_{3}\right)$

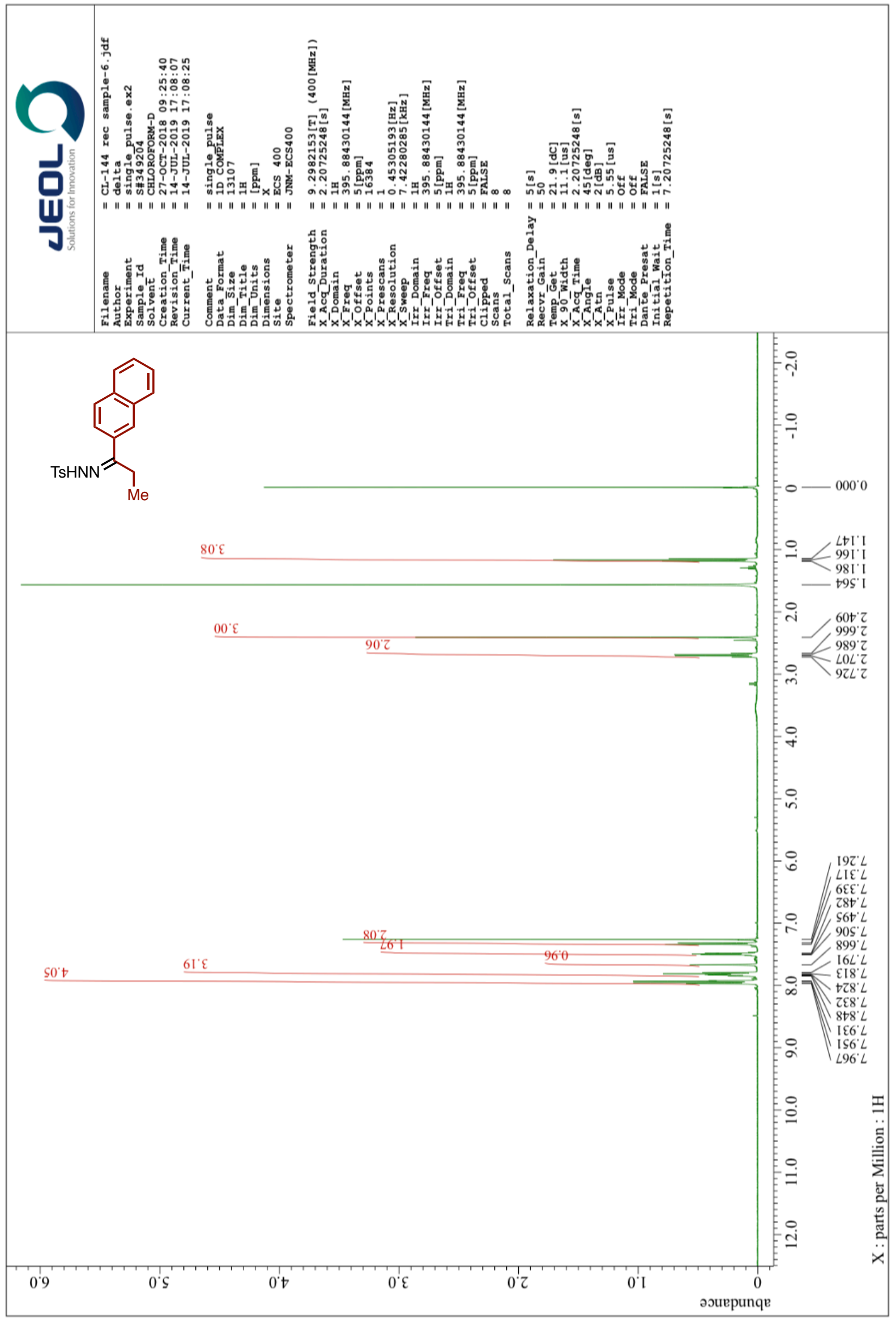


${ }^{13} \mathrm{C}$ NMR of $2 \mathbf{s}\left(101 \mathrm{MHz}, \mathrm{CDCl}_{3}\right)$

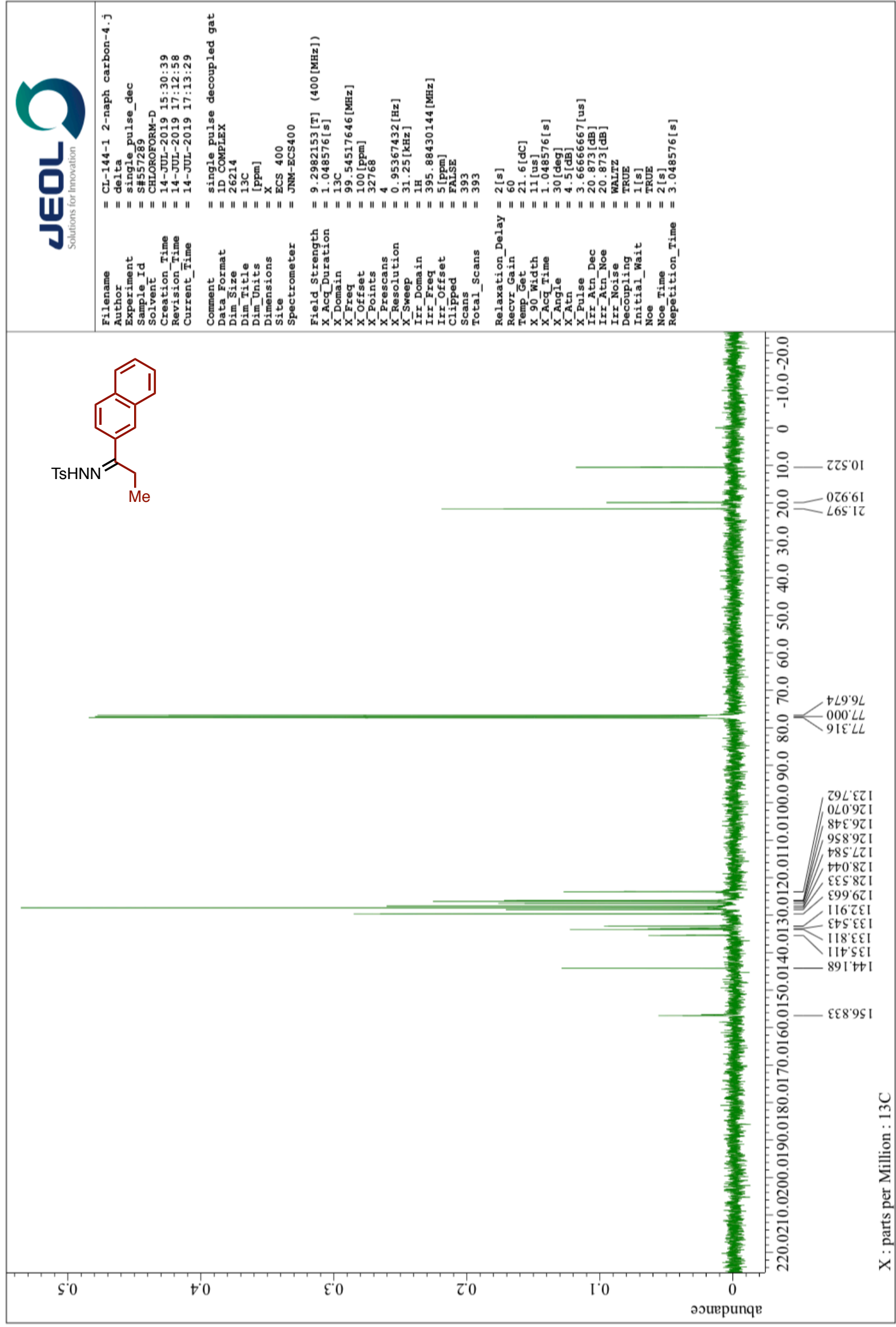


${ }^{1} \mathrm{H}$ NMR of $2 t\left(400 \mathrm{MHz}, \mathrm{CDCl}_{3}\right)$

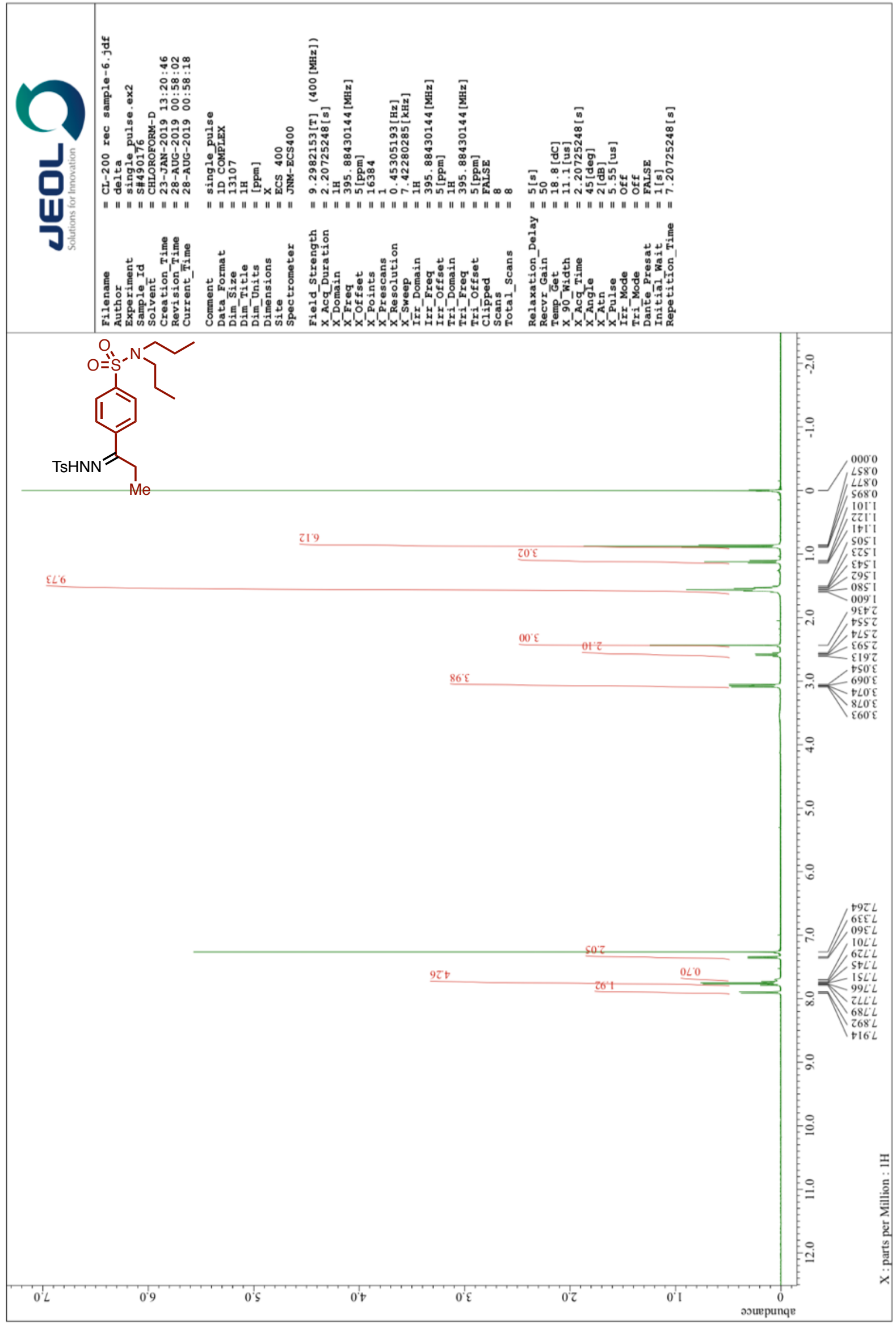


${ }^{13} \mathrm{C}$ NMR of $2 \mathbf{t}\left(101 \mathrm{MHz}, \mathrm{CDCl}_{3}\right)$

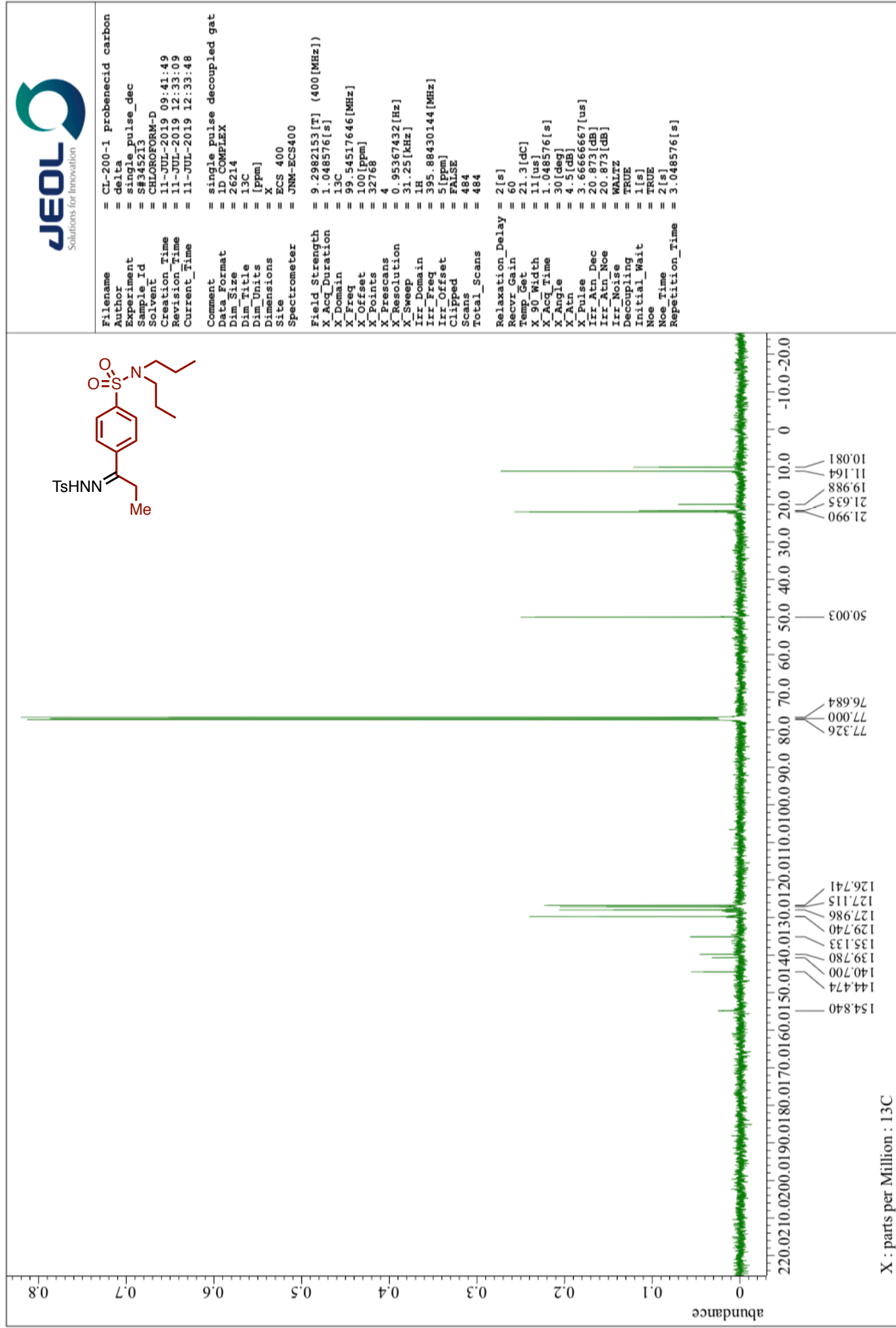


${ }^{1} \mathrm{H}$ NMR of $6\left(400 \mathrm{MHz}, \mathrm{CDCl}_{3}\right)$

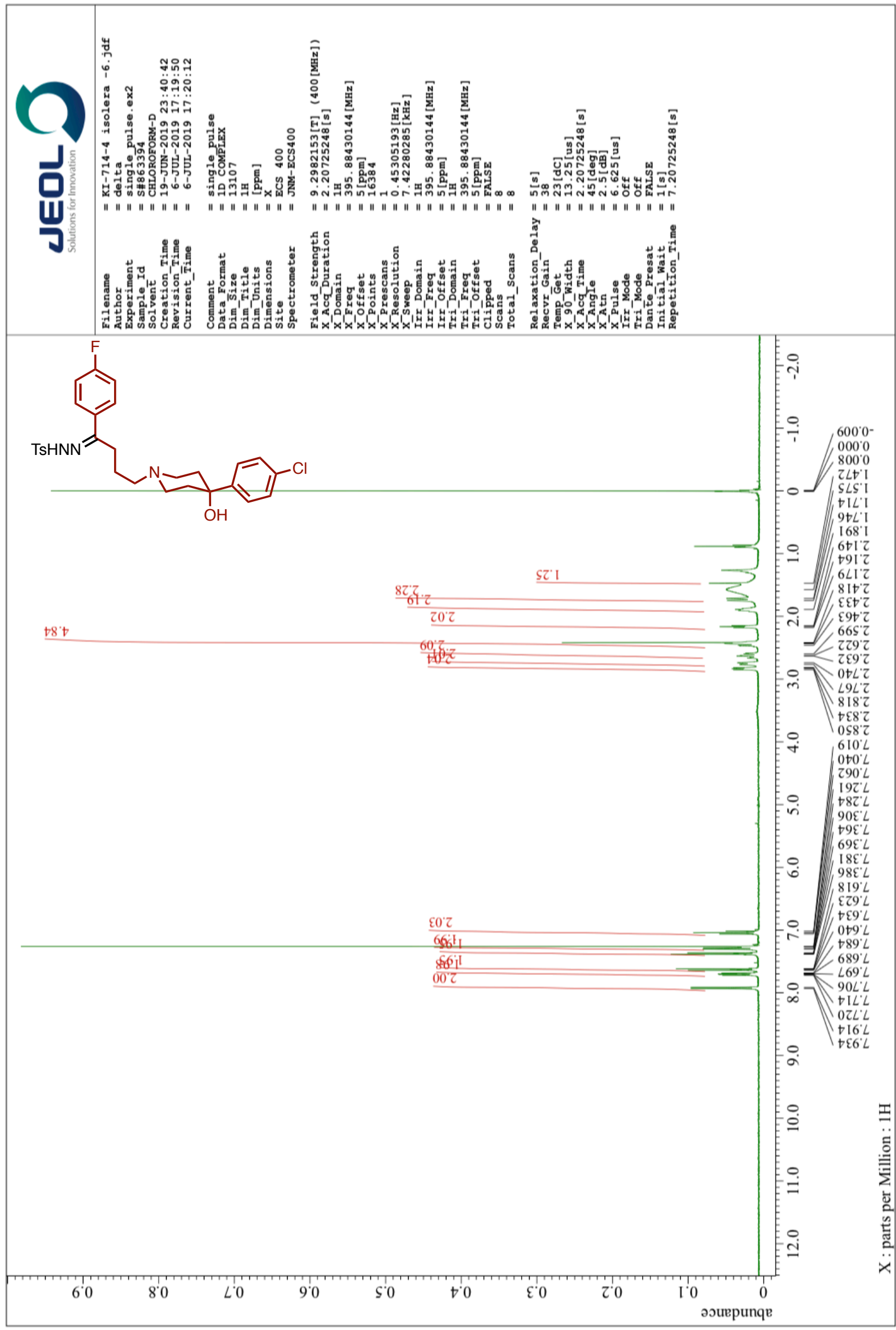


${ }^{13} \mathrm{C}$ NMR of $\mathbf{2 j}\left(101 \mathrm{MHz}, \mathrm{CDCl}_{3}\right)$

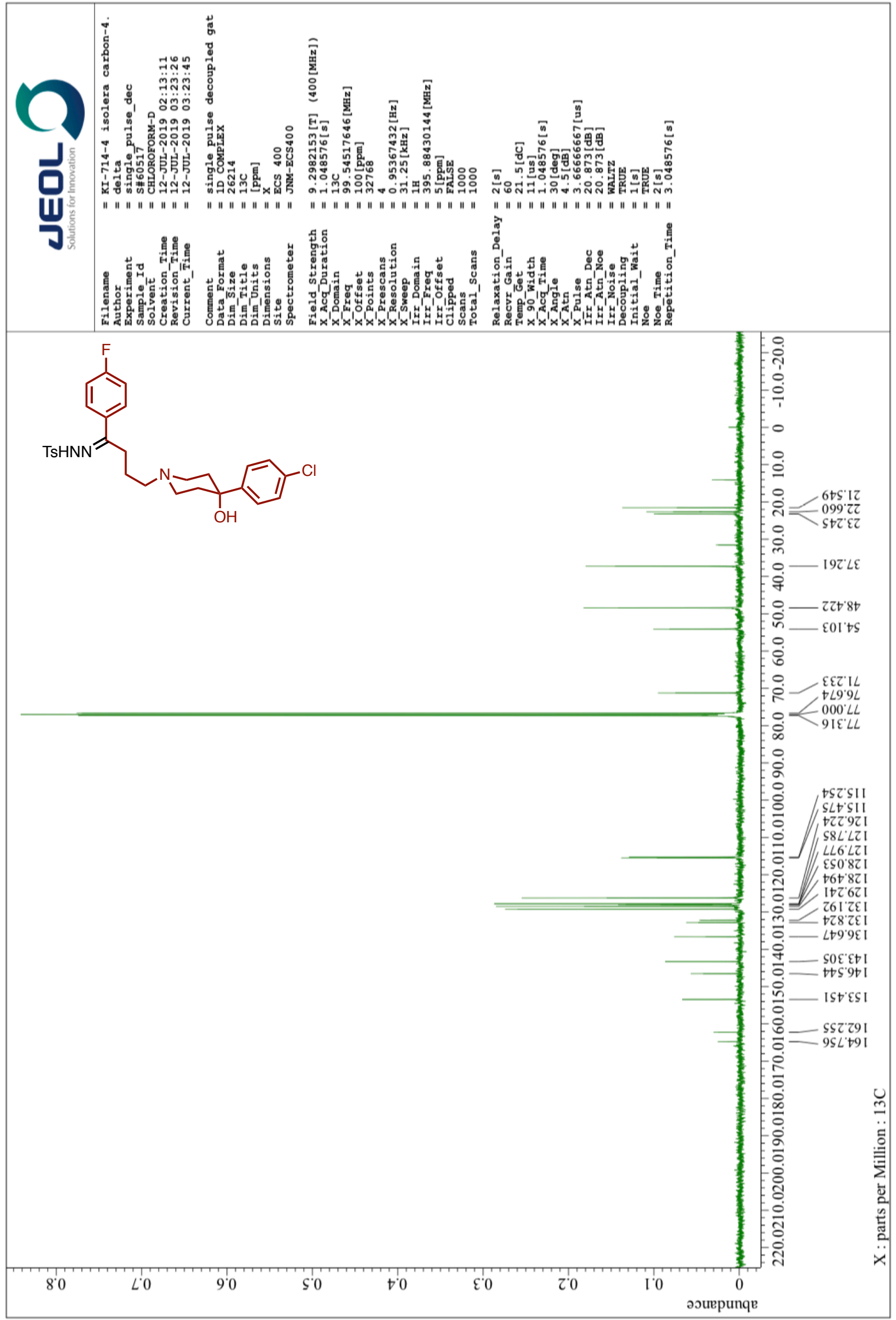


${ }^{1} \mathrm{H}$ NMR of $\mathbf{3 A a}\left(400 \mathrm{MHz}, \mathrm{CDCl}_{3}\right)$

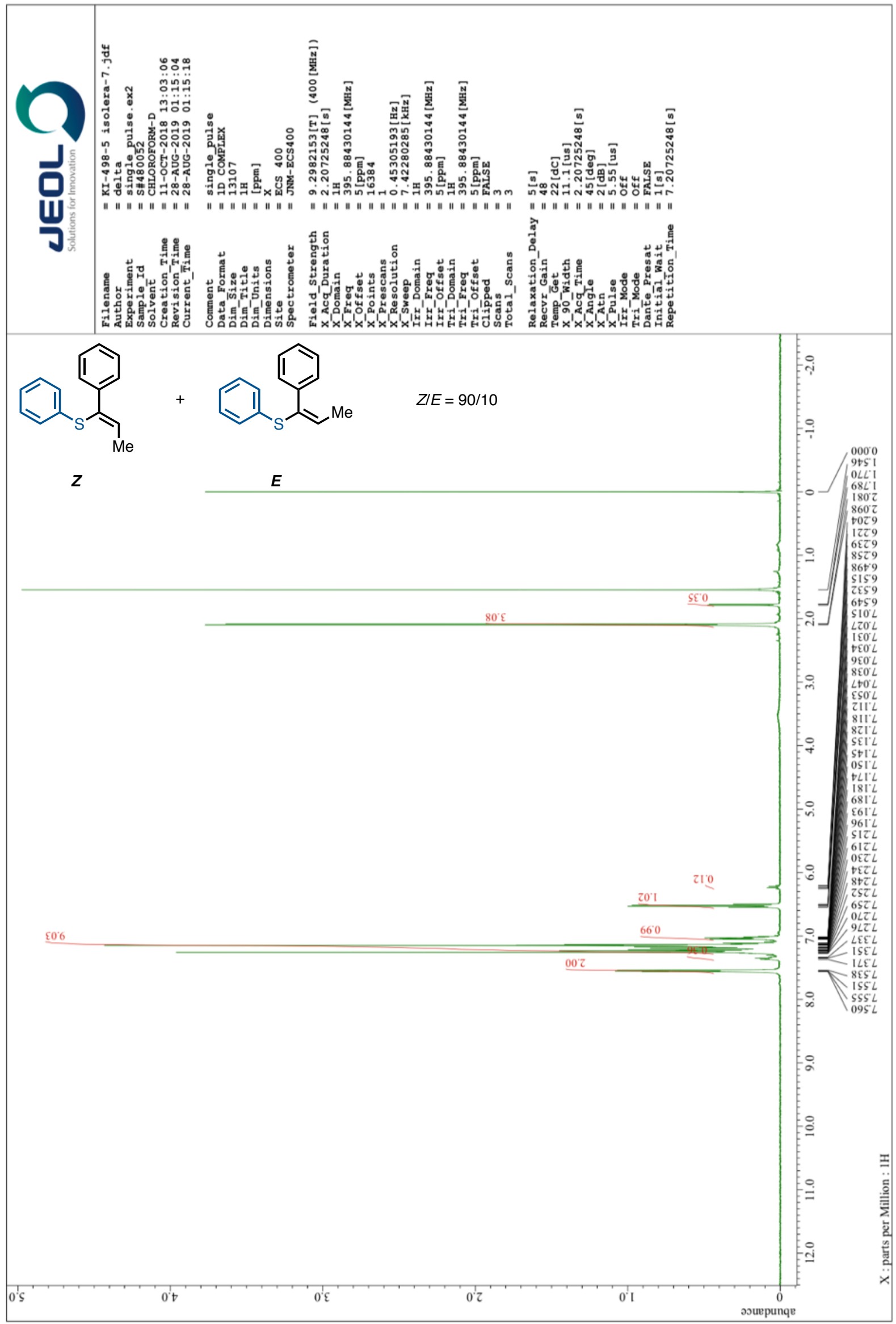


${ }^{13} \mathrm{C}$ NMR of $\mathbf{3 A a}\left(101 \mathrm{MHz}, \mathrm{CDCl}_{3}\right)$

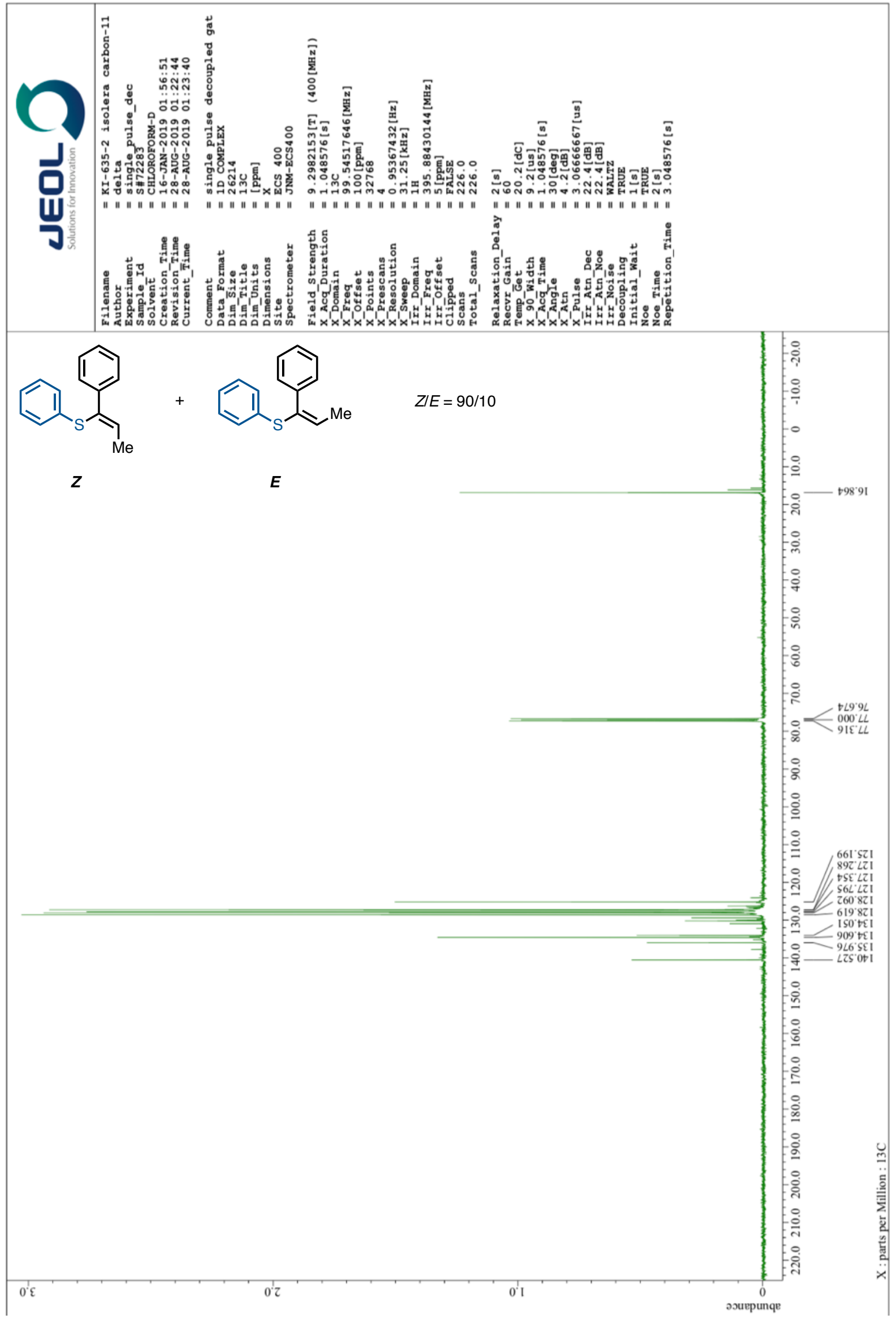


${ }^{1} \mathrm{H}$ NMR of $3 \mathrm{Ba}\left(400 \mathrm{MHz}, \mathrm{CDCl}_{3}\right)$

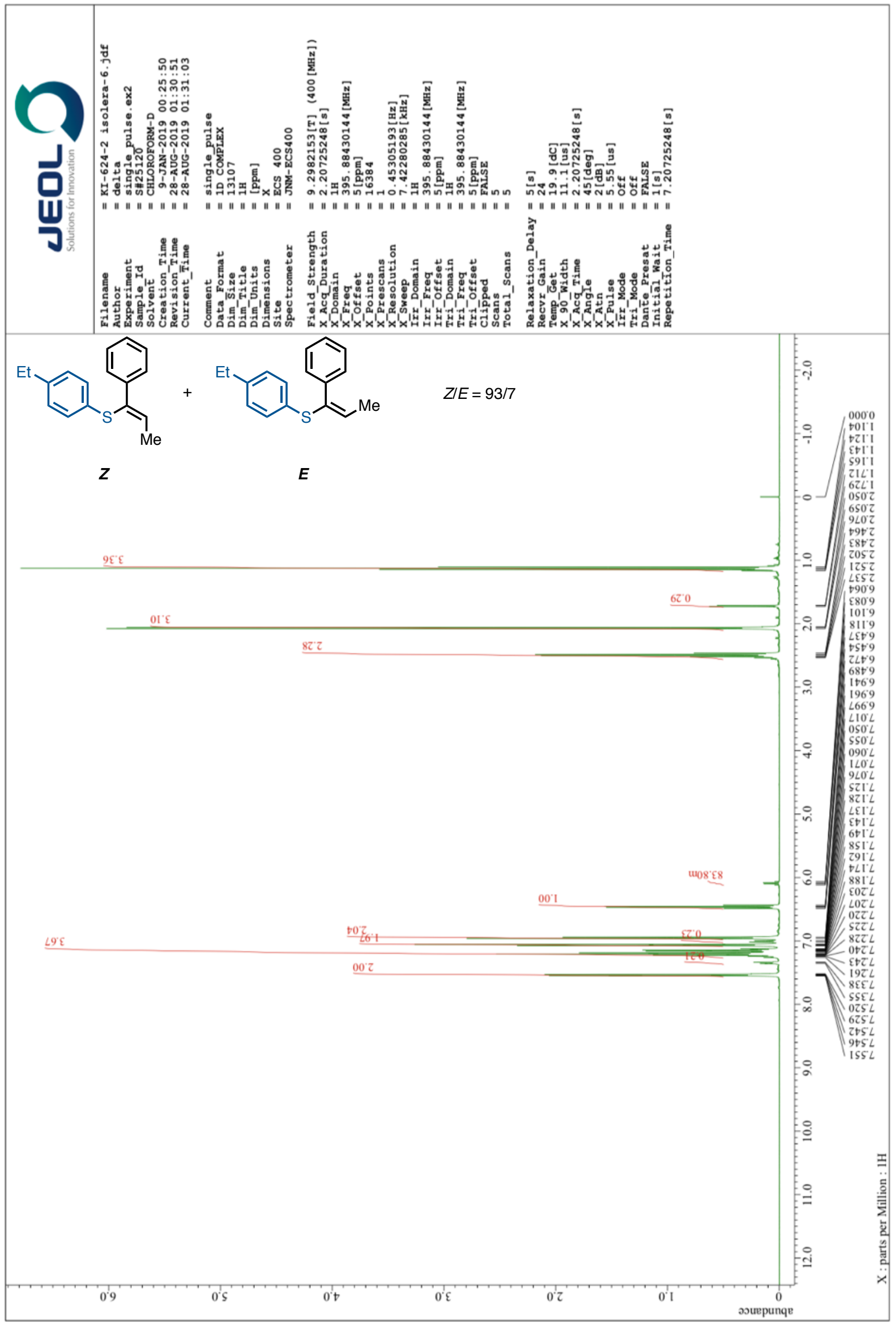


${ }^{13} \mathrm{C}$ NMR of $\mathbf{3 B a}\left(101 \mathrm{MHz}, \mathrm{CDCl}_{3}\right)$

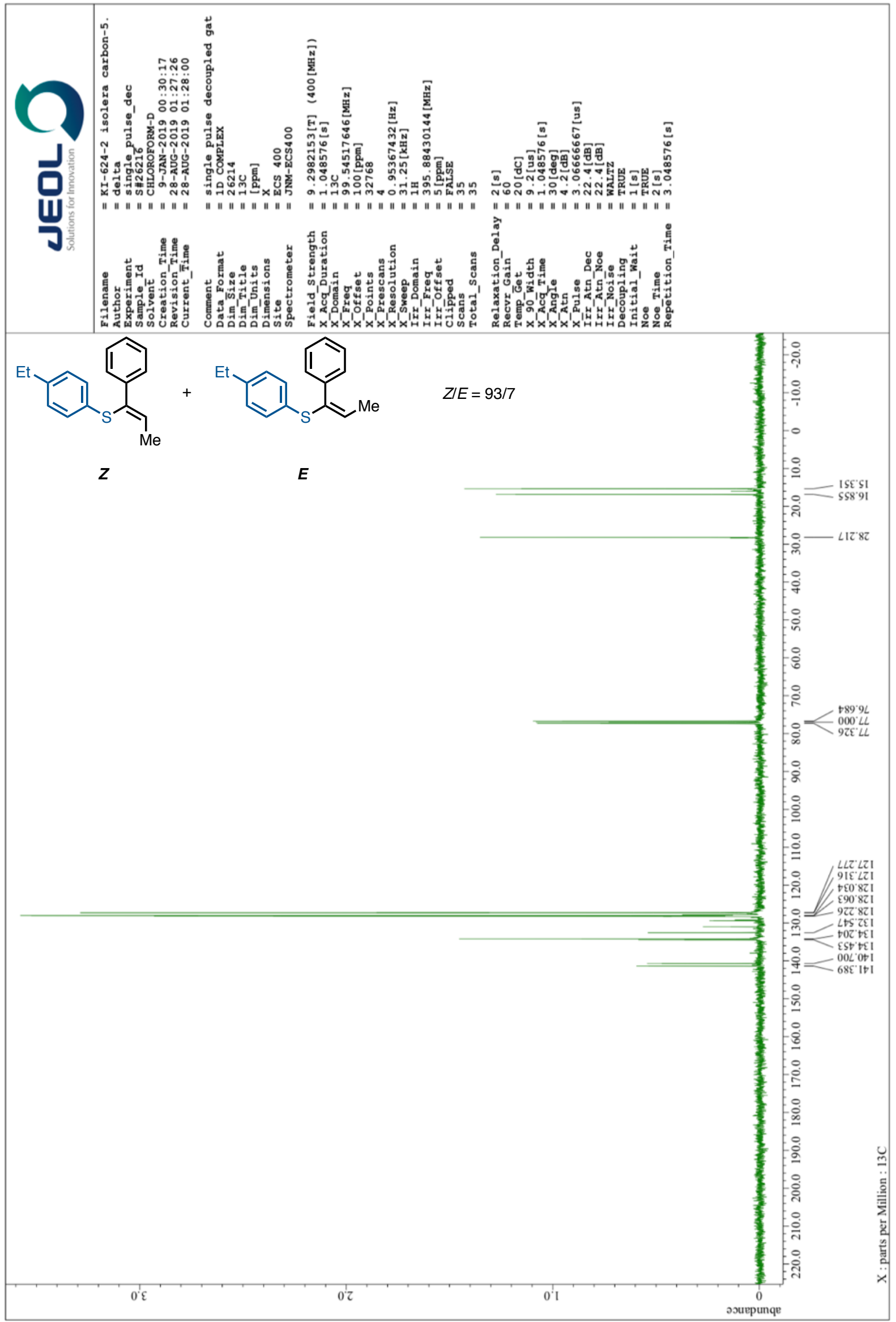


${ }^{1} \mathrm{H}$ NMR of $\mathbf{3 C a}\left(400 \mathrm{MHz}, \mathrm{CDCl}_{3}\right)$

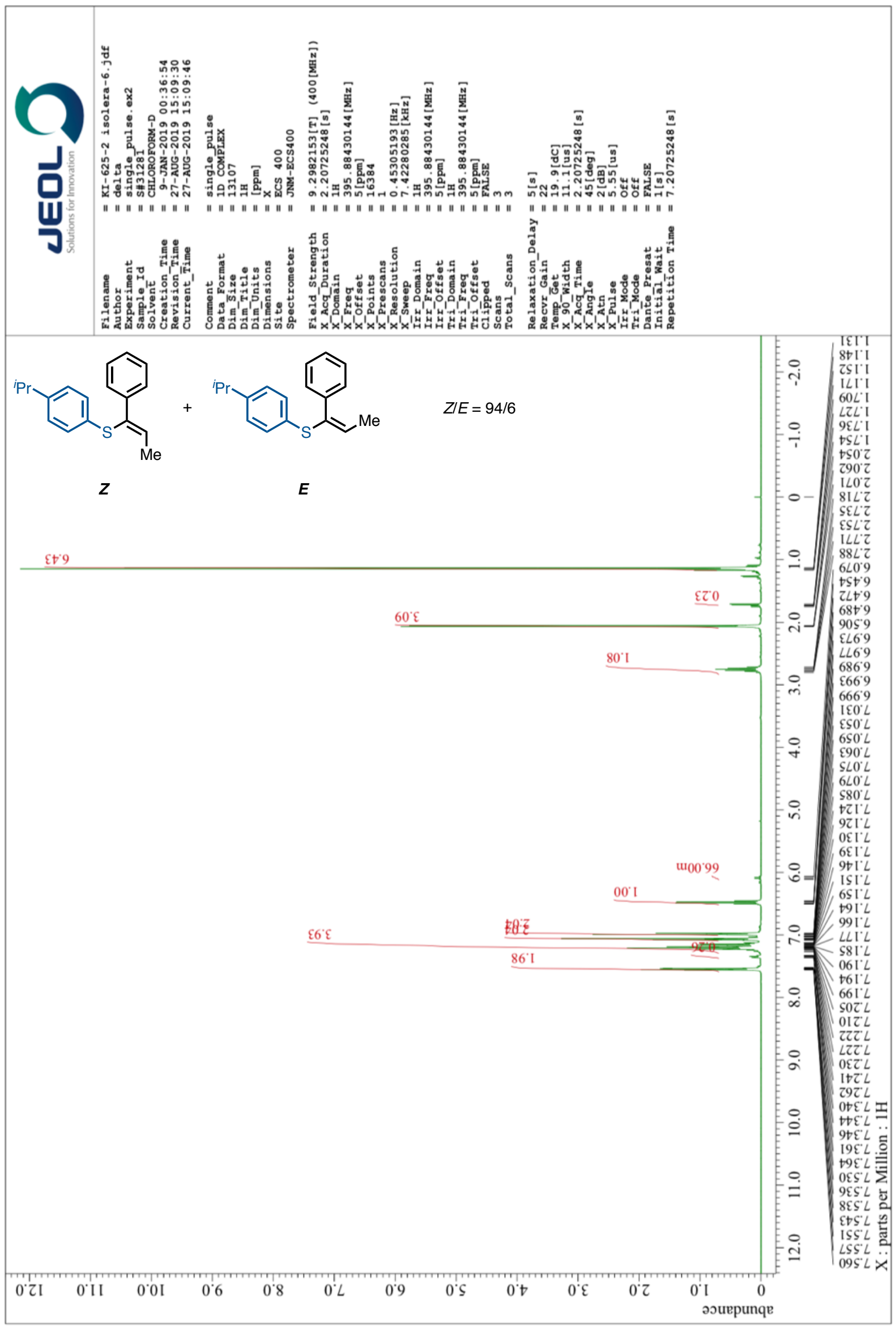


${ }^{13} \mathrm{C}$ NMR of $\mathbf{3 C a}\left(101 \mathrm{MHz}, \mathrm{CDCl}_{3}\right)$

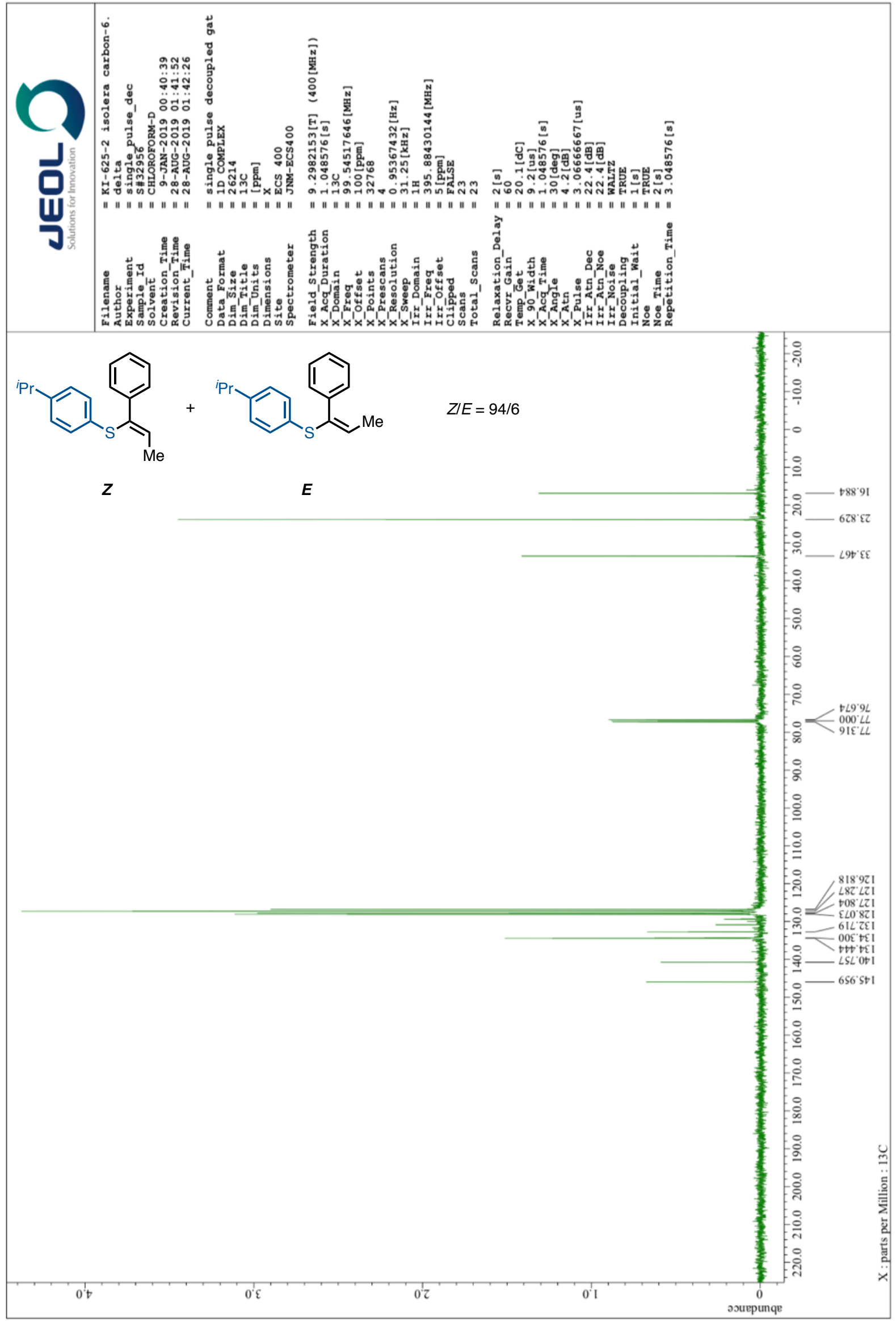


${ }^{1} \mathrm{H}$ NMR of 3Da $\left(400 \mathrm{MHz}, \mathrm{CDCl}_{3}\right)$

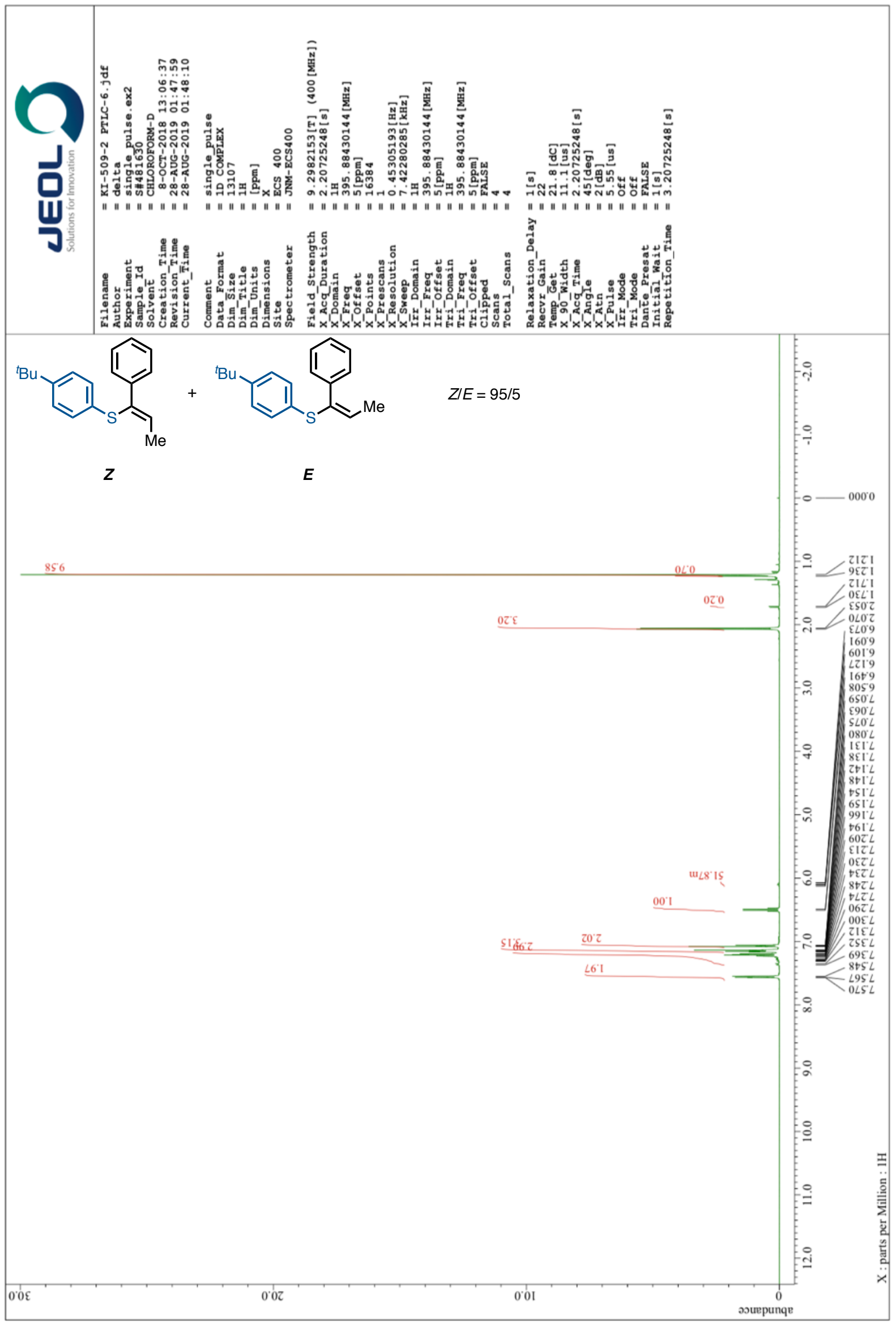


${ }^{13} \mathrm{C}$ NMR of 3Da $\left(101 \mathrm{MHz}, \mathrm{CDCl}_{3}\right)$

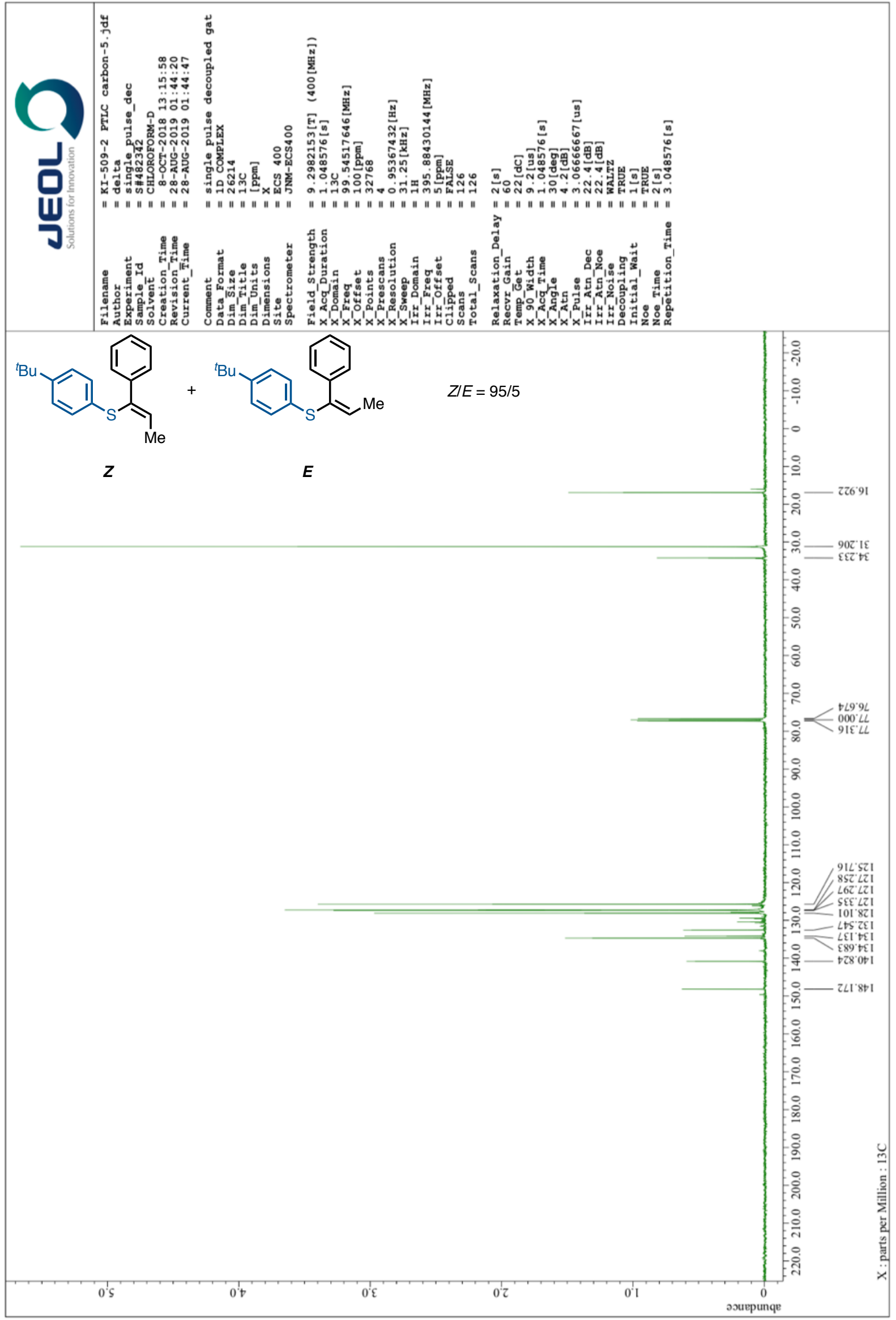


${ }^{1} \mathrm{H}$ NMR of 3Ea $\left(400 \mathrm{MHz}, \mathrm{CDCl}_{3}\right)$

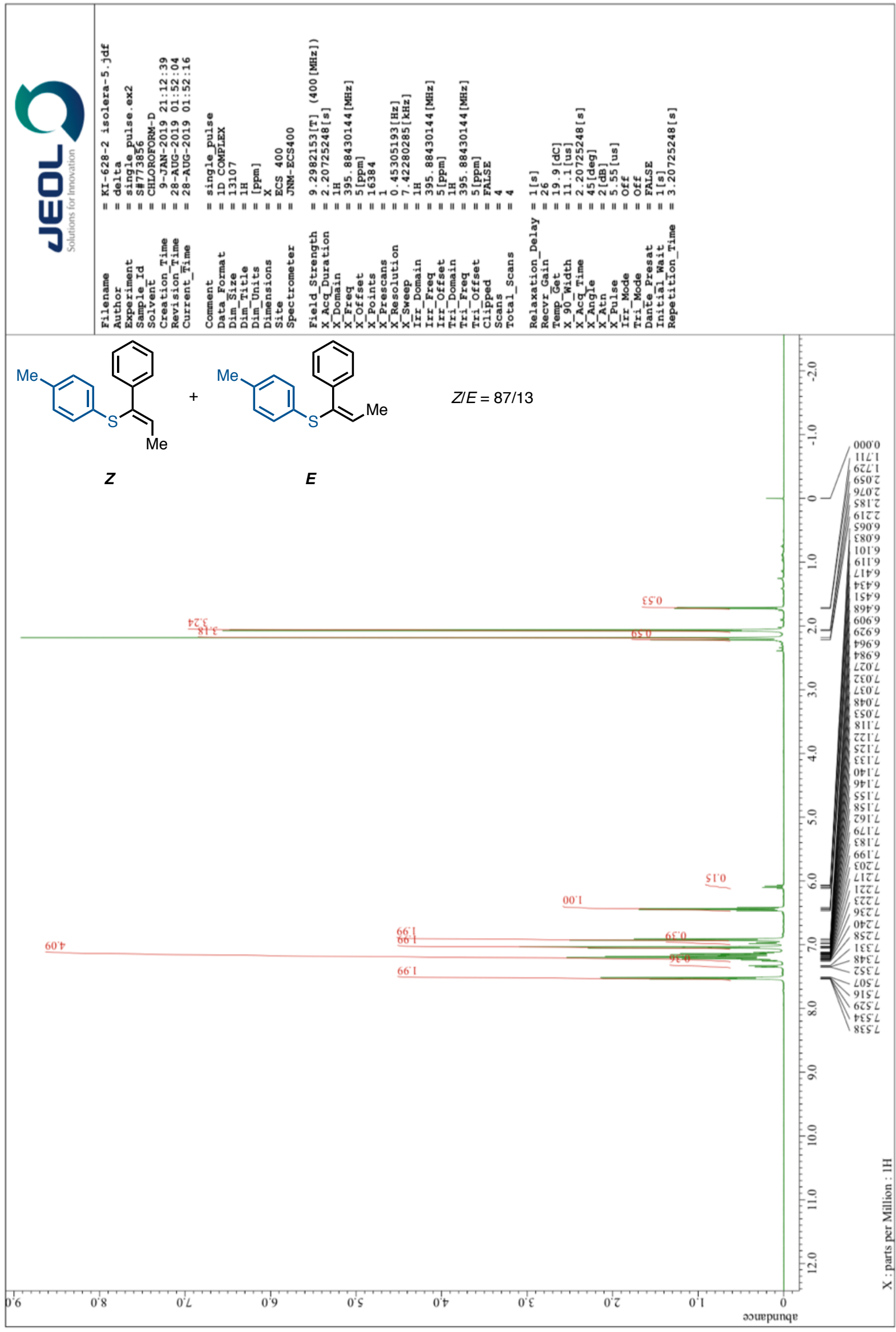


${ }^{13} \mathrm{C}$ NMR of 3Ea $\left(101 \mathrm{MHz}, \mathrm{CDCl}_{3}\right)$

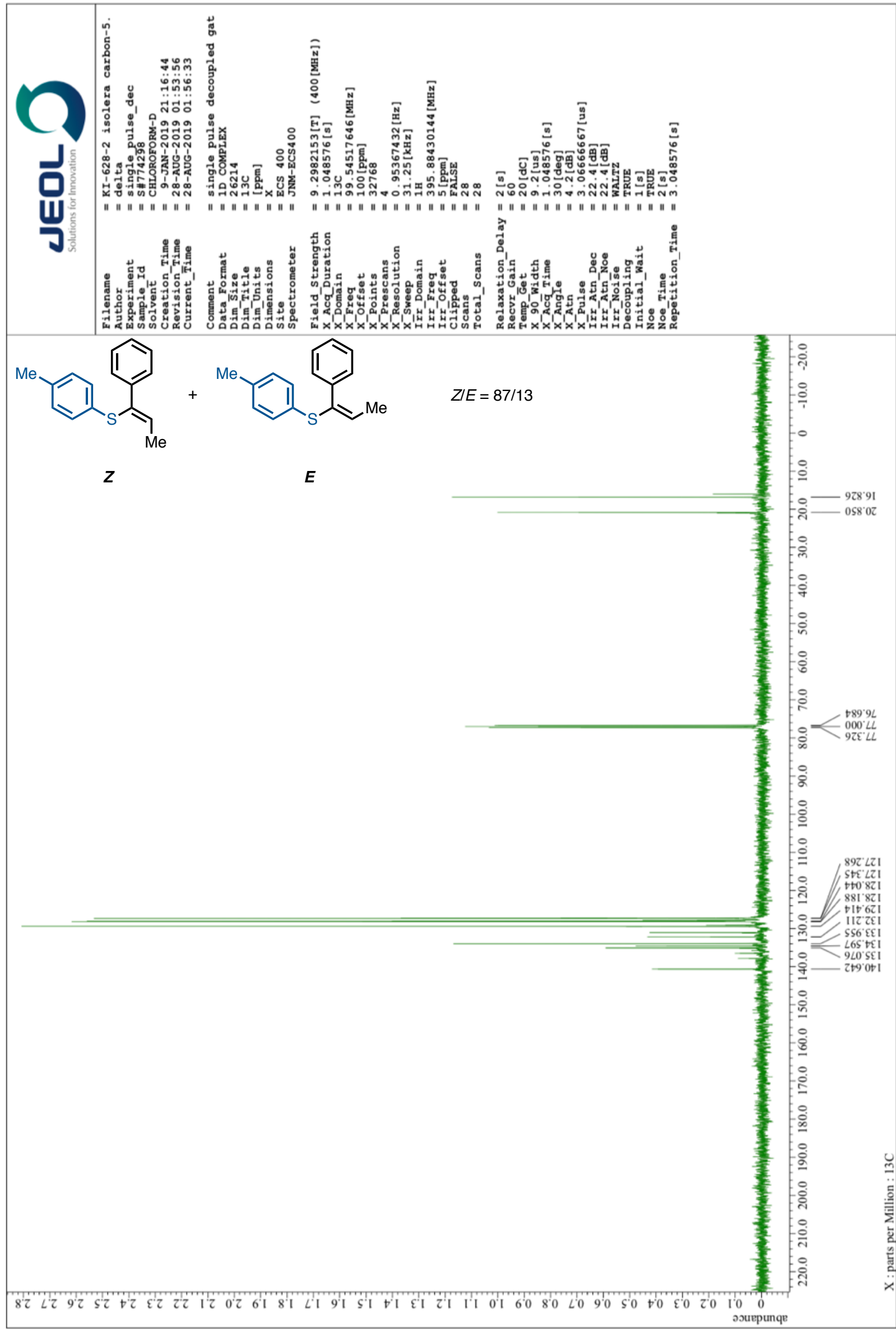


${ }^{1} \mathrm{H}$ NMR of $\mathbf{3 F a}\left(400 \mathrm{MHz}, \mathrm{CDCl}_{3}\right)$

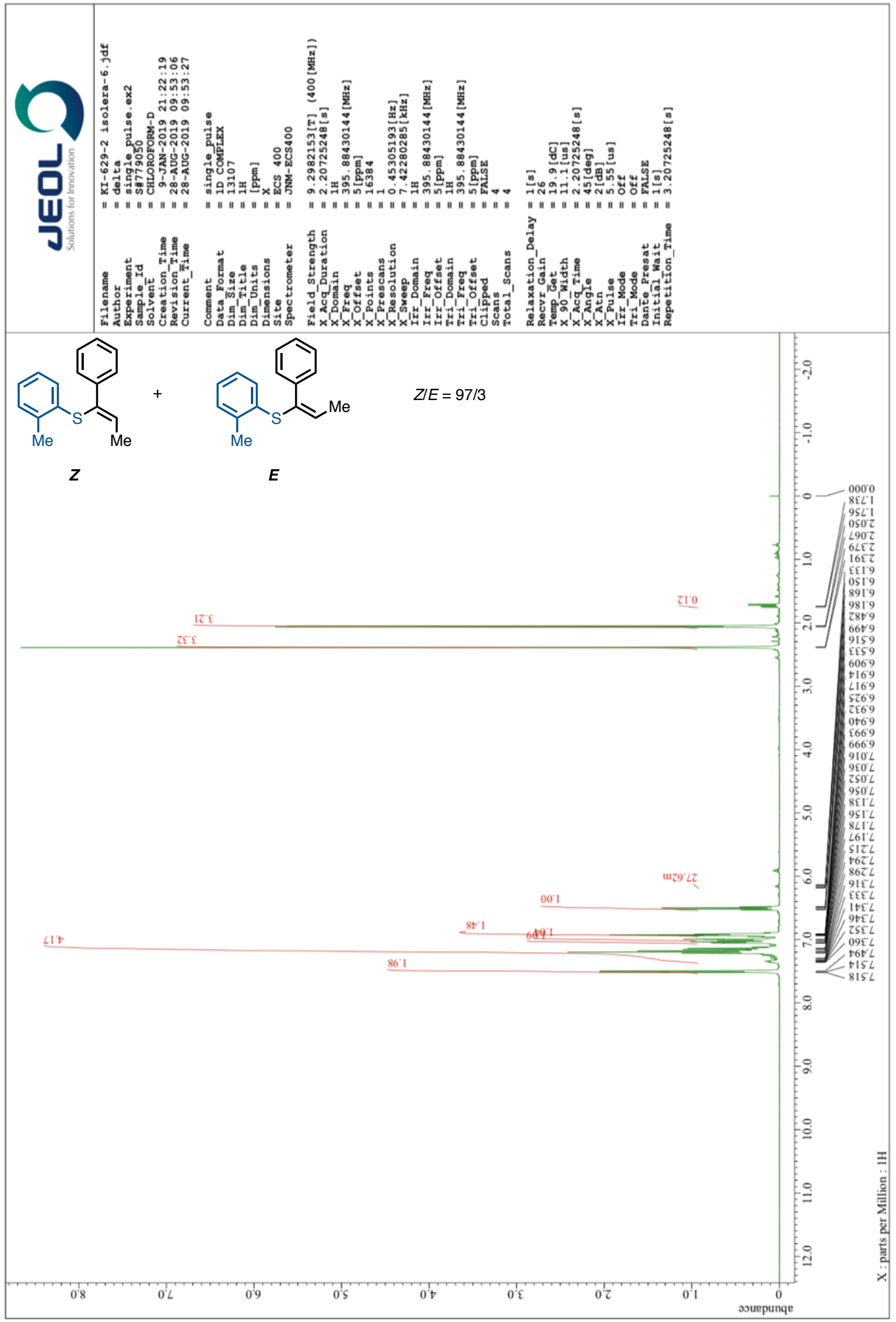


${ }^{13} \mathrm{C}$ NMR of $\mathbf{3 F a}\left(101 \mathrm{MHz}, \mathrm{CDCl}_{3}\right)$

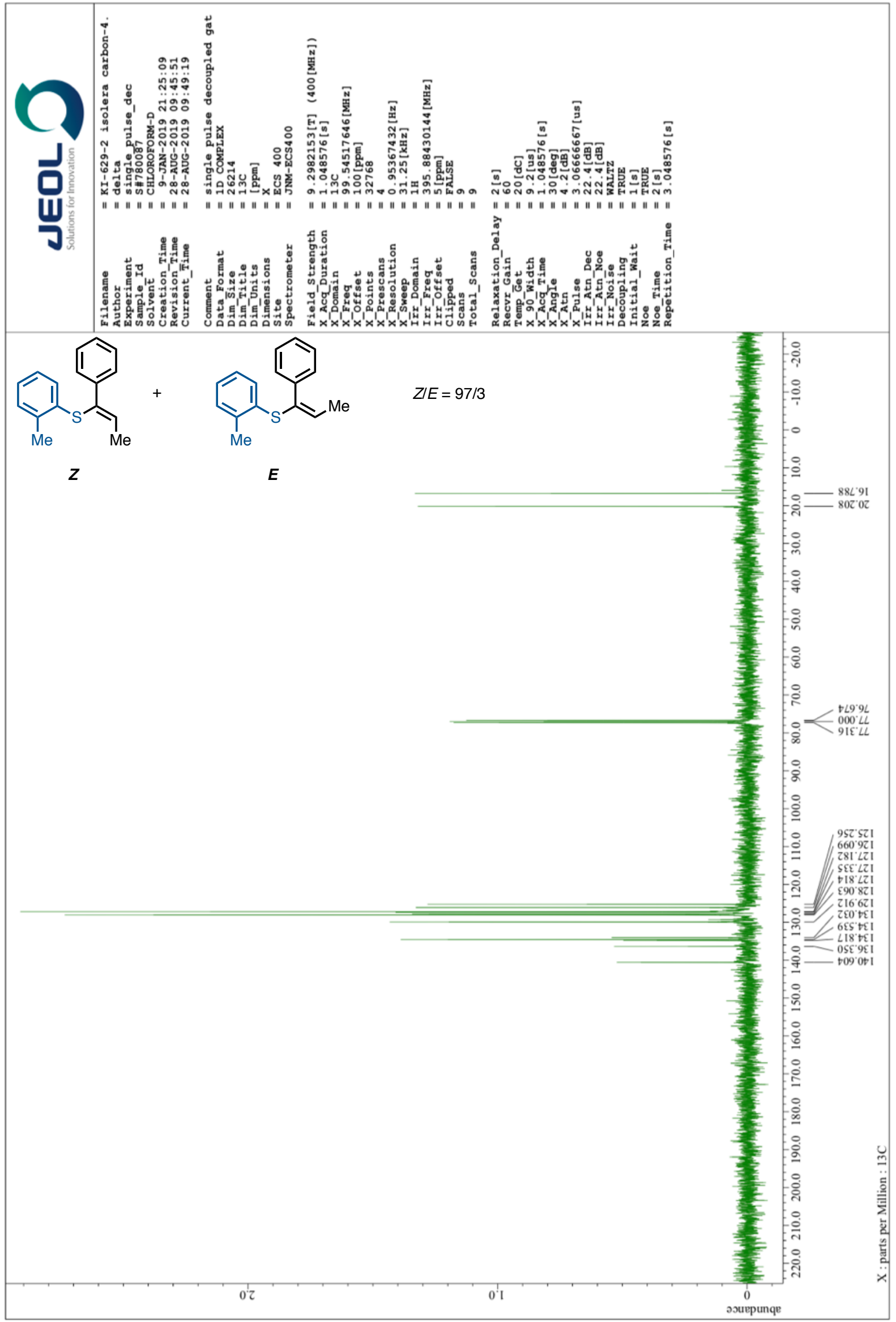


${ }^{1} \mathrm{H}$ NMR of $\mathbf{3 G a}\left(400 \mathrm{MHz}, \mathrm{CDCl}_{3}\right)$

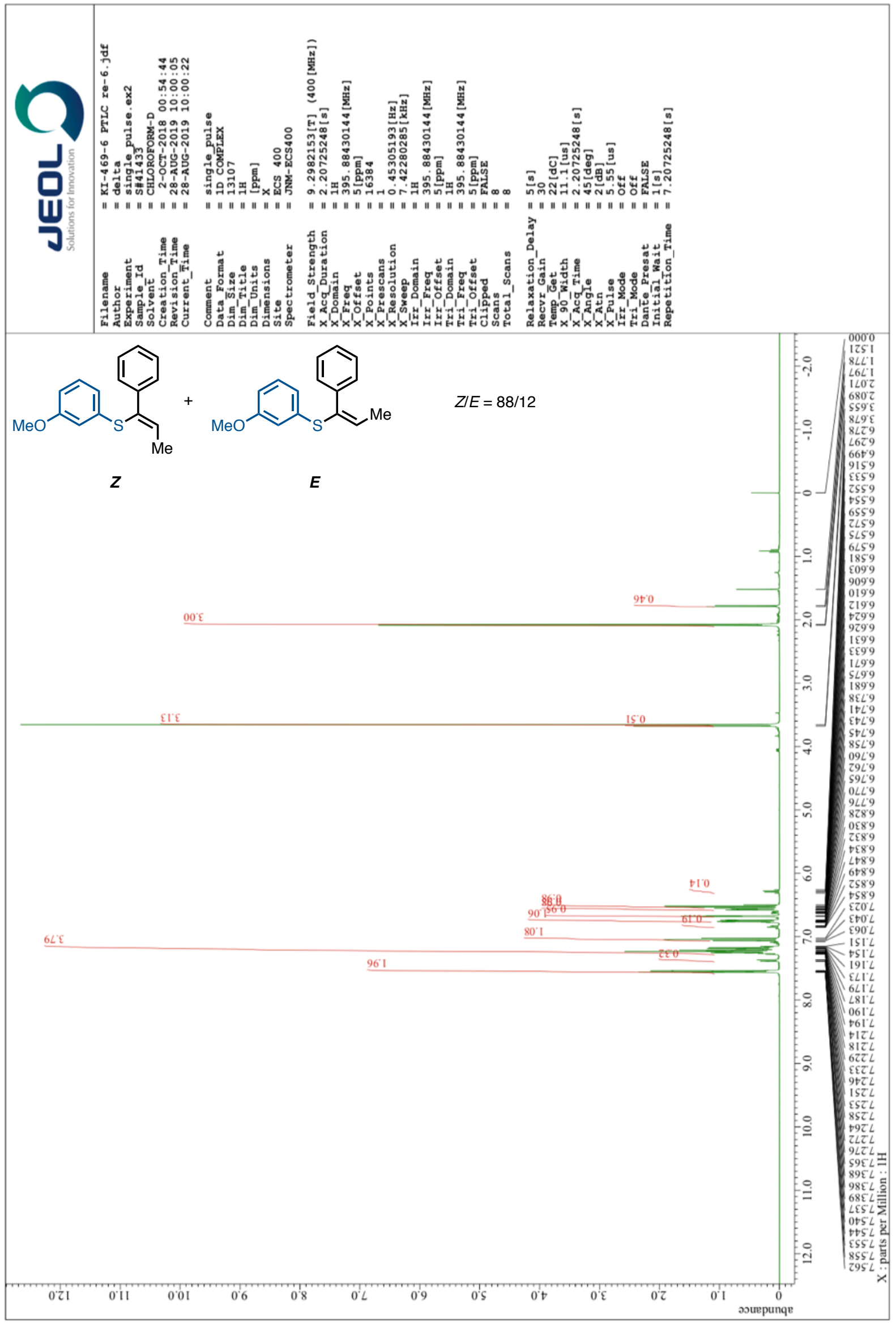


${ }^{13} \mathrm{C}$ NMR of $\mathbf{3 G a}\left(101 \mathrm{MHz}, \mathrm{CDCl}_{3}\right)$

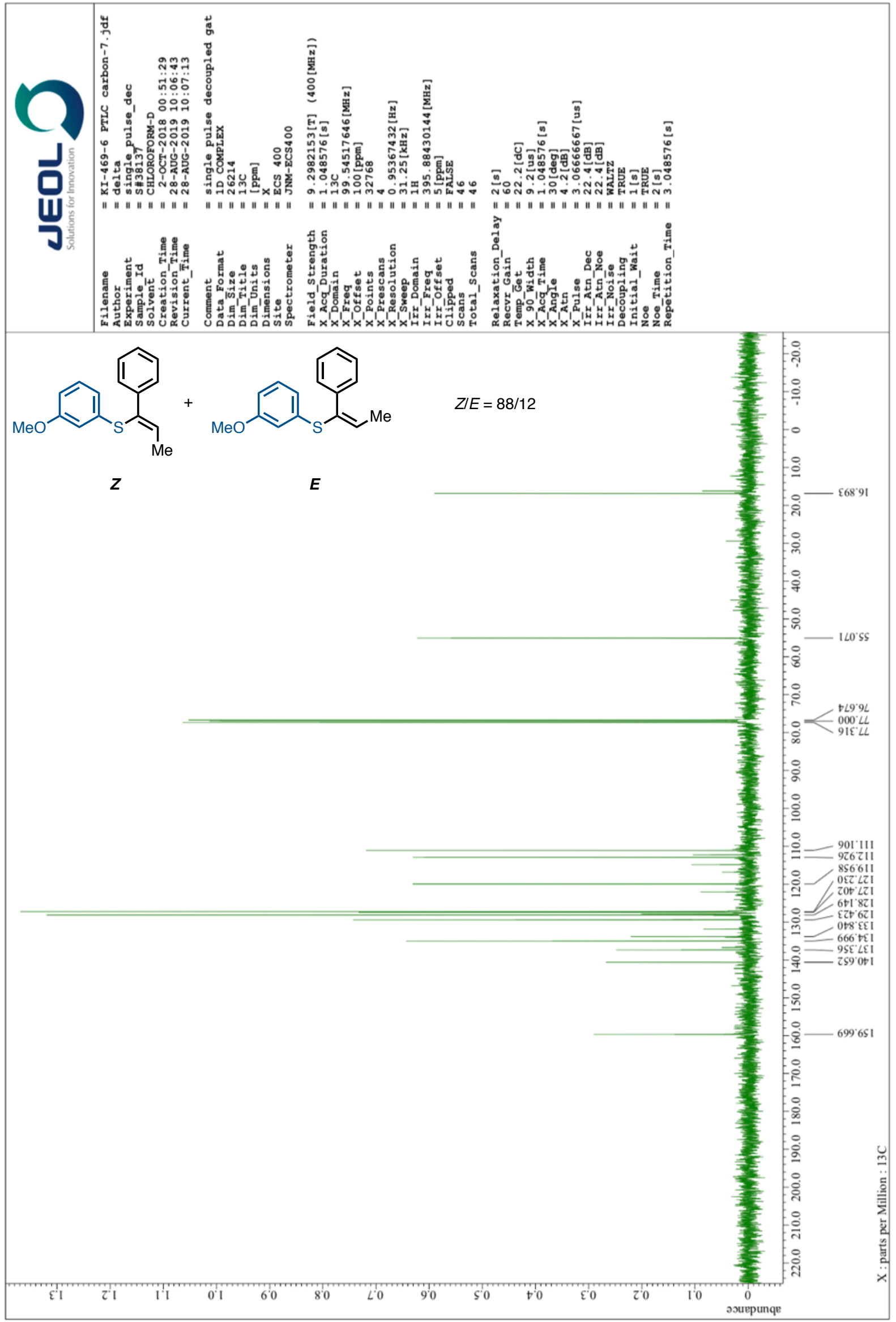


${ }^{1} \mathrm{H}$ NMR of $\mathbf{3 H a}\left(400 \mathrm{MHz}, \mathrm{CDCl}_{3}\right)$

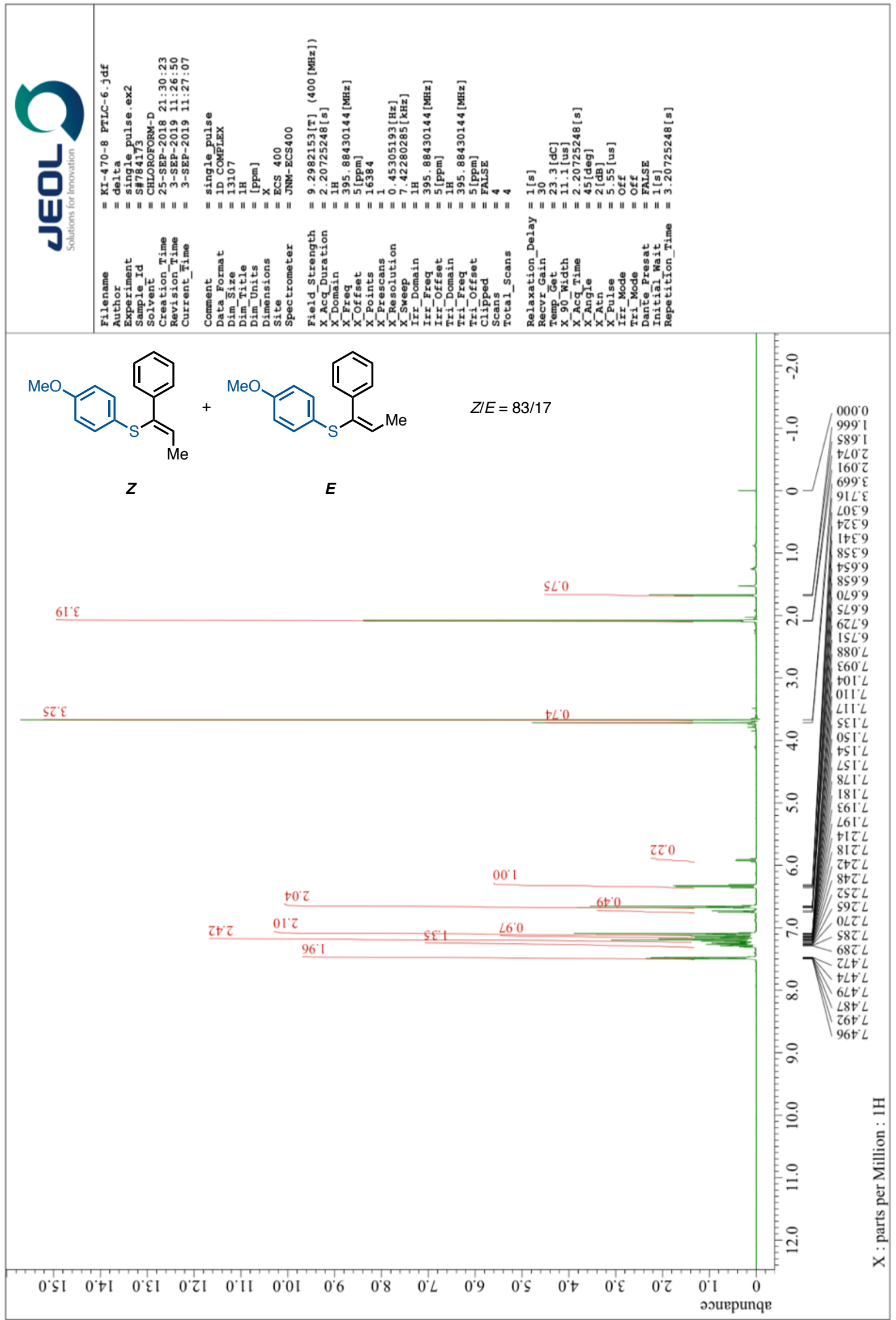


${ }^{13} \mathrm{C}$ NMR of $\mathbf{3 H a}\left(101 \mathrm{MHz}, \mathrm{CDCl}_{3}\right)$

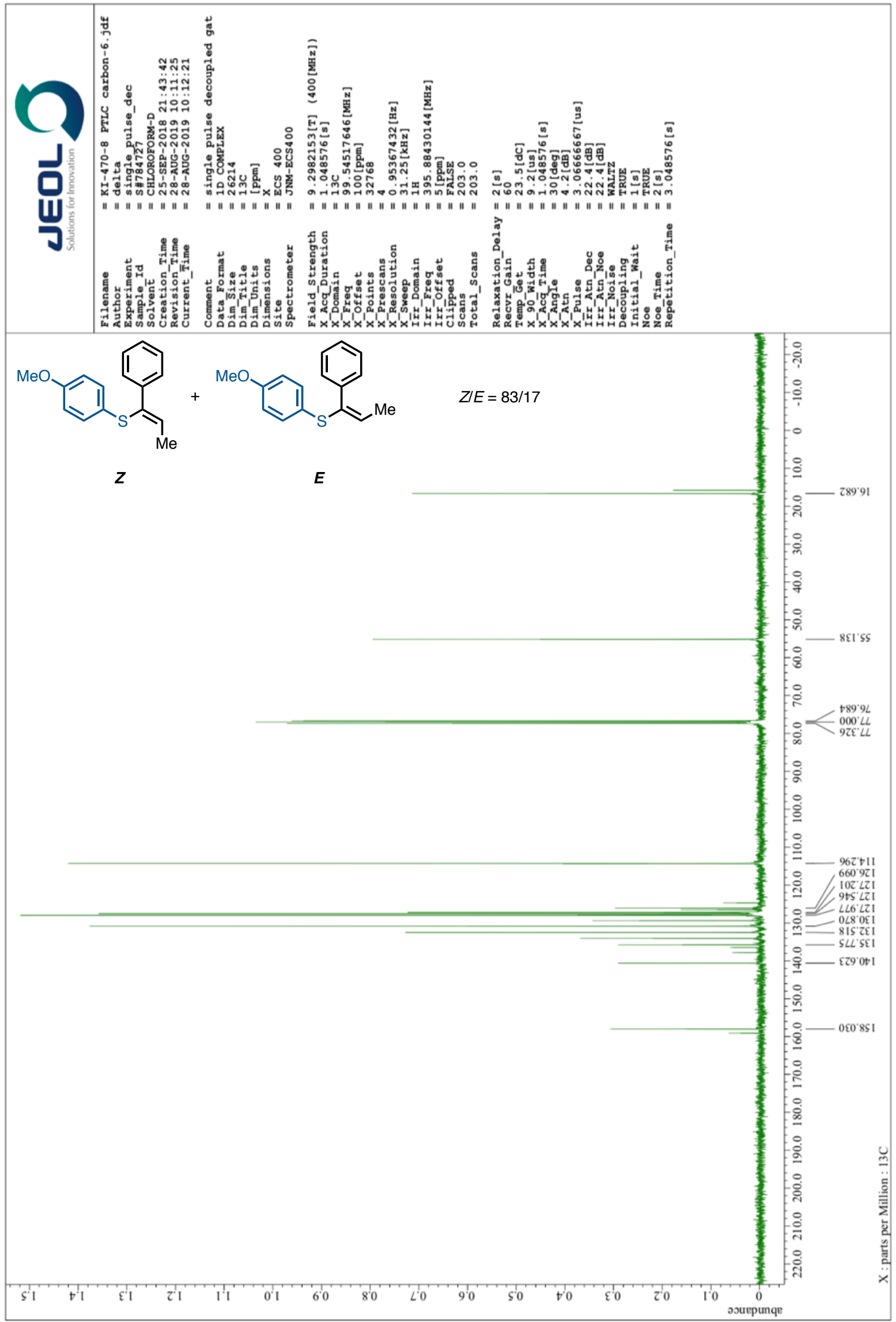


${ }^{1} \mathrm{H}$ NMR of $3 \mathbf{I a}\left(400 \mathrm{MHz}, \mathrm{CDCl}_{3}\right)$

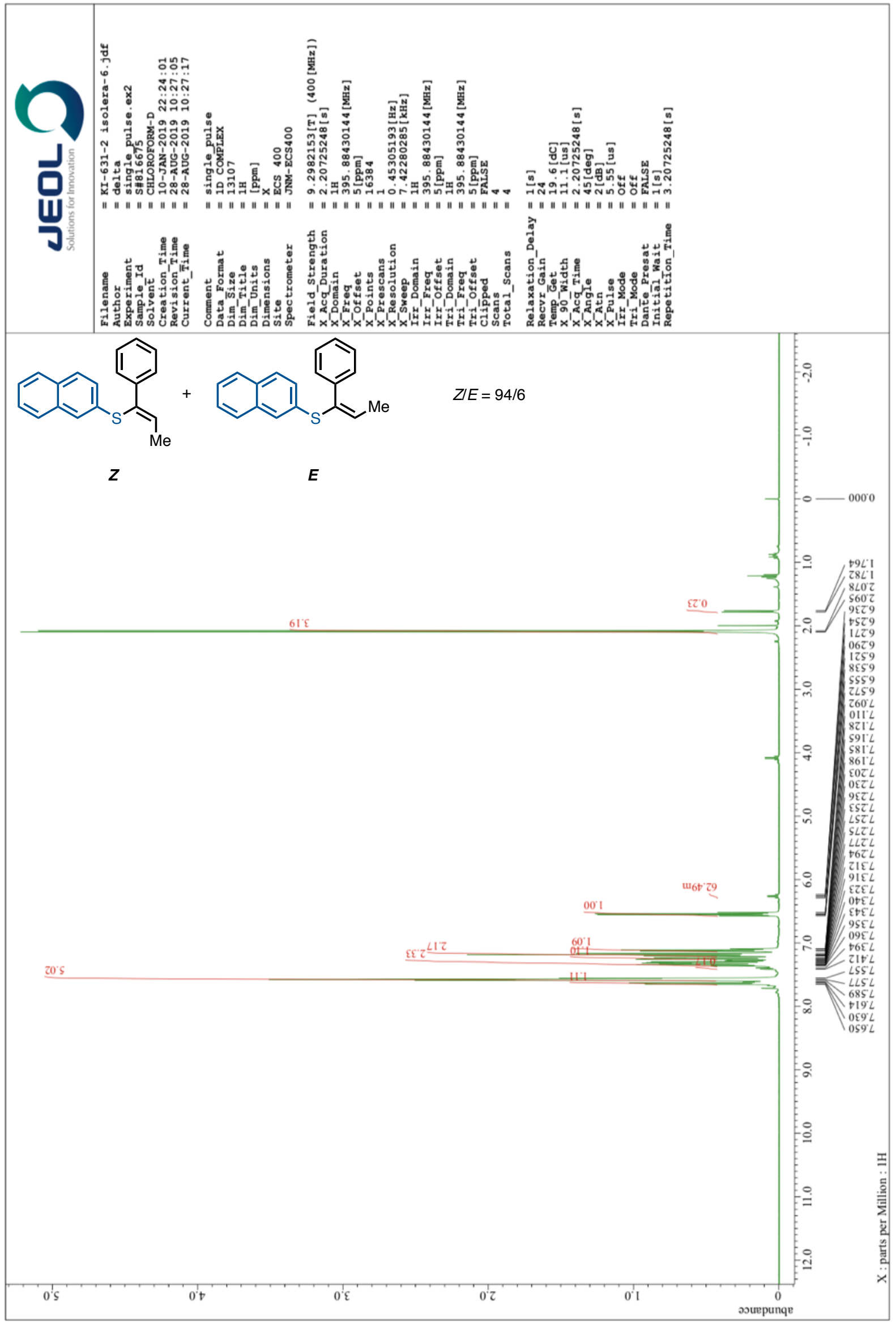


${ }^{13} \mathrm{C}$ NMR of 3Ia $\left(101 \mathrm{MHz}, \mathrm{CDCl}_{3}\right)$

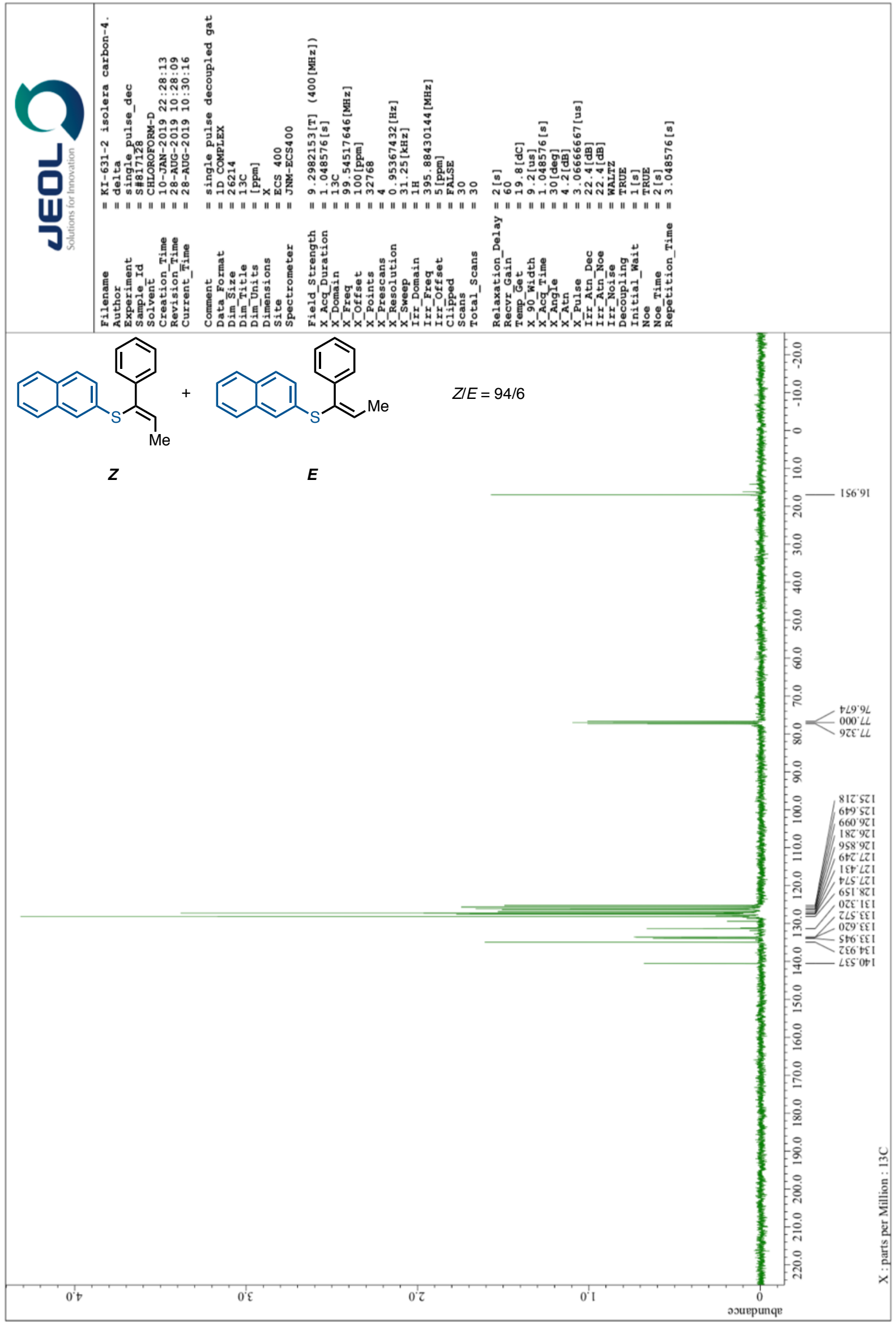


${ }^{1} \mathrm{H}$ NMR of $\mathbf{3 J a}\left(400 \mathrm{MHz}, \mathrm{CDCl}_{3}\right)$

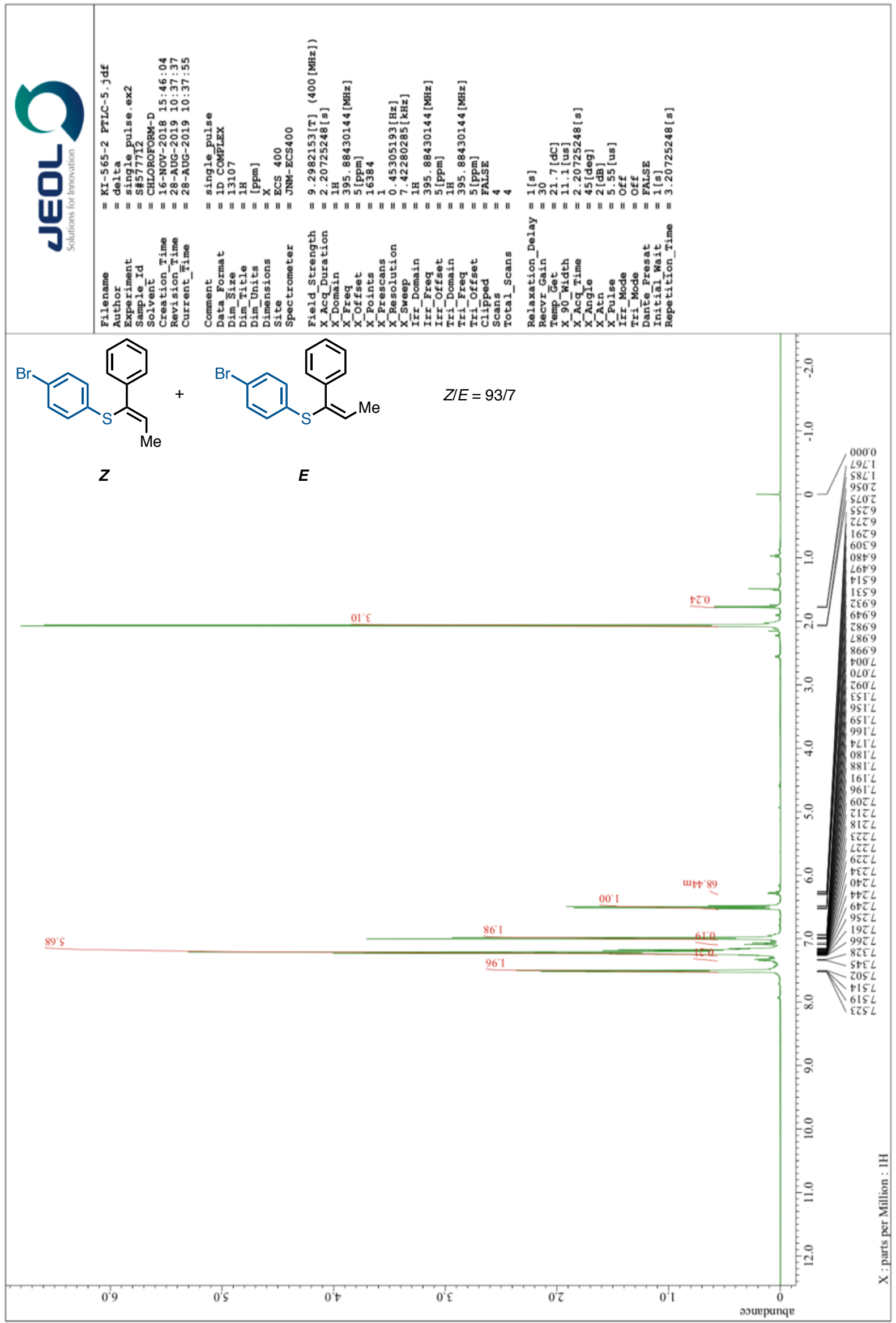


${ }^{13} \mathrm{C}$ NMR of $\mathbf{3 J a}\left(101 \mathrm{MHz}, \mathrm{CDCl}_{3}\right)$

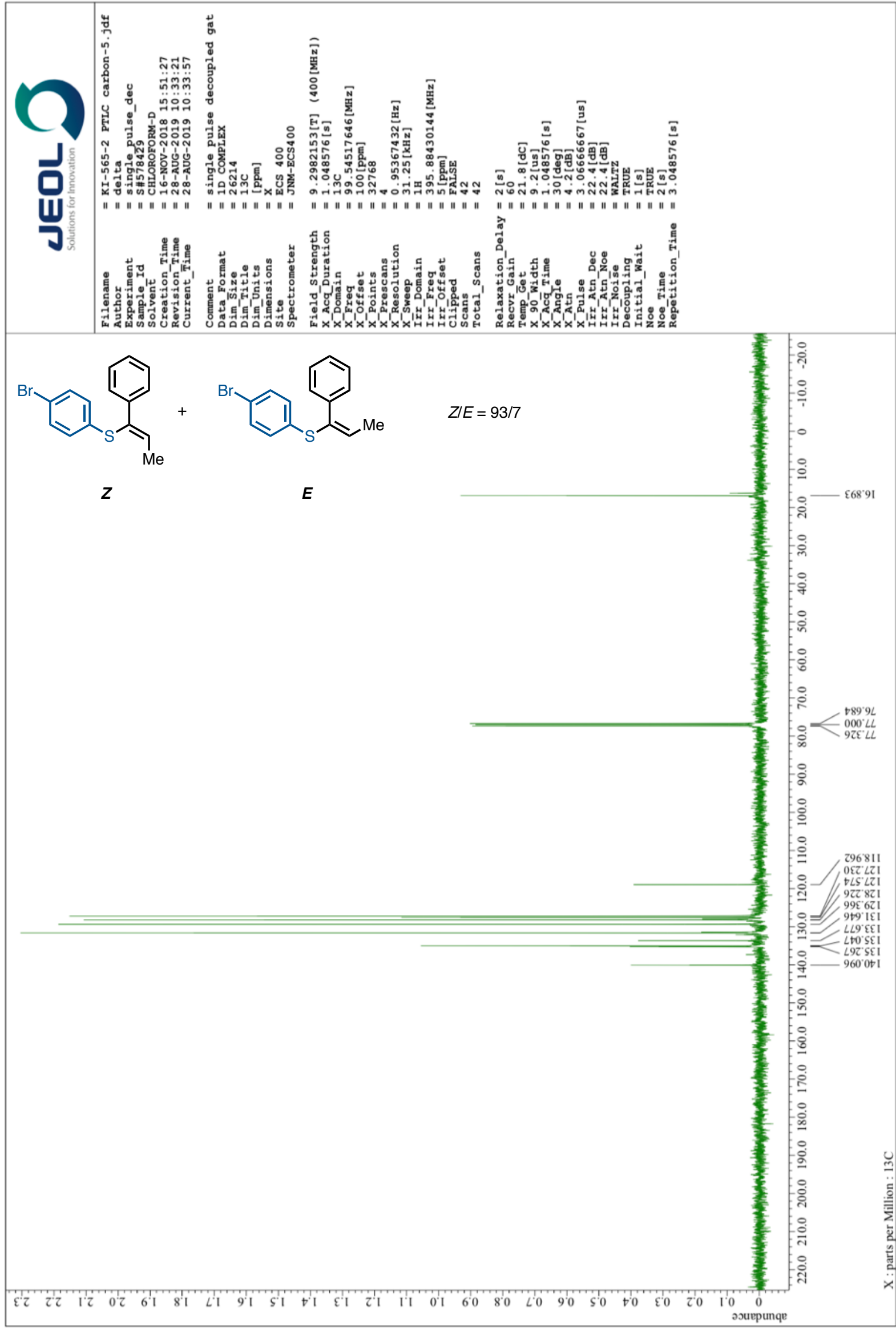


${ }^{1} \mathrm{H}$ NMR of $\mathbf{3 K a}\left(400 \mathrm{MHz}, \mathrm{CDCl}_{3}\right)$

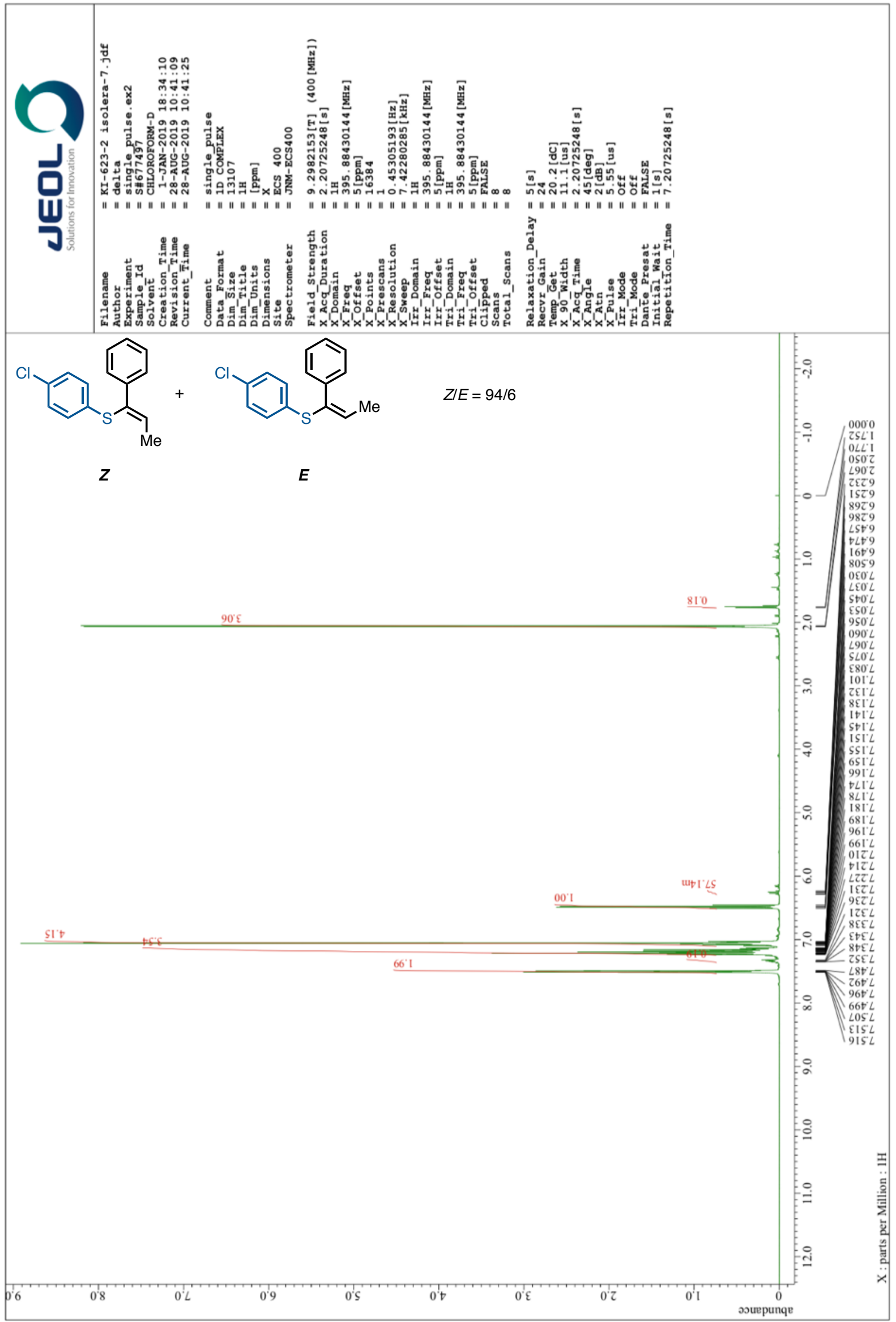


${ }^{13} \mathrm{C}$ NMR of $\mathbf{3 K a}\left(101 \mathrm{MHz}, \mathrm{CDCl}_{3}\right)$

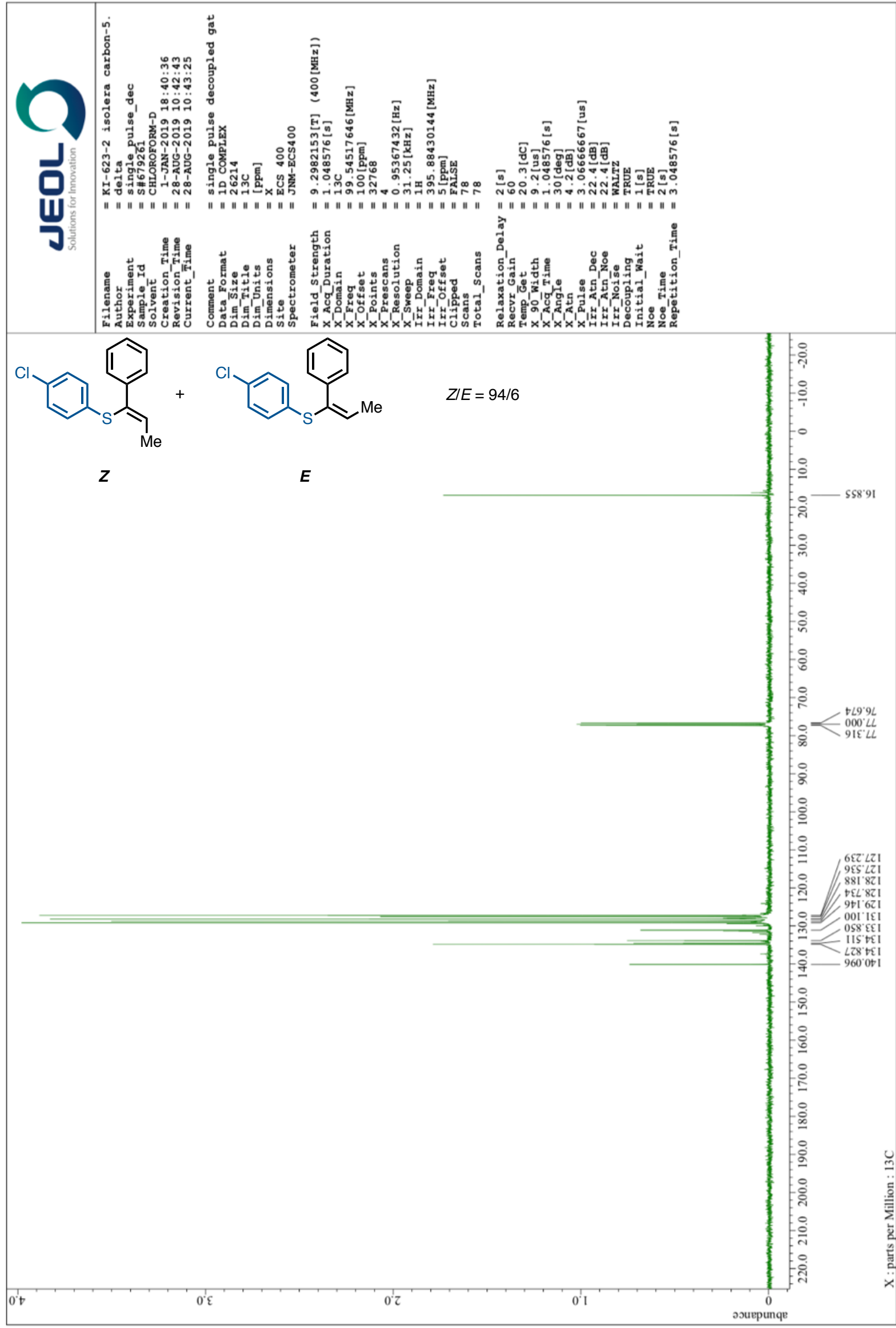


Supporting Information (Ishitobi, Muto, Yamaguchi) Pd-Catalyzed Alkenyl Thioether Synthesis from Thioesters and N-Tosylhydrazones

${ }^{1} \mathrm{H}$ NMR of $\mathbf{3 L a}\left(400 \mathrm{MHz}, \mathrm{CDCl}_{3}\right)$

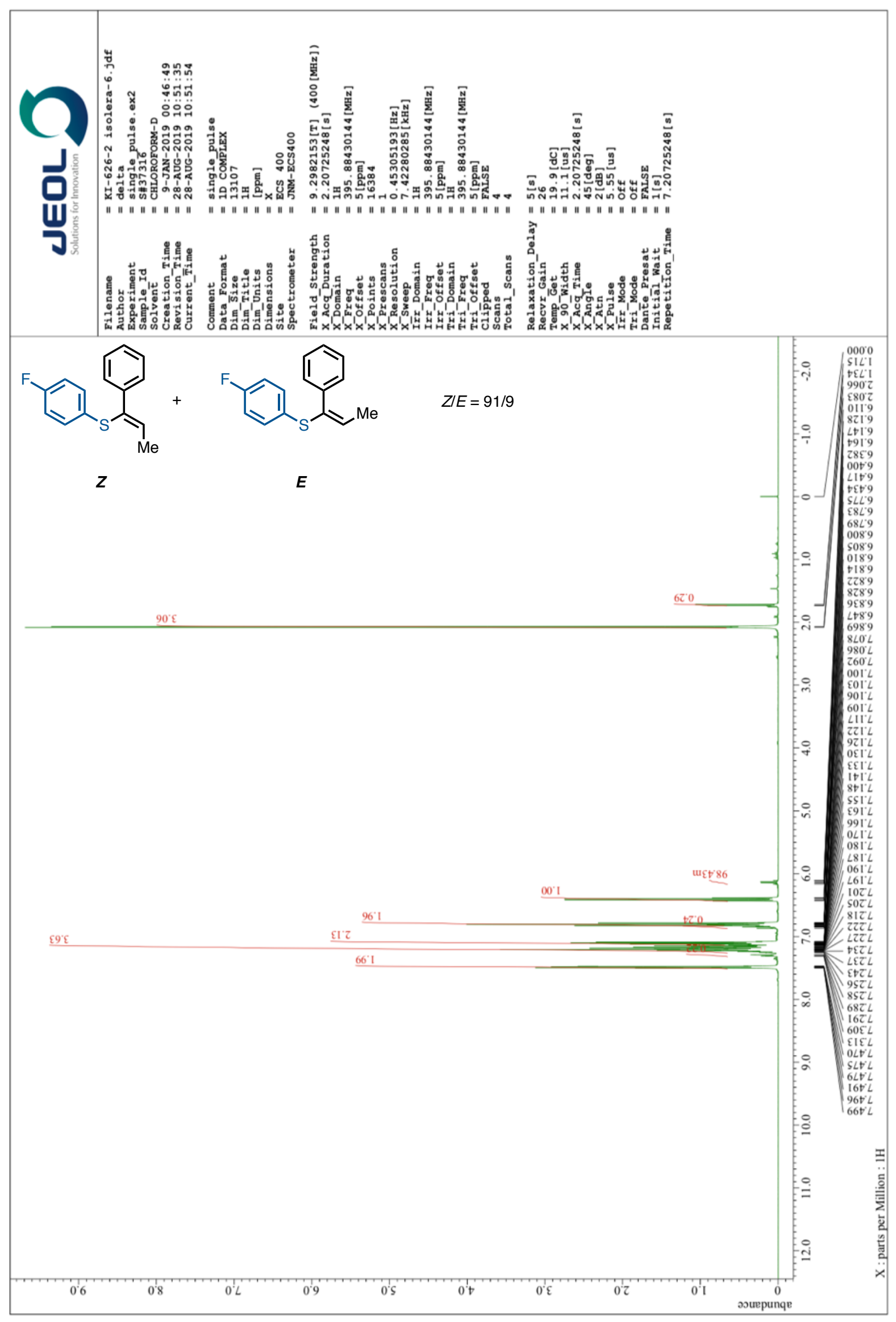

S97 
${ }^{13} \mathrm{C}$ NMR of $\mathbf{3 L a}\left(101 \mathrm{MHz}, \mathrm{CDCl}_{3}\right)$

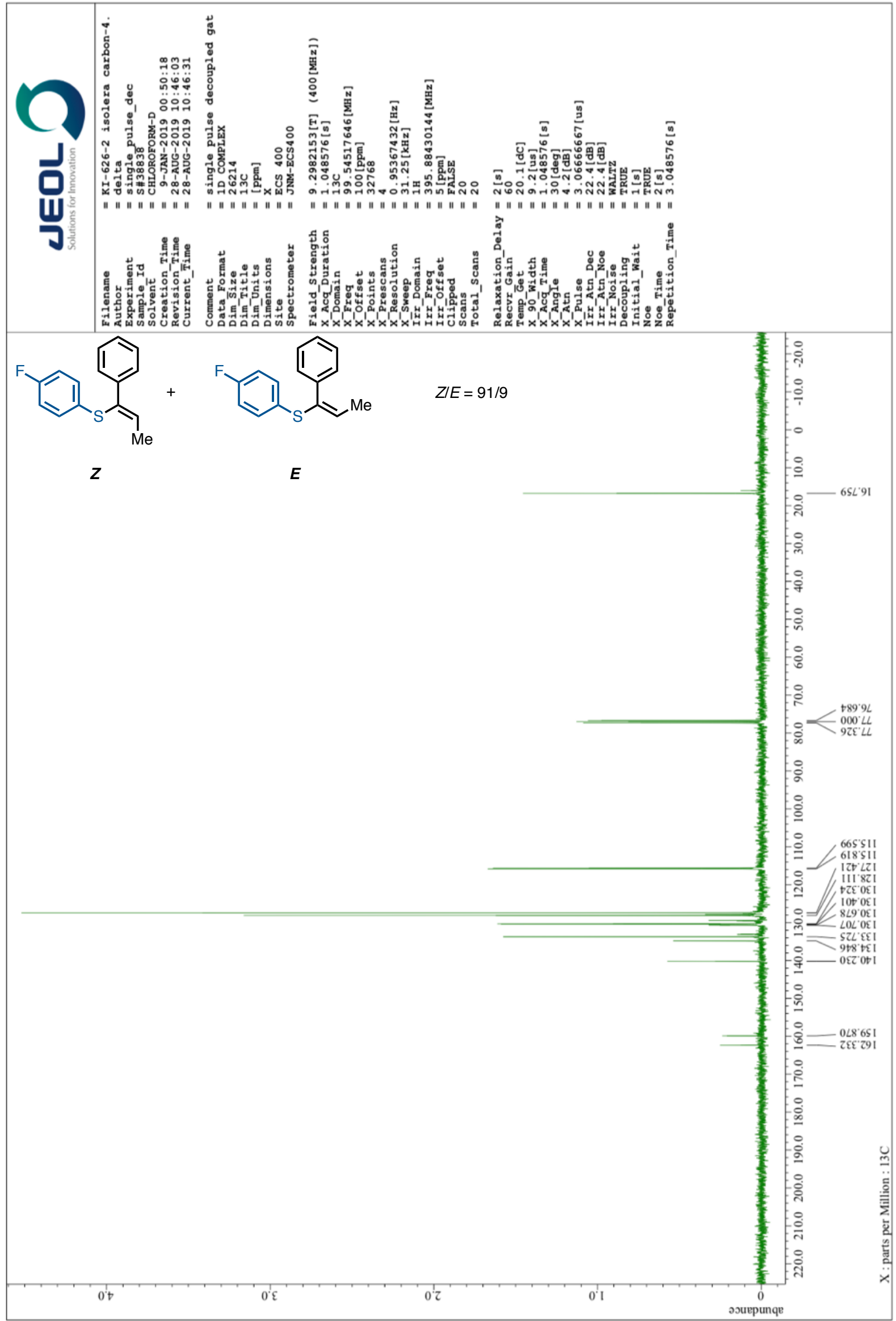


${ }^{1} \mathrm{H}$ NMR of $\mathbf{3 M a}\left(400 \mathrm{MHz}, \mathrm{CDCl}_{3}\right)$

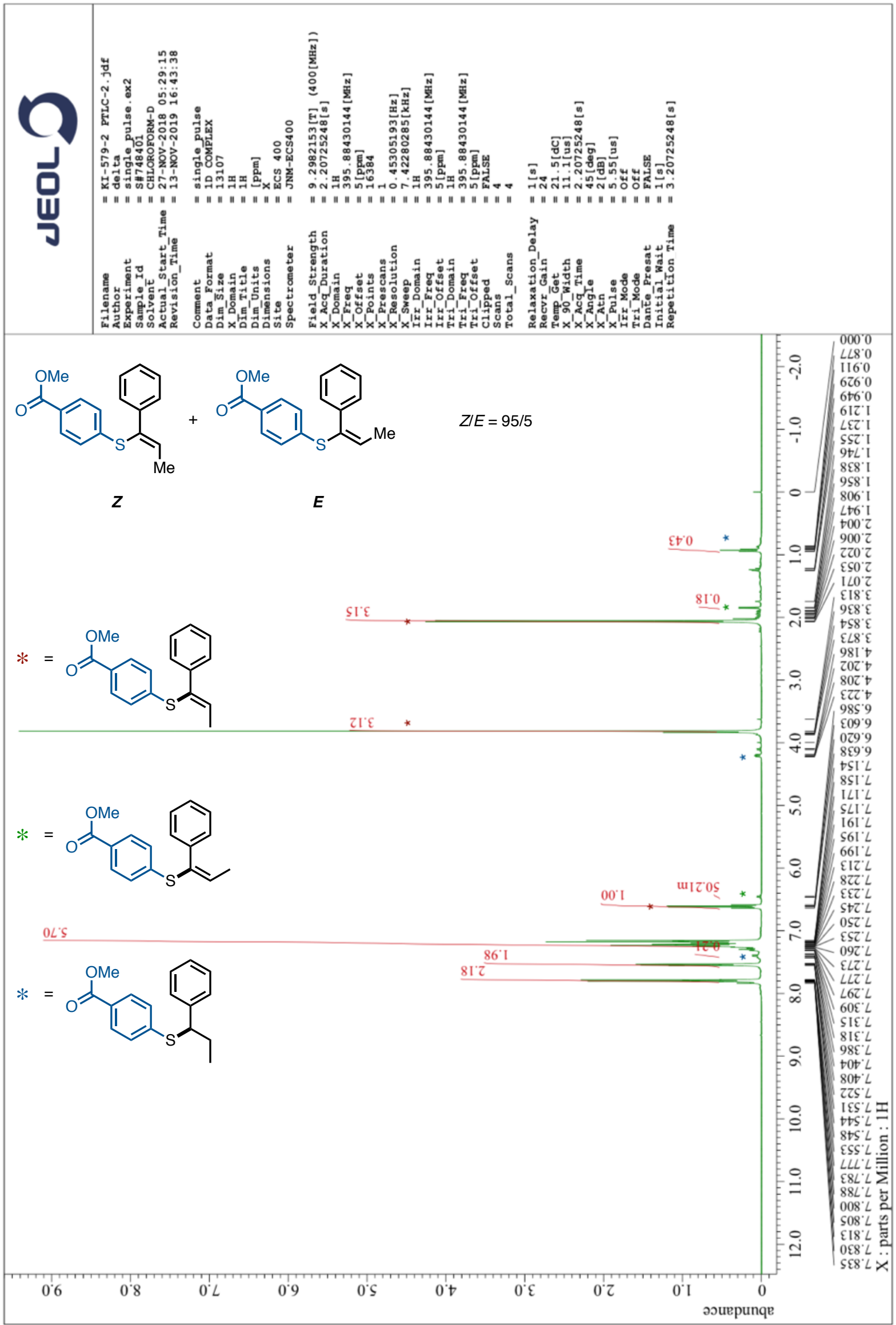


Supporting Information (Ishitobi, Muto, Yamaguchi) Pd-Catalyzed Alkenyl Thioether Synthesis from Thioesters and $\mathrm{N}$-Tosylhydrazones

${ }^{13} \mathrm{C}$ NMR of 3Ma (101 MHz, $\left.\mathrm{CDCl}_{3}\right)$

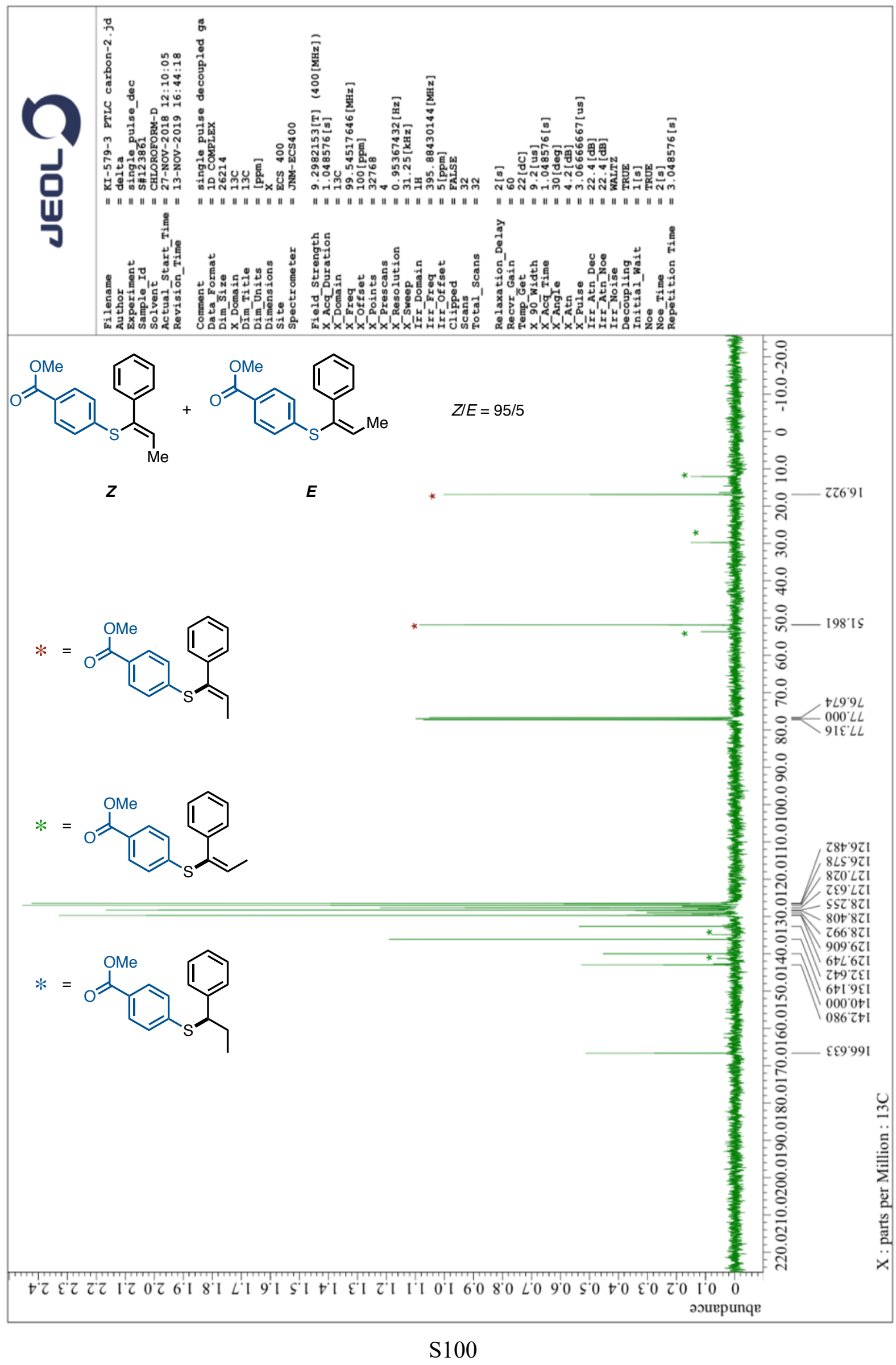


${ }^{1} \mathrm{H}$ NMR of $\mathbf{3 N a}\left(400 \mathrm{MHz}, \mathrm{CDCl}_{3}\right)$

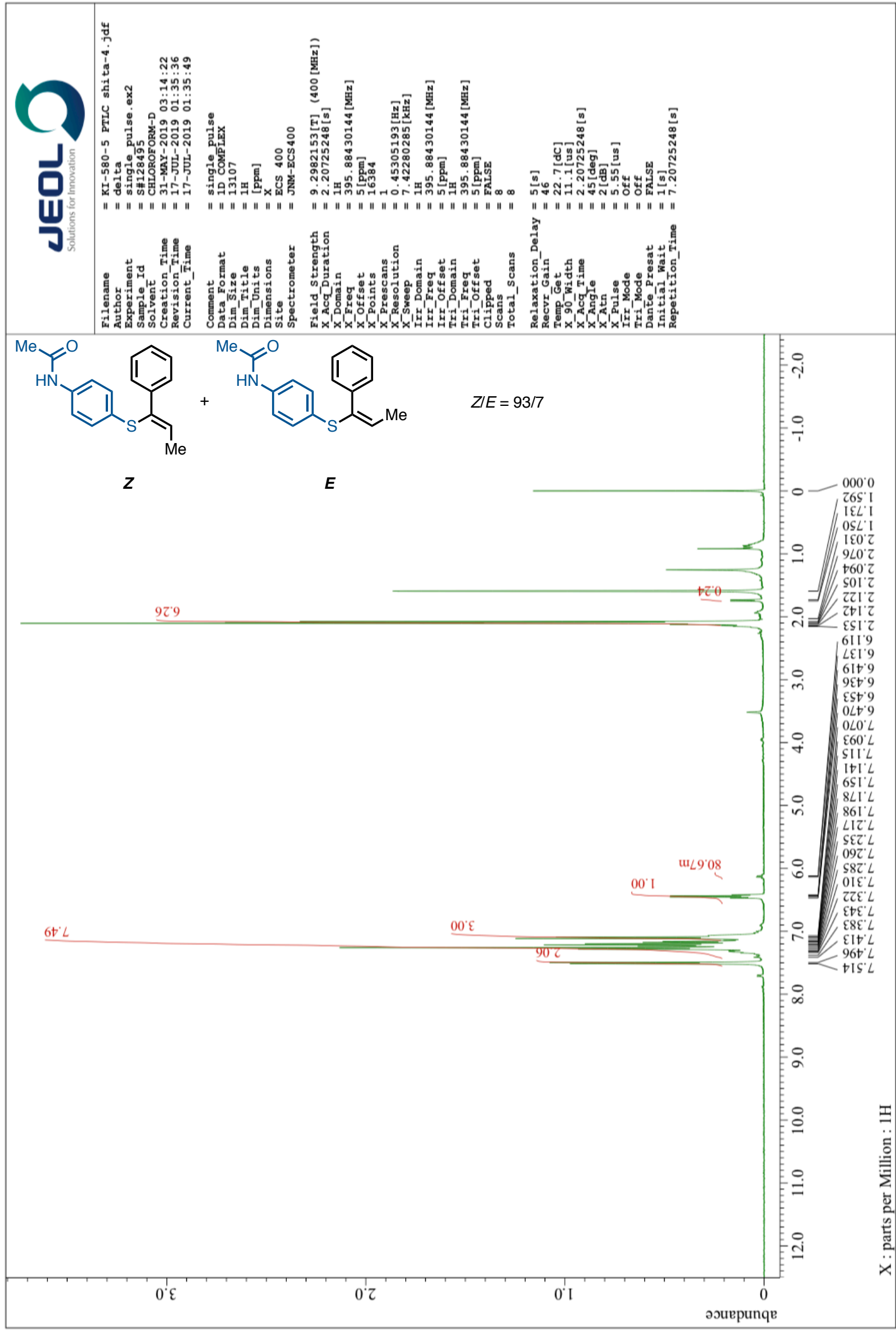


${ }^{13} \mathrm{C}$ NMR of $\mathbf{3 N a}\left(101 \mathrm{MHz}, \mathrm{CDCl}_{3}\right)$

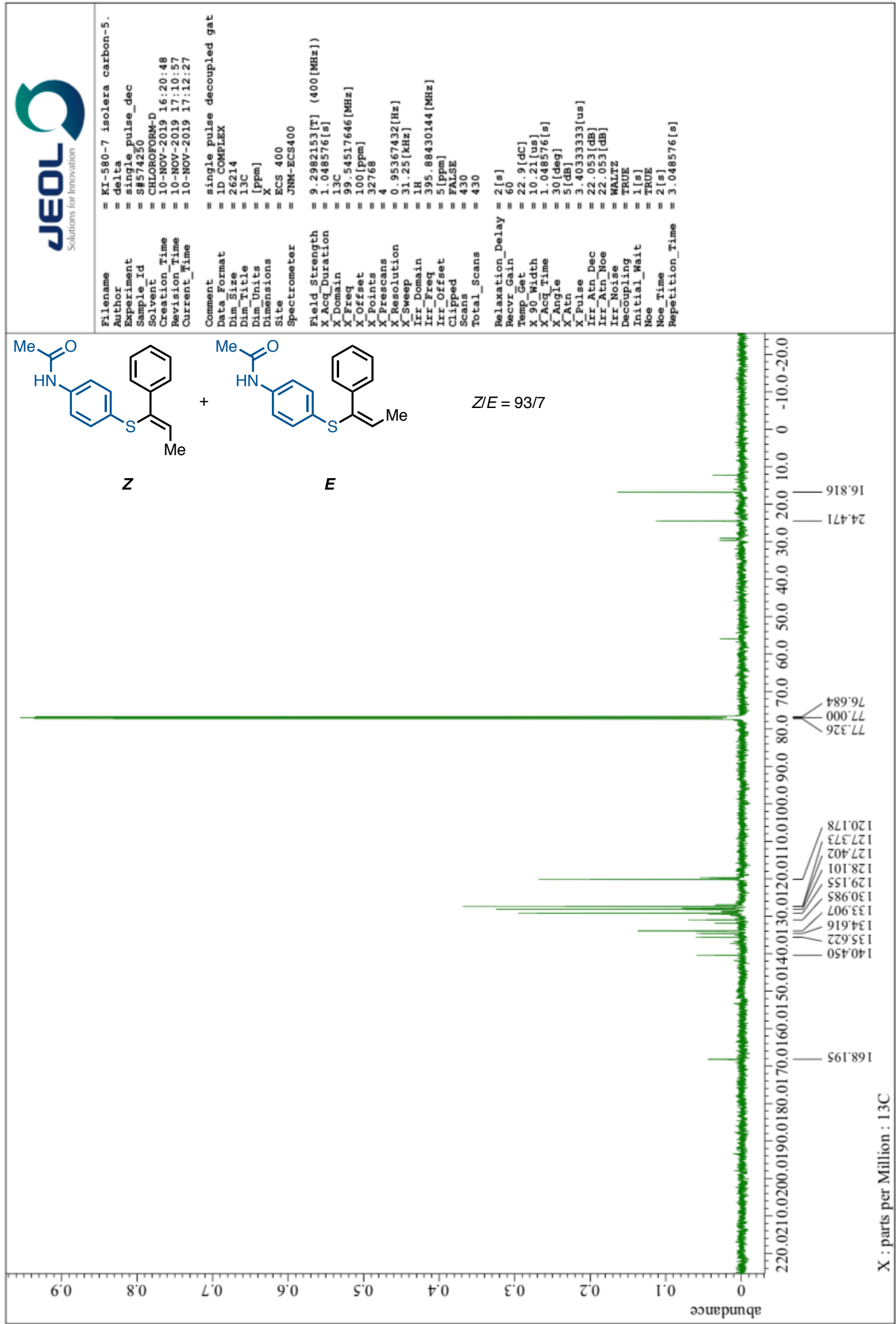


${ }^{1} \mathrm{H} \mathrm{NMR}$ of $\mathbf{3 O a}\left(400 \mathrm{MHz}, \mathrm{CDCl}_{3}\right)$

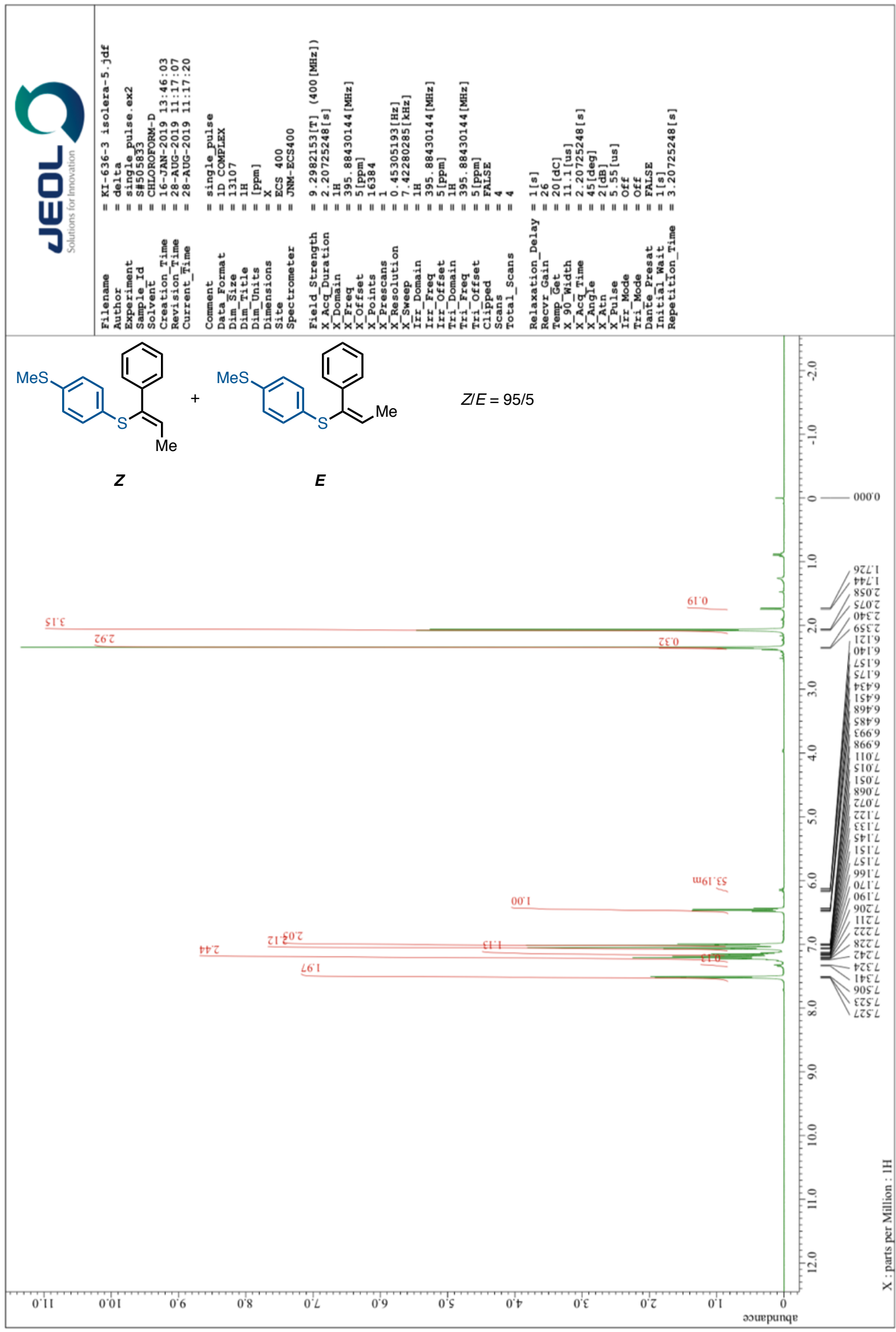


${ }^{13} \mathrm{C}$ NMR of 3Oa $\left(101 \mathrm{MHz}, \mathrm{CDCl}_{3}\right)$

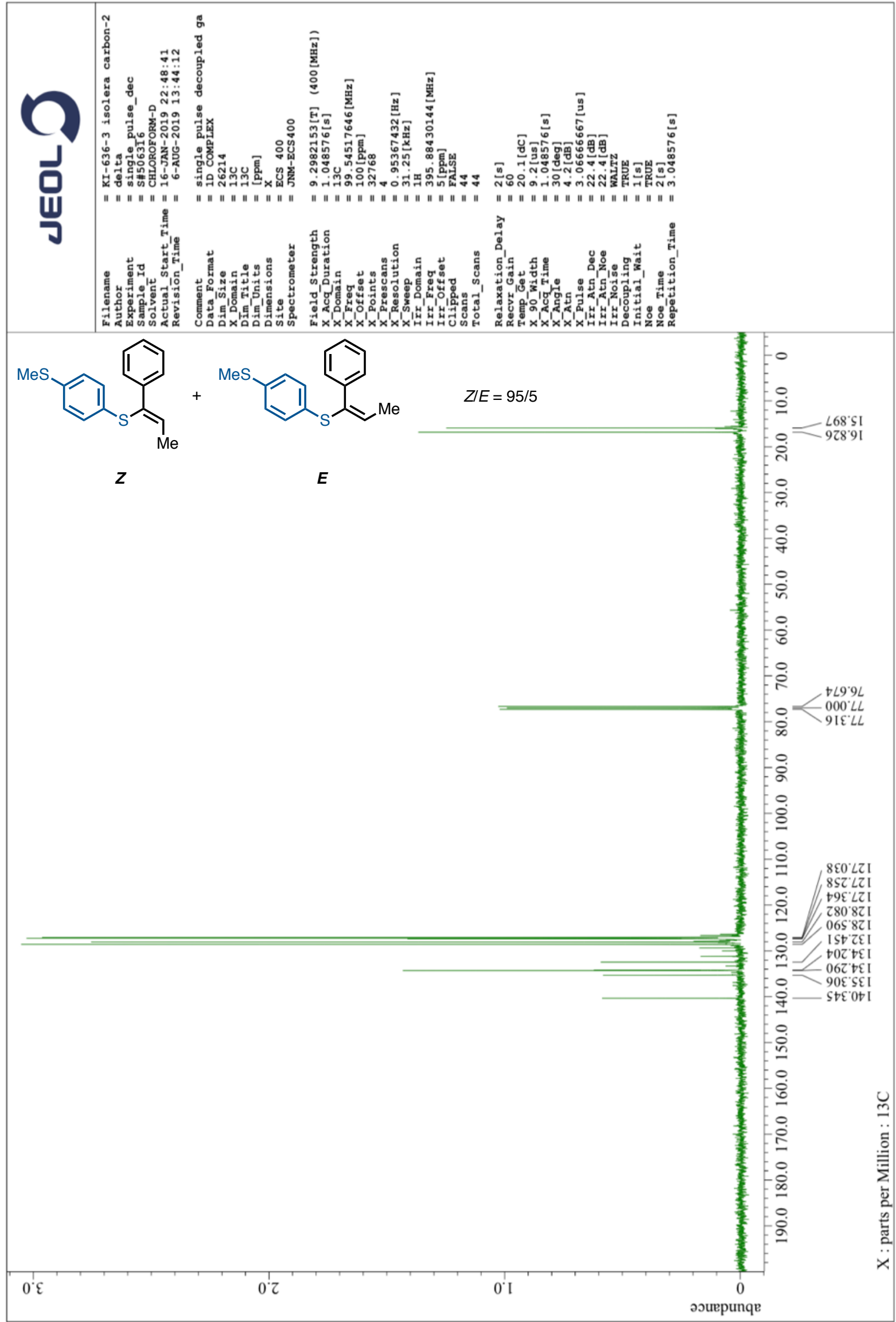


${ }^{1} \mathrm{H}$ NMR of $\mathbf{3 P a}\left(400 \mathrm{MHz}, \mathrm{CDCl}_{3}\right)$

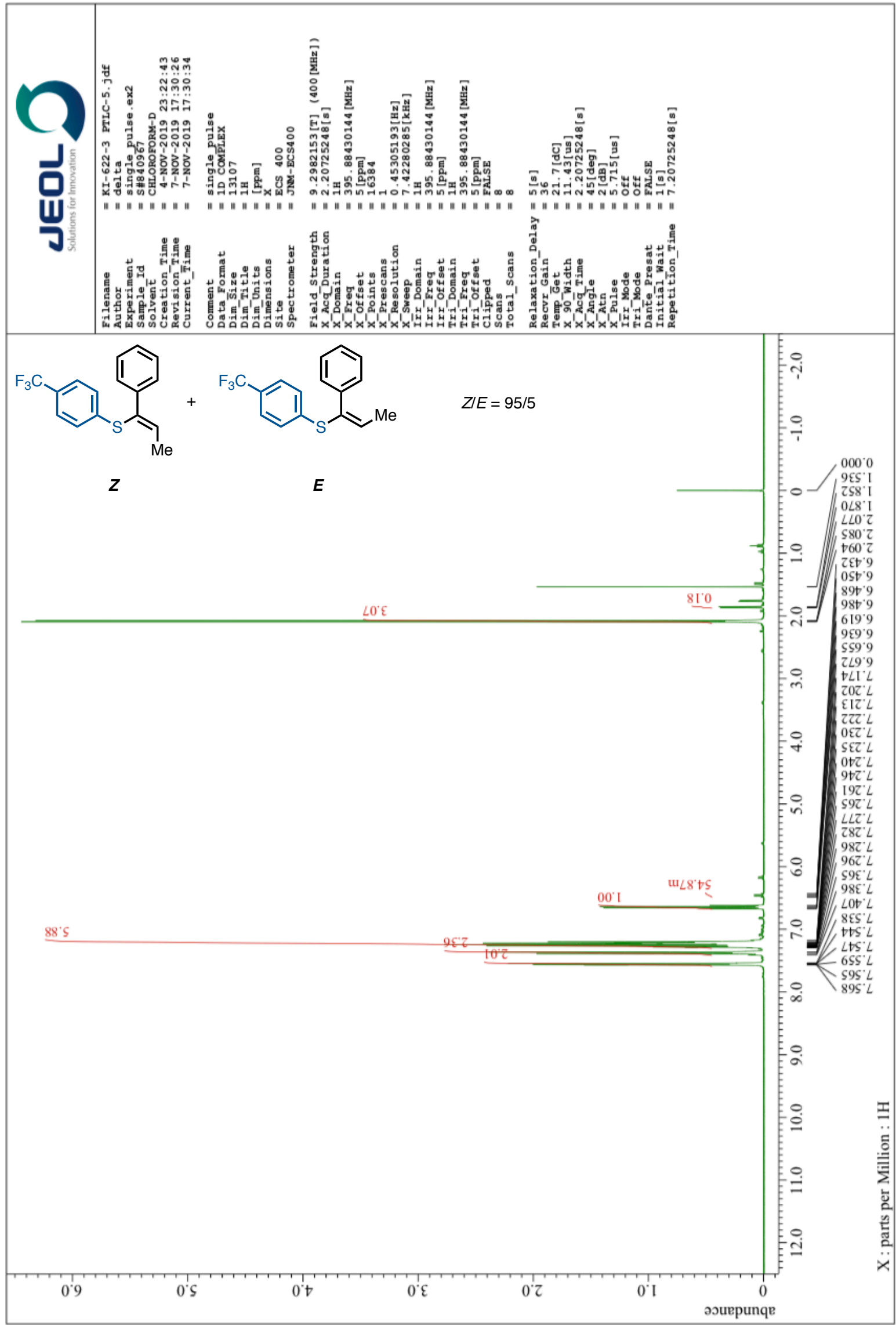


${ }^{13} \mathrm{C}$ NMR of 3Pa $\left(101 \mathrm{MHz}, \mathrm{CDCl}_{3}\right)$

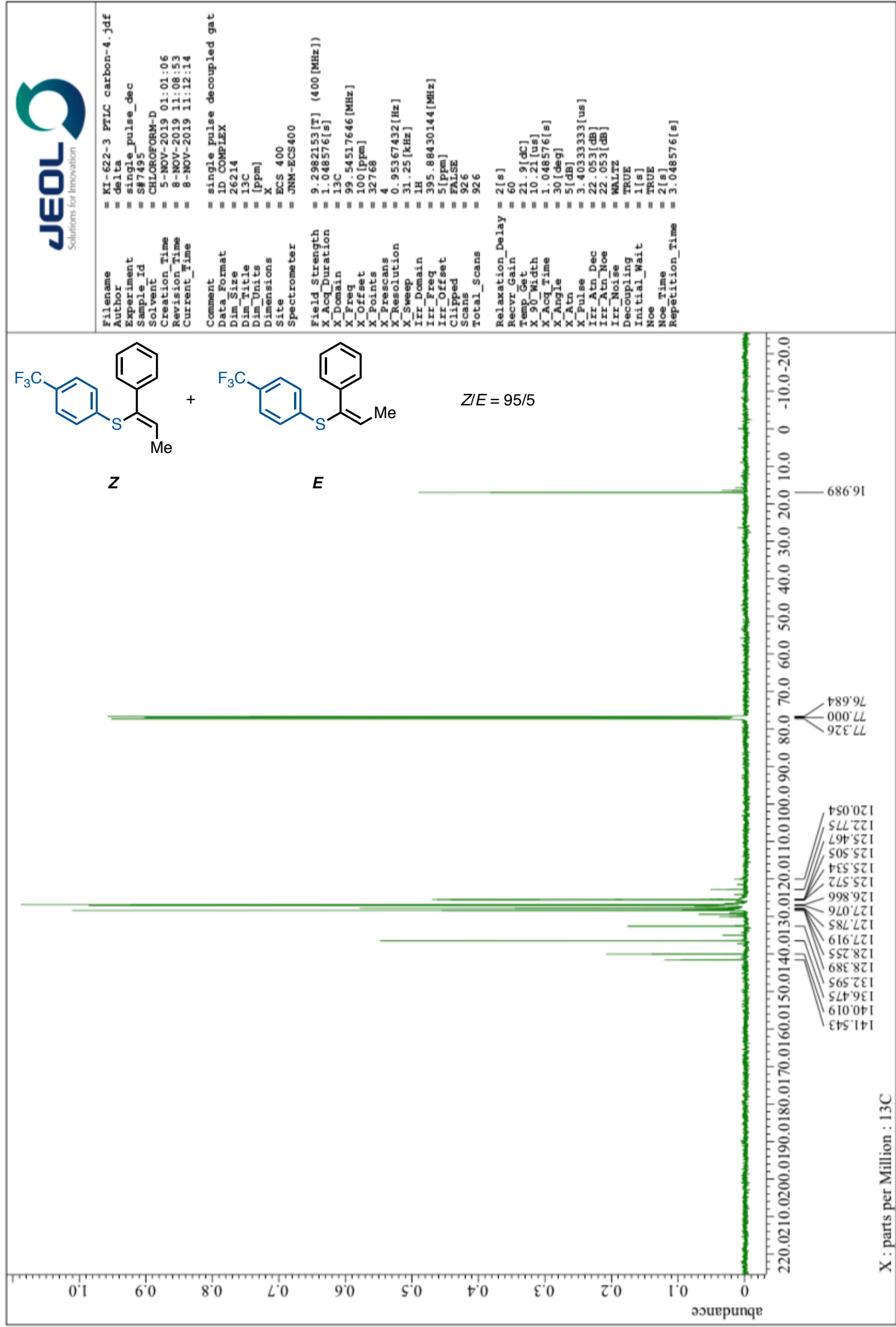


${ }^{1} \mathrm{H}$ NMR of 3Qa $\left(400 \mathrm{MHz}, \mathrm{CDCl}_{3}\right)$

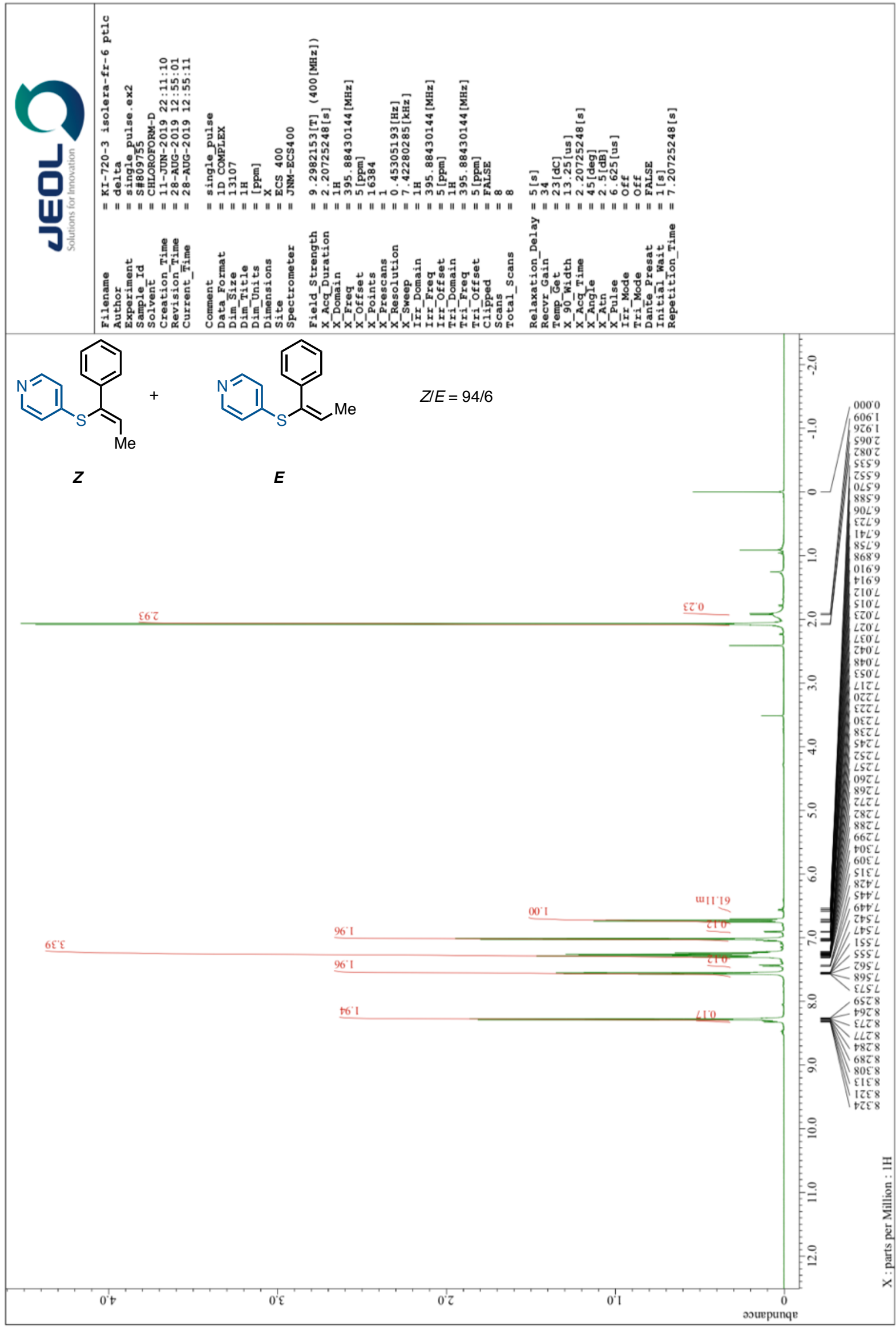


Supporting Information (Ishitobi, Muto, Yamaguchi) Pd-Catalyzed Alkenyl Thioether Synthesis from Thioesters and $\mathrm{N}$-Tosylhydrazones

${ }^{13} \mathrm{C}$ NMR of 3Qa (101 MHz, $\left.\mathrm{CDCl}_{3}\right)$

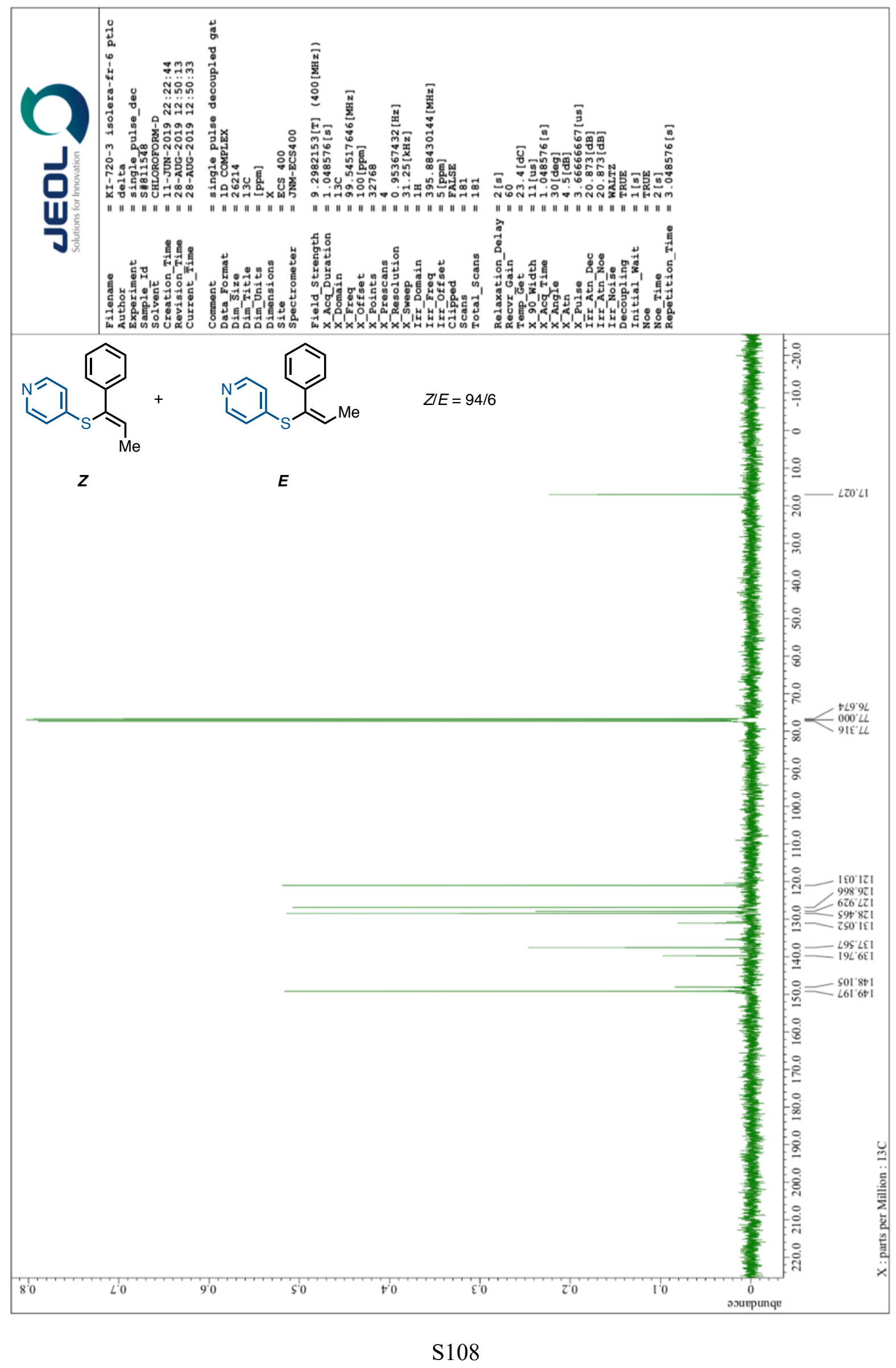


${ }^{1} \mathrm{H}$ NMR of $\mathbf{3 A b}\left(400 \mathrm{MHz}, \mathrm{CDCl}_{3}\right)$

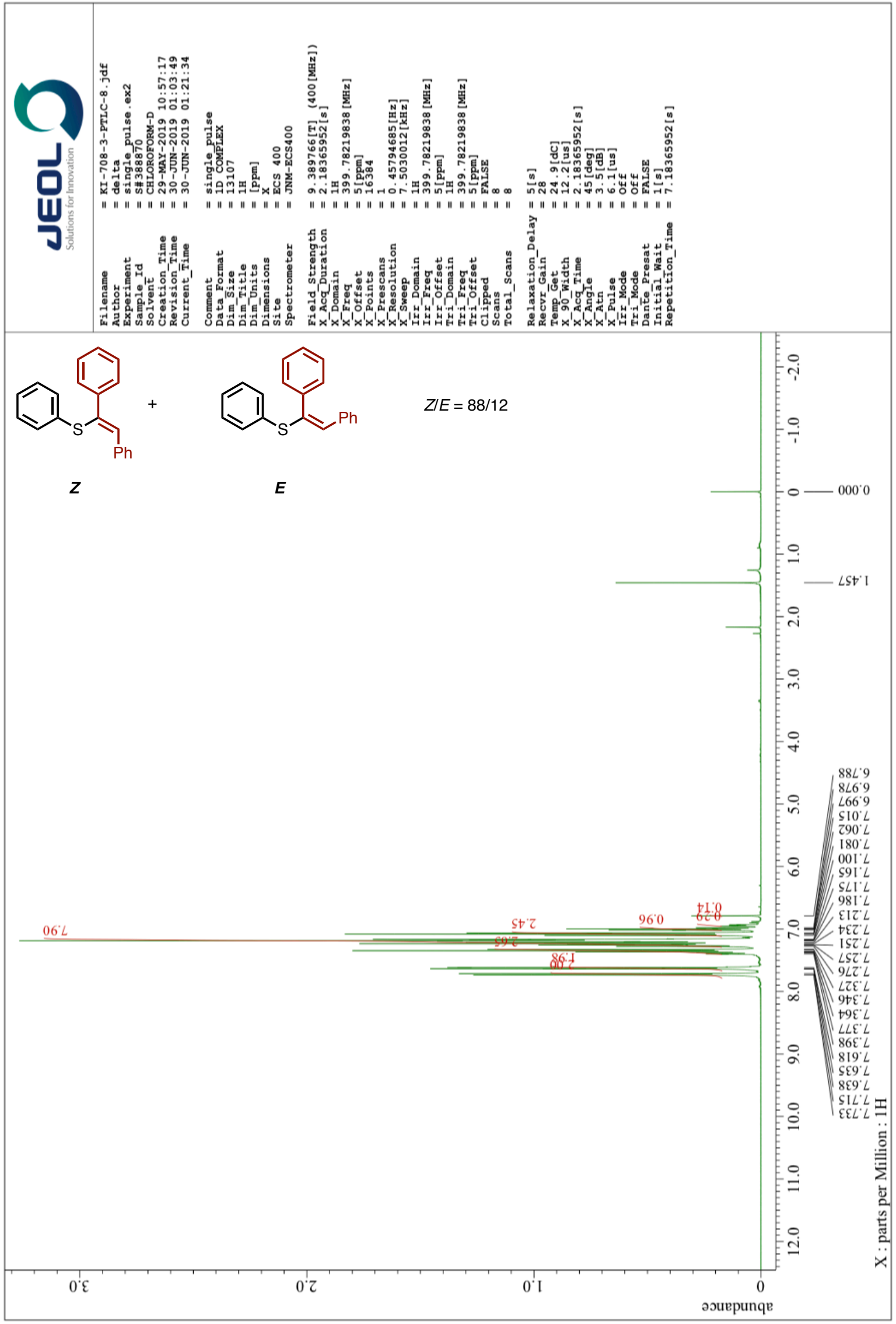


${ }^{13} \mathrm{C}$ NMR of $\mathbf{3 A b}\left(101 \mathrm{MHz}, \mathrm{CDCl}_{3}\right)$

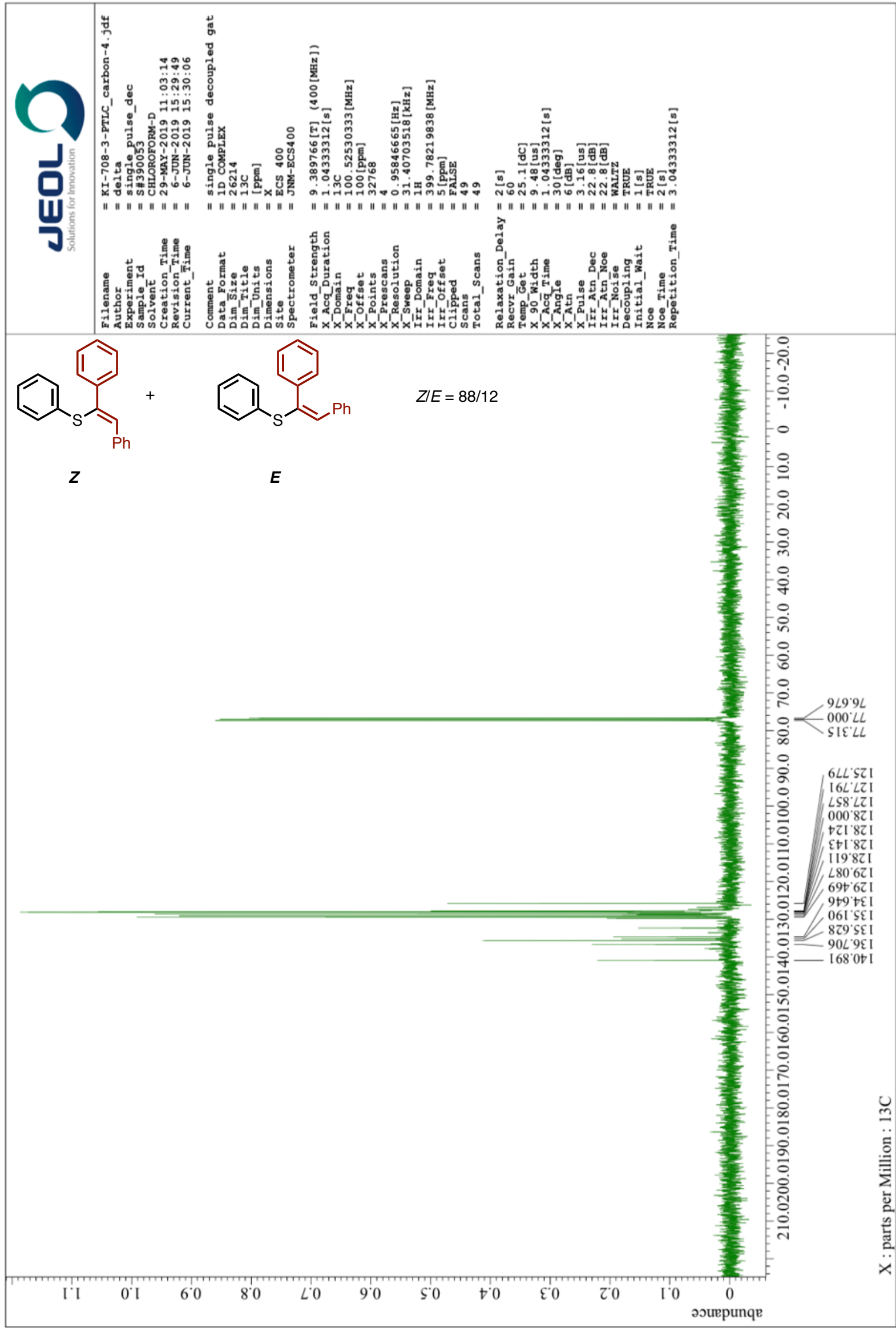


${ }^{1} \mathrm{H}$ NMR of $3 \mathrm{Ac}\left(400 \mathrm{MHz}, \mathrm{CDCl}_{3}\right)$

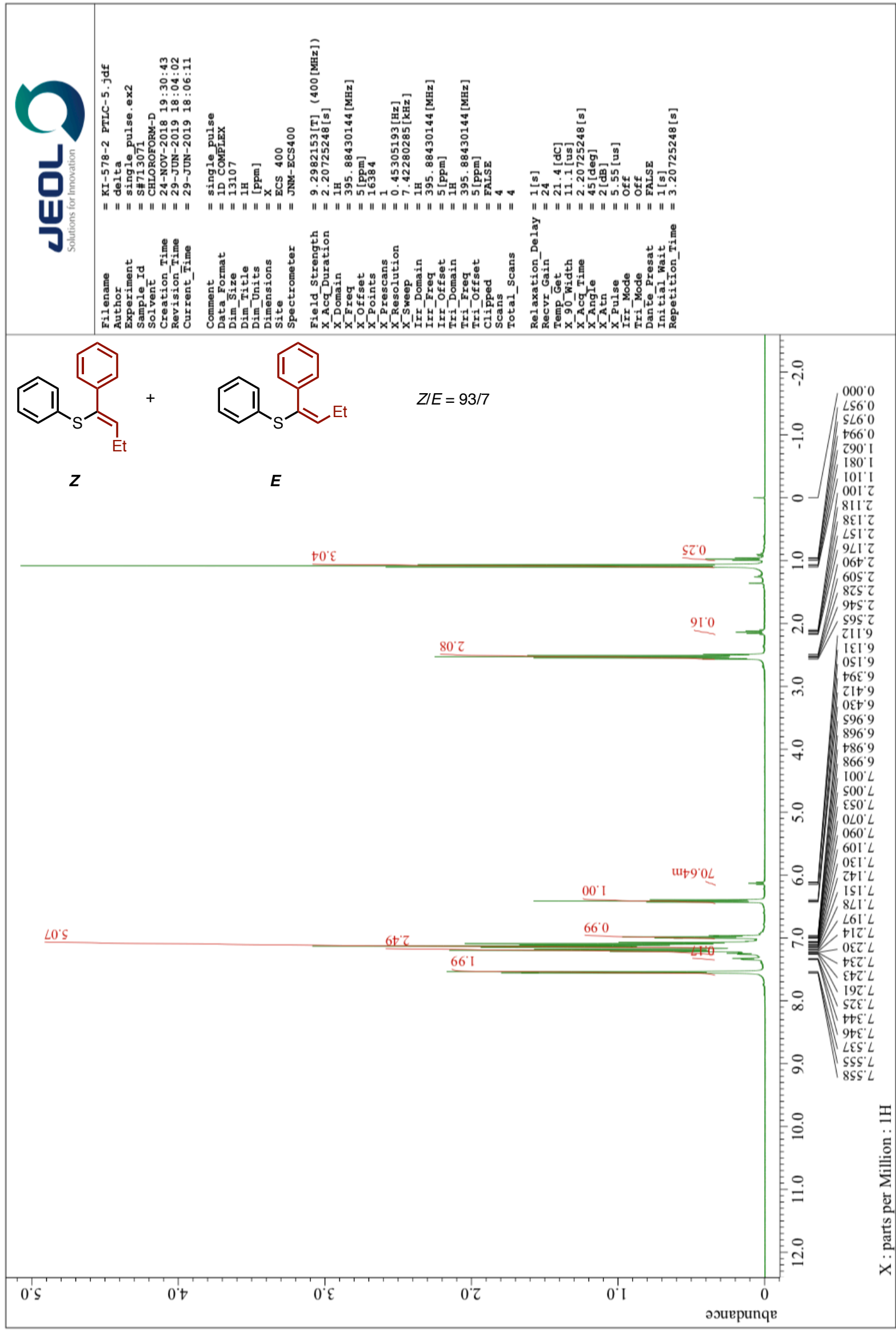


${ }^{13} \mathrm{C}$ NMR of $\mathbf{3 A c}\left(101 \mathrm{MHz}, \mathrm{CDCl}_{3}\right)$

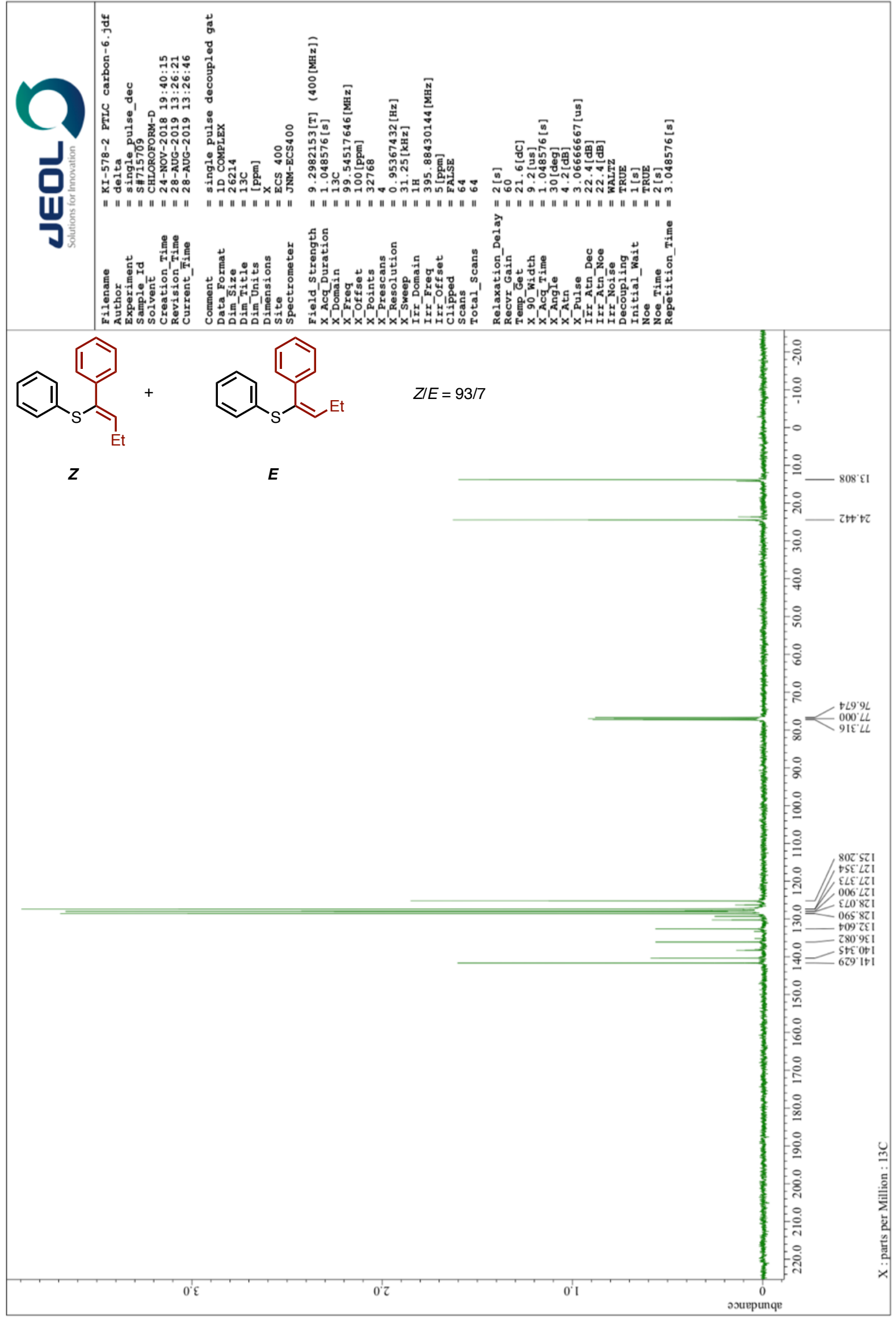


${ }^{1} \mathrm{H}$ NMR of 3Ad $\left(400 \mathrm{MHz}, \mathrm{CDCl}_{3}\right)$

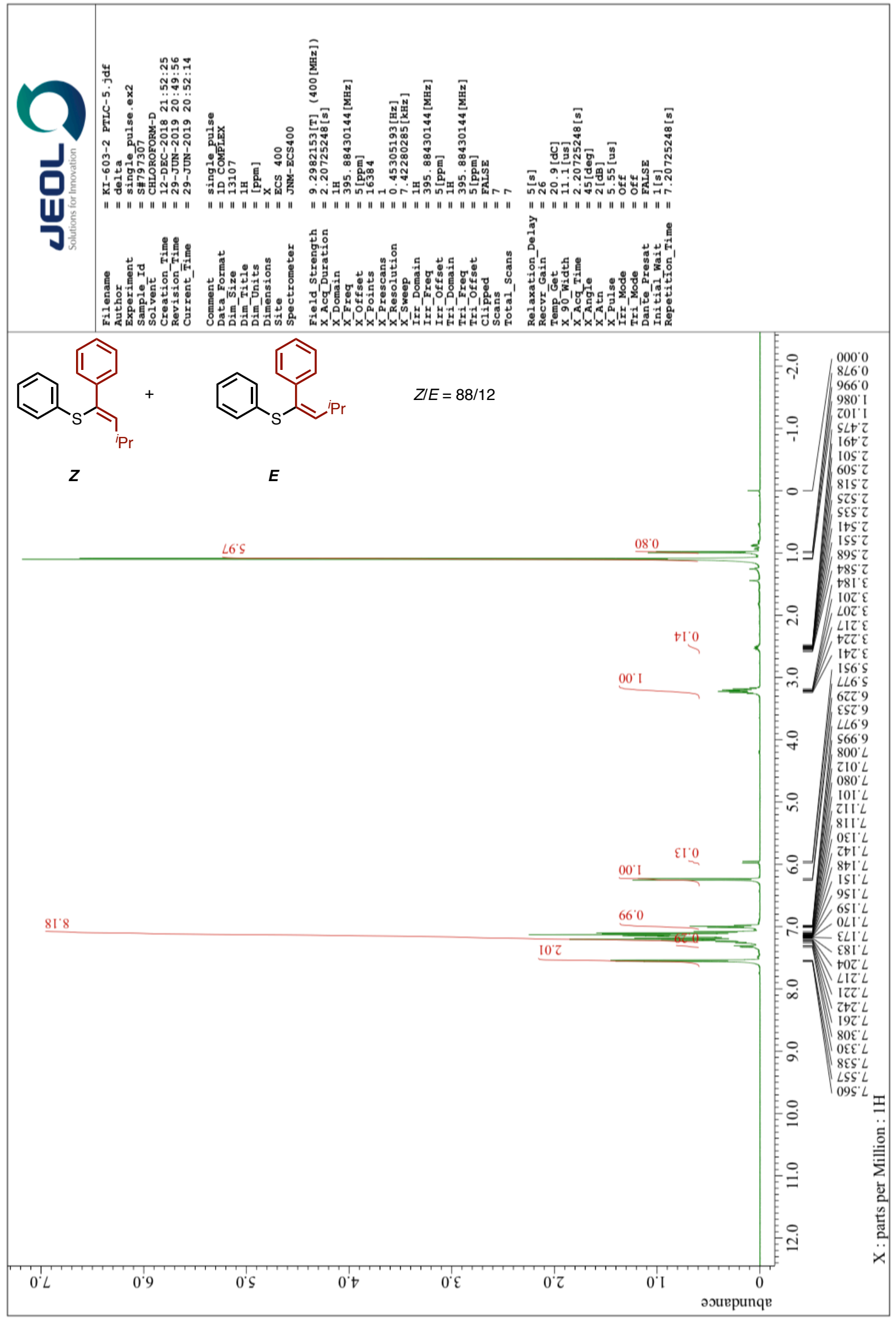


Supporting Information (Ishitobi, Muto, Yamaguchi) Pd-Catalyzed Alkenyl Thioether Synthesis from Thioesters and N-Tosylhydrazones

${ }^{13} \mathrm{C}$ NMR of 3Ad (101 MHz, $\left.\mathrm{CDCl}_{3}\right)$

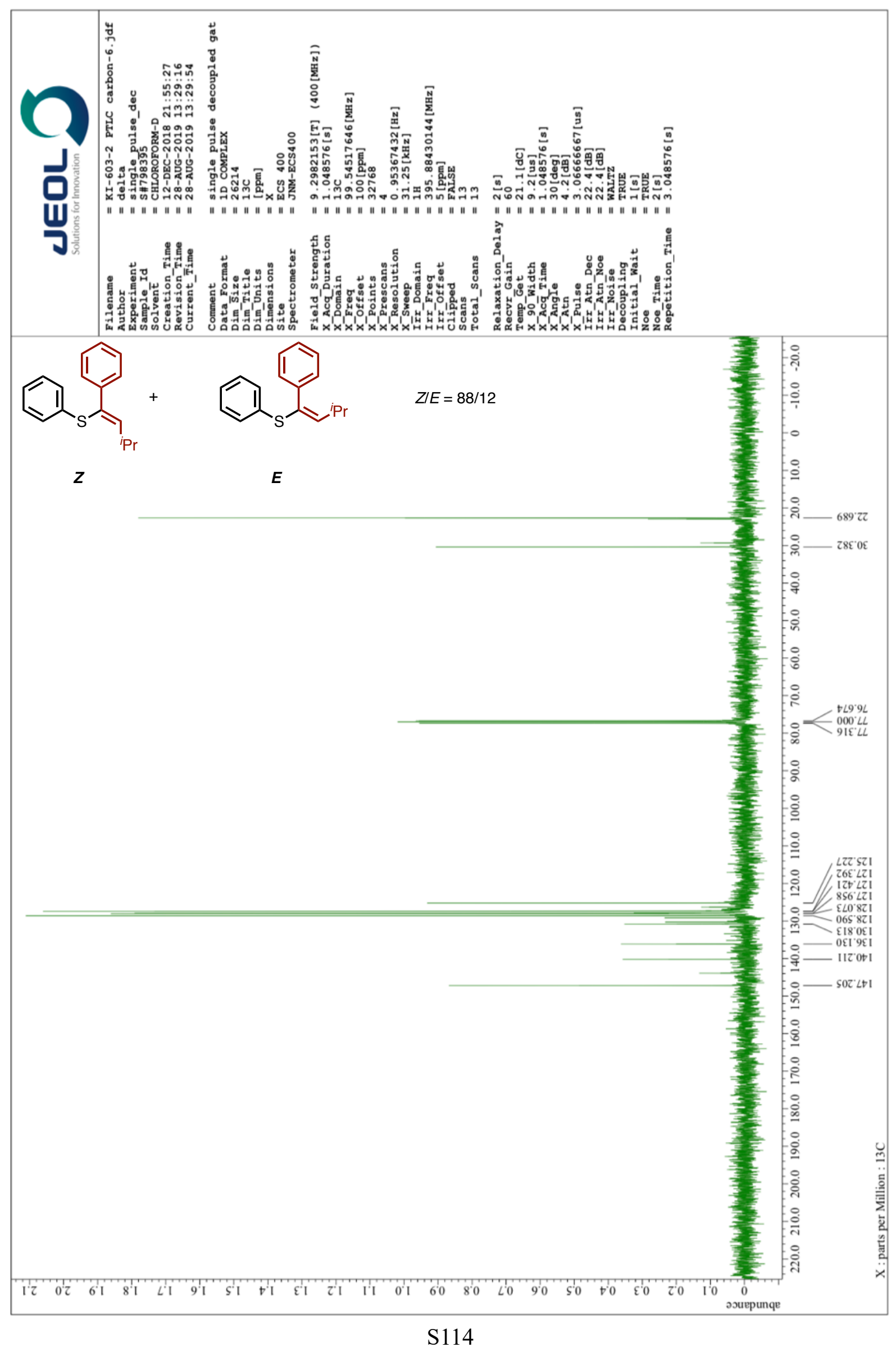


${ }^{1} \mathrm{H}$ NMR of $3 \mathrm{Ae}\left(400 \mathrm{MHz}, \mathrm{CDCl}_{3}\right)$

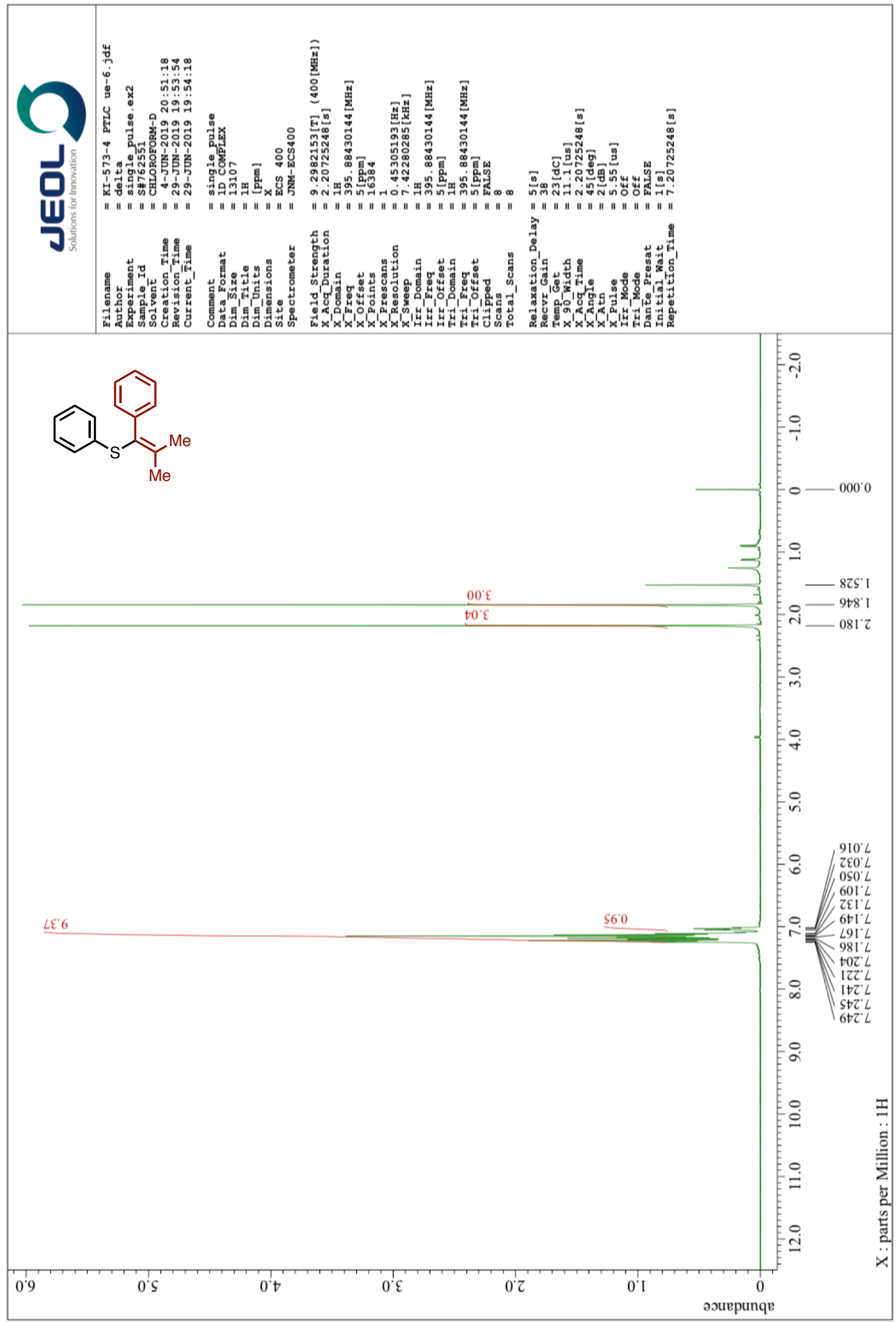


${ }^{13} \mathrm{C}$ NMR of $3 \mathrm{Ae}\left(101 \mathrm{MHz}, \mathrm{CDCl}_{3}\right)$

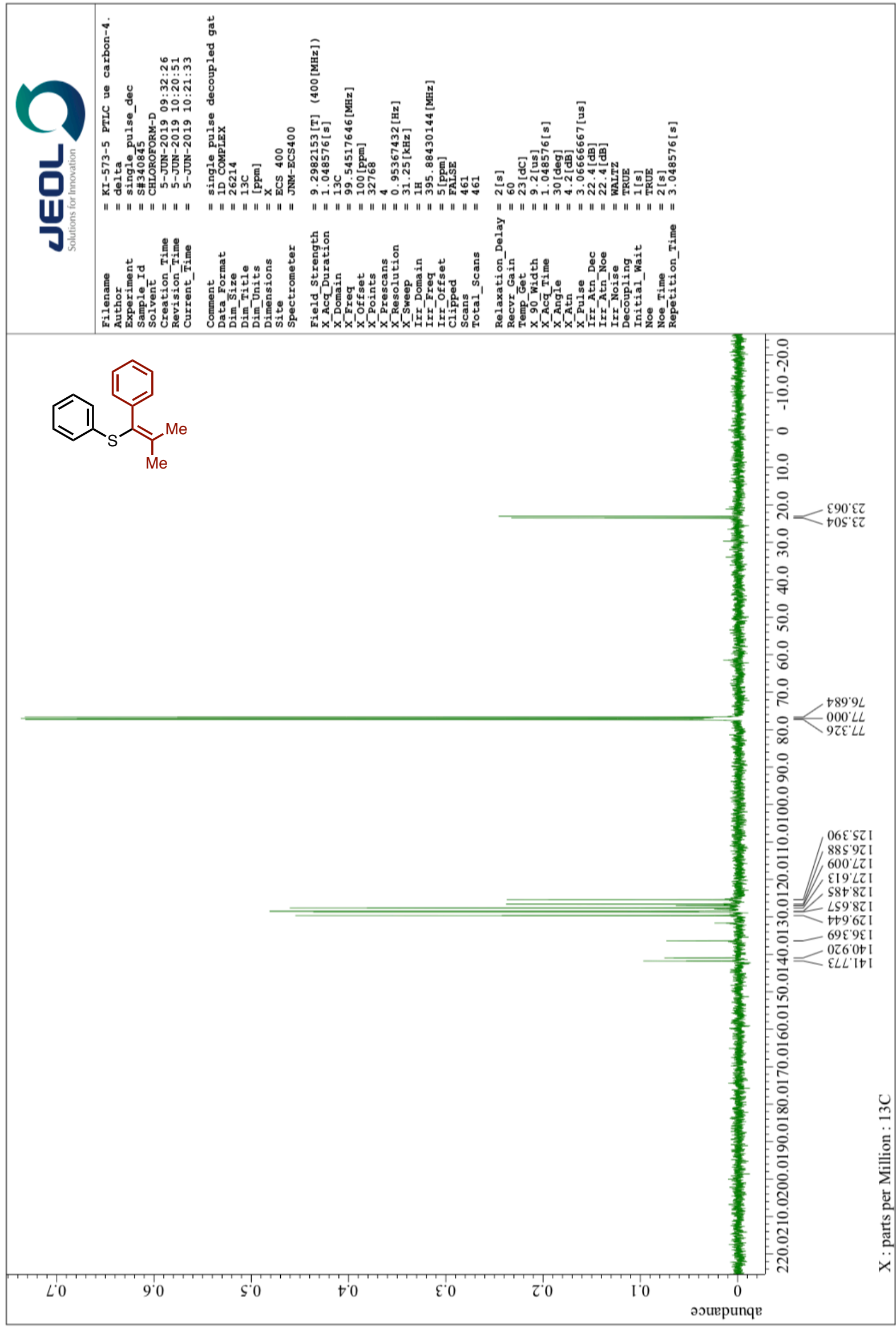


${ }^{1} \mathrm{H}$ NMR of $\mathbf{3 A f}\left(400 \mathrm{MHz}, \mathrm{CDCl}_{3}\right)$

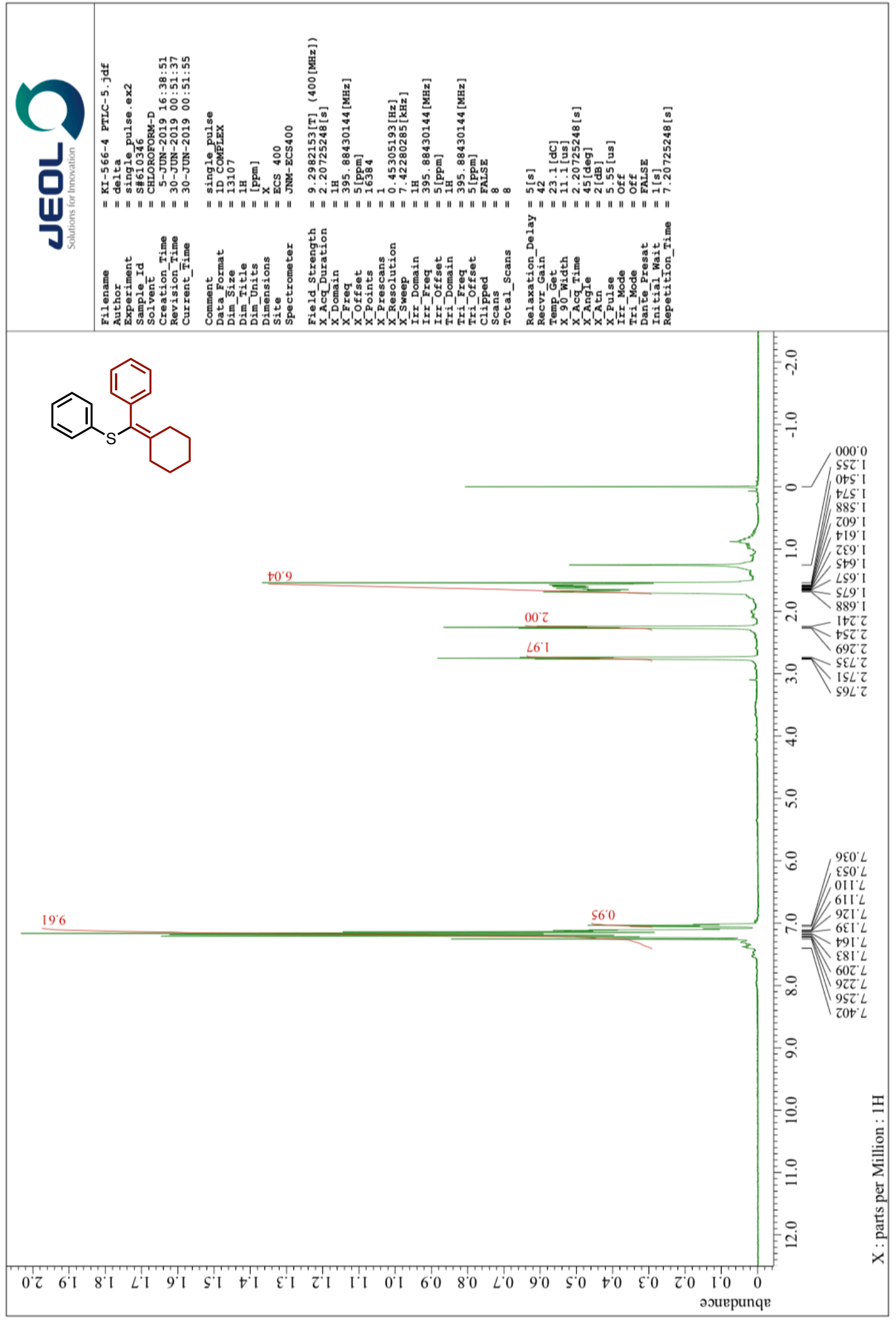


${ }^{13} \mathrm{C}$ NMR of $3 \mathbf{A f}\left(101 \mathrm{MHz}, \mathrm{CDCl}_{3}\right)$

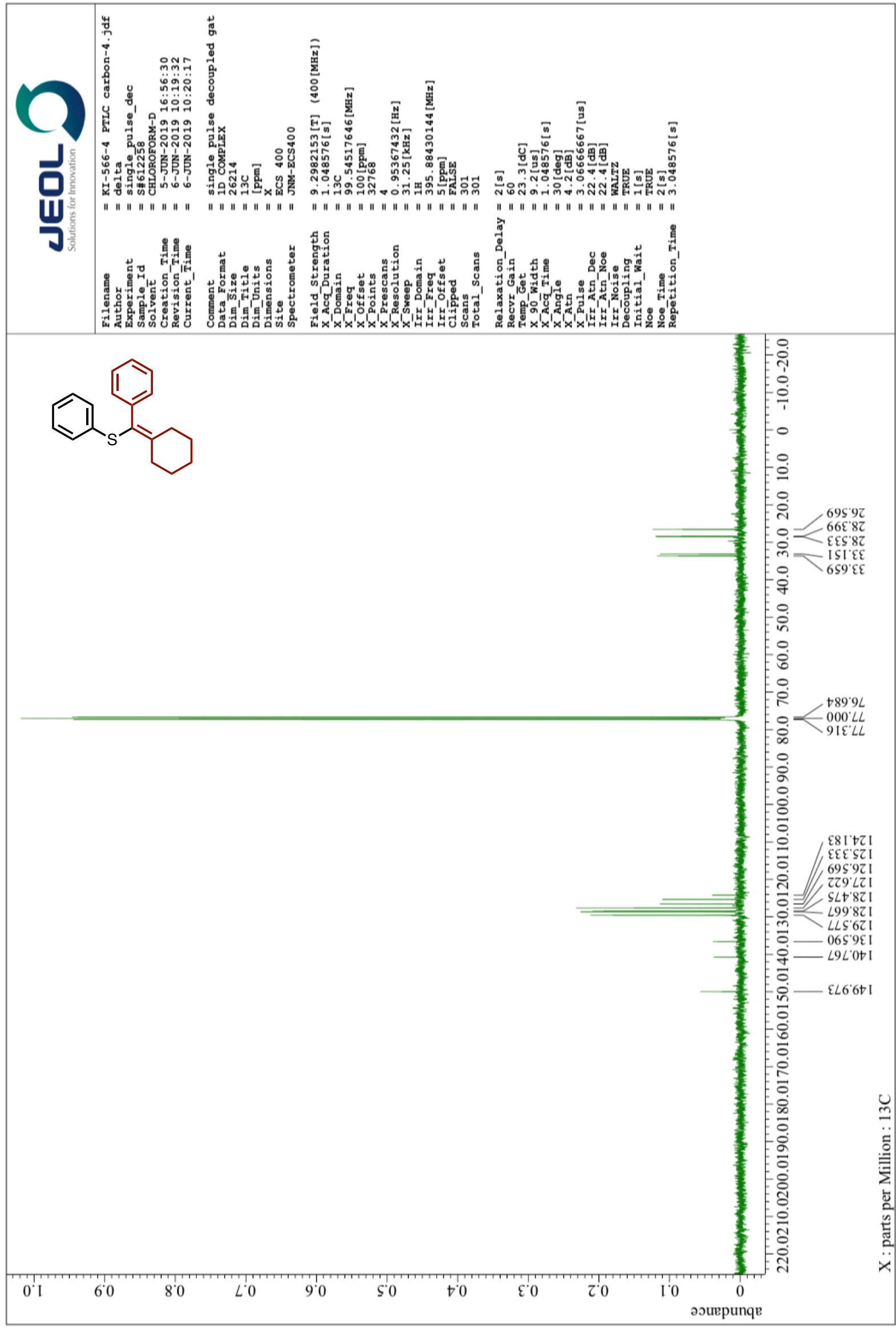


${ }^{1} \mathrm{H}$ NMR of $\mathbf{3 A g}\left(400 \mathrm{MHz}, \mathrm{CDCl}_{3}\right)$

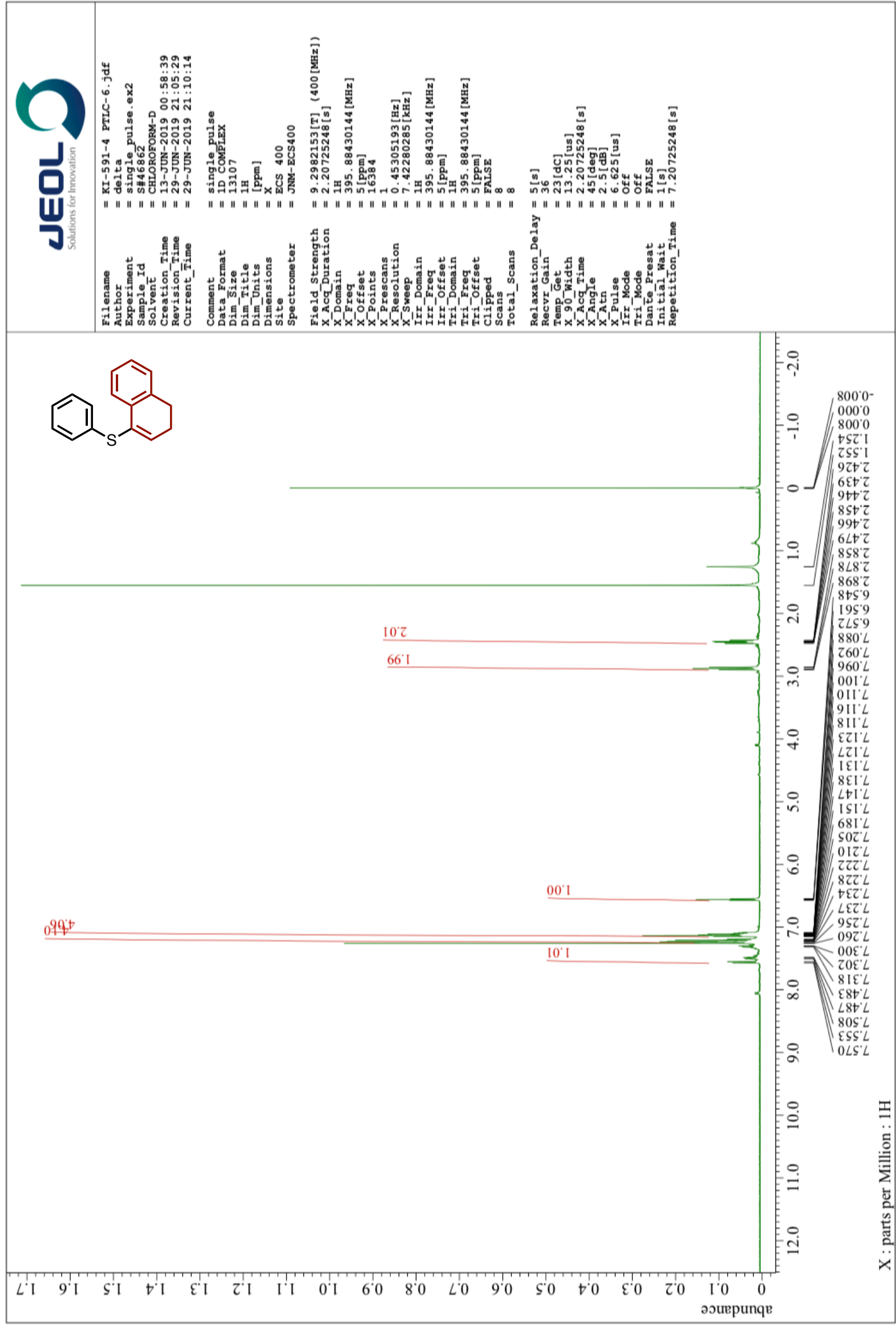


${ }^{13} \mathrm{C}$ NMR of $\mathbf{3 A g}\left(101 \mathrm{MHz}, \mathrm{CDCl}_{3}\right)$

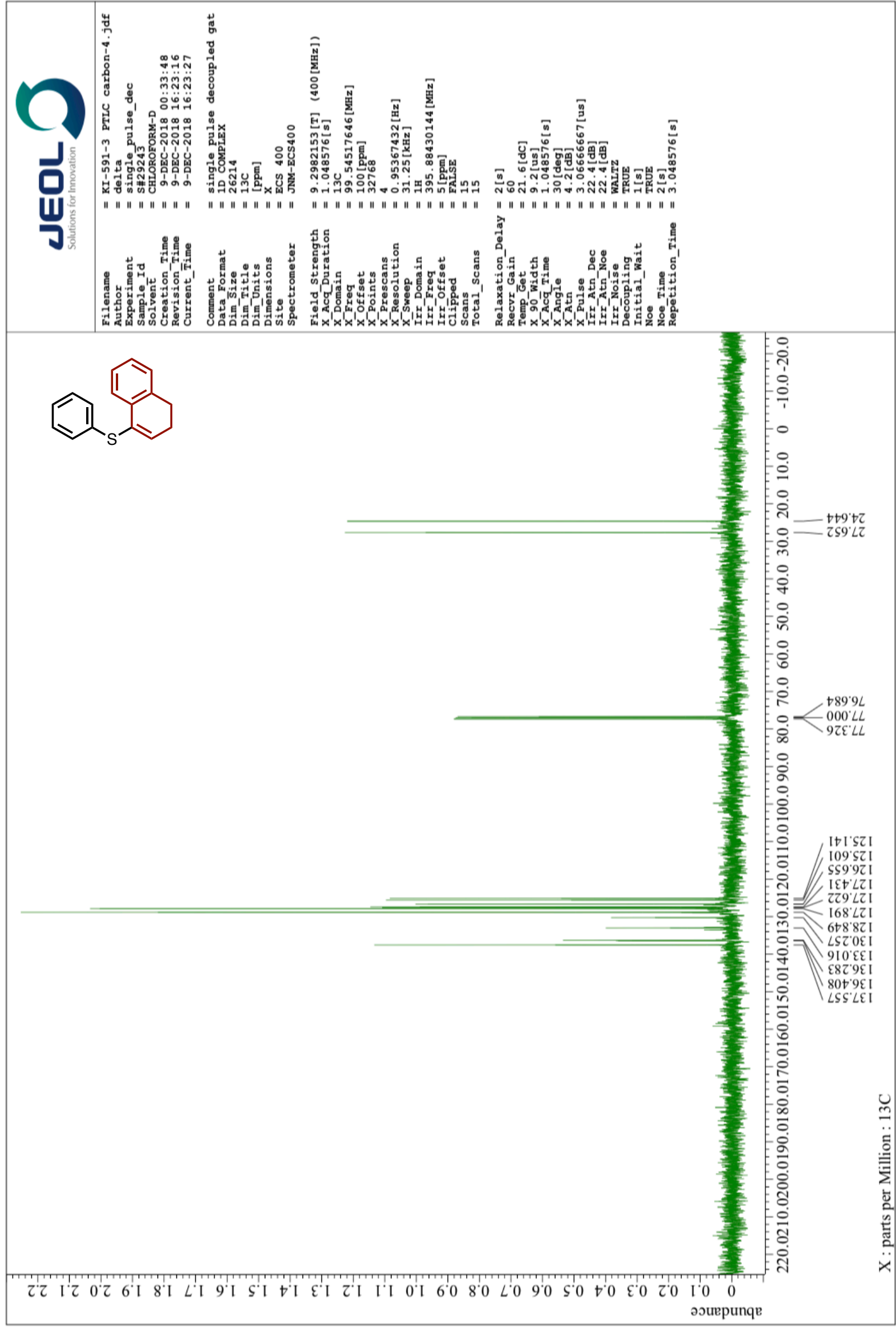


${ }^{1} \mathrm{H}$ NMR of $\mathbf{3 A h}\left(400 \mathrm{MHz}, \mathrm{CDCl}_{3}\right)$

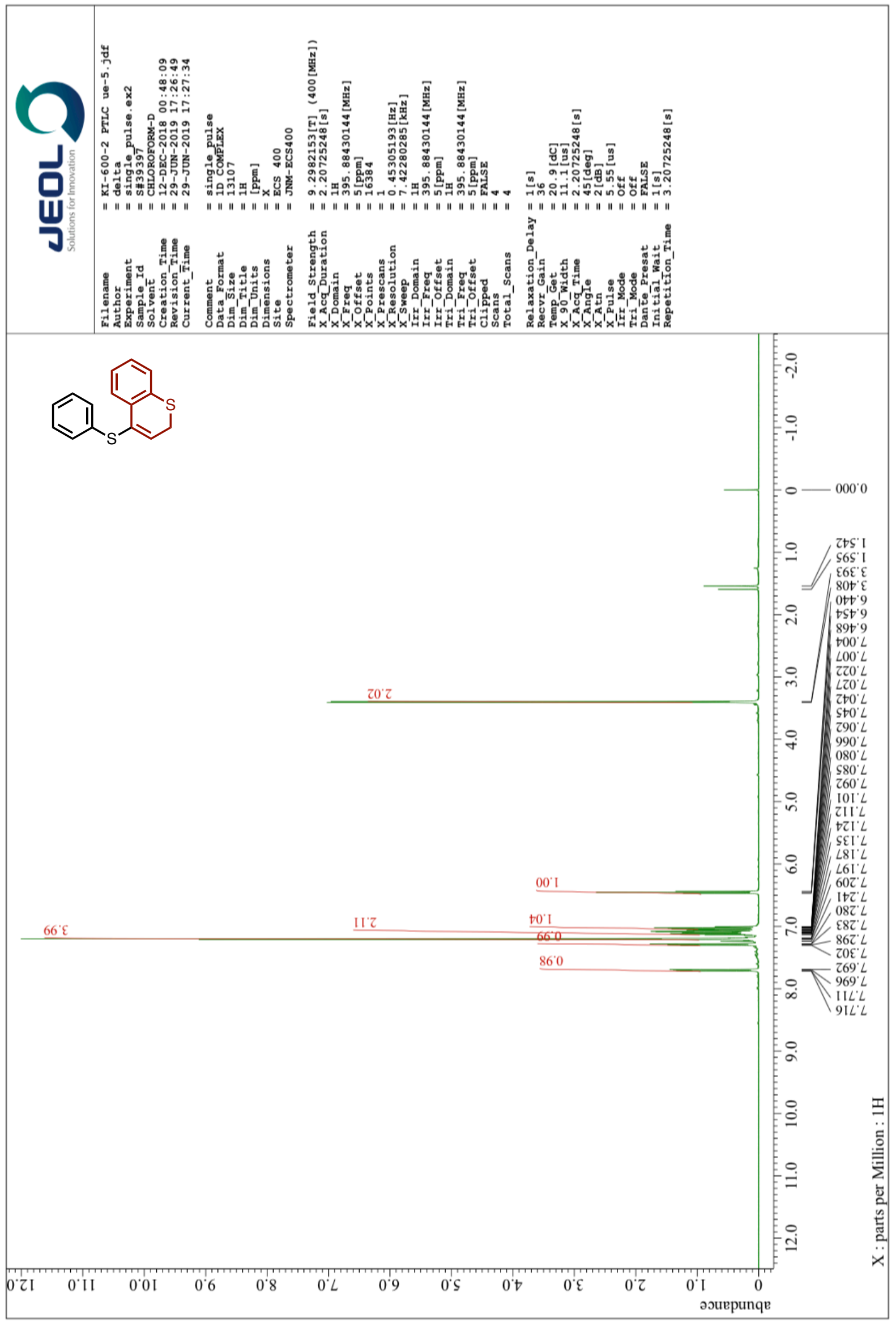


${ }^{13} \mathrm{C}$ NMR of $\mathbf{3 A h}\left(101 \mathrm{MHz}, \mathrm{CDCl}_{3}\right)$

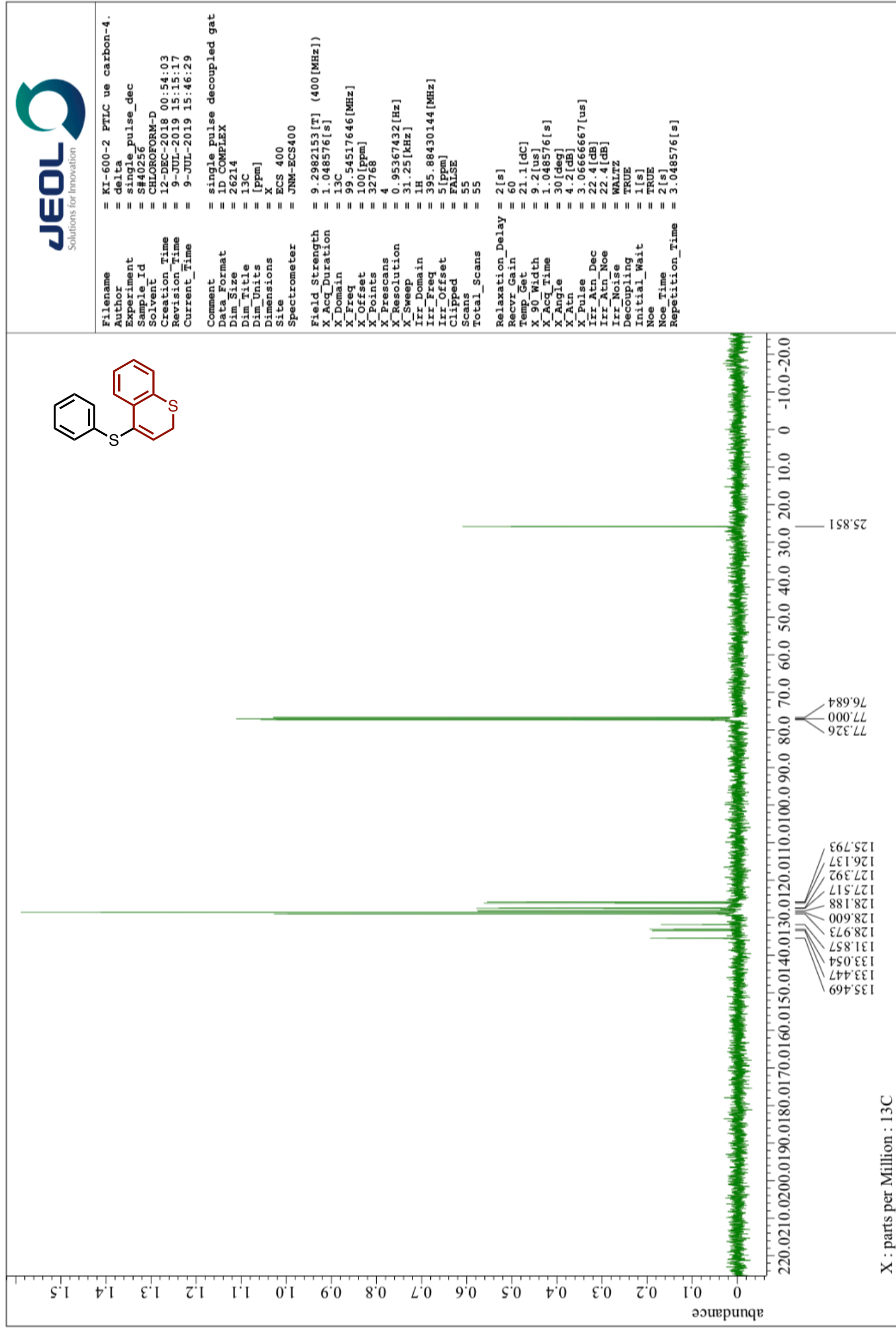


${ }^{1} \mathrm{H}$ NMR of $\mathbf{3 A i}\left(400 \mathrm{MHz}, \mathrm{CDCl}_{3}\right)$

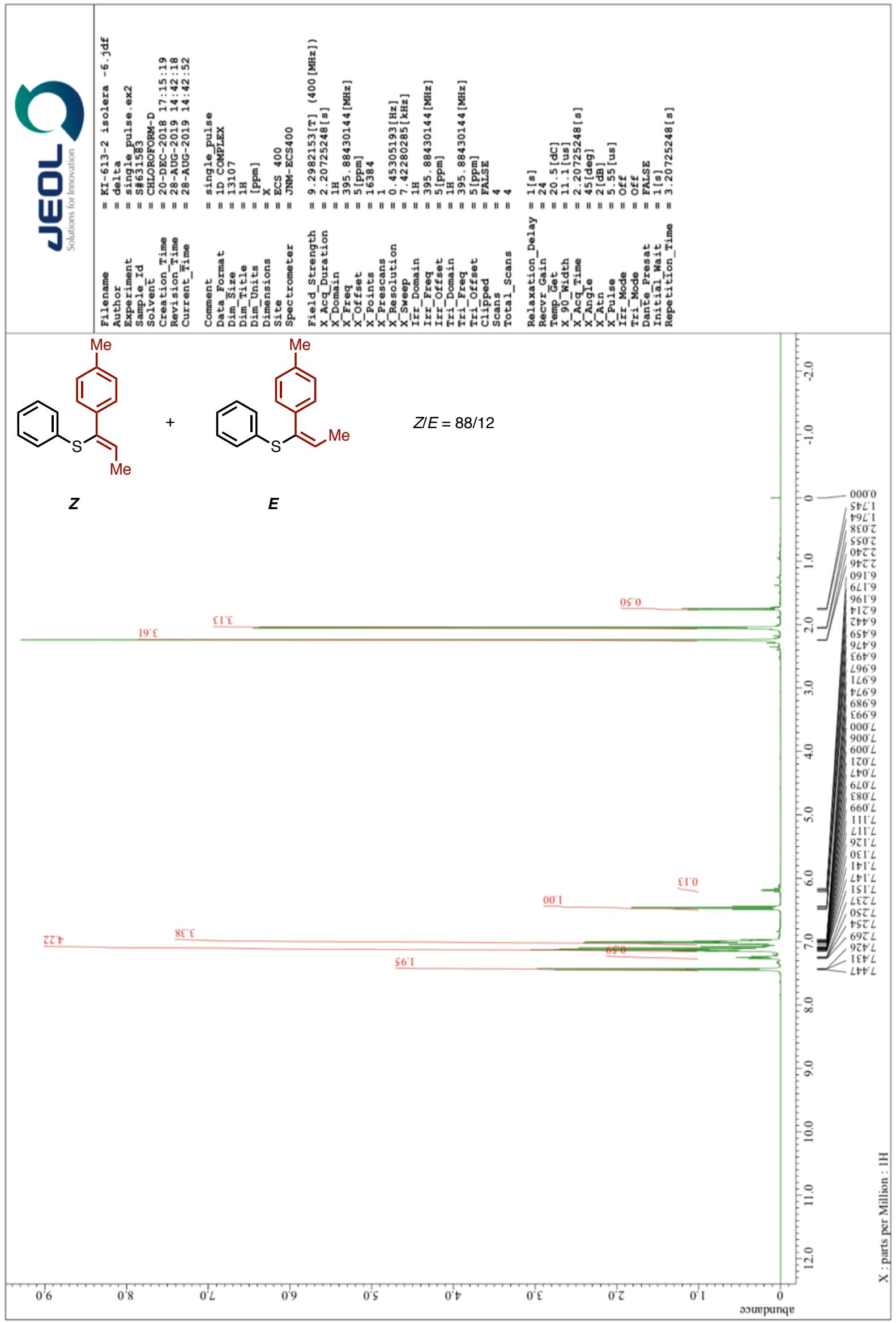


${ }^{13} \mathrm{C}$ NMR of $3 \mathrm{Ai}\left(101 \mathrm{MHz}, \mathrm{CDCl}_{3}\right)$

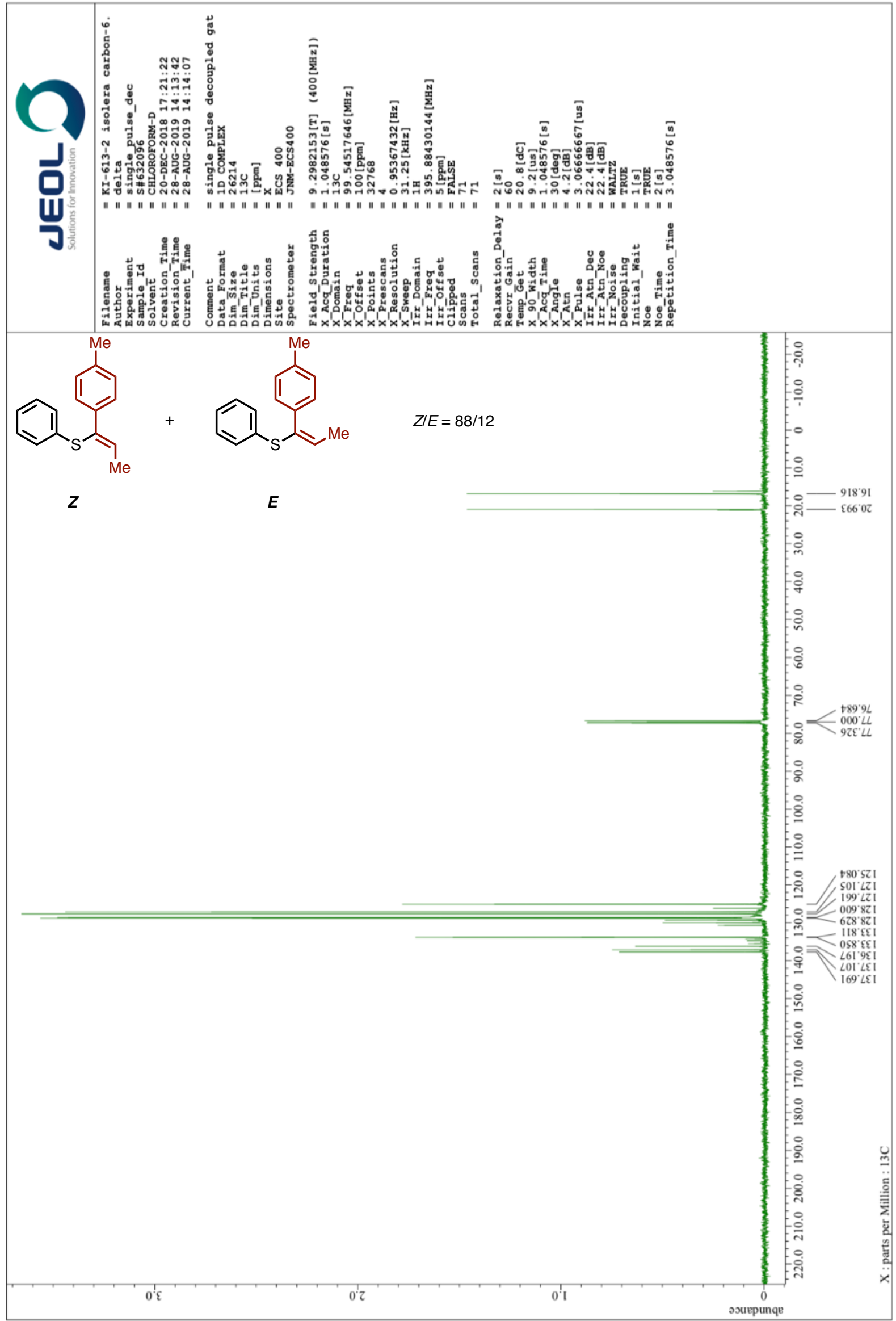


${ }^{1} \mathrm{H}$ NMR of $\mathbf{3 A j}\left(400 \mathrm{MHz}, \mathrm{CDCl}_{3}\right)$

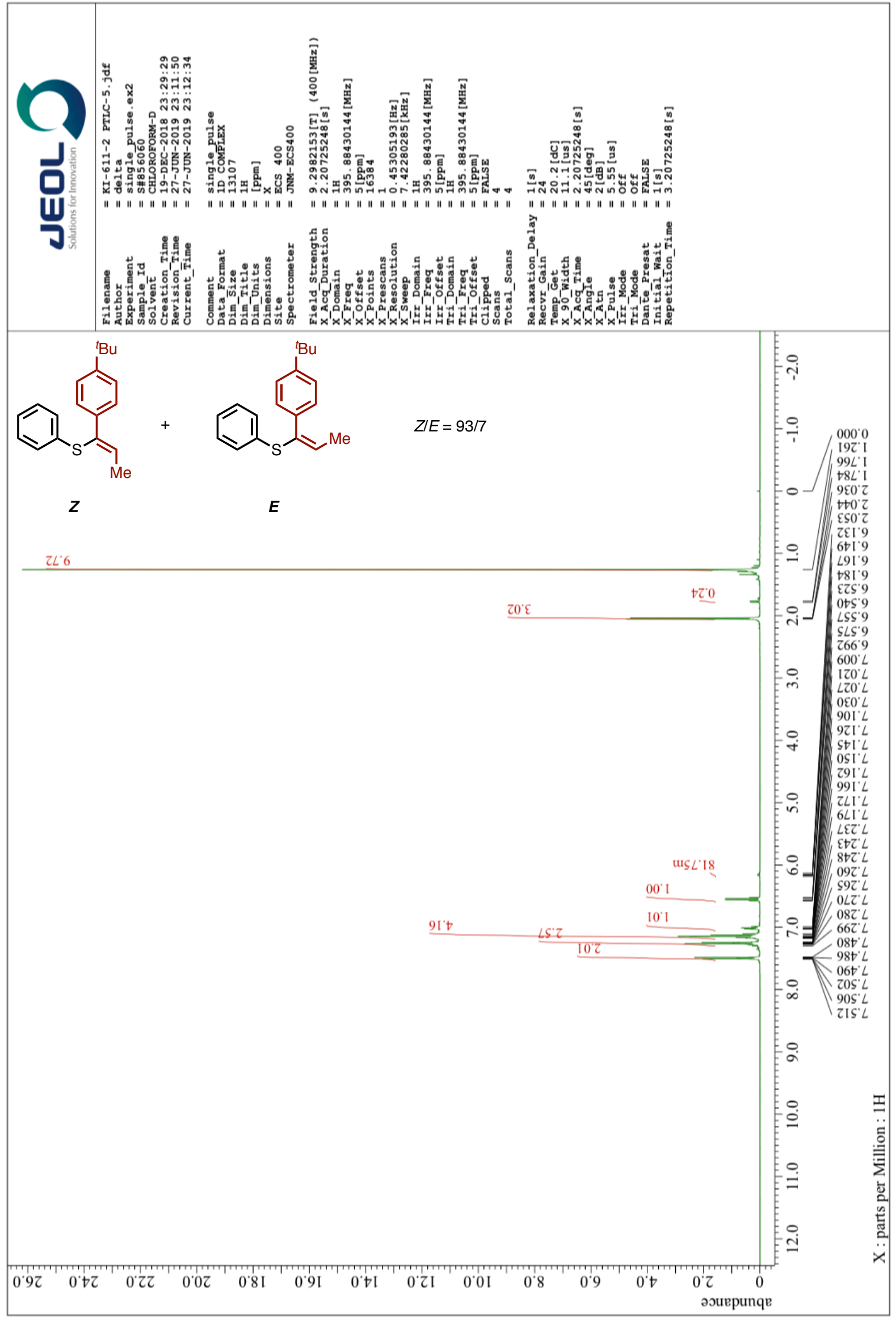


${ }^{13} \mathrm{C}$ NMR of $\mathbf{3 A j}\left(101 \mathrm{MHz}, \mathrm{CDCl}_{3}\right)$

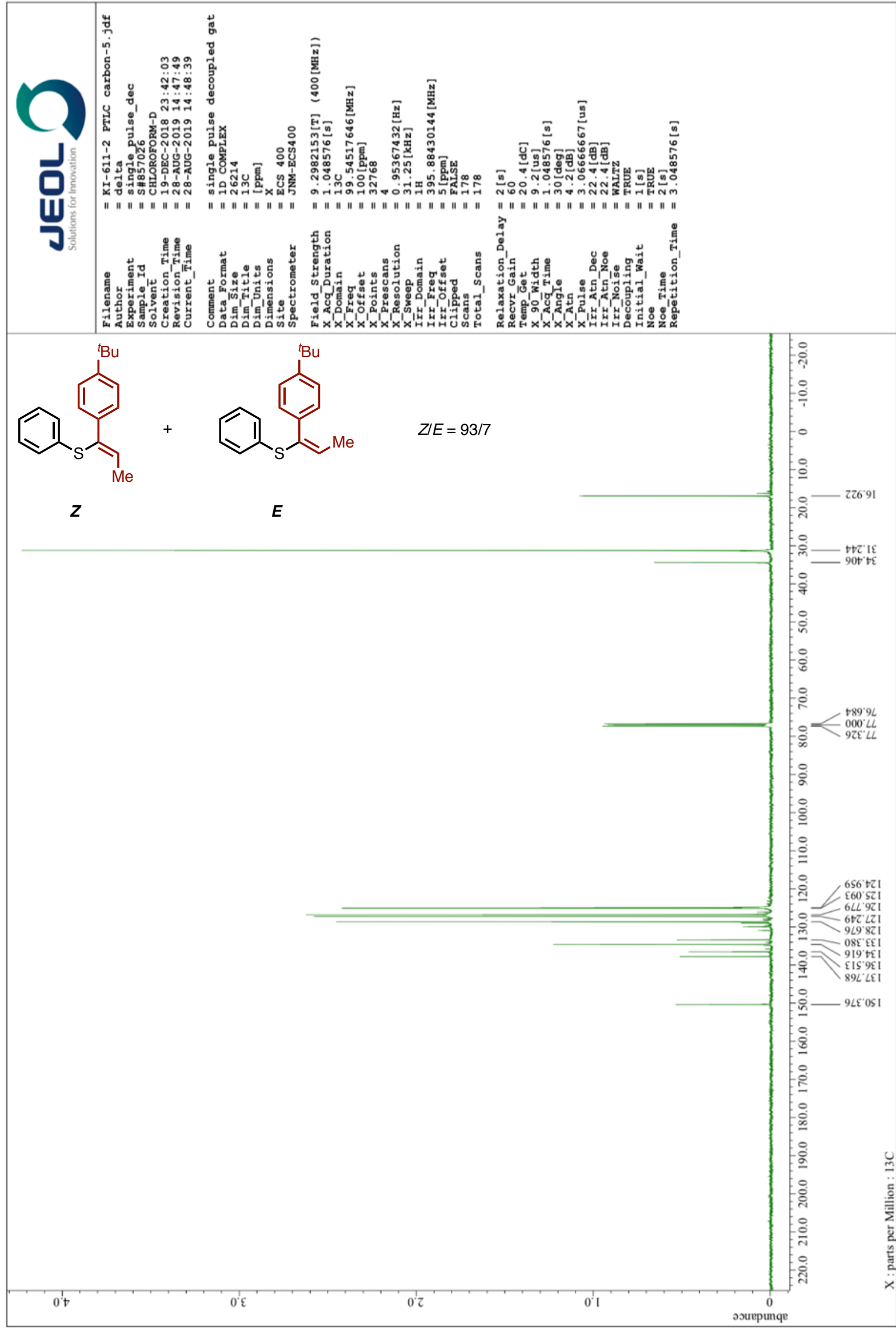


${ }^{1} \mathrm{H}$ NMR of $\mathbf{3 A k}\left(400 \mathrm{MHz}, \mathrm{CDCl}_{3}\right)$

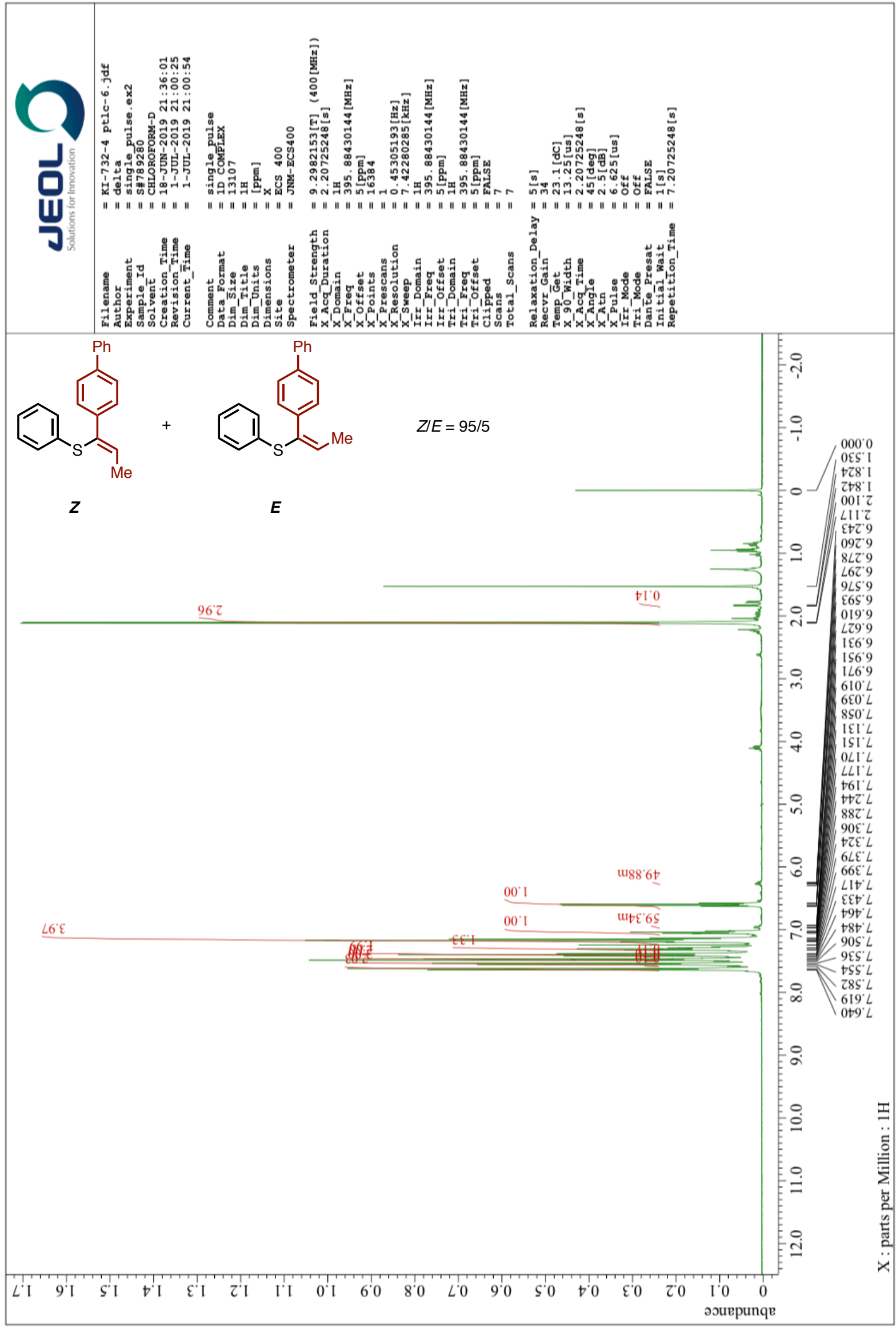


${ }^{13} \mathrm{C}$ NMR of $\mathbf{3 A k}\left(101 \mathrm{MHz}, \mathrm{CDCl}_{3}\right)$

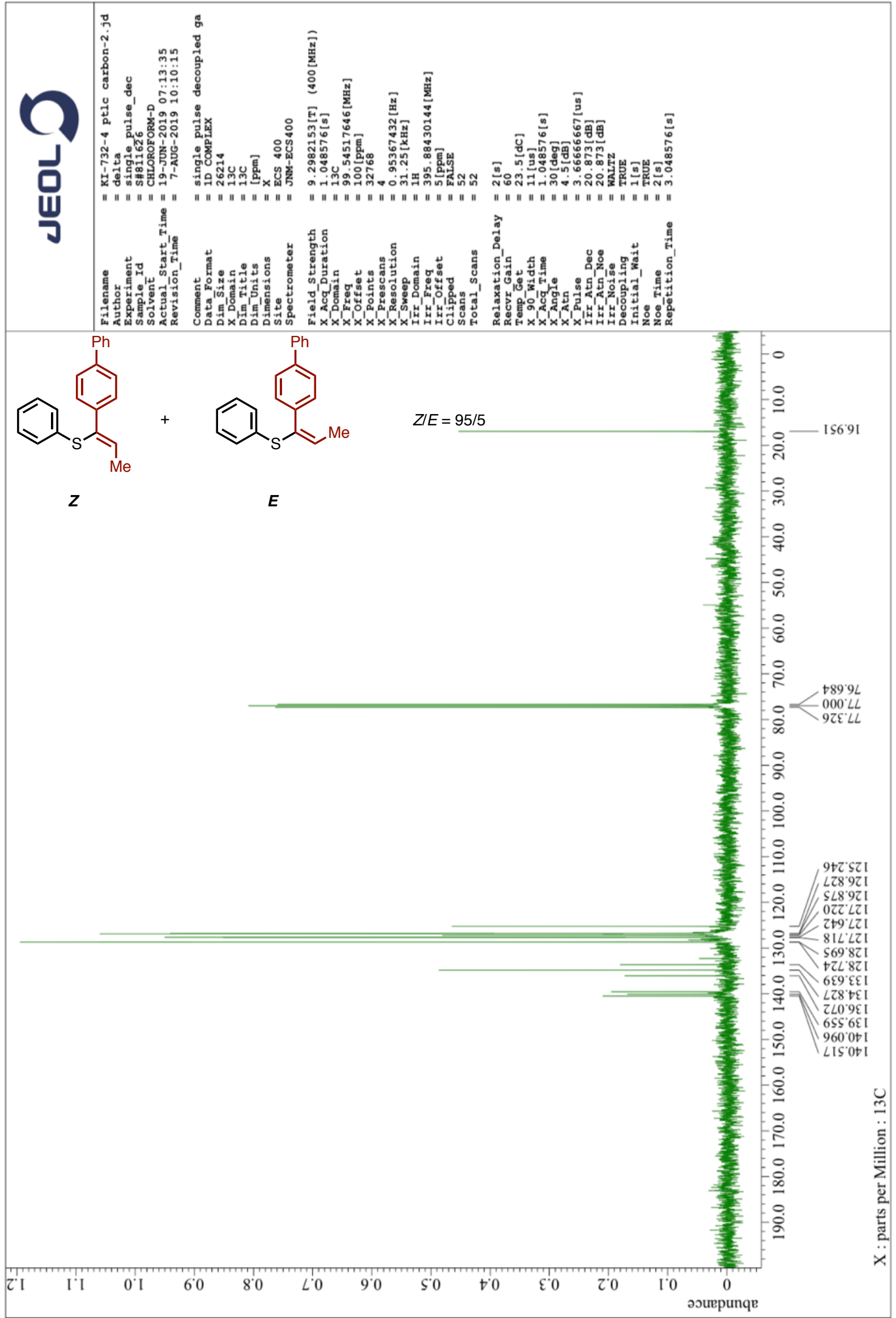


${ }^{1} \mathrm{H}$ NMR of $3 \mathrm{Al}\left(400 \mathrm{MHz}, \mathrm{CDCl}_{3}\right)$

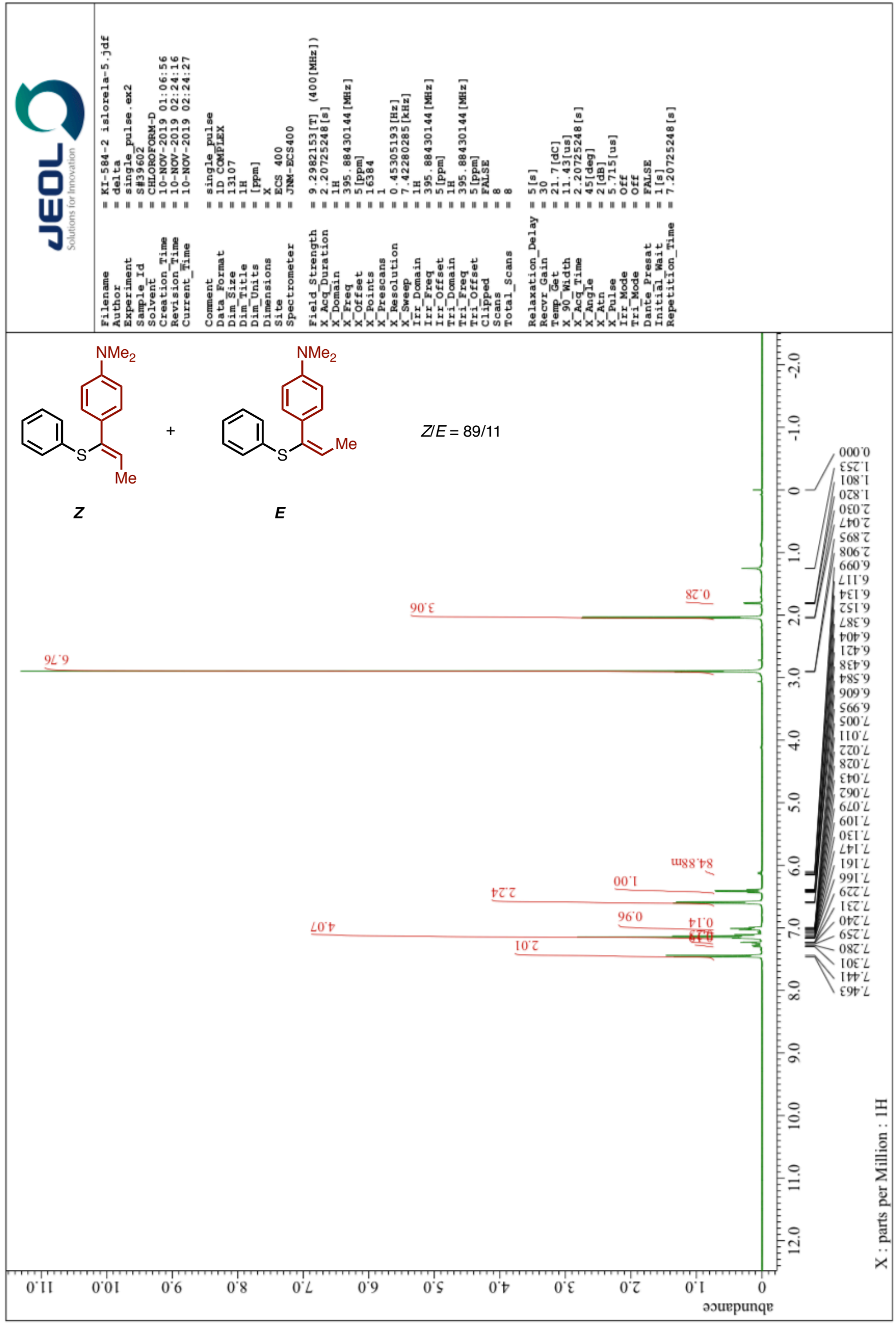


${ }^{13} \mathrm{C}$ NMR of 3Al $\left(101 \mathrm{MHz}, \mathrm{CDCl}_{3}\right)$

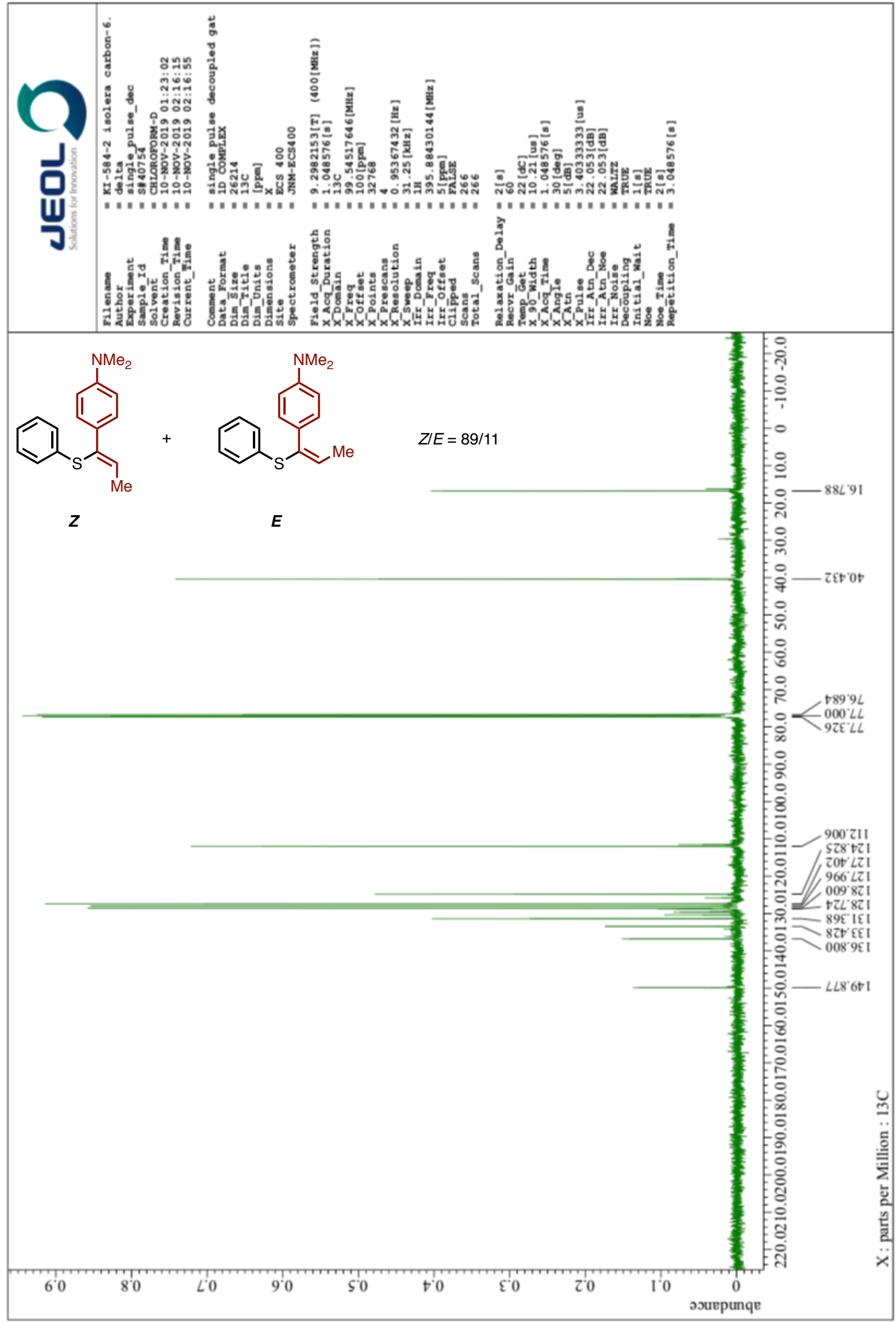


${ }^{1} \mathrm{H}$ NMR of $\mathbf{3 A m}\left(400 \mathrm{MHz}, \mathrm{CDCl}_{3}\right)$

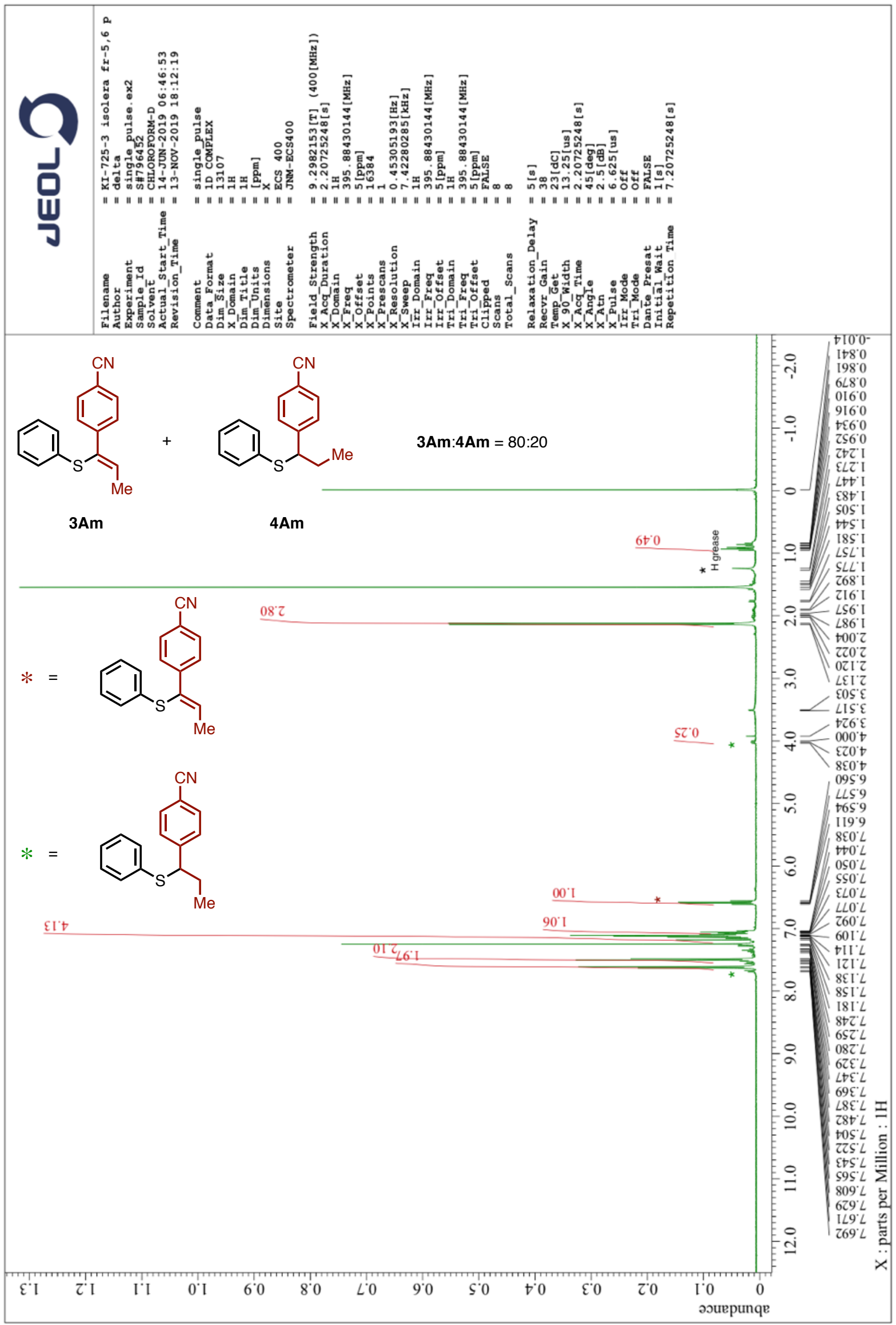


${ }^{13} \mathrm{C}$ NMR of $\mathbf{3 A m}\left(101 \mathrm{MHz}, \mathrm{CDCl}_{3}\right)$

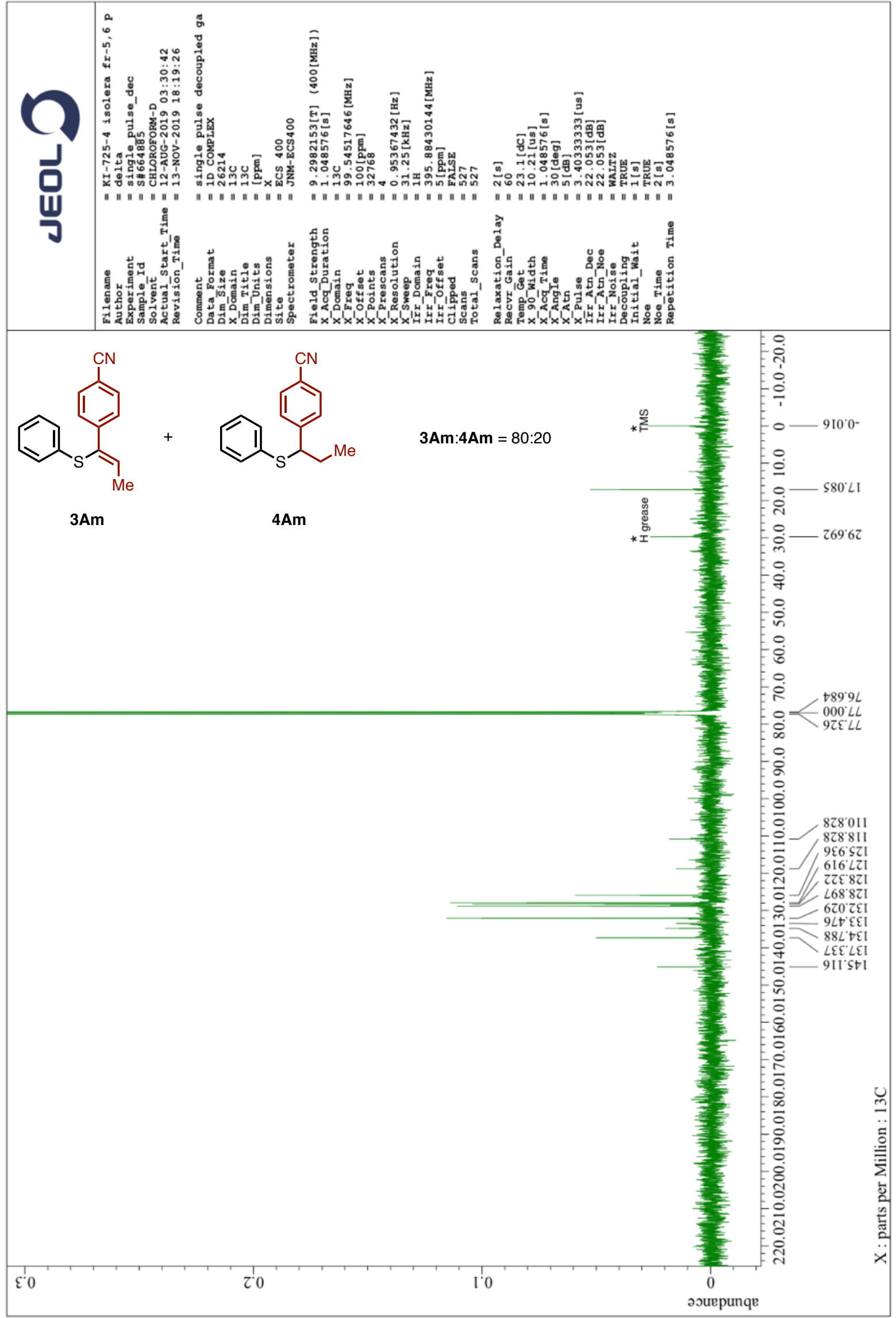


${ }^{1} \mathrm{H}$ NMR of $\mathbf{3 A n}\left(400 \mathrm{MHz}, \mathrm{CDCl}_{3}\right)$

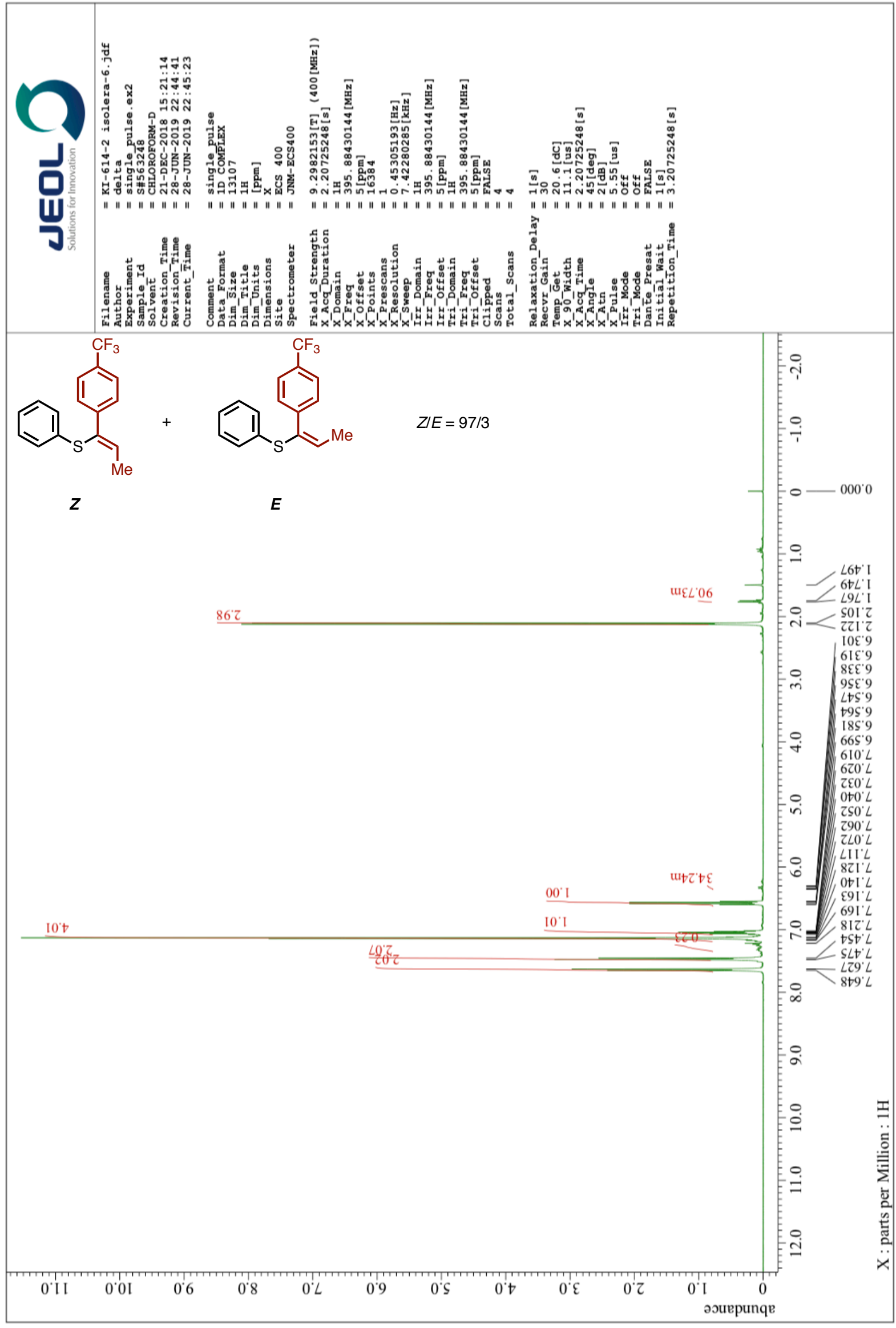


${ }^{13} \mathrm{C}$ NMR of $\mathbf{3 A n}\left(101 \mathrm{MHz}, \mathrm{CDCl}_{3}\right)$

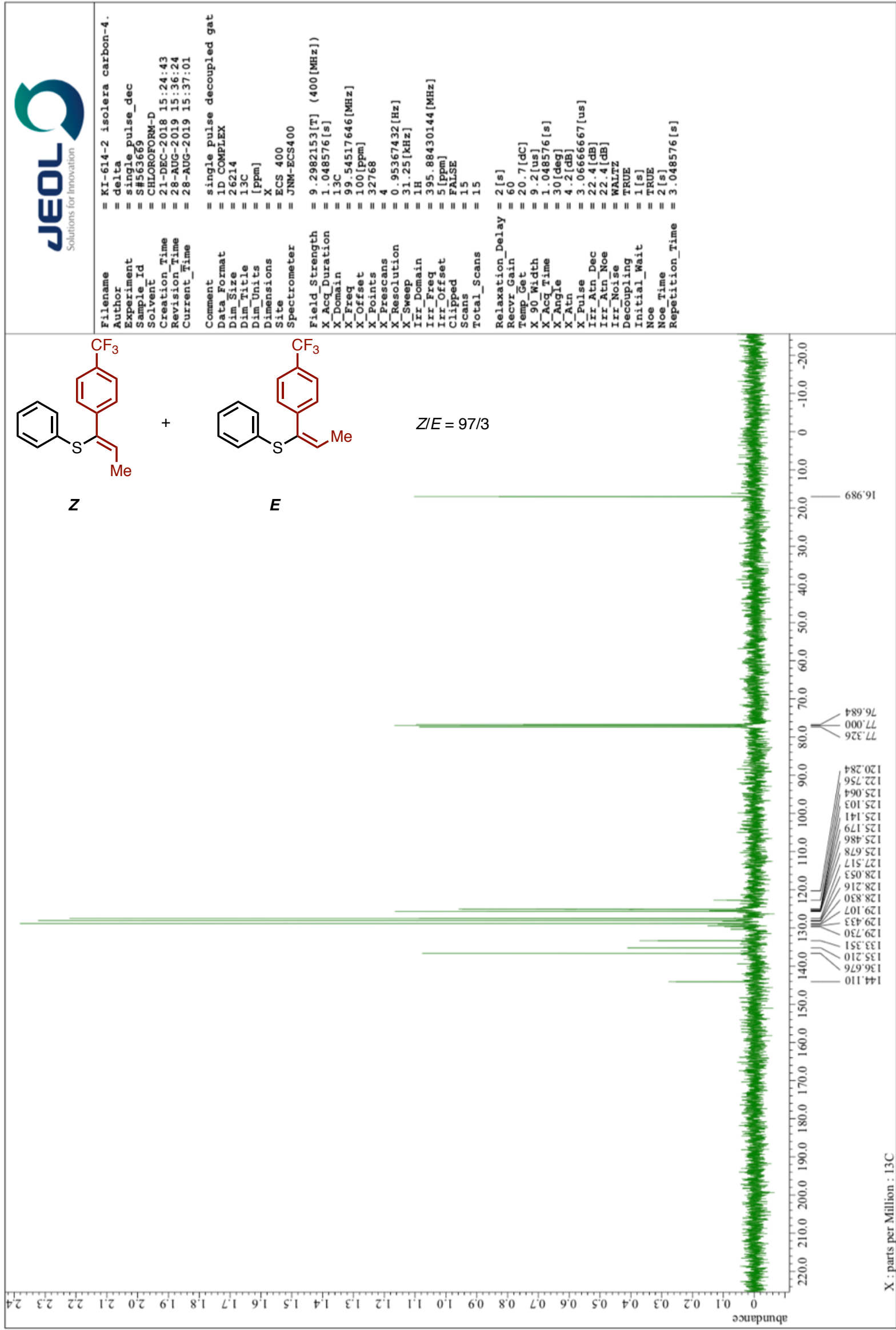


${ }^{1} \mathrm{H}$ NMR of $3 \mathrm{Ao}\left(400 \mathrm{MHz}, \mathrm{CDCl}_{3}\right)$

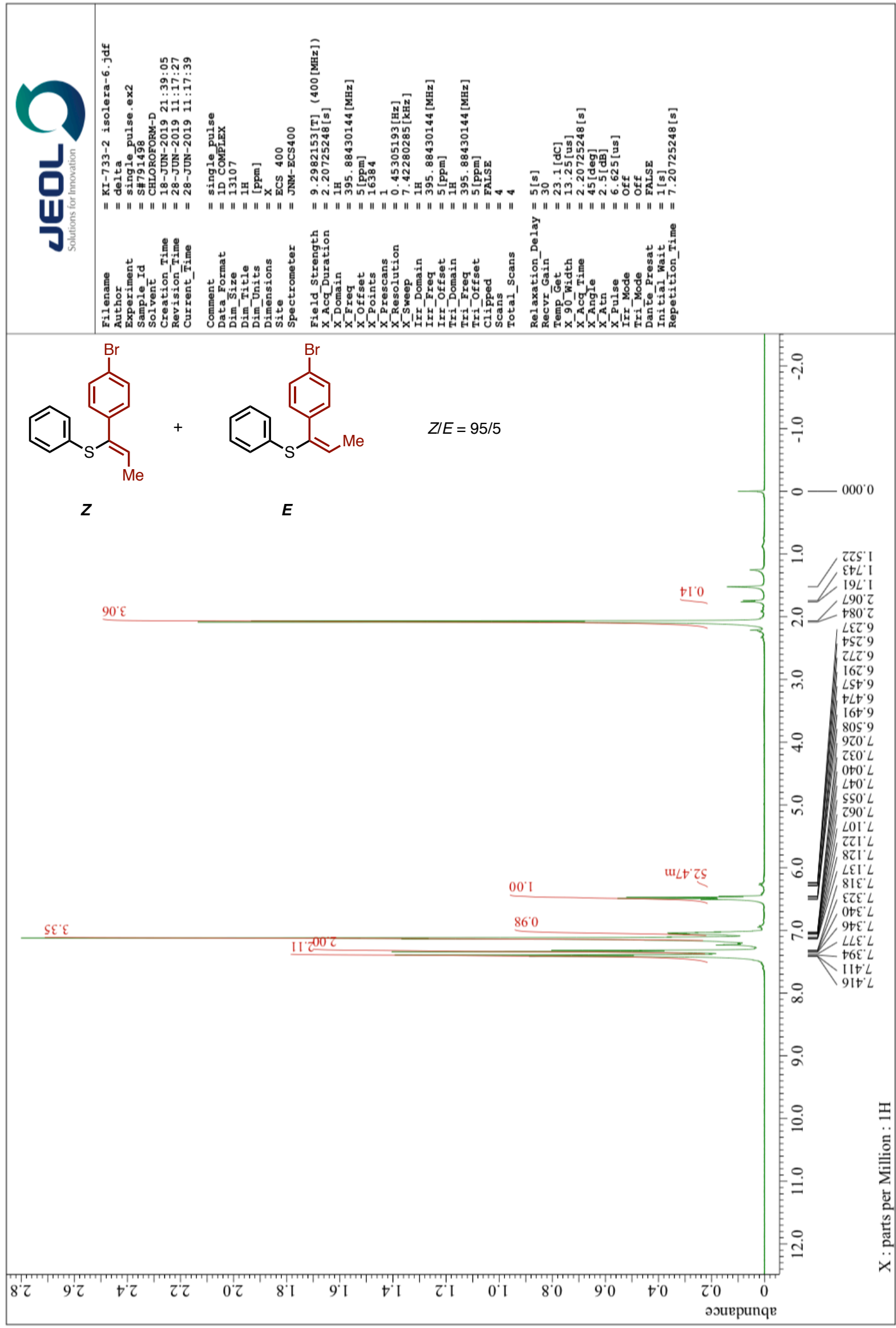


${ }^{13} \mathrm{C}$ NMR of $\mathbf{3 A o}\left(101 \mathrm{MHz}, \mathrm{CDCl}_{3}\right)$

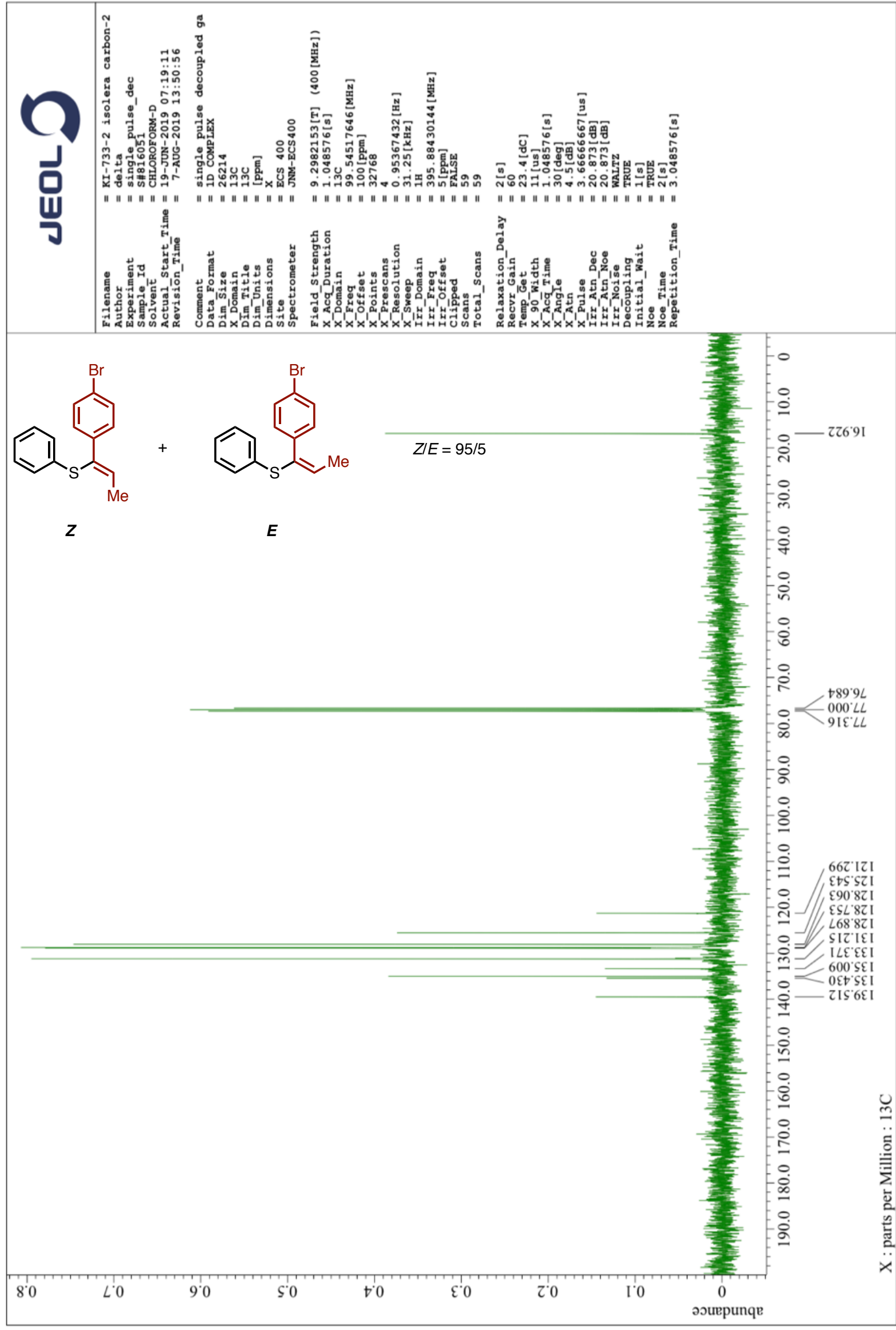


${ }^{1} \mathrm{H}$ NMR of $\mathbf{3 A p}\left(400 \mathrm{MHz}, \mathrm{CDCl}_{3}\right)$

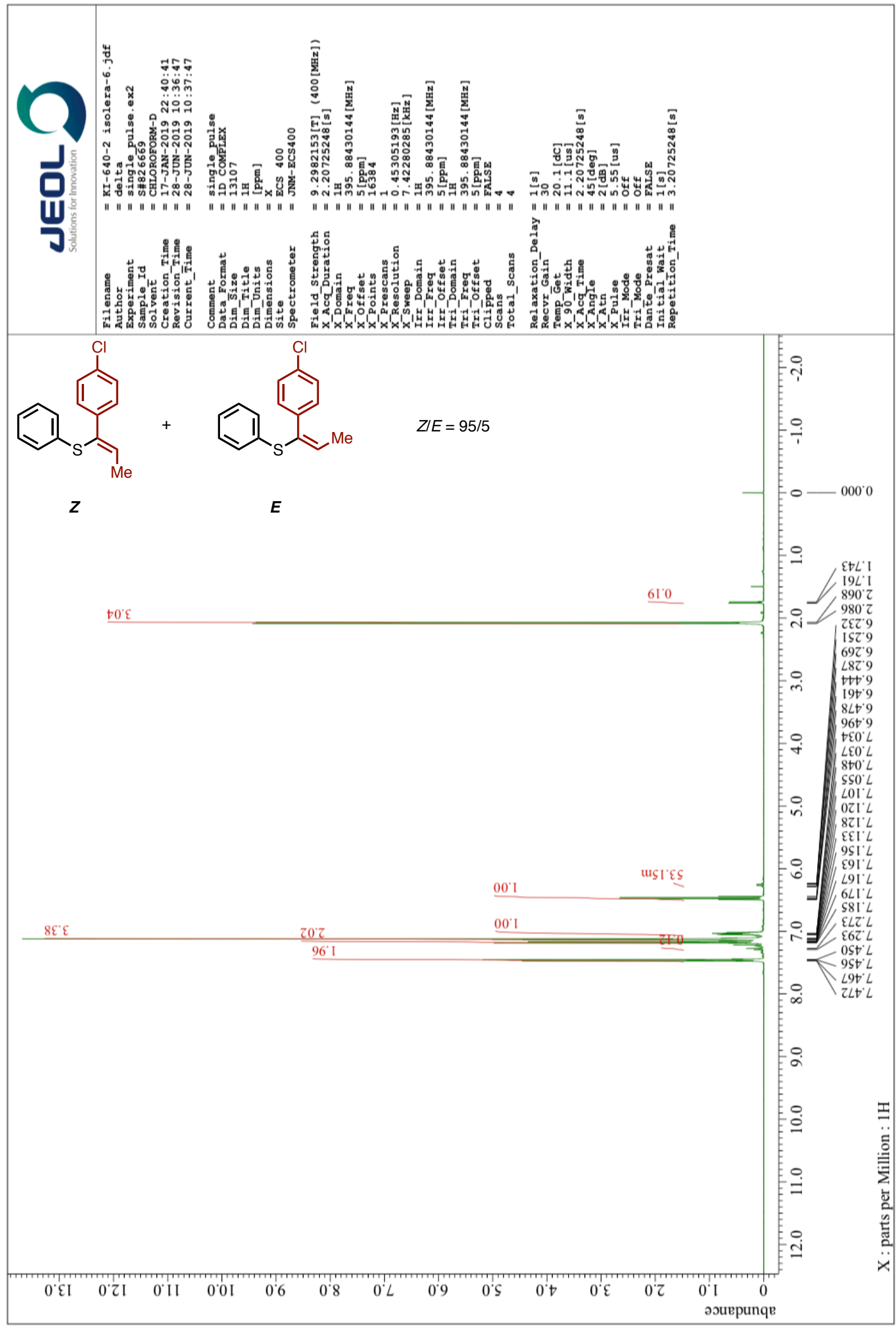


${ }^{13} \mathrm{C}$ NMR of $\mathbf{3 A p}\left(101 \mathrm{MHz}, \mathrm{CDCl}_{3}\right)$

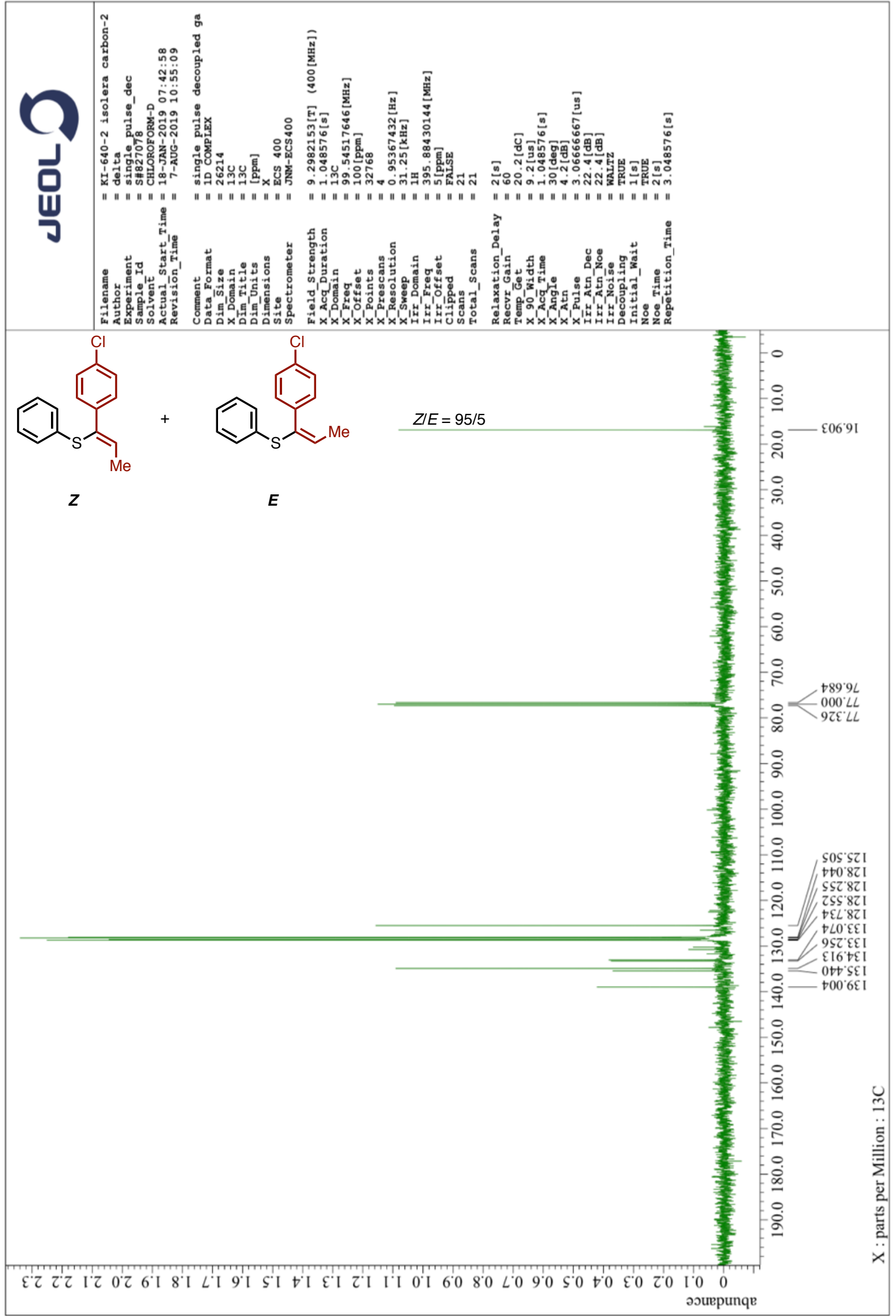


${ }^{1} \mathrm{H}$ NMR of $\mathbf{3 A q}\left(400 \mathrm{MHz}, \mathrm{CDCl}_{3}\right.$ )

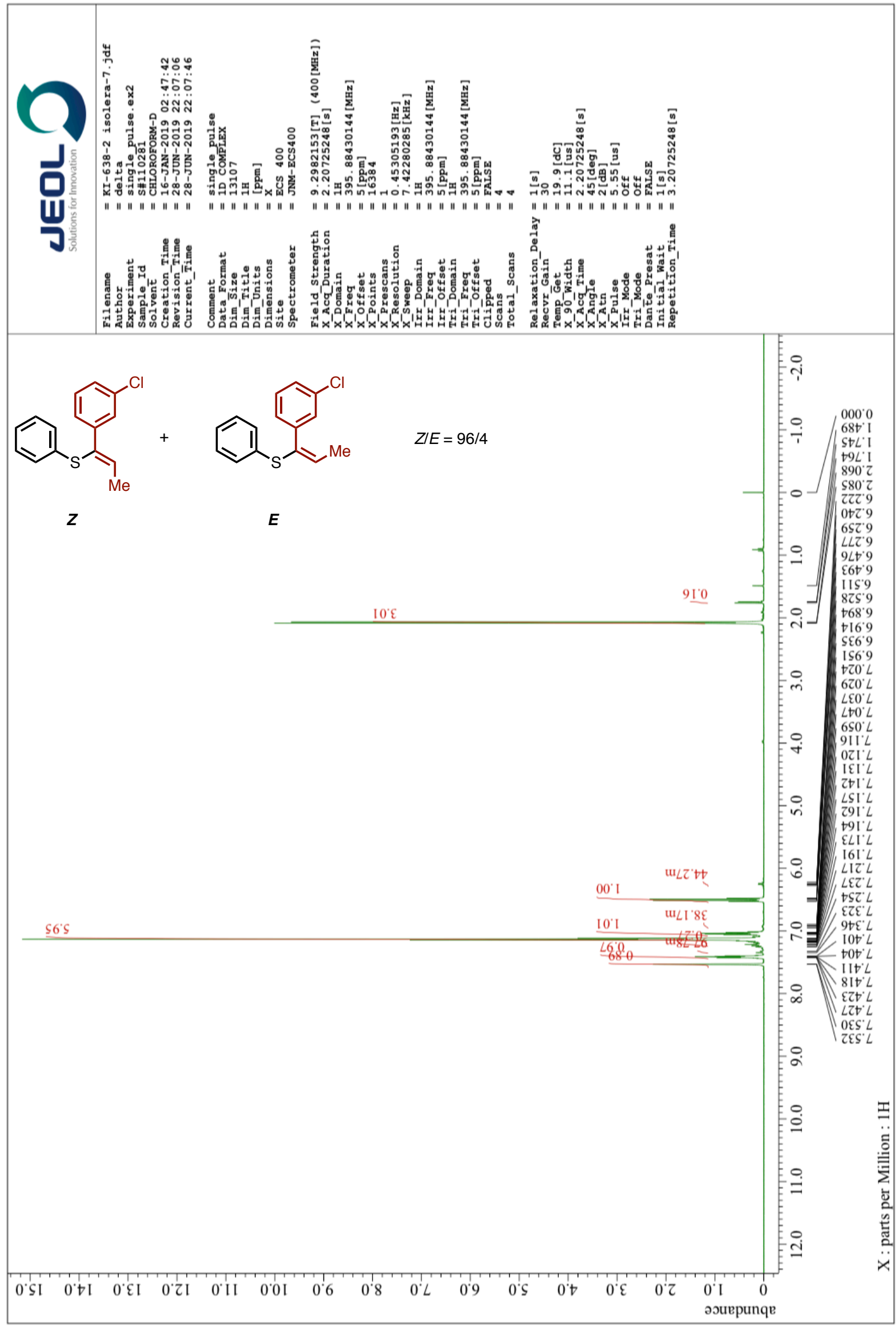


${ }^{13} \mathrm{C}$ NMR of $\mathbf{3 A q}\left(101 \mathrm{MHz}, \mathrm{CDCl}_{3}\right)$

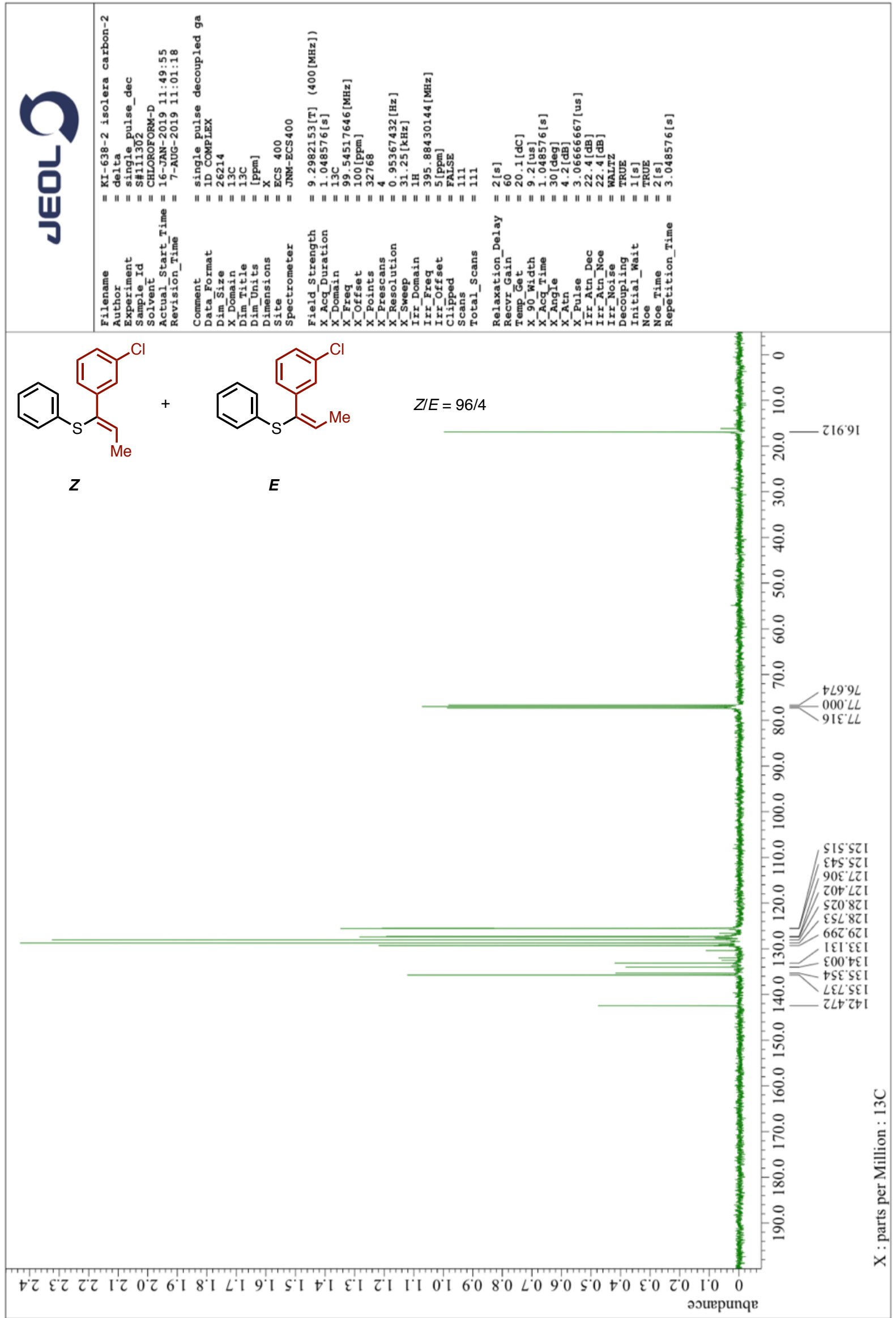


Supporting Information (Ishitobi, Muto, Yamaguchi) Pd-Catalyzed Alkenyl Thioether Synthesis from Thioesters and N-Tosylhydrazones

${ }^{1} \mathrm{H}$ NMR of $\mathbf{3 A r}\left(400 \mathrm{MHz}, \mathrm{CDCl}_{3}\right)$

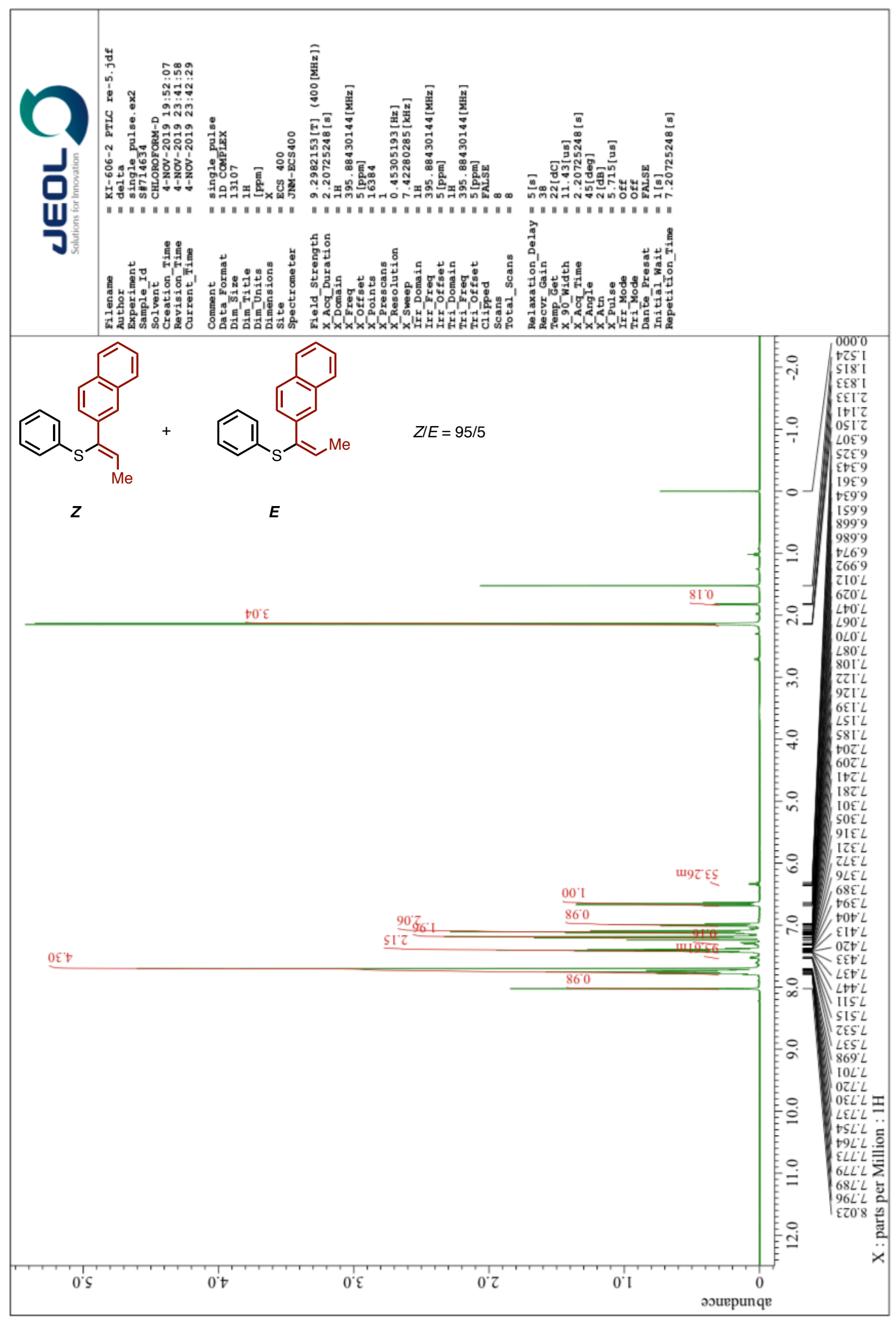

S141 
${ }^{13} \mathrm{C}$ NMR of $\mathbf{3 A r}\left(101 \mathrm{MHz}, \mathrm{CDCl}_{3}\right)$

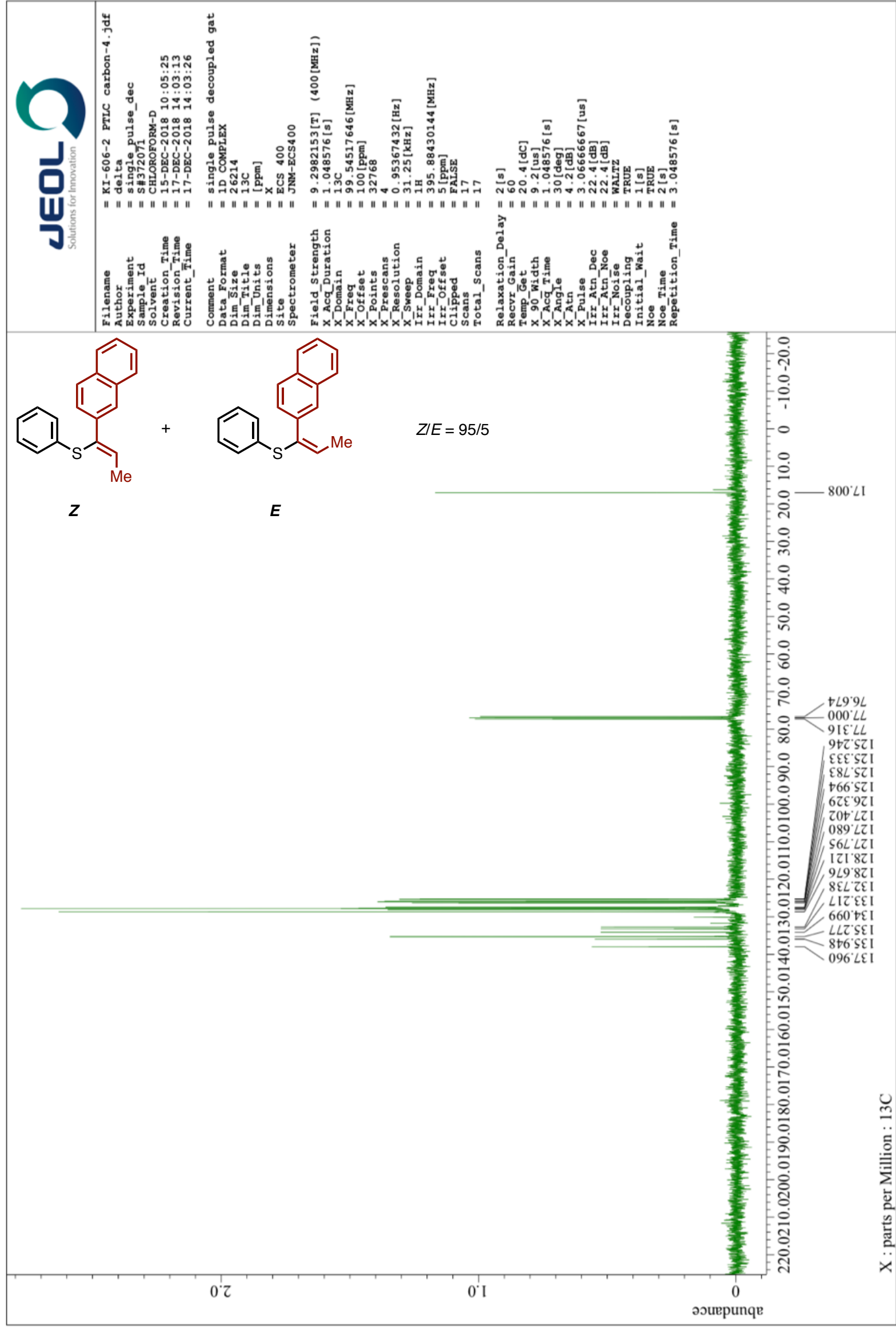


${ }^{1} \mathrm{H} \mathrm{NMR}$ of $\mathbf{3 A s}\left(400 \mathrm{MHz}, \mathrm{CDCl}_{3}\right)$

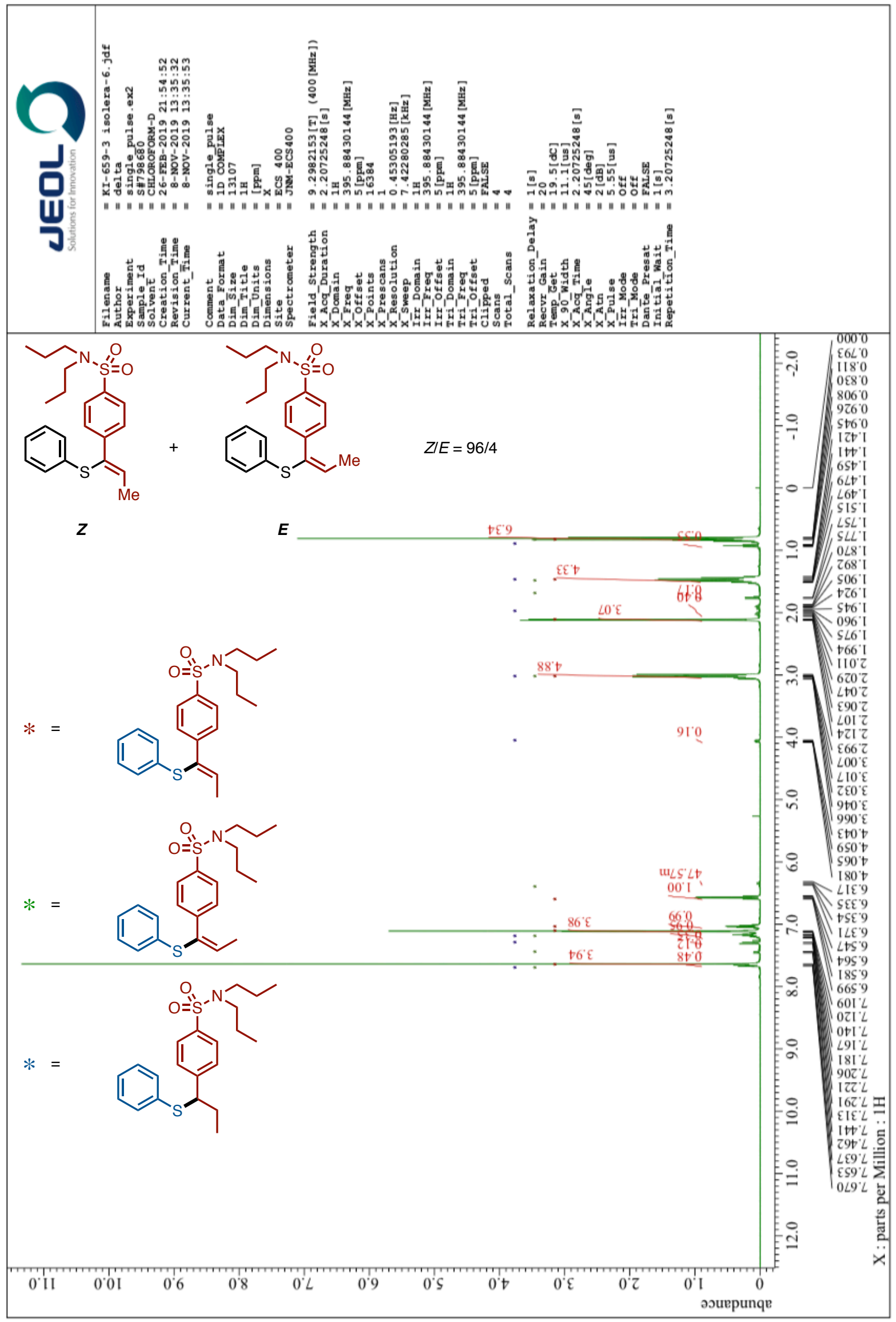


Supporting Information (Ishitobi, Muto, Yamaguchi) Pd-Catalyzed Alkenyl Thioether Synthesis from Thioesters and $\mathrm{N}$-Tosylhydrazones

${ }^{13} \mathrm{C}$ NMR of $\mathbf{3 A s}\left(101 \mathrm{MHz}, \mathrm{CDCl}_{3}\right)$

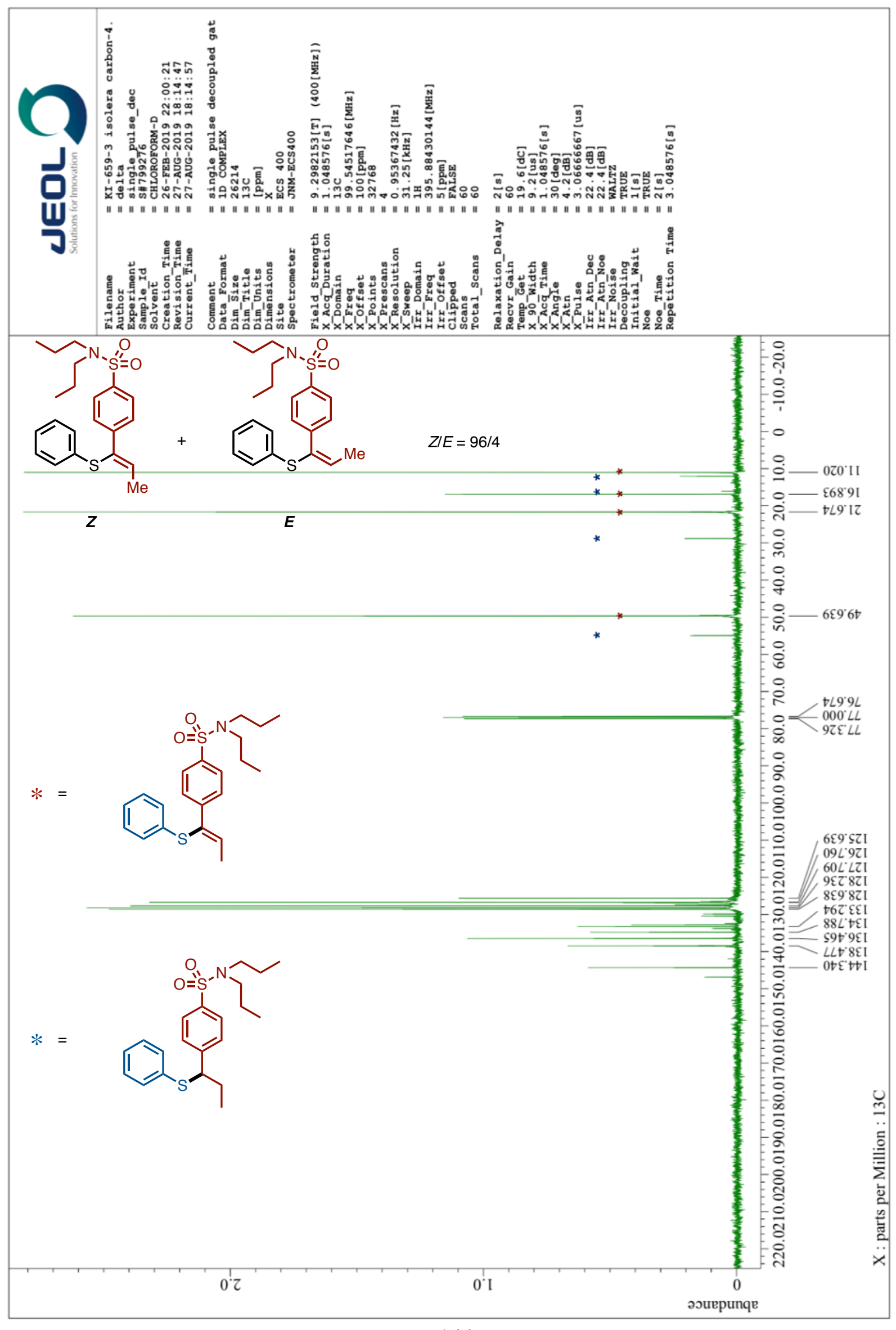

S144 
${ }^{1} \mathrm{H}$ NMR of $5\left(400 \mathrm{MHz}, \mathrm{CDCl}_{3}\right)$

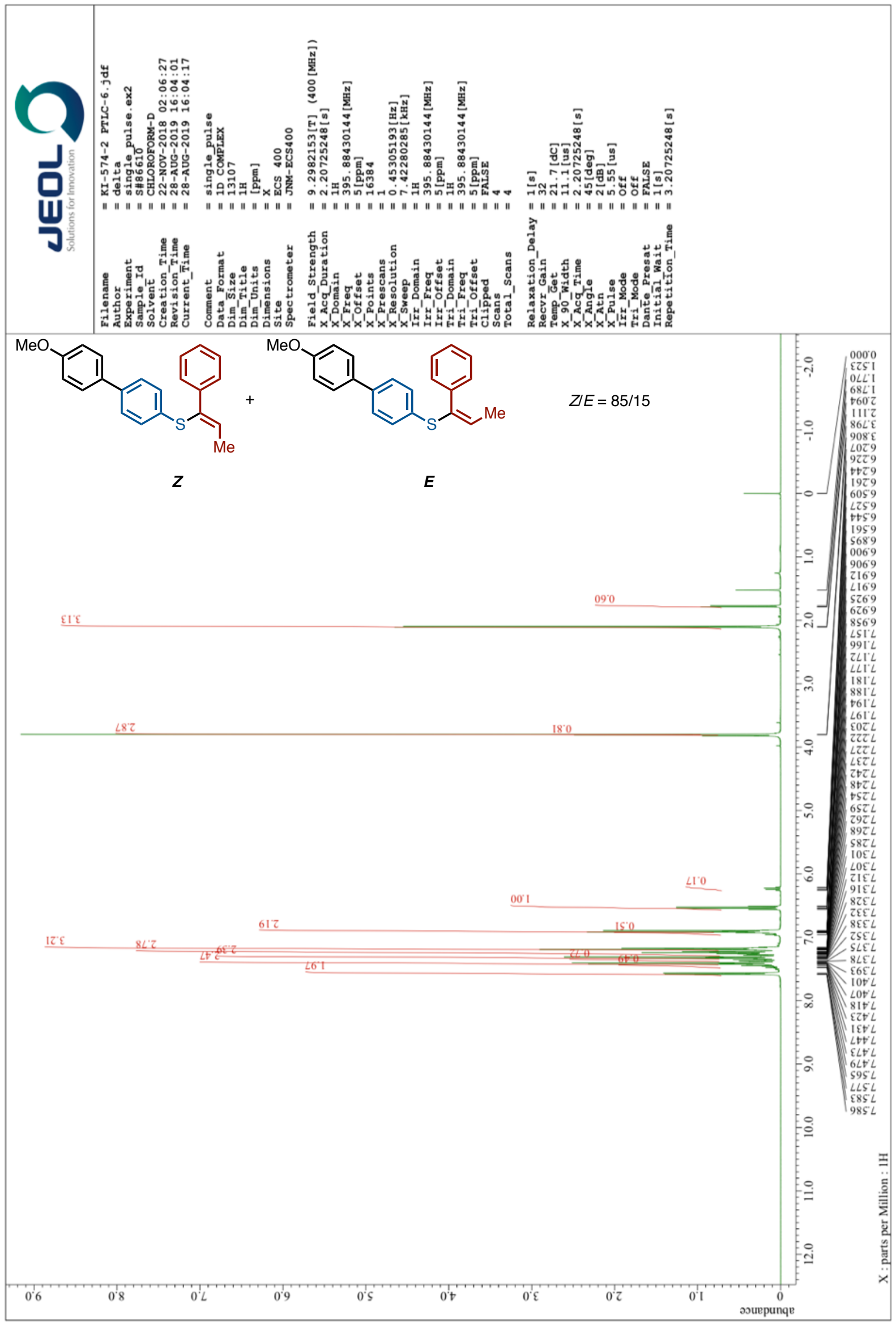


${ }^{13} \mathrm{C}$ NMR of $5\left(101 \mathrm{MHz}, \mathrm{CDCl}_{3}\right)$

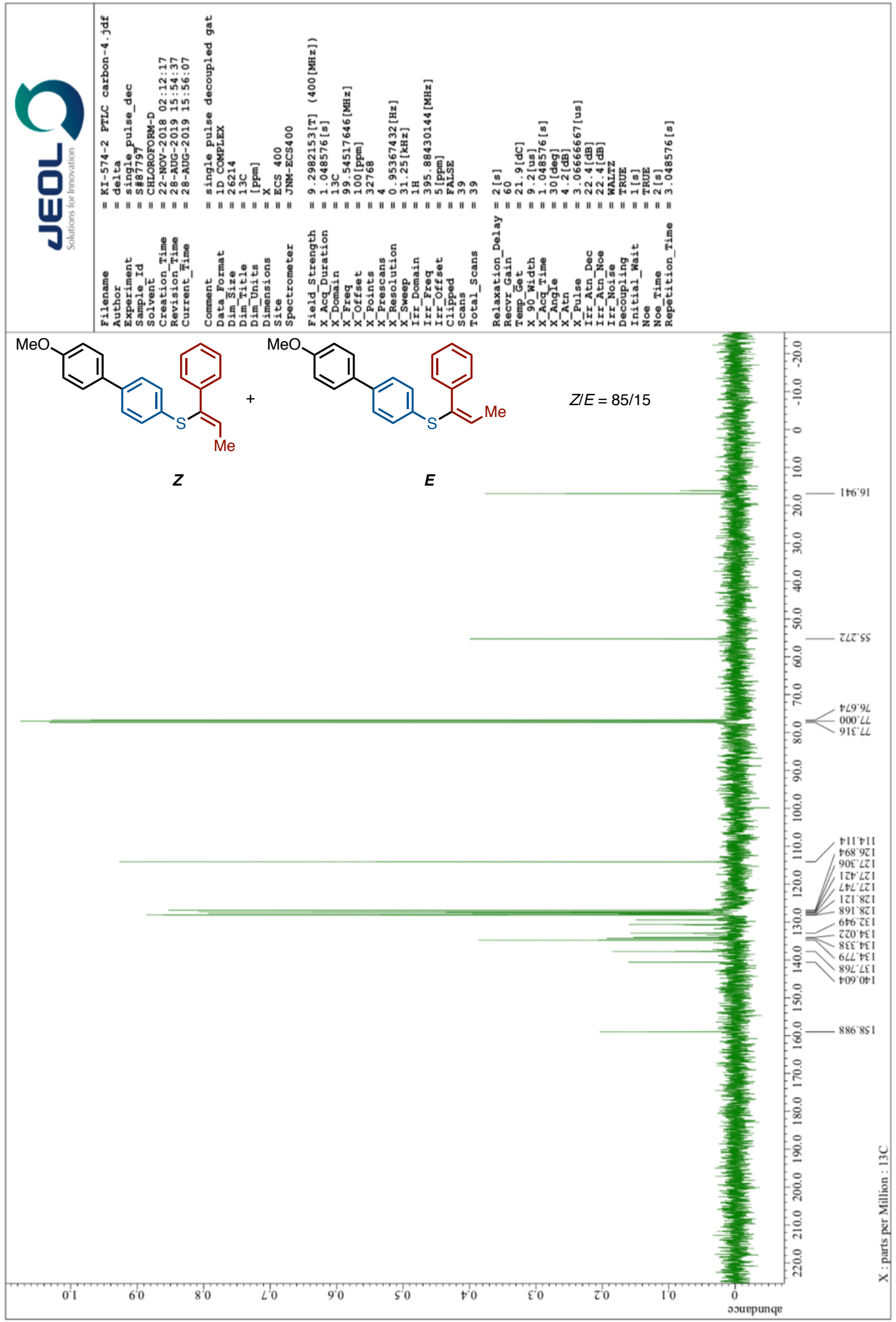


${ }^{1} \mathrm{H}$ NMR of $7\left(400 \mathrm{MHz}, \mathrm{CDCl}_{3}\right)$

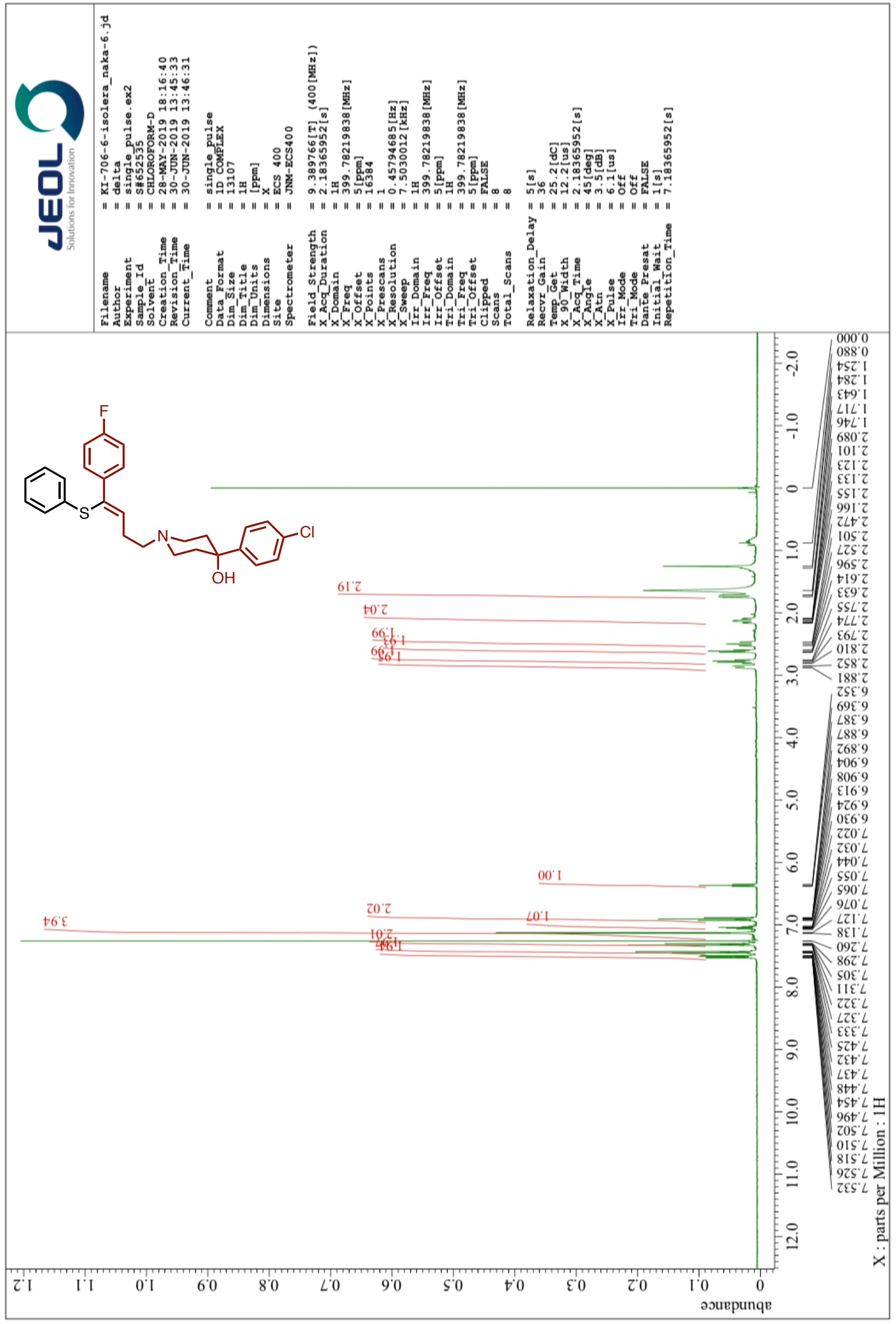


${ }^{13} \mathrm{C}$ NMR of $7\left(101 \mathrm{MHz}, \mathrm{CDCl}_{3}\right)$

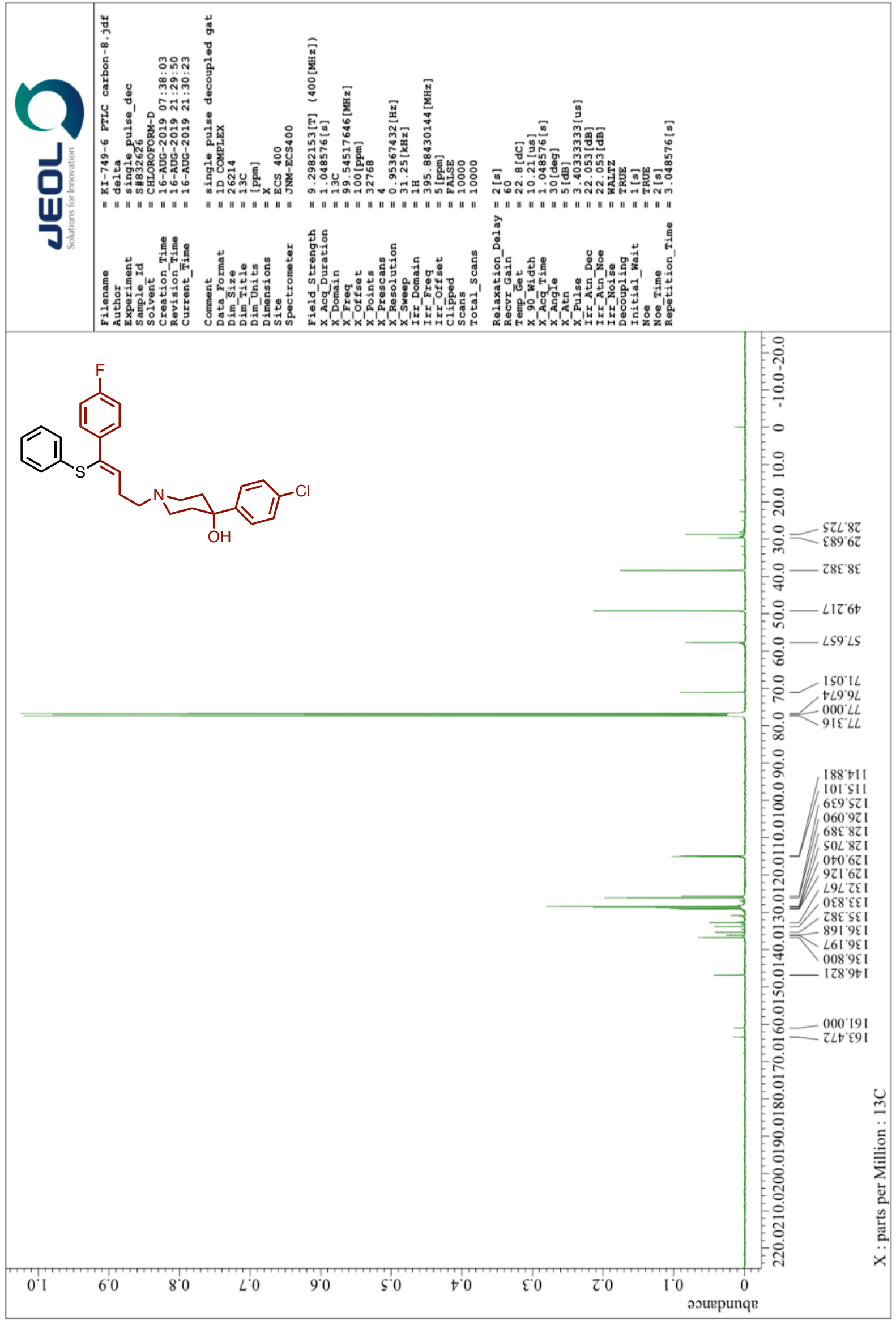

